


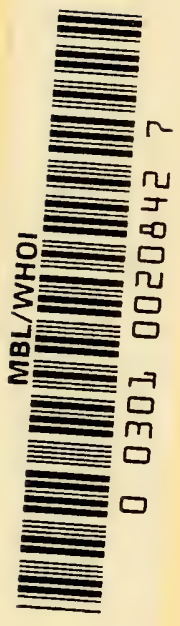



A SYMPOSIUM ON RESPIRATORY ENZYMES 


\section{A Symposium on \\ RESPIRATORY ENZYMES}

Contributors:

Otto Meyerhof

Eric G. Ball

Fritz LipManN

Kurt G. Stern

Fritz Schlenk

T. R. Hogness

Elmer Stotz

Carl F. Cori

E. A. Evans, JR.

Philip P. Cohen

K. A. C. Elliott

Dean Burk

C. J. Kensler
Erwin HaAs

H. M. KALCKAR

M. J. JOHNSON

VAN R. PotTer

H. G. WOOD

R. H. Burris

C. H. Werkman

P. W. Wilson

F. F. NORD

Ephraim Shorr

A. E. Axelrod

Frederick Bernheim

E. S. G. Barron

Fredrick J. Stare

The University of Wisconsin Press MADISON 


\section{Copyright 1942 BY THE UNIVERSITY OF WISCONSIN \\ All Rights Reserved}

PRINTED IN THE UNITED STATES OF AMERICA 


\section{Foreword}

$T^{\mathrm{N}} 1897$ Buchner published his classical study on alcoholic fermenI tation by cell-free yeast juice. In the same year Eijkman concluded that beri-beri among the natives of the Dutch East Indies was caused by a dietary deficiency arising from the use of polished rice. These two discoveries may be said to have initiated the modern investigations in two of the most important fields of biochemistry and medicine: the nature of the respiratory enzymes and the function of the vitamins in cellular metabolism.

For the next thirty years research workers in these two fields pursued their investigation almost independently of one another and more or less oblivious to the progress being made in the other's field. Then in the early part of the last decade it was discovered that riboflavin was the functional group in a respiratory enzyme and very soon afterward this compound was shown to be vitamin $B_{2}(G)$. Further discoveries of a similar nature soon demonstrated that the enzyme chemist and the nutritionist were to a great extent prospecting the same territory. The time seemed to be ripe, therefore, for the two groups to join in a discussion of the latest advances. Such a meeting on the "Respiratory Enzymes and the Biological Action of the Vitamins" was sponsored jointly by the Universities of Wisconsin and Chicago, institutions that have long been leaders in these fields.

This book contains the lectures and discussions given at the University of Wisconsin. It deals with the fundamental nature of those enzymes that are intimately connected with the functioning of the vitamins. Informative presentation of the latest developments, interpretation of past and present findings, and indication of some of the problems still unsolved in respiratory enzyme research are given by recognized international authorities in the field. Supplementing these explanations of the fundamental nature of respiratory enzymes are discussions applying the findings to specific problems.

The Program Committee wishes to thank the many members of the faculty for their cooperation in arranging the meetings held at the University of Wisconsin, the speakers for their papers and discussions, and the Wisconsin Alumni Research Foundation for the grant which made these sessions and the publication of the present volume possible. 



\title{
Address of Welcome
}

\author{
C. A. DYKSTRA \\ President of The University of Wisconsin
}

to University of Wisconsin is a happy host today. It welcomes
to its campus scientists from many laboratories who are drawn together for the discussion of common problems and common aims. It recognizes in this symposium the challenge that faces intelligent men of good will everywhere-the great need there is in the contemporary world for sitting down and reasoning together. From such a process comes progress.

We are concerned here with functions which operate in the biological and chemical world. We seek these out and discover how they work so that, knowing about them, we may cooperate with nature for the good of man. This we do by observation, experimentation, analysis, and, finally, the objective setting down of results that may yield a pattern or a principle. As we look about us and see biological specimens called men reacting to special or group interests as passion and selfishness may happen to dictate, we ask ourselves, a bit dismally perhaps, whether the statesmen and public leaders of the world can ever be persuaded to try out the scientific method as an approach to the problems of world organization. We also need desperately a healthy society and a sound international body.

Here, today, we pay tribute to the internationalism of science. As we scan our program for the week we are struck by the fact that men from different backgrounds and from many nationalities and races can come together peacefully in a symposium to present the results of long years of human effort in a field of science, check these results, and try to establish what they mean or may mean to life on this planet. Today and right here men labor together who, were they still living in their family homelands, would be enemies, legally and politically. This is the great modern paradox-that as the world of communication has made the globe a unity and as the domains of science, literature, music, art, commerce, and industry have become international, we have at the same time the phenomenon of a more bitter nationalism than ever before. Something is wrong that needs 
early correction, and intelligent men must give attention to the challenge.

We meet today to talk of many things in the wonderland of science. We have the special vocabulary necessary for the accuracy of our thinking and investigation. This vocabulary is a closed book to the man in the street except for a few words, such as vitamin, for instance. This man in the street, however, does get a partial implication of your work as he hears or reads the advertiser who expounds the merits of certain food products. He may even be led to think that he can be a vigorous and whole man if only he has a box of pills or capsules in his vest pocket. He may even be duped or exploited because of this partial knowledge.

We therefore have the obligation in our special fields of science which promise so much to all to attempt such simplification and general statement that those things for which we can vouch will become common knowledge at the earliest possible moment. Just now there is a wide spread of interest in many areas which this symposium deals with. It is a good time, therefore, to capitalize on this popular interest, for we have a receptive public. We of the public are willing and anxious to learn from you.

We here at Wisconsin are glad you are with us. We are happy too in the cooperation of our sister institution, the University of Chicago, in the enterprise here represented. Our welcome is genuine, and we wish for the conference unusual and distinguished success. 


\section{Contents}

\section{INTERMEDIATE CARBOHYDRATE METABOLISM}

Introduction, 3

Inadequacy of older views of the relation between aerobic and anaerobic carbohydrate breakdown, 4 Alternate pathways of oxidation, 4 The oxidation quotient, 5

Competition of oxygen and pyruvate in oxidation of dihydrocozymase, 6 Aerobic phosphorylation, 7

Reversibility of the glycolysis reactions, 9

Formation of phosphopyruvate from pyruvate through oxalacetate, 12

Inhibitors, 14

\section{OXIDATIVE MECHANISMS IN ANIMAL TISSUES}

By Eric G. Ball, Harvard Medical School . . . . . . . 16

Introduction, 16

Oxygen activation and the cytochrome system, 17

Substrate activation and the pyridine nucleotide and flavoprotein systems, 18

The energy relationships of these systems and the transfer of electrons between them, 21

The relative concentrations of cytochrome $c$, diphosphopyridine nucleotide, and flavoprotein in animal tissues and their possible significance, 25

The possibility of the existence of pathways alternate to the cytochrome system, 26

Cyanide poisoning of respiration and a theory of its mechanism, 27

An interpretation of respiratory mechanisms in the arbacia egg, 30 Azide poisoning of respiration and a possible interpretation of its action on muscle respiration, 30

\section{DISCUSSION ON HYDROGEN TRANSPORT}

\section{Van R. Potter, chairman . . . . . . .}

The Possible Role of Intermediany Metabolites as Hydrogen Carriers by K. A. C. Elliott, 33

The Role of the Carriers in Dismutations and Coupled OxidoReductions, WITH Special ReferENCE TO THE Flavoproteins by
E. G. Ball and F. Lipmann, 38

The Physico-Chemical Mechanism of Hydrogen Transport by Kurt Stern and Erwin Haas, 42

Possibility of a By-Pass aRound The Cytochrome System by Elmer Stotz, 46

\section{PASTEUR EFFECT}

By Fritz Lipmann, Massachusetts General Hospital .

The efficiency of aerobic and anaerobic metabolism, 50

The metabolic structure of cells, 52 Bacteria, 53

Animal tissues, 56

Interpretation of the Pasteur effect, 59

Equilibrium schemes, 59
Inhibition of the Pasteur effect, 62 Reversible oxidative inhibition of glycolysis in extracts, 65 Thiol influence on fermentation and glycolysis in intact cells, 67 Pasteur effect with very low respiration, 69

Conclusion and outlook, 70 
OXIDASES, PEROXIDASES, AND CATALASE

By Kurt G. Stern, Yale University School of Medicine . . . . 74

Introduction, 74

The common denominator in hemin catalyses, 74

Enzyme-substrate intermediates, 84
On the mechanics of hemin catalyses, 86

Autoxidizable iron compounds, 96

Oxygen transfer in living cells, 99

\section{NICOTINAMIDE NUCLEOTIDE ENZYMES}

By Fritz Schlenk, School of Medicine, University of Texas .

Historical introduction, 104

Codehydrogenase I and II, 104

Occurrence, 105

Preparation, 106

Properties, 108

Investigation of the structure of codehydrogenase I and II, 109

Reversible reduction, 109

The "model compounds" of Karrer, 111

Nicotinamide nucleoside, its preparation and properties, 113
Structure of codehydrogenase I and II, 116

Methods of determination, 120

Apodehydrogenases dependent on nicotinamide nucleotides, 122

Substrate and coenzyme specificity of apodehydrogenases, 123

Mode of action of nicotinamide nucleotide enzymes, 125

Spectrophotometric methods, 126

Biosynthesis of the codehydrogenases

I and II, 126

\section{THE FLAVOPROTEINS}

By T. R. Hogness, University of Chicago . . . . . . . 134

Historical introduction, 134

The general properties of the known yellow enzymes, 137

Dissociation constants, 139
Comparison of activities, 142

Cytochrome $c$ reductase: test, spectroscopic demonstration, and properties, 144

\section{CYTOCHROMES}

By Elmer Stotz, Harvard University .

Properties of the cytochrome components, 149

Cytochrome $c$ : isolation, purification, structure, and oxidationreduction potential, 149

Cytochrome $b, 153$

Cytochrome $a, 154$

Oxidation-reduction potential of the cytochromes in yeast, 154

Cytochrome oxidase and cytochrome $a_{3}, 155$

Identity with Warburg's enzyme, 157

Summary of absorption spectra of the cytochromes, 158

Copper-containing oxidase, 159

Soluble cytochrome $c$ peroxidase, 160
Physiological reduction of the cytochromes, 161

By the succinate system-extra factor, 161

By flavoprotein (cytochrome reductase), 163

Catalytic relations of the cytochromes and oxidase: oxidation of hydroquinone and $p$-phenylene diamine, 164

Determination and distribution of cytochrome $c$ and cytochrome oxidase, 166

Physiological functioning of the cytochrome system, 168

Possibility of a by-pass of the cytochrome system, 169 


\section{PHOSPHORYLATION OF CARBOHYDRATES}

By Carl F. Cori, Washington University School of Medicine, St. Louis 175

Introduction, 175

Uptake of inorganic phosphate, 175 Intramolecular migration of phosphate, 179
Transphosphorylation, 181

Regeneration of inorganic phosphate, 185

Summary, 188

\section{DISCUSSION ON PHOSPHORYLATION}

H. M. Kalckar, chairman

Myokinase; dephosphorylation

By H. M. Kalckar, 190

Dephosphorylating and transphosphorylating enzymes

By Otto Meyerhof, 192

\section{METABOLIC CYCLES AND DECARBOXYLATION}

By E. A. Evans, Jr., University of Chicago . . . . . . . 197

Introduction: cycle reactions in biological systems, 197

Krebs' citric acid cycle, 198

Experimental basis of the citric acid cycle, 198

The "Krebs reaction," 200

The effect of malonate on tissue respiration, 201

Evaluation of the reactions of the citric acid cycle, 201

Criticisms of the theory, 202
Energy utilization mechanisms

By M. J. Johnson, 194

Evidence for acetylphosphate occurrence

By Fritz Lipmann, 195
The citric acid cycle in pigeon liver, 203

The synthesis of $\alpha$-ketoglutaric acid, 203

The assimilation of $\mathrm{CO}_{2}$ in the synthesis of $\alpha$-ketoglutaric acid by pigeon liver, 204

The mechanism of $\alpha$-ketoglutaric acid synthesis, 204

The nature of $\mathrm{CO}_{2}$ assimilation by pigeon liver, 205

\section{TRANSAMINATION}

By Philip P. Cohen, University of Wisconsin

Types of transamination, 210

Discovery of transamination reaction, 211

Substrates active in transamination, 211

Amino and keto acids, 211

Peptides, 212

"Primary" and "secondary" substrates, 213

"Catalytic" transamination, 213

Preparation and properties of transaminating enzymes, 214

Aminopherases, 214

Transaminase, 215

Substrate specificity, 215

Mechanism of transamination, 216

Transamination in different tissues: Animal tissues, 218
Malignant and embryonic tissues, 219

Plant tissues, 220

Yeast and bacteria, 221

Transamination in vivo, 221

Influence of various substances on transamination, 221

Inhibitors, 221

Hormones, 222

Carcinogens, 222

Vitamins, 222

Role of transamination in intermediary metabolism, 223

Protein and amino acid synthesis and degradation: animal tissues, plant tissues, transamination and glycolysis, transamination and hydrogen transport, 223 
Characteristics of Tumior ResPIRATION by K. A. C. Elliott, 229

Phosphorylation Theories and Tumor Metabolism by Van $R$. Potter, 233

On the Specificity of Glycolysis in Malignant Liver Tumors as Compared with Homologous
Adult or Growing Liver Tissues by Dean Burk, 235

The Effects of Certain Diamines on Enzyme Systems, Correlated WITI THE CARCINOGENICITY OF the Parent Azo Dyes by C. J. Kensler, 246

\section{DISCUSSION ON BACTERIAL RESPIRATION}

W. H. Peterson, chairman . . . . . . . . . . . 252

Criteria for Experiments with Isotopes discussed by H. G. Wood and R. H. Burris, 252

MechanisMS FOR THE COMPLETE Oxidation of Carbohydrates BY Aerobic Bacteria discussed by
C. H. Werkman, E. S. Guzman Barron, and P. W. Wilson, 258

Reactions in Cell-Free Enzyme Systems Compared with Those in the Intact Cell discussed by F. F. Nord and P. W. Wilson, 264

\section{DISCUSSION ON ANIMAL TISSUE RESPIRATION}

C. A. Elvehjem, chairman . . . . . . . . . . 268

Factors affecting the Preparation of Tissue for Metabolic Studies by Ephraim Shorr, 268

Comparison of Slices AND HomoGENized Suspensions of Brain Tissue by K. A. C. Elliott, 271

The Homogenized Tissue TechNique, the Dilution EfFect and Ion EFFects by Van R. Potter, 274 The Stimulatony Effect of CalCIUM UPON the Succinoxidase AC- tivity of Rat Tissues by A. E. Axelrod, 275

Tissue Metabolism in Vitro and IN Vivo by Frederick Bernheim, 276

Pathways of Carbohydrate MeTABOLISM by E. S. Guzman Barron, 278

The Citric Acid Cycle in Tissue Metabolism by Fredrick J. Stare, 280 


\title{
A SYMPOSIUM ON RESPIRATORY ENZYMES
}

\author{
Addresses given at an Institute \\ Held at the University of Wisconsin \\ September 11-17, 1941
}





\title{
Intermediate Carbohydrate Metabolism
}

\author{
OTTO MEYERHOF \\ University of Pennsylvania
}

7 HIS REVIEW OF THE intermediary carbohydrate metabolism must 1 necessarily be treated broadly and generally, for the subject has many different aspects, and the detailed questions of hydrogen transport, Pasteur effect, pyridine nucleotides, cocarboxylase, metabolic cycles, phosphorylations, indeed all the items which are intrinsic elements of the present picture of carbohydrate breakdown, will be dealt with by competent investigators of these subjects. Moreover, I had the opportunity to discuss the special question of oxidoreduction and dismutation in carbohydrate metabolism at the Chicago congress some months ago.

If we take this occasion to look back fifty years and to compare our present knowledge with that which existed at the end of the last century we have reason to be very proud, for at that time this whole field appeared nearly as tabula rasa. But two outstanding achievements had already been accomplished: first, Claude Bernard's work on the interconversion of glucose and glycogen in the liver and on the role of blood sugar under normal and diabetic conditions; second, the work of Pasteur on the different microbic fermentations as manifestations of the anaerobic metabolism of these organisms. Nothing was known about the oxidative breakdown of sugar. Although lactic acid formation in the blood and especially in the muscles had been observed by Claude Bernard and others, it was not known whether nor how this cleavage was connected with respiration.

Since then the interconversion of glycogen and blood sugar have continued to claim the attention of medical investigators, and recently, as you know, a highly interesting development was reported: Professor Cori's discovery of glucose-1-monophosphoric acid as intermediary. The old problem of diabetes was shifted by the isolation of insulin from the study of blood sugar regulation to the biochemical task of studying tissue metabolism under the influence of added hormones. The third old problem of the connection between 
fermentation and respiration remained for a long time a subject of speculation, and even now many a question is unanswered. However, I should like to follow this latter trend of ideas a little more in detail.

Pfeffer and Pflüger, following Pasteur, held a rather simple view of this relationship: the first step of respiration was assumed to be always anaerobic. If no oxygen is present, the products of anaerobic cleavage accumulate: alcohol in yeast and higher plants, lactic acid in the tissues of higher animals and in some bacteria. But if oxygen is present, these products are oxidized to carbon dioxide and water.

That this concept required modification became apparent twenty years ago from studies of metabolism of muscle. In 1907 Fletcher and Hopkins (1) showed that under anaerobic conditions frog muscles formed lactic acid steadily during both activity and rest, and that this lactic acid disappeared when oxygen was admitted. Parnas (2), working some years later in the same Cambridge laboratory, claimed to have found that this disappearance was a complete oxidation, thus apparently confirming the views of Pfeffer and Pflüger. In 1920, because of the controversial state of this question, I repeated the experiments of Parnas, avoiding especially all kinds of irritation or injury of the muscles which would lead to extra-consumption of oxygen (3). Under these conditions much more lactic acid disappeared in oxygen than could be accounted for by oxidation, and the lactic acid unaccounted for was reconverted into carbohydrate. This was true for the lactic acid formed during activity as well as for that formed during rest. Similarly, it was shown that in equal periods of rest much more lactic acid was formed anaerobically than could be burnt aerobically by the resting respiration. Indeed, the amount of oxygen which failed to be used in a period of anaerobiosis was about the same as the excess consumed after that period. This oxygen was sufficient only to oxidize from a quarter to a sixth of the lactic acid which disappeared.

These facts, which are independent of special interpretations, are sufficient to invalidate the original theory of Pfeffer and Pfluger in that they show that the oxidative removal of fermentation products is not necessarily identical with the oxidation of these products. But we can pose the more limited question whether the oxidation on the whole attacks the end products of anaerobic breakdown. With respect to lactic acid formed in a preceding anaerobic period, we must surely answer in the affirmative. We know that lactic acid is easily oxidized by way of pyruvic acid. For example, Barron et al. 
(4) showed that specially treated, washed bacteria may lose the power to oxidize sugar and other substrates, but retain the power to oxidize lactic to pyruvic acid. Experiments on muscle lead to the same conclusion. After a muscle is poisoned with iodoacetic acid the formation of lactic acid is blocked; at the same time the respiratory quotient drops to 0.7 , and is not changed by the addition of sugar, but is brought to 0.95 by the addition of lactic acid. Respiration is increased, and oxygen consumption is essentially equivalent to the disappearance of lactic acid (5). Similar results were obtained by Krebs with respiration of brain and testis after poisoning with iodoacetic acid (6). Since oxidation of sugar is completely checked, no interpretation is possible except that lactic acid is directly oxidized.

But this is not necessarily the pathway of sugar oxidation in the aerobic steady state. That independent ways of sugar oxidation exist may be gathered from many observations, such as the rapid oxidation of fructose in brain tissue, where, in contrast to glucose (7), it does not give rise to anaerobic lactic acid. Furthermore, Warburg and Christian showed that hexosemonophosphate can be oxidized by the triphosphopyridine nucleotide in yeast extract to phosphogluconic acid (8), and Lipmann demonstrated the complete oxidation to carbon dioxide in this manner (9).

On the other hand, the oxidation of sugar by way of pyruvic acid is also firmly established, and in this case the steps up to the formation of the acid are identical in respiration and in anaerobic glycolysis. As was discovered by Peters (10), pyruvic acid accumulates during oxidation of carbohydrate by cells and tissues in cases of vitamin $\mathrm{B}_{1}$ deficiency, which means that lack of cocarboxylase blocks the oxidative decarboxylation of pyruvic acid. Many other findings, such as the similarity of the oxidation of pyruvic acid to that of sugar in tissue pulps and extracts, point in the same direction, namely, that sugar is oxidized via pyruvic acid (11). Thus several pathways of sugar oxidation exist, the choice of which may depend upon the special set of enzymes in different tissues and also upon hormonal and other controlling influences.

All this probably has some bearing on the relationship already mentioned between oxidation and interference with the mechanism of fermentation. I have mentioned before the two possible cases of this relationship-the actual synthesis of split products to the initial substance and the non-formation of the split products during the stationary state of respiration. Without fearing to be accused of a 
biased judgment I dare say that both cases are characterized by the same numerical relationship-the oxidation quotient, which expresses the ratio of the aerobic disappearance of splitting metabolism in moles sugar to the oxidized sugar equivalents (12). Critics have objected that under extreme conditions this number may range from zero to infinite, but it is equally true, and more important, I think, that under physiological conditions living cells exhibit quotients between 3 and 6 -approaching 6 more and more as the conditions of temperature, oxygen pressure, nutritional state, and milieu become optimal for the cells in question. The same preference for the quotient of 6 was demonstrated by $O$. Warburg for different warm-blooded tissues where the anaerobic glycolysis is high enough to allow the calculation of the quotient (13).

The original concept of a metabolic carbohydrate cycle involved the assumption that in the stationary state the quotient results from a continuous overlapping of anaerobic glycolysis and of oxidative resynthesis of the cleavage products-the endothermic resynthesis made possible by coupling with oxidation. Today it seems possible to refine this scheme and to modify it somewhat without rejecting the main argument. Indeed, in the past fifteen years a tremendous amount of material has been collected to prove that the general concept of these cycles in carbohydrate breakdown holds good, that every oxidative step is coupled with an involuntary phosphorylation, and that the several intermediate stages of the anaerobic breakdown can be reversed by means of the "energy-rich phosphate bonds" (31) created in this way.

On the other hand, the original concept of a single complete cycle passing through the stage of lactic acid cannot be exactly true for a very simple reason, which has become clear since 1933; namely, that pyruvic acid is the necessary precursor of lactic acid in glycolysis and of alcohol in yeast fermentation (14). Under anaerobic conditions the reduction of pyruvic to lactic acid is compensated for by the oxidation of phosphoglyceraldehyde to phosphoglyceric acid. The latter, in turn, is decomposed via two intermediaries to pyruvic acid (15). The hydrogen transfer proceeds in both directions by the way of cozymase, the diphosphopyridine nucleotide of Warburg.

But if oxygen is present the dihydrocozymase can transfer its two hydrogen atoms to oxygen instead of to pyruvic acid by a long chain of oxidative catalysts: the pheohemin enzyme of Warburg, the three cytochromes, and the flavinproteins; consequently the pyruvic acid 
is not reduced. On the contrary, such an oxidation of dihydrocozymase shifts the equilibrium in the opposite direction, so that lactic acid, if present, would be oxidized by cozymase to pyruvic acid, whereas in the stationary state of sugar oxidation pyruvic acid would be continuously formed by way of phosphoglyceric acid, without a compensating reduction.

Therefore only pyruvic acid, and not lactic acid, is formed in the stationary state of oxidation. This interpretation at the same time gives a clue to the oxidation quotient, the numerical relationship between the oxygen consumed and the lactic acid that is prevented from being formed: if one atom of oxygen is required to oxidize the two hydrogen atoms of dihydrocozymase, then this atom prevents one molecule of pyruvic acid from being reduced to lactic acid or in yeast fermentation to alcohol. Therefore six atoms of oxygen (corresponding to the complete oxidation of one molecule of lactic acid) can prevent six molecules of lactic acid from being formed, and we obtain the normal oxidation quotient of 6 . Of course this refers only to the principle. The cozymase reoxidized by oxidative catalysts must dehydrogenate other intermediary stages besides triosephosphate, because every oxidative step in the breakdown of sugar acts in the same way, preventing the formation of one molecule of lactic acid per'one atom of oxygen taken up.

And this is only one side of the picture. If the breakdown of sugar in oxygen and in nitrogen proceeded with the same speed to the stage of pyruvic acid, and the only difference consisted in the fate of pyruvic acid to be reduced or further oxidized, then the oxidation would not prevent, as it actually does, by this so-called "Pasteur effect," the greater part of sugar from disappearing. But here the concept of metabolic cycles has its place. Actually every oxidative step is coupled with the phosphorylation of the adenylic system, and by this means a corresponding phase of anaerobic breakdown is reversed, so that for every oxygen atom consumed one three-carbon molecule can return to its initial stage as sugar or glycogen. This state of affairs is very neatly shown by the recent experiments of Cori, Kalckar, and co-workers (16) with dialyzed extracts of kidney and heart, and by experiments of Belitzer and Tzibakowa (17) with washed pigeon muscle. Cori and his group found that in the presence of the complete glycolytic coenzyme system the organ extracts oxidize glucose and phosphorylate an excess of it, so that for every hexose molecule burned to carbon dioxide, ten molecules of phosphate are taken up to form five molecules hexosediphosphate; and 
since the oxidized molecule also had to be phosphorylated, altogether twelve molecules of phosphates are taken up for one molecule glucose or twelve oxygen atoms consumed. Therefore every step of glucose oxidation consisting in an oxidoreduction between cozymase and an oxidizable intermediary is coupled with phosphorylation. Not only is this true for the two steps where it is already known, i.e., the oxidation of phosphoglyceraldehyde and that of pyruvic acid, in which Lipmann discovered acetylphosphate as the primary product of oxidation (18), but for every such step an energy-rich phosphate bond is created in adenosinetriphosphate, which enables a synthetic step to take place.

The experiments of Belitzer and Tzibakowa are a little different, because they added creatine to cut muscle and obtained under these conditions a synthesis of creatinephosphate when lactate, pyruvate, or the four-carbon acids of the Szent-György cycle were oxidized. At the most two molecules of creatinephosphate were formed for every oxygen atom taken up. Although the presence of creatine diverts the pathway of synthesis from carbohydrate, the experiments are important in that they demonstrate the uptake of two molecules of phosphate by way of adenosinetriphosphate for one atom of oxygen consumed; this relationship is comparable to the synthesis of creatinephosphate in muscle extract, where two steps of glycolysis are involved in the transfer of phosphate, namely, the oxidoreduction and the dephosphorylation of phosphopyruvic acid (19).

Moreover, the reaction studied by Belitzer is closely analogous to the recovery period of the living muscle, especially a muscle which is only slightly fatigued. Here, during oxidative recovery, the oxidation serves mostly for the resynthesis of creatinephosphate, and to a small extent for that of glycogen. If two molecules of creatinephosphate are synthesized for every atom of oxygen taken up, then about 40 per cent of the combustion heat of sugar or lactate is consumed for the endothermic synthesis, a result which comes very close to the efficiency of the oxidative recovery in the living muscle.

But to return from this digression to the significance, already mentioned, of the experiments for the theory of carbohydrate cycles. One objection may be raised against this interpretation of the Pasteur effect. Many cases are known where the respiration remains quantitatively the same, while the effect of the respiration on the

* Actually the same ratio of two molecules of creatinephosphate synthesized for one atom of oxygen taken up was found by O. Meyerhof and D. Nachmansohn (Biochem. Z., 222, 1, 1930) during recovery of a partially fatigued muscle. 
glycolysis is suppressed. In the picture outlined above the oxygen used would automatically eliminate an equivalent lactic acid formation, in so far as the oxygen serves to reoxidize dihydrocozymase. But we must have in mind that the oxygen intervenes only indirectly by way of the oxidizing catalysts. Here the so-called "Pasteur enzyme" assumed by Warburg (20) and demonstrated by Stern and Melnick (21) plays its role in steering the oxidation. All oxidation not going by the way of cozymase would be without "Pasteur effect"; it may be oxidation of non-carbohydrate, which replaces sugar oxidation, or it may be oxidation of sugar by way of triphosphopyridine nucleotide.

Now we come to the second half of the problem, the actual conversion of lactic acid to glycogen in the oxidative recovery of the

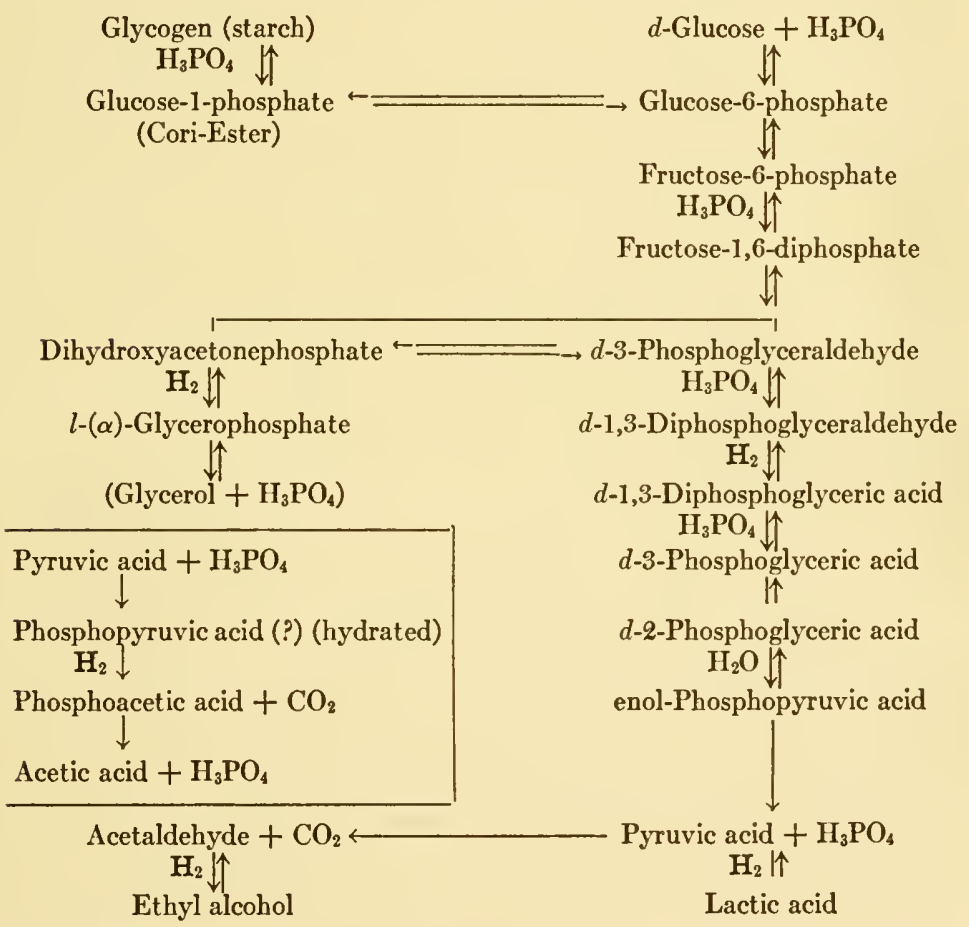

Figure 1.-Complete sequence of intermediaries in anaerobic breakdown of carbohydrate

Insertion on the left: oxidative decomposition in lactic acid bacteria, according to Lipmann (18). 
isolated frog muscle or in the mammalian liver. Without going into too much detail I will show you the table of consecutive intermediary steps, neglecting the coenzymes concerned. As you see from the double arrows in Figure 1, nearly all these reactions are reversible. The transformation of glucose-1-phosphate into glucose-6phosphate is also reversible, according to Cori's recent findings (22). This reversibility is especially conspicuous for the oxidative step,

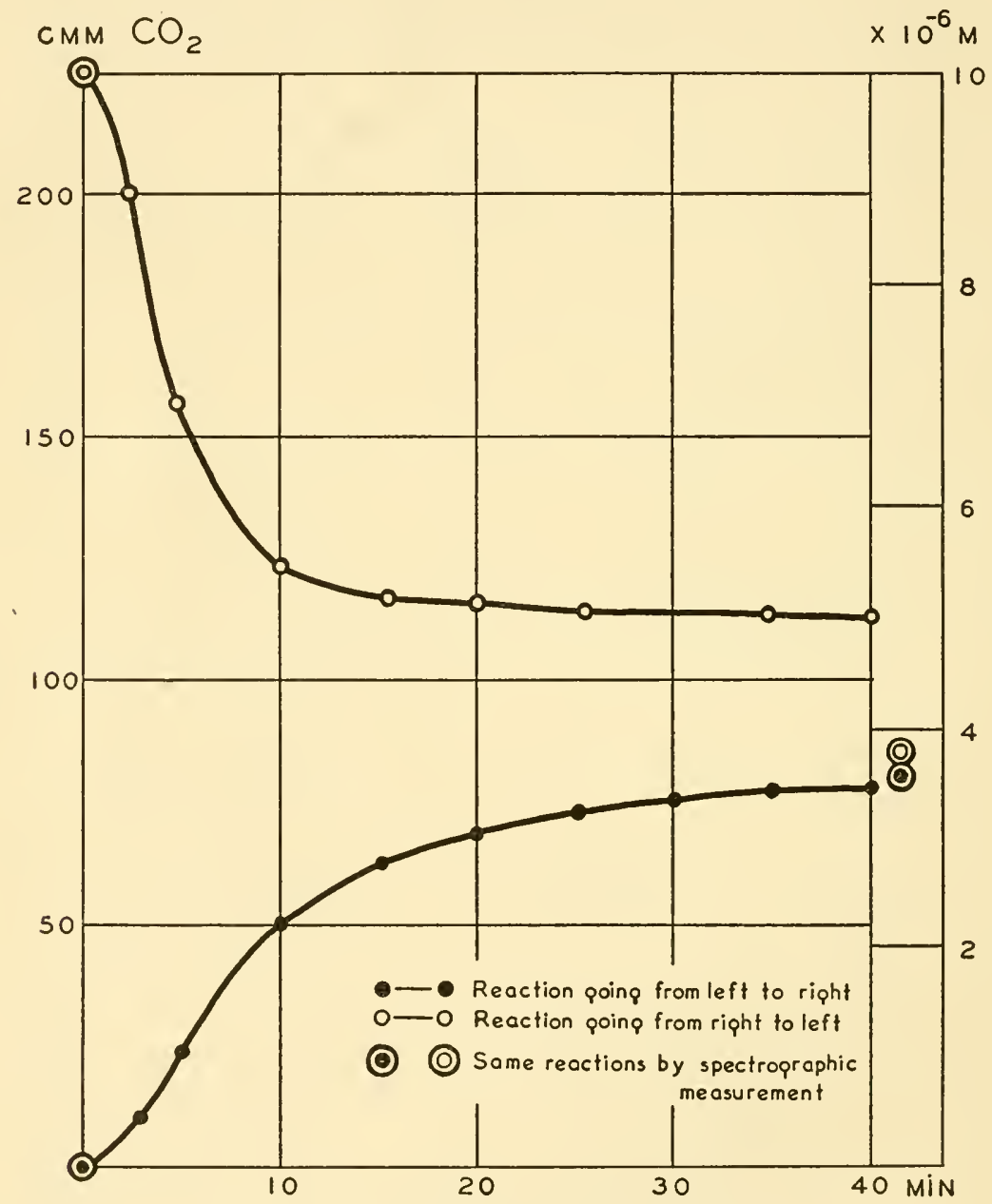

Figure 2.-Stoichiometric coupling reaction

Oxidation of phosphoglyceraldehyde is drawn upward. The points on the lines were obtained by manometric measurement. The spectrographic measurements were of dihydrocozymase (absorption maximum $340 \mathrm{m \mu}$ ). 
the oxidation of phosphoglyceraldehyde to phosphoglyceric acid. Even in the presence of a stoichiometric amount of cozymase, which is simultaneously reduced, the reaction would proceed completely in the direction of oxidation but for the coupling with phosphate uptake by the adenylic system. Thereby a measurable equilibrium is obtained, which was established in 1938 (23) in the Heidelberg Institute and which is shown in Figure 2. The kinetic nature of this equilibrium can be neatly demonstrated by the use of radioactive phosphorus. If radioactive inorganic phosphate is added to the enzymatic mixture after the equilibrium has been established, the adenosinetriphosphate in the solution rapidly takes up radioactive

$10^{-5} \mathrm{M}$

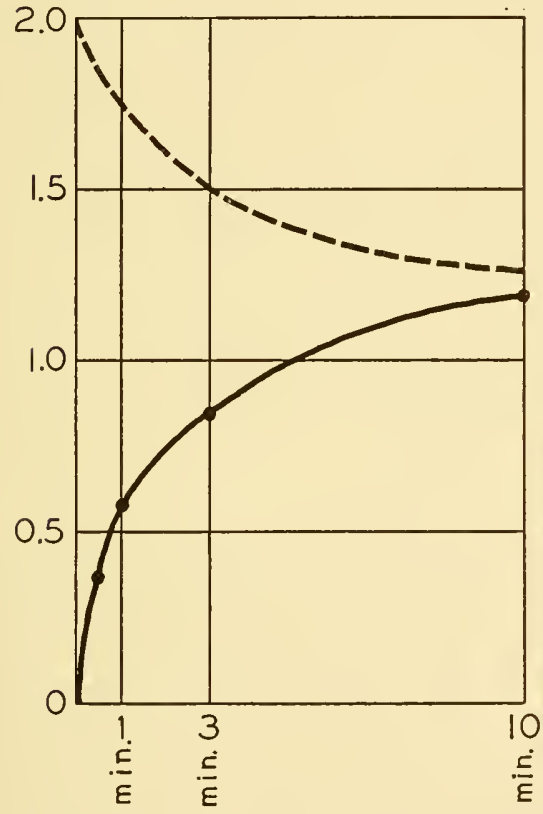

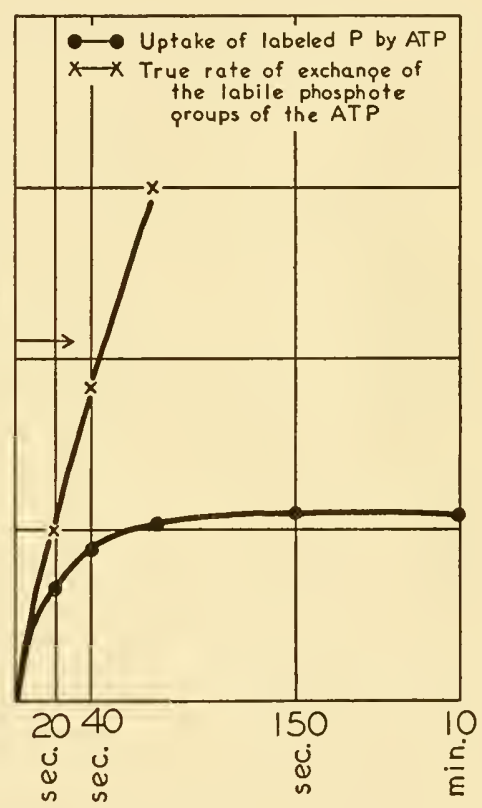

Figure 3.-Use of radioactive phosphate in the reversible coupling reaction Left: Adjustment of the equilibrium of the coupling reaction; Right: Adjustment of the equilibrium of the isotopes

phosphate, although no chemical change takes place (24), as shown in Figure 3. This reversible coupling reaction was confirmed and explained by Warburg, Christian, and Negelein in the way shown in Figure 4. They isolated the 1,3-diphosphoglyceric acid as inter-

* This substance is identical with the $d$-component of the "Fischer-Baer ester" (Chem. Ber. 65, 337, 1932). 
mediary, and consequently we have three consecutive steps (25). The overall reaction goes from left to right or in the opposite direction, depending upon the concentration of the reactants (Figure 4). If, for instance, adenosinetriphosphate is continuously resynthesized by an independent reaction, so that its concentration remains high and the concentration of the inorganic phosphate low, the reaction is pushed in the direction of the reduction of phosphoglyceric acid. This reaction is favored still further if the phosphoglyceraldehyde is removed by isomerisation to dihydroxyacetonephosphate and by condensation to hexosediphosphate and so on. In this way the synthesis can actually take place.

There remains one reaction which in the light of experiments with radioactive phosphorus, seems to be irreversible-the dephosphorylation of phosphopyruvic acid. Adenosinetriphosphate con-

I. Stoichiometric-coupled reaction (Meyerhof, Ohlmeyer, Kiessling, 1937-38) (Co=cozymase)

$d$-3-Phosphoglyceraldehyde $+\mathrm{Co}+$ Adenosinediphosphate $+\mathrm{H}_{3} \mathrm{PO}_{4} \rightleftarrows$ $d$-3-Phosphoglyceric acid $+\mathrm{CoH}_{2}+$ Adenosinetriphosphate

II. Warburg and Christian's explanation of the coupled reaction (1939)

A. d-3-Phosphoglyceraldehyde $+\mathrm{H}_{3} \mathrm{PO}_{t} \rightleftarrows 1,3$-Diphosphoglyceraldehyde (?)

B. 1,3-Diphosphoglyceraldehyde $+\mathrm{Co} \rightleftarrows 1,3-D i p h o s p h o g l y c e r i c$ acid $+\mathrm{CoH}_{2}$

C. 1,3-Diphosphoglyceric acid+Adenosinediphosphate $\rightleftarrows$

3-Phosphoglyceric acid+Adenosinetriphosphate

Figure 4--Coupling of phosphorylation and oxidoreduction

taining radioactive phosphorus in its labile groups did not exchange this radioactive phosphorus with phosphopyruvic acid. If the reaction between phosphopyruvic and adenylic acid were reversible, an exchange should have taken place. But we must concede that the experimental basis for this negative result is not too large and therefore accept it with some reservation until it is more firmly established. If for the moment we accept the result, it has important implications. There is indeed reason to believe that the oxidation of carbohydrate by way of the four-carbon acids intervenes to bring pyruvic acid back to phosphopyruvic. Some years ago Kalckar observed, during oxidation of fumarate by kidney extract, the formation of an acid which seemed phosphopyruvic (26). The more recent findings of several investigators $(27,28,29,30)$ on the assimilation of isotopic carbon dioxide, which appears in oxalacetic and ketoglutaric acid and in glycogen during synthesis from lactic acid, fit very well into such a scheme. Now that the condensation of carbon 
dioxide with pyruvic to oxalacetic acid has been proved by Evans and by Wood and Werkman, we have only to assume that the carbonyl group of the latter is phosphorylated, forming a phospho-enol oxalacetic acid; the phospho-enol group would then be equally distributed between the alpha and beta position and the compound again decarboxylated; so we obtain a phosphopyruvic acid, half of which contains labeled carbon in the carboxyl group. Such a reaction may well be coupled with oxidation of fumaric and malic acid to<smiles>CC(C)=O</smiles>

$\mathrm{COOH} \quad$ Oxalacetic Acid

Pyruvic Acid<smiles>CCCCCC[14CH3]</smiles>

Phosphoenoloxalacetic Acid

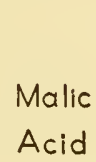

$$
\mathrm{COOH}
$$

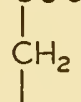

Acid<smiles>CC(O)C(=O)O</smiles>

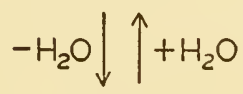<smiles>O=C(O)CCCCC(=O)O</smiles>

Fumaric

$\mathrm{CH}$

Acid<smiles>C=CC(=O)O</smiles>
pyruvic Acid

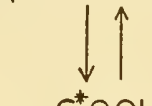<smiles>O=C(O)C(=O)C(=O)O</smiles>

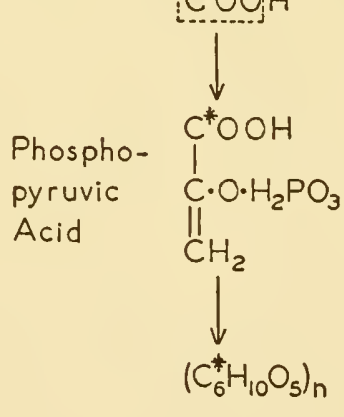<smiles>O=C(O)CCC(=O)O</smiles>

Figure 5.-Possible mode of uptake of carbon dioxide containing radioactive carbon $\left(\mathrm{C}^{*}\right)$ during synthesis of glycogen from lactic acid 
oxalacetic acid. These hypothetical reactions are shown in Figure 5. In this or in a similar manner, by the way of a phosphomalic acid, as Lipmann (31) and Hastings (28) recently suggested, the gap between pyruvic acid and enol-phosphopyruvic may be bridged with simultaneous oxidation of four-carbon acids. Indeed, with the closing of this gap the whole chain of reactions leading from lactic acid to glycogen will be completely understood. Furthermore, the real point of attack of insulin, which has so far eluded all investigators, may even be sought in the oxidative mechanism concerned with the four-carbon and five-carbon acids, lying on the pathway of oxidative sugar breakdown. Such an assumption, already proposed by Krebs (32), has not been conclusively proved by experiments.

Other questions concerning the intermediate metabolism of carbohydrate remain unsettled. One of these would seem to be relatively easy to attack with our present facilities. Fermentation is inhibited by not too high concentrations of cyanide, nitric oxide, hydrogen sulfide, and $o$-phenanthroline, substances known to form complexes with heavy metals. Such complex formation is responsible, as we know from the work of Warburg and many others, for the inhibition of respiration by these and similar substances. We cannot be sure at present whether this explanation holds good also for fermentation and glycolysis, since we do not know of any heavy metal indispensable to fermentation. Lohmann (33) of Heidelberg discovered that magnesium was essential for the phosphorylating enzyme system and for the carboxylase $(34,34 a)$, and magnesium can be replaced in many instances by still smaller concentrations of manganese (35). But it is doubtful whether the latter is the metal responsible for the inhibitions, and we do not know which of the many intermediary reactions these inhibitors attack. On the other hand, we know that fluoride attacks mainly the enolase, which dehydrates the 2-phosphoglyceric acid to enol-phosphopyruvic acid, while oxalate inhibits the dephosphorylation of the latter (36). But here too the mechanisms are unknown. Only in the case of iodoacetic acid, which affects the oxidoreduction steps in which cozymase takes part, does the mechanism of inhibition seem to be explained, namely, by the oxidation of the sulfhydryl groups of the dehydrogenase proteins (37).

Therefore our pride in the progress achieved in the last decades must be tempered by confession of ignorance regarding many crucial points. There are still many problems for this generation of research workers to solve. 


\section{REFERENCES}

1. Fletcher, W. M., and Hopkins, F. G., J. Physiol., 35, 247 (1907).

2. Pannas, J., Centralbl. f. Physiol, , 30, 1 (1915).

3. Meyerhof, O., Pflügers Archiv., 182, 232, 284; 185, 11 (1920).

4. Barron, Guzman E. S., and Miller, C. P., J. Biol. Chem., 97, 691 (1932).

5. Meyerhof, O., and Boyland, E., Biochem. Z., 237, 406 ( 1931 ).

6. Krebs, H. A., Biochem. Z., 234, 278 ( 1931 ).

7. Loebel, R. O., Biochem. Z., 161, 219 ( 1925).

8. Warburg, O., and Christian, W., Biochem. Z., 254, 438 (1932); 287, 440 (1936).

9. Lipmann, F., Nature, 138, 588 ( 1936).

10. Peters, R. A., Biochem. J., 31, 2240 ( 1937 ).

11. Colowick, S. P., Welch, M. S., and Cori, C. F., J. Biol. Chem., 133, 359, 641 (1940).

12. Meyerhof, O., Chemische Vorgänge im Muskel (Berlin, 1930).

13. Warburg, O., Posener, K., and Negelein, E., Biochem. Z., 152, 309 (1924).

14. Meyerhof, O., and Kiessling, W., Biochem. Z., 264, 40; 267, 313 (1933).

15. Meyerhof, O., Ergebnisse d. Physiol. (Asher-Spiro), 39, 10 ( 1937 ).

16. Colowick, S. P., Kalckar, H. M., and Cori, C. F., J. Biol. Chem., 137, $343(1940)$.

17. Belitzer, V. A., and Tziвakowa, E. T., Biokimia, 4, 516 (1939).

18. LipmanN, F., J. Biol. Chem., 134, 463 (1940).

19. Meyerhof, O., Schulz, W., and Schuster, P., Biochem. Z., 293, 309 (1937).

20. Warburg, O., Biochem. Z., 172, 432 ( 1926 ).

21. Stern, K. G., and Melnick, J. L., J. Biol. Chem., 139, 301 (1941).

22. Sutherland, E. W., Colowick, S. P., and Cori, C. F., J. Biol. Chem., 140, 309 ( 1941 ).

23. Meyerhof, O., Ohlmeyer, P., and Möhle, W., Biochem. Z., 297, 90, 113 (1938).

24. Meyerhof, O., Ohlmeyer, P., Gentner, W., and Meier-Leibnitz, H., Biochem. Z., 298, 396 (1938).

25. Warburg, O., and Christian, W., Biochem. Z., 303, 40 (1939).

Negelein, P., and Brömel, W., Biochem. Z., 303, 132 (1939).

26. Kalckar, H. M., Enzymologia, 2, 247 (1937); 5, 365 (1939); Biochem. J., 33, 631 (1939).

27. Ruben, S., and Kamen, M. D., Proc. Nat. Acad. Sci. (U. S.), 26, 418 (1940).

28. Solomon, A. K., Vennesland, B., Klemperer, F. W., Buchanan, J. M., and Hasting, A. B., J. Biol. Chem., 140, 171 ( 1941 ).

29. Evans, E. A., and Slotin, L., J. Biol. Chem., 136, 301 (1940).

30. Woon, H. G., Werkman, C. H., Hemingway, A., and Nier, A. O., J. Biol. Chem., 139, 365, 377, 483 (1941).

31. Lipmann, F., Advances in Enzymology, 1, 99 (New York, 1941).

32. Krebs, H. A., and Eggleston, L. V., Biochem. J., 32, 913 (1938).

33. Lohiman, K., Biochem. Z., 237, 445 (1931).

34. Lohman, K., and Schuster, P., Biochem. Z., 294, 188 ( 1937 ).

34a. Green, D. E., Herbert, D., and Subrahmanyan, V., J. Biol. Chem., 135, $795(1940) ; 138,327$ (1941).

35. Ohlmeyer, P., and Ochoa, S., Biochem. Z., 293, 338 ( 1937 ).

36. Lohman, K., and Meyerhof, O., Biochem. Z., 273, 60 ( 1934 ).

37. Rapkine, L., Biochem. J., 32, 1729 (1938). 


\title{
Oxidative Mechanisms in Animal Tissues
}

\author{
ERIC G. BALL \\ Harvard Medical School
}

IFE REQUIRES energy, and the study of life processes has resolved L itself largely into a study of various manifestations of the utilization of energy by the living organism. The source of this energy necessary for life was first indicated by the work of Lavoisier in 1770. Since then it has become increasingly recognized, as F. G. Hopkins has said, that "among the most fundamental of the dynamic chemical events related to life are the oxidations which yield energy to the cell."

Today we know a good deal about the oxidative processes taking place within the living cell, and we know a little about the amount of energy such processes may yield. We do not know, however, whether all the energy released by oxidative processes is utilized by the cell nor how it is utilized. Further knowledge concerning this aspect of the subject must perforce await fuller understanding of the mechanisms involved in the energy-yielding oxidative processes. That we are, however, upon the threshold of the solution is witnessed by the recent developments linking phosphorylation with oxidative processes in the living cell. It may well be that this symposium on respiratory enzymes and phosphorylation processes will mark a milestone in our advance. Let me, therefore, as my part in it, review briefly for you what we know today about the oxidative mechanisms in animal tissues and the energy they may yield.

Any consideration of the oxidative mechanisms in animal tissues has naturally centered about two points, oxygen and the organic substance undergoing oxidation. Outside the living cell oxygen does not react with the foodstuffs of the cell to any appreciable extent. Within the cell reaction occurs readily. This fundamental fact early suggested that within the cell either oxygen or the foodstuffs have become activated in some way that permits their interaction.

During the decade 1920-30 a controversy raged between two schools. One, championed by Warburg, claimed that oxygen activation was the all-essential mainspring. Once oxygen was activated, its direct attack upon the substrate was thought possible. The other 
school, headed by Wieland, claimed that activation of the substrate was most important. In particular, activation of the hydrogen of the substrate was stressed. Such activated hydrogen was believed capable of reacting with atmospheric oxygen to form water. Both schools seemed to agree that direct reaction between molecular oxygen and the substrate could occur. A release of energy in one tremendous burst was thus implied. As more data became available it became evident that both schools of thought were right and that biological oxidations took place only after both oxygen and the foodstuff to be burned were acted upon by intracellular enzymes. Within the last ten years we have also learned that it is doubtful whether any direct reaction occurs between oxygen and the substrate to be burned. Interposed between oxygen and the substrate are a series of so-called carriers through which electron exchange occurs, and energy is released in a series of successive steps.

Beginning with the oxygen end, let us examine the chain of events more closely. Evidence for the activation of oxygen has depended largely upon the use of so-called respiratory poisons. As a result of Warburg's earlier belief that iron in some form or other was the activator of oxygen, cyanide and carbon monoxide have become classical tools for the study of respiratory mechanisms. With the aid of these tools it has been proved, thanks to the laboratories of Warburg and Keilin, that at least four iron porphyrin compounds participate in biological oxidations. One of these, now commonly called cytochrome oxidase, is known largely as the result of its shadow-boxing with carbon monoxide. The other three, known

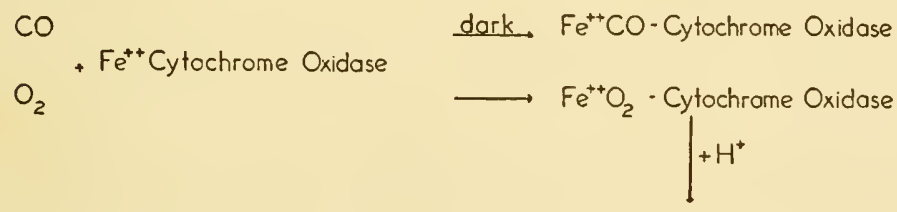

$\mathrm{Fe}^{+++} \mathrm{CN}$-Cytochrome Oxidase $\rightleftarrows \mathrm{CN}^{-}+\mathrm{Fe}^{+++}$Cytochrome Oxidase $+\mathrm{H}_{2} \mathrm{O}$

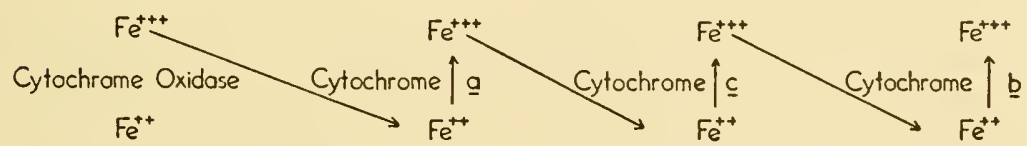

Figure 1.-The cytochrome system 
as cytochromes $a, b$, and $c$, are spectroscopically visible in their reduced forms. Their role in biological oxidations so far as we know it is shown in Figure 1. As depicted here, oxygen reacts with ferrous cytochrome oxidase, presumably to form an oxygenated compound similar to oxyhemoglobin, as evidenced by the competitive affinity shown by carbon monoxide. In the case of cytochrome oxidase, however, the oxygen is able to strike in and oxidize the ferrous iron to ferric. The role of oxygen in biological processes is now ended. Combining with hydrogen ions withdrawn from the acid-base continuum of the cell, it forms water. The ferric cytochrome oxidase thus formed can now bring about the oxidation of the cytochromes $a, b$, and $c$. In the presence of cyanide their oxidation is somehow prevented. Whether the cytochromes react as a chain or individually with ferric cytochrome oxidase we cannot say with certainty. If they react as a chain, we may align them as shown here in view of their relative oxidation-reduction potentials. The reaction involves the transfer of an electron from one iron compound to another without involving oxygen or hydrogen ions in the oxidation. Thus the oxidizing agent in the cell that we now have to deal with is ferric iron in organic combination. With what does it react? If we could answer that question, one of the largest gaps in our knowledge of the mechanisms of biological oxidations would be filled.

Since we can follow the pathway from the oxygen side no further, let us turn our attention to the substrate side, to the studies made upon its activation. The chief tools employed in these studies have been certain dyestuffs capable of undergoing reversible oxidation and reduction. Methylene blue in particular has been widely used; as we now know, its choice was a most fortunate one in view of the relative oxidation-reduction potentials of the systems concerned. By using methylene blue as the oxidizing agent in place of oxygen it was possible to show that the reducing action of various substrates can be elicited only when certain tissue constituents are also present. This was the most striking evidence that had been mustered for the view that substrate activation must take place in biological oxidations. In the hands of Thunberg and his co-workers this technique proved most useful in demonstrating the existence of a group of enzymes which were called dehydrogenases or dehydrases because their function appeared to be the activation of the hydrogen of the substrate in preparation for its removal to a suitable acceptor.

Each substrate or class of substrates, it was demonstrated, possesses its own specific dehydrogenase. Now since leuco-methylene 
blue is autoxidizable, it was possible in some cases to carry out the air oxidation of a substrate by the addition of its specific dehydrogenase and methylene blue. Such an oxidation was not, however, affected by cyanide or carbon monoxide and in this respect did not resemble the oxidation of the substrate by the living cell. As knowledge concerning the cytochrome system increased, it was soon realized that these iron porphyrin compounds played the role of methylene blue within the cells. Thus it was generally agreed about ten years ago that activation of the substrate was brought about by a specific dehydrogenase and that then the substrate reacted with oxygen through the cytochrome chain.

This, then, was the state of affairs in 1930 when Professor Warburg came to this country to deliver lectures on his work on what we now call cytochrome oxidase. Barron and Harrop (9) had shortly before published experiments showing that the addition of methylene blue to non-nucleated red blood cells brought about an oxygen consumption if glucose was present as a substrate. While Professor Warburg was at Johns Hopkins, Dr. Barron obligingly repeated his experiments at the request of his distinguished visitor, who watched the proceedings carefully. Upon his return to Germany, Warburg himself repeated the experiments and with his collaborators began the isolation of the red blood cell constituents responsible for this effect. Thus was begun a series of studies which brought forth some of the most noteworthy advances ever made in this field. As you know, these experiments led to the discovery of the vitamincontaining coenzymes essential to the functioning of most dehydrogenase systems. They showed that the activated hydrogen of the substrate did not react directly with the cytochrome system, but that at least two reversible oxidation-reduction systems were interposed.

So we have today the following general picture of the pathway of oxidations from the substrate side. In the presence of a specific protein and of a particular organic compound of low molecular weight, often called a coenzyme, the substrate loses two electrons and two hydrogen ions. In the majority of cases so far studied this coenzyme is one of the pyridine nucleotides. In these cases two electrons and one hydrogen ion are accepted by the pyridine nucleotide, the other hydrogen ion being released to the environment. We do not know whether both the pyridine nucleotide and the protein are concerned in the activation of the substrate molecule. If the protein alone is responsible for this activation, the pyridine nucleotide may 
be looked upon as a highly specific electron acceptor. Dixon and Zervas (11) favor this view and have presented evidence that several compounds can substitute for the pyridine nucleotide as electron acceptors. They have shown, for example, that alloxan can be reduced by malate or alcohol in the absence of the so-called pyridine nucleotide coenzyme, the specific protein alone being present. Whether alloxan plays such a role as an electron acceptor in living tissues is an open question. The observation of Jacobs (15) that the injection of alloxan into rabbits produced hypoglycemic convulsions is suggestive in view of the role of the pyridine nucleotides in sugar metabolism. Further evidence that the pyridine nucleotides are highly specialized electron acceptors is the fact that they participate in reactions in which the substrates possess markedly different chemical properties. Moreover, they show no great affinity for the specific protein also concerned in the reaction. Whatever their role, we know that the result of the reaction is the reduction of the pyridine nucleotide and the formation of the oxidized product of the substrate. The latter may in turn act as the substrate in another oxidation in which the same pyridine nucleotide is involved but with another specific protein.

Now in order that the pyridine nucleotide may act as a catalyst the reduced form must be reoxidized. The reduced pyridine nucleotides are, however, not unlike the substrates themselves in that they react sluggishly with most oxidizing agents. In the living cell they appear to be oxidized readily by only one specific class of substances, the flavoproteins. In this reaction two electrons and one hydrogen ion from the reduced pyridine nucleotide are transferred, along with a hydrogen ion from the environment, to the flavin portion of the flavoprotein. Whether the protein part of the flavoprotein functions by activating the sluggish reduced pyridine nucleotide is not known, though these flavoproteins might well be classified as reduced pyridine nucleotide dehydrogenases. It is worth noting that the electron acceptor is now firmly attached to a protein molecule as in the case of the cytochromes. The pyridine nucleotides thus occupy a unique position as electron acceptors in that they exist largely in the free state. This fact undoubtedly enables them to play their important role in anaerobic oxidation-reduction reactions.

The flavoproteins reduced by the pyridine nucleotide must now in turn be oxidized. This can be accomplished by methylene blue in the case of the isolated systems. In the intact cell, however, this cannot be the pathway. The direct oxidation of flavoproteins by 
molecular oxygen is too slow to be of physiological significance; moreover, none of them except one recently isolated from yeast by Haas, Horecker, and Hogness (14) reacts rapidly with cytochrome $c$. How then are they linked to oxygen in the living cell? This question we cannot at present answer. We are thus left with a gap between the flavoproteins on the one hand and the cytochromes on the other.

How large is this gap? Let us attempt to answer this question by considering the various known systems in our chain in relation to

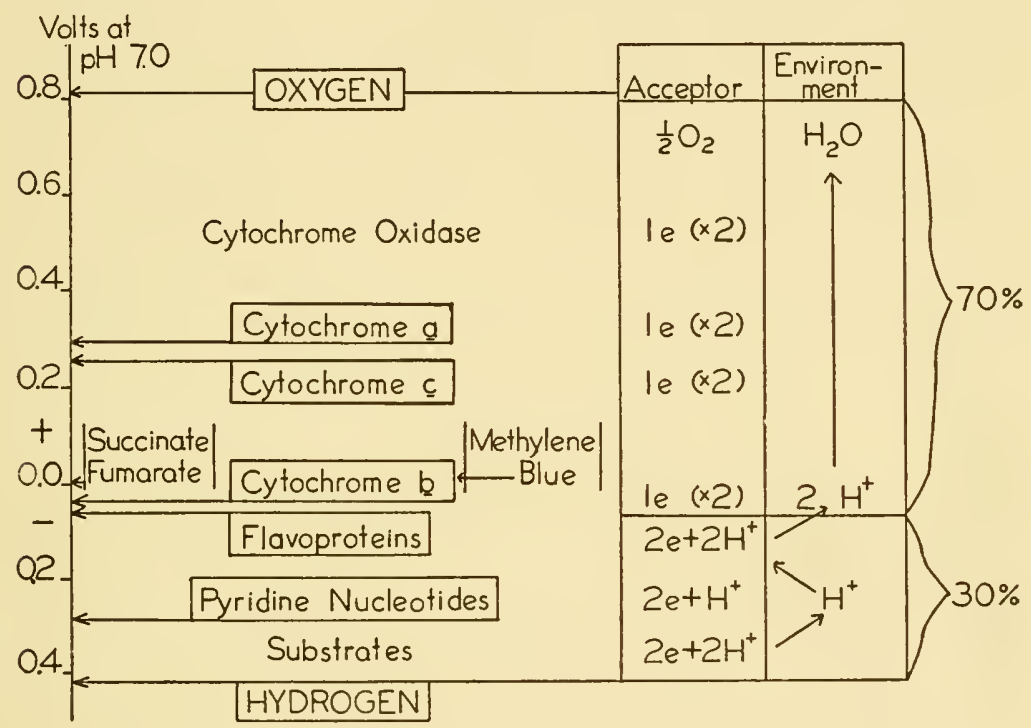

Figure 2.-Oxidation-reduction systems concerned in biological oxidations The source of the potential values used is given in reference 2 except for the diphosphopyridine nucleotide system, which is taken from reference 5 .

their oxidation-reduction potentials. As shown in Figure 2, we are able to plot fairly accurately according to their potentials all the systems discussed above. Cytochrome oxidase is the chief exception, but presumably we may place it between cytochrome $a$ and oxygen. We thus have interposed between the substrate and oxygen, reading in the order of the potential of their systems, pyridine nucleotides, flavoproteins, cytochrome $b$, cytochrome $c$, cytochrome $a$, and finally cytochrome oxidase. Now if cytochrome $b$ functions in this chain, and it must be remembered that we are not certain that it does, the possibility that another system lies between it and the flavo- 
proteins seems rather small. At any rate, the gap between these two systems in terms of the energy that would be released upon their interaction is only a small fraction of the overall. There seems little doubt, however, that cytochrome $c$ and the flavoprotein systems are necessary links in the chain. No flavoprotein has yet been obtained from animal tissues that will react directly with cytochrome $c$. This implies that there is some link in the chain between these two constituents. Until cytochrome $b$ can be isolated its claim to this position must remain in dispute. Another system that we have not yet mentioned must also be considered in this connection. SzentGyörgyi has suggested that the succinate-fumarate system links the flavoprotein systems to the cytochromes. It will be seen that the potential of the succinate-fumarate system is such that it could play this role. Here again, however, clean-cut proof is lacking, for succinic dehydrogenase and the cytochrome system appear to be intimately tied together and have so far defied separation. Permit me in passing to call your attention to the position of the methylene blue system. Situated as it is at this crossroad, it is well adapted to react with the flavoproteins on the one hand and on the other to bypass the cytochrome system in reacting with oxygen.

The chief pathway, then, by which energy is released in the living cell, so far as we can tell today, appears to be that shown here. The energy liberated when substrates undergo air oxidation is not liberated in one large burst, as was once thought, but is released in stepwise fashion. At least six separate steps seem to be involved. The process is not unlike that of locks in a canal. As each lock is passed in the ascent from a lower to a higher level a certain amount of energy is expended. Similarly, the total energy resulting from the oxidation of foodstuffs is released in small units or parcels, step by step. The amount of free energy released at each step is proportional to the difference in potential of the systems comprising the several steps. As indicated in this diagram, the steps involving the cytochromes account for more than two-thirds of the total energy released by this chain.

Now also, just as each lock in a canal must be passed in sequence, so here each link in the chain appears to be indispensable. Each component of the chain seems to react readily only with that component lying immediately above or below it. This marked specificity of interaction is most extraordinary in view of the fact that these substances may react with oxidizing and reducing agents foreign to the living cell. Methylene blue has already been given as an example 
of such a reaction occurring with the flavoproteins. Another example is the catalysis by the cytochrome system of the oxidation of certain organic substances, such as $p$-phenylenediamine. It should be noted, however, that such extraneous substances or their oxidized products may by their lack of specificity react in a way that is harmful to the cell mechanism. Methylene blue, for example, though not reduced by the $d$-amino acid oxidase system, gradually inactivates it in the presence of light (6). Mr. Kerr, working in my laboratory, has recently been investigating the mode of action by which butter yellow produces liver tumors. He has found that the oxidation catalyzed by a heart muscle preparation of extremely small quantities of $p$-phenylenediamine (an apparent breakdown product of butter yellow) completely inactivates the succinic oxidase activity of such a preparation. Thus the marked specificity of interaction of these compounds may also serve to prevent unwanted and harmful reactions from occurring within the cell.

Next arises the question whether the energy released at each step in this chain is utilized by the living organism and if so, how. At present direct evidence for the utilization of energy furnished by individual oxidative processes such as these is limited to the demonstration of coupled phosphorylation reactions. Such demonstrations have thus far been largely confined to that portion of the chain involving the pyridine nucleotides. Whether the large bulk of energy release that occurs through the cytochromes is useful for phosphorylations or for energy-utilizing mechanisms other than phosphorylation has not yet been definitely ascertained. It should be noted, however, that Korr (16) has pointed out that in the fertilized arbacia egg respiratory and functional activity are both inhibited by cyanide. Restoration of the respiratory rate by the addition of a substance such as methylene blue to replace the inactivated cytochrome system does not, however, restore functional activity.

A point that should perhaps be mentioned in connection with this pathway is the use of the term "hydrogen transport" to describe biological oxidations. $\mathrm{E}_{\mathrm{o}}^{\prime}$ values of the cytochrome $c$ system exhibit a zero slope in the neutral $\mathrm{pH}$ region. Preliminary experiments of the author indicate the same to be true for the cytochrome $a$ and $b$ systems. This indicates that only electron transfer occurs with these systems and that hydrogen is not concerned in the reaction. If the cytochrome oxidase system behaves similarly, only a fraction of the total energy released in biological oxidations involves hydrogen transport. Thus only the electrons of the substrate can be con- 


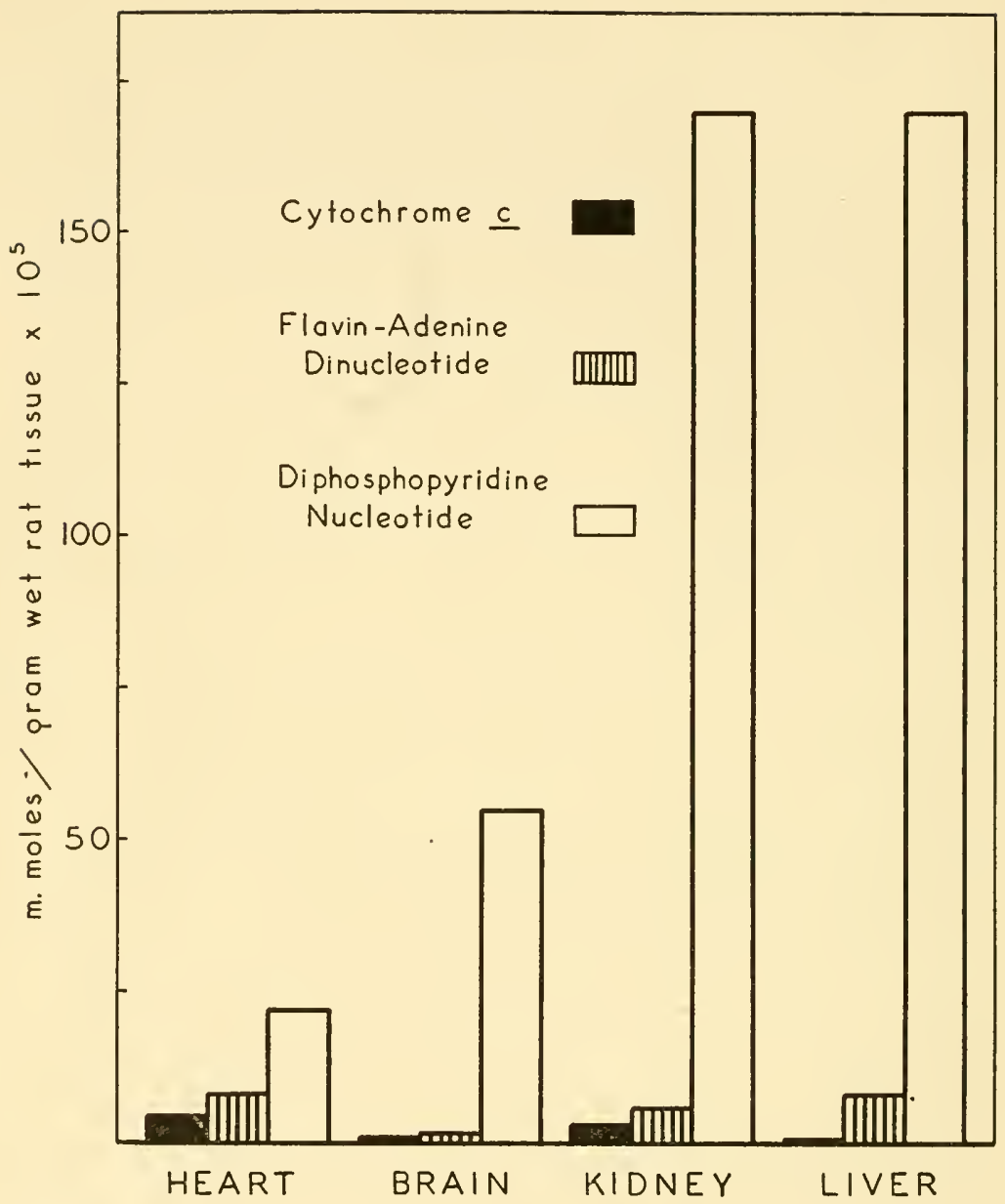

Figure 3.-The cytochrome $c$, flavin-adenine dinucleotide, and diphosphopyridine nucleotide content of four rat tissues

Values for the cytochrome $c$ content are recalculated from those given by Stotz (24) by assuming a molecular weight of 13,000. Flavin-adenine dinucleotide values are calculated from the data of Ochoa and Rossiter (22) and Warburg and Christian (25). Diphosphopyridine nucleotide values are calculated from the data of Axelrod and Elvehjem (1) with the exception of that for heart muscle, which is from a value given by von Euler et al. (12). The values given by von Euler $e t a l$. are much lower than those reported by other workers. 
sidered to be transferred to oxygen in an unbroken chain by the various acceptors. Hydrogen as hydrogen ion may enter or be withdrawn from the acid-base continuum at several places in the chain. Also, the various components of the chain may be classified in two groups according to their ability to transport electrons. The cytochromes can transport only one electron for each cycle of oxidation and reduction of their prosthetic groups. The functional groups of the flavoproteins and the pyridine nucleotides are capable, however, of transporting two electrons for each cycle. Thus the possible interaction of the cytochrome system with a flavoprotein would be one where a two-step, one-electron transfer, with the formation of a semiquinone flavoprotein intermediate, might play an important biological role. The ability of the free flavins to undergo such a stepwise oxidation has been amply demonstrated by the work of Michaelis and Schwarzenbach (21).

Now a consideration of the pathway just outlined might suggest that tissues contain each of these constituents in somewhat similar amounts. That such is not the case can be seen from Figure 3.

The cytochrome $c$, flavin-adenine dinucleotide, and diphosphopyridine nucleotide content of four tissues from the rat are here plotted in terms of millimoles per gram of wet tissue. The concentration of diphosphopyridine nucleotide in all four tissues is far greater than that of the other two constituents. Cytochrome $c$ is present in lowest concentration in all these tissues. In liver, for example, the concentration of the pyridine compound, expressed on a millimolar basis, is 340 times that of cytochrome $c$. Since cytochrome $c$ transports only one electron per mole, this ratio becomes $680: 1$ when expressed in terms of equivalents. In the other tissues the ratio is lower. From such relationships one might conclude that the cytochrome system is far more efficient in the transport of electrons than the other systems. Such indeed may be the case. A different explanation, however, is supported by more experimental proof, namely, that the pyridine nucleotides and the flavoprotein systems are involved in reactions other than those concerned in the main oxidative pathway. The known role of the pyridine nucleotides in certain anaerobic cycles is discussed elsewhere. With respect to the flavoproteins, recent studies have indicated that some of them are concerned in oxidative reactions which do not require the cytochrome system. Substrates such as the $d$-amino acids, hypoxanthine, xanthine, and certain aldehydes are so oxidized. The flavoproteins concerned in these reactions are unusual in that their 
reduced forms react directly with oxygen at a rapid rate. Thus they differ in this respect from the flavoproteins responsible for the oxidation of the reduced pyridine nucleotides. The reduced form of the $d$-amino acid oxidase flavoprotein also differs from all other flavoproteins in that it does not react with methylene blue or with dyestuffs of even higher oxidation-reduction potential (6). This variation in the behavior of different flavoproteins containing the same prosthetic group resembles the variation in the behavior of the various iron porphyrin protein compounds. Obviously the protein partner exerts a marked influence on the behavior of the prosthetic group.

Now such flavin systems as those just mentioned are probably of minor importance in furnishing the energy required by the cell; it can be shown that they are not affected by cyanide, which blocks the bulk of the oxygen consumption of the cell. In fact, these systems might be looked upon as incinerators for disposing quickly of unwanted products. Franke and Hasse (13) have termed them "rudimentary." It may be that they represent the earliest types of mechanism to emerge for the furtherance of biological oxidations and thus might be classed as primitive. They differ from cytochrome-linked systems in that hydrogen peroxide appears as a by-product of their reaction with oxygen. Thus there arises the question of the relationship of catalase and peroxidase to such systems. It is interesting to note that, of the tissues examined, the liver, among the richest in catalase, has the highest flavin and the lowest cytochrome $c$ content.

Finally, one other interpretation of the relatively low cytochrome $c$ content of tissues must be considered. This is the possible existence of pathways as yet unidentified which parallel the cytochrome system or supplement it. The existence of such unknown pathways has already been postulated as a result of certain experimental data obtained from a study of the action of inhibitors of respiration that are believed to poison cytochrome oxidase. Two of the most commonly employed inhibitors of this type are cyanide and azide. Let us consider, therefore, two examples of experiments involving the use of these respiratory poisons and examine the validity of the conclusions that may be drawn from such experiments.

The respiration of the unfertilized arbacia egg is insensitive to cyanide. Upon fertilization the egg consumes oxygen at a markedly increased rate, and the additional oxygen consumed is found to be cyanide-sensitive. These facts have been interpreted to indicate 
that in fertilized and unfertilized eggs the pathways for the oxidation of substrates are different. It should be noted, however, that at best the evidence merely indicates the possible existence in unfertilized eggs of a system alternate to the cytochrome system. Cyanide and azide do not effect the reduction of methylene blue by substrates acting through the pyridine nucleotide and flavoprotein systems. Hence these systems may be functioning in the unfertilized egg. Supporting evidence is furnished by the findings of Krahl et al. (18) that the unfertilized egg contains flavin-adenine dinucleotide and is also rich in diphosphopyridine nucleotide (17). Now does the cyanide insensitivity of the unfertilized egg indicate that an iron porphyrin system is not functioning in its respiratory mechanisms? Such an interpretation is indeed possible. One may, for example, postulate that the respiration is of a primitive type and passes directly through a flavoprotein to oxygen. Such a contention is supported by the fact that the presence of cytochrome $a, b$, or $c$ cannot be demonstrated in the unfertilized arbacia egg. It is possible, however, to demonstrate in the egg the presence of hemin substances (4). Moreover, Krahl and his co-workers (19) have recently shown that the eggs contain a substance resembling cytochrome oxidase, in amounts equal in activity to the cytochrome oxidase of mammalian tissues. This substance was found to be cyanide-sensitive if it was functioning in an oxidation requiring the addition of cytochrome $c$. Are we to conclude, then, that this egg "cytochrome oxidase" plays no role in the respiration of the unfertilized egg because such respiration is cyanide-insensitive? Is it not possible that a reaction can occur directly between "cytochrome oxidase" and flavoprotein in the arbacia egg? In view of the wide variety of properties exhibited by flavoproteins such a reaction might well occur. But it appears that such a postulate is contradicted by the evidence cited above that this egg "cytochrome oxidase" can be inhibited by cyanide.

Before we decide what is the correct interpretation of these data, let us review our knowledge about the mechanism of cyanide poisoning. Cyanide apparently inhibits respiration by reacting with cytochrome oxidase, since it prevents the air oxidation of the three reduced cytochromes. At low concentrations it apparently does not combine with the cytochromes. Presumably it combines with the ferric form of cytochrome oxidase, since it is methemoglobin and not hemoglobin that reacts with cyanide. We may conclude, then, that somehow cyanide prevents the reduction of ferric cytochrome oxi- 


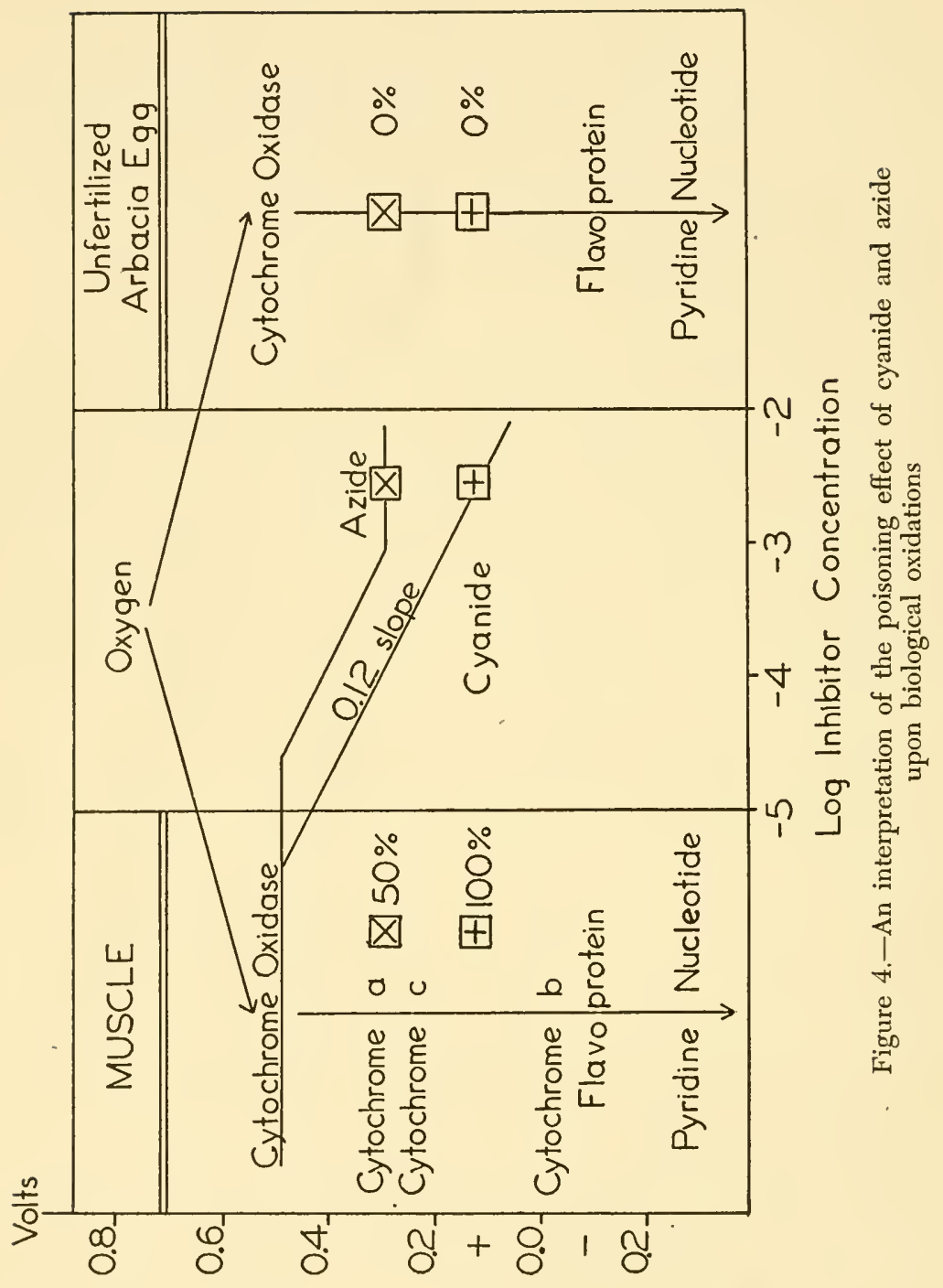


dase by the cytochromes. Now Barron (8) has presented ample evidence that cyanide hemochromogens are able to act as reversible oxidation-reduction catalysts. If the iron in such cyanide complexes can be reduced, why is the ferric iron of the cyanide complex of cytochrome oxidase not reduced?

As one answer to this question I suggested several years ago (3) that the oxidation-reduction potential of the cytochrome oxidase system is lowered in the presence of cyanide below that of cytochrome $a$ or $c$. The reduction of cytochrome oxidase by these compounds could then not occur and the respiratory chain as a whole might thus be blocked. The experiments of Barron (8), which showed that the cyanide hemochromogen systems were the most negative of the hemochromogen systems investigated, were cited in support of this idea. Since then Clark and his associates (10) have presented their thorough analysis of such hemochromogen systems. As a result of this study it may be said that the potentials of iron porphyrin systems vary according to the type of nitrogen compound associated with them. Also, if the affinity of the nitrogenous compound is greatest for the ferric form, the potential of the system will decrease progressively as the concentration of the coordinating compound is increased. The reverse holds true if the ferrous form displays the greatest affinity. Now assuming that the cytochrome oxidase system behaves in a similar manner, we may picture the effect of cyanide on the potential of the cytochrome oxidase system as shown in Figure 4. Here the cytochrome oxidase system is arbitrarily assigned a potential of 0.5 volts at $\mathrm{pH}$ 7.0. The potential of this system is plotted against the log of the concentration of the coordinating compound, as is done by Clark et al. (10) for the hemochromogen systems. It is assumed that only the ferric form of cytochrome oxidase reacts with cyanide and that since the first noticeable effects of cyanide poisoning result at concentrations of $10^{-5}$ to $10^{-6}$ molar, it is within this range that a potential shift will first occur." The potential is assumed to change according to a 0.12 slope, since this value has been found to hold for certain hemochromogen systems (10). It will be seen that, according to such a scheme, when the concentration of cyanide reaches $10^{-3}$ molar, the potential of the

* As shown by Clark et al. (10) for hemochromogen systems, this point depends also upon the concentration of the hemochromogen system. The concentration of the cytochrome oxidase system is of course not known. It is probably of the order of magnitude of the systems studied by Clark et al. (10) if the concentration of cytochrome $c$ in the tissues can be used as an index. 
cytochrome oxidase will be depressed below that of the cytochrome $a$ and $c$ systems. In such a tissue as muscle, then, we might expect 100 per cent inhibition of the cytochrome-mediated respiration. It is at such a concentration that cyanide exerts its maximum effect upon respiration.

Let us now return to the case of the unfertilized arbacia egg, where the cytochromes appear to be absent. Here cytochrome oxidase may be considered to react directly with a flavoprotein. The addition of cyanide may be pictured as depressing the potential of the cytochrome oxidase in the same manner. In this case, however, the potential of the cytochrome oxidase still remains above that of the flavoprotein with which it normally reacts. Oxidations can therefore still proceed, and no cyanide inhibition is observed. In fact, a stimulation of respiration by cyanide such as was observed by Marsh and Goddard (20) in the fully mature leaf might be encountered if such a set of conditions exists. Barron (7) has shown that the rate of reaction of oxygen with reduced dyestuffs increases as the oxidation-reduction potential of the system decreases. A similar change may occur in the case of the cytochrome oxidase system as its potential is lowered by cyanide.

Fertilization of the arbacia egg may then be looked upon as the gearing of the cytochrome oxidase system to another system of higher potential than the flavoprotein, which, though serving to increase the respiratory rate, also causes the system to become cyanide-sensitive. The fact that arbacia sperms are rich in cytochromes $a, b$, and $c$ is of interest in this connection. For a further discussion of this aspect see Krahl et al. (19).

One other example of an inhibitor study which may be employed is furnished by the work of Stannard (23). This investigator has recently presented data to show that resting and stimulated frog muscle respond differently to cyanide and azide. Whereas cyanide inhibits the oxygen consumption of both resting and stimulated muscle, azide inhibits only the oxygen consumption of stimulated muscle. The results have been interpreted to indicate that the pathways of oxidation are different in resting and in stimulated muscle. As Stannard points out, the cyanide sensitivity of the resting respiration seems to preclude the possibility that it represents an independent functioning of a flavoprotein system.

Now azide is believed to inhibit respiration also by reacting with cytochrome oxidase. Quantitatively, however, its action is different from that of cyanide. Inhibition of respiration by azide appears to 
begin at concentrations higher than those required for cyanide poisoning. As the azide concentration is increased above this initial value, inhibition progresses as rapidly as with cyanide until a concentration of about $10^{-3}$ molar is reached. Further increase in azide concentration produces no further effect, and inhibition of respiration remains incomplete, never reaching the maximum value obtained with cyanide. How then can this difference in the behavior of cyanide and azide be explained if they both act on cytochrome oxidase? Is it necessary to postulate that azide and cyanide inactivate separate systems?

An explanation of the difference in the behavior of cyanide and azide may be given in terms of their different effects upon the potential of the cytochrome oxidase system. Azide, like cyanide, is assumed to combine with the ferric form of cytochrome oxidase. Unlike cyanide, it is also assumed to combine with the ferrous form. In Figure 4 the effect of azide upon the potential of the cytochrome oxidase system is plotted on the basis of these assumptions. Since azide inhibition first manifests itself at concentrations higher than those for cyanide, its affinity for the ferric form of the oxidase is assumed to be less than that of cyanide. Depression of the potential of the oxidase system is therefore portrayed as starting when the concentration of azide reaches a value between $10^{-4}$ and $10^{-5}$ molar. As the concentration of azide is increased above this value, the potential is assumed to be lowered along the same slope as for the cyanide system. Now since inhibition with azide reaches a maximum at a concentration of $10^{-3}$ molar, it is assumed that at this concentration the azide begins to combine with the ferrous form of cytochrome oxidase. According to Clark et al. (10), the effect of such a combination on the potential of the system will be to alter the slope to a 0.0 value, and thus no further change in potential occurs as more azide is added. On the basis of this assumption it can be seen that, as depicted in Figure 4, the potential of the cytochrome oxidase system in the presence of azide can be depressed only to a level corresponding to that for the cytochrome $a$ system. At this level the cytochrome oxidase may still function to oxidize the cytochromes, but its efficiency will be at least 50 per cent impaired. It is thus possible to conceive of the respiration of resting muscle as being unimpaired by azide, since even at a lower level of efficiency the cytochrome oxidase may still be capable of supplying the oxygen needs of the resting muscle. If, however, increased demands for oxygen are made upon the muscle by stimulation, the impaired 
efficiency of the cytochrome oxidase system becomes a limiting factor.

By this time I can hear you muttering, "Sheer speculation," and I agree with you. I have, however, labored my point concerning the action of such inhibitors because I believe that until we know more about their mode of action any hypotheses of the existence of other oxidative pathways must also be labeled speculation. I do not wish to imply that other unknown pathways do not exist. I heartily agree that they may. I only beg that, in these days of ever-widening use of such inhibitors, more fundamental investigations be made into their mode of action before we becloud the issue with false interpretations.

\section{REFERENCES}

1. Axelrod, A. E., and Elvehjem, C. A., J. Biol. Chem., 131, 77 (1939).

2. Ball, E. G., Symposia on Quantitative Biology, Cold Spring Harbor, 7, 100 (1939).

3. BALI, E. G., Discussion published as part of reference 8 .

4. Ball, E. G., and Meyerhof, B., J. Biol. Chem., 134, 483 (1940).

5. Ball, E. G., and Ramsdell, P. A., J. Biol. Chem., 131, 767 (1939).

6. Ball, E. G., and Ramsdell, P. A., unpublished experiments.

7. Barron, E. S. G., J. Biol. Chem., 97, 287 (1932).

8. Barron, E. S. G., Symposia on Quantitative Biology, Cold Spring Harbor, 7, 154 (1939).

9. Barron, E. S. G., and Harror, G. A., Jr., J. Biol. Chem., 79, 65 (1928).

10. Clark, W. M., Taylor, J. F., Davies, T. H., and Vestling, C. S., J. Biol. Chem., 135, 543 (1940).

11. Dixon, M., and Zervas, L. G., Biochem. J., 34, 371 (1940).

12. von Euler, H., Schlenk, F., Heiwinkel, H., and Högberg, B., Z. physiol. Chem., 256, 208 (1938).

13. Franke, W., and IHasse, K., Z. physiol. Chem., 249, 231 (1937).

14. HaAs, E., Horecker, B. L., and Hogness, T. R., J. Biol. Chem., 136, 747 (1940).

15. Jacobs, H. R., Proc. Soc. Exp. Biol. Med., 37, 407 (1937).

16. Korr, I. M., Symposia on Quantitative Biology, Cold Spring Harbor, 7, 120 (1939).

17. Krahl, M. E., Verbal communication at a seminar on August 26, 1941, at Marine Biological Laboratory, Woods Hole, Mass.

18. Krahl, M. E., Keltch, A. K., and Clowes, G. H. A., Proc. Soc. Exp. Biol. Med., 45, 719 (1940).

19. Krahl, M. E., Keltch, A. K., Neubeck, C. E., and Clowes, G. H. A., J. Gen. Physiol., 24, 597 (1941).

20. Marsh, P. B., and Goddard, D. R., Am. J. Botany, 26, 724 (1939).

21. Michaelis, L., and Schwarzenbach, G. J., J. Biol. Chem., 123, 527 (1938).

22. Оснол, S., and Rossiter, R. J., Biochem. J., 33, 2008 (1939).

23. Stannard, J. N., Symposia on Quantitative Biology, Cold Spring Harbor, 7, 394 (1939).

24. Stotz, E., J. Biol. Chem., 131, 555 (1939).

25. Warburg, O., and Christinn, W., Biochem. Z., 298, 150 (1938). 



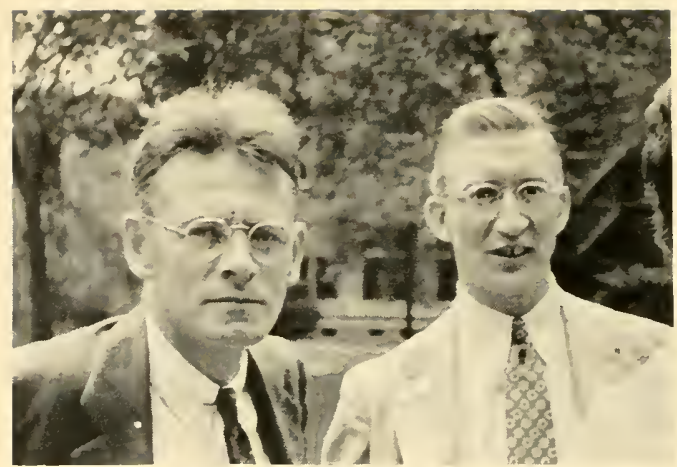

BALL AND STOTZ

From flavoprotein to cytochrome

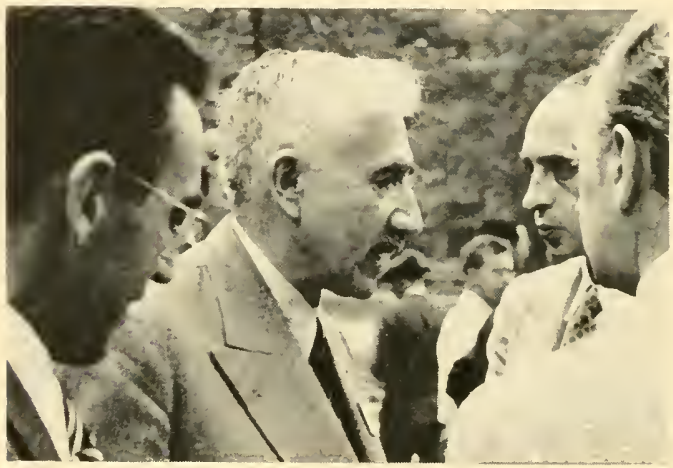

Meyerhof and Grovp

At the Fountainhead

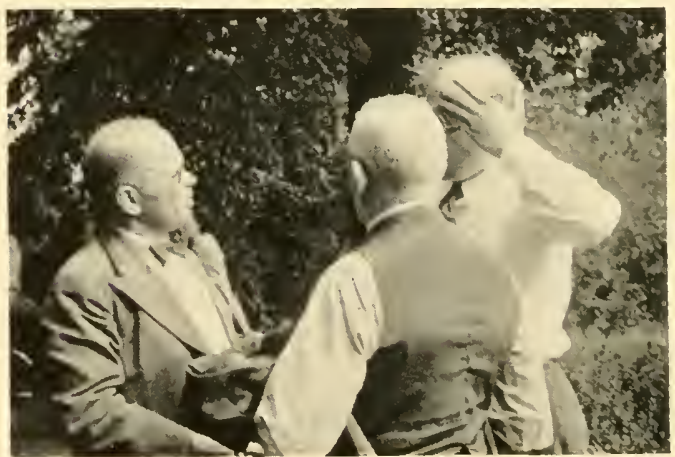

Nord, Neuberg, ant Kletzien

Was it the weather or the argument? BETIVEEN THE SCIENTIFIC SESSIONS 


\title{
Discussion on Hydrogen Transport \\ VAN R. POTTER \\ University of Wisconsin, Chairman
}

\section{Dr. PotTer:}

This afternoon's discussion has been organized with the idea of studying some of the problems which may be considered as open questions at this time. The first speaker will be Dr. Elliott.

\section{THE POSSIBLE ROLE OF INTERMEDIARY METABOLITES AS HYDROGEN CARRIERS}

\author{
K. A. C. ELLIOTT
}

\section{Institute of the Pennsylvania Hospital}

This subject has not been reviewed at length during this symposium, and it is impossible to cover it fully in a brief discussion. Some familiarity with the subject will therefore be assumed, and only certain outstanding problems will be discussed. (For details and bibliographies see references 1-3.)

The most important theory of intermediary metabolites as hydrogen carriers is that of Szent-Györgyi. It may be represented by the following highly simplified diagram, in which heavy arrows indicate transfers of hydrogen atoms (or electrons) from one substance to the next.

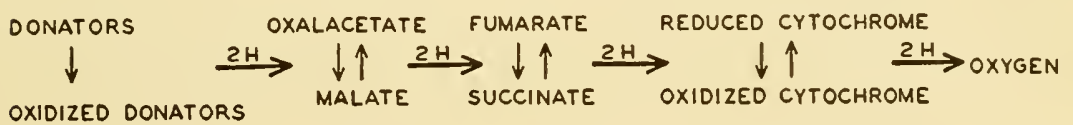

According to this theory, hydrogen from tissue donators reduces oxalacetate to malate; the malate is reoxidized to oxalacetate and the hydrogen is transferred to fumarate, reducing it to succinate; and the succinate is then reoxidized to fumarate by the cytochromecytochrome oxidase system. Malate-oxalacetate and succinatefumarate thus perform functions similar to that of reduced cytochrome-oxidized cytochrome. Known dehydrogenases and cozymase are concerned in the catalysis of the various steps; also, a flavoprotein was believed by the Szent-Györgyi school to mediate in the reduction of fumarate by malate.

Considerable evidence that this mechanism can function in pigeon breast muscle brei has been adduced by the Szent-Györgyi 
school, by Stare and Baumann, and, in connection with work on the citric acid cycle, by Krebs and coworkers, and also by various other workers. Krebs has also shown that at least the succinate-fumarate mediation can occur in B. coli (Escherichia coli). If we assume that the evidence for the Szent-Györgyi mechanism actually proves that it does operate in pigeon breast muscle (see, however, addendum below), the following questions arise:

1. In what tissues may the mechanism be important? Tissues other than pigeon muscle have not been studied exhaustively from this point of view. One of the main tests for the system consists in finding increased or better maintained respiration when small amounts of fumarate or malate, which are interconvertible by tissue fumarase, are added to the tissue brei. Our experience with this test and that of other workers suggests that the system may be important for the respiration of liver, testis, and possibly kidney, but is not very active in brain or skeletal muscle. However, individual reactions of the system, namely, succinate oxidation and oxalacetate reduction, occur rapidly in all these tissues, and Banga, Cori, and coworkers have shown that the presence of fumarate is necessary for the oxidation of pyruvate by kidney and brain dispersions. Banga showed that it was not easy to remove all the four-carbon acids from tissue. It is therefore possible that when added fourcarbon dicarboxylic acids have little effect on the respiration of tissues, these substances may already be present in the tissues in such amounts that their concentration is not a limiting factor of the respiration rate.

The volume of respiration passing through the system would be limited by the activity of the relevant enzymes. Dr. Greig and I found that there was sufficient cytochrome-cytochrome oxidase and succinic dehydrogenase activity in many normal tissues to account for all the respiration through succinate-fumarate, but the succinic dehydrogenase activity was quite low in chick embryo, rat thymus, spleen, pancreas, and some tumors. Breusch found that the rates of oxalacetate reduction were high in muscle, liver, and kidney and moderate in brain and pancreas, but negligible in spleen, lung, placenta, peripheral nerves, and certain rat tumors; the rate in embryo was found to be low by Blasźo.

2. Does the four-carbon dicarboxylic acid system always operate in the same way? Szent-Györgyi himself pointed out that not all substances should be expected to utilize the whole system. Lactate, for instance, might be oxidized through fumarate-succinate but 
probably not through oxalacetate-malate, since the redox potential of the latter system is close to that of pyruvate-lactate. According to the complete theory, fumarate and malate should behave alike, an equilibrium mixture of the two being rapidly produced by the action of fumarase when either is added. But Dr. Libet and I have found that added malate and fumarate (and citrate) have different effects on the repiration of brain suspensions. Greig and Munro found that fumarate, but not malate, caused a lowered respiratory quotient with ox retina and chick embryo.

3. What metabolites are oxidized through the system? The SzentGyörgyi school indicated that carbohydrate derivatives-triosephosphate, alpha-glycerophosphate, and pyruvate-were oxidized through the four-carbon dicarboxylic acid system by tissue suspensions. But Greville reported that not more than 70 per cent of the respiration brought about by fumarate catalysis in muscle was due to carbohydrate oxidation. With suspensions of liver from fasted rats, my wife and I found low respiratory quotient values for the extra respiration caused by adding malate. Leloir and Munoz found that added four-carbon dicarboxylic acids increased the rate of butyric acid oxidation by liver suspension. Dewan and Green, with isolated enzyme preparations, showed the oxidation of betahydroxybutyrate by fumarate. Annau, and my wife and I, noted

- Dr. Eric Ball, Harvard University: My chief objection to the SzentGyörgyi theory is that the inclusion of the malate-oxalacetate system in the chain of reactions as it is written seems to be pointless. Attention has been called to this fact previously (4-6). If we break the scheme into the separate reactions, this becomes evident. Assuming the substrate to be oxidized by means of diphosphopyridine nucleotide $\left(\mathrm{Py}\left(\mathrm{PO}_{4}\right)_{2}\right)$, we may write the first reaction as follows:

$$
\text { Substrate }+\mathrm{Py}\left(\mathrm{PO}_{4}\right)_{2} \rightarrow \mathrm{H}_{2} \mathrm{Py}\left(\mathrm{PO}_{4}\right)_{2}+\text { Oxidized Substrate }
$$

If the pyridine nucleotide is to act as a cyclic catalyst for this reaction, it must be oxidized. According to the Szent-Györgyi scheme, this reoxidation is brought about by oxalacetate. The reaction may be written:

$$
\text { Oxalacetate }+\mathrm{H}_{2} \mathrm{Py}\left(\mathrm{PO}_{4}\right)_{2} \rightarrow \mathrm{Py}\left(\mathrm{PO}_{4}\right)_{2}+\text { Malate }
$$

Now if the oxalacetate in turn is to function as a catalyst, it must be regenerated. This requires that malate be oxidized. The oxidation of malate in the body, however, is known to proceed only through the diphosphopyridine nucleotide:

$$
\text { Malate }+\mathrm{Py}\left(\mathrm{PO}_{4}\right)_{2} \rightarrow \mathrm{H}_{2} \mathrm{Py}\left(\mathrm{PO}_{4}\right)_{2}+\text { Oxalacetate }
$$

This equation is, however, the reverse of equation 2. Thus what is produced is reduced pyridine nucleotide, and we are right where we started when we wrote equation 1 . The introduction of the malate-oxalacetate system into this cycle merely leads us into a blind alley. 
that added four-carbon substances removed, or prevented the formation of, acetoacetic acid in liver and kidney brei. Krebs, working with B. coli [Escherichia coli] indicated that fumarate-succinate mediated the oxidation of glucose, malate, lactate, acetate, glycerol, glyceraldehyde, butyrate, pyruvate, acetoacetate, $l(+)$-glutamate and molecular hydrogen. Apparently the oxidation of many types of metabolite can be mediated by four-carbon dicarboxylic acids.

Szent-Györgyi considered that the four-carbon dicarboxylic acids were quite analogous to coenzymes in their catalytic role. But it must be remembered that they behave also as combustible substances, particularly with kidney cortex slices and also to some extent with brei of various tissues, when they are added in excess. The four-carbon dicarboxylic acids are not the only substances that can act both as catalysts and as substrates for respiration. Carrier possibilities have been shown for pyruvate-lactate, as well as other alpha-keto-alpha-hydroxy acids, adrenochrome, and transamination reactions. It has been suggested that in $B$. coli formate-bicarbonate plays a similar role, and in plants catechol derivatives and dihydroxymaleic acid may be important carriers. Perhaps many other substances may act in this way. In fact, carrier functions could be postulated for all reversible oxidation-reduction systems, and we should perhaps think of all oxidizable metabolites as capable of acting as both substrates and carriers in a dynamic oxidation-reduction continuum.

Addendum. *The Szent-Györgyi theory was first advanced at a time when the succinic dehydrogenase system was the only one definitely known to reduce cytochrome. Mediation by succinatefumarate of hydrogen transport between the majority of metabolites and the cytochrome system thus naturally suggested itself. However, the discovery of flavoproteins, which mediate oxidation of the reduced coenzymes, suggests that a flavoprotein catalyst may bring about more direct oxidation of those substrates that are oxidized through the action of coenzyme-determined dehydrogenases. Dr. Hogness has described cytochrome $c$ reductase, a flavoprotein from yeast that causes cytochrome $c$ reduction by dihydrocoenzyme II. It seems likely that similar catalysts for the oxidation of both dihydrocoenzymes I and II may occur in animal tissues. In that case there would seem to be no necessity for mediation by fumarate-succinate. (Another flavoprotein in yeast, fumarate reductase, has been described by Fischer and coworkers. This enzyme causes the reduction

* In the light of private discussions during the symposium, Dr. Elliott has written an addendum to his remarks. $-E d$. 
of fumarate to succinate by certain leuco dyes; it is distinct from succinic dehydrogenase but could perhaps replace the latter enzyme in the Szent-Györgyi scheme).

While it has been proved that the four-carbon dicarboxylic acids and other metabolites may act as carriers, the previous paragraph indicates that we cannot conclude that fumarate-succinate actually does so. Since malonate inhibits succinate oxidation, the observed inhibition of respiration by malonate has been taken as evidence of the carrier function of succinate-fumarate. But the inhibition of respiration by malonate and the promoting effect of added fumarate may be explained as inhibition and promotion of oxidative metabolism through Krebs' cycle.

Dr. Potter has emphasized the above points (6), and he points out that carrier functions for four-carbon dicarboxylic acids and other substances have been assumed on the basis of the following four criteria: 1. The compound is a natural constituent of tissues. 2. It can be reduced by tissues at rates compatible with the actual rate of oxidation of the substrate whose oxidation it is presumed to catalyze. 3 . The reduced compound can be oxidized by the tissue preparation at an adequate rate. 4 . The compound is able to stimulate catalytically the rate of hydrogen transport in the system under investigation. But it appears that a fifth requirement is necessary to prove carrier function, namely, the compound must be directly reduced by one system and directly oxidized by a second system which is not identical with the first. As Dr. Ball and Dr. Potter have pointed out, oxalacetate is reduced by dihydrocoenzyme I and malate is oxidized by coenzyme I, yielding the dihydrocoenzyme. That is to say, hydrogen from the donator metabolite is passed to the coenzyme, producing dihydrocoenzyme, but subsequent reduction of oxalacetate to malate and reoxidation of the latter merely results in producing the dihydrocoenzyme again. Thus no effective transport of hydrogen has occurred and, unless another biological mechanism for the oxidation of malate is discovered, it seems unnecessary to postulate a carrier function for malate-oxalacetate. It is conceivable, however, that the structural relations of enzymes in tissue may render the passage of hydrogen from the donators to fumarate or flavoprotein easier via coenzyme-oxalacetate-malate-coenzyme than directly via coenzyme in one step.

It seems likely that in tissues there is a dynamic equilibrium between the oxidized and reduced forms of numerous metabolites and that flavoprotein-cytochrome-cytochrome oxidase mechanisms continually abstract hydrogen (or electrons) from the system, while 
few, if any, of the metabolites can be singled out as specially concerned in the hydrogen transport. (This view of the mechanism was suggested to me by Dr. Potter.)

\section{REFERENCES}

1. Szent-Györgyi, A., Studies in Biological Oxidation (Leipzig, 1937).

2. Stare, F. J., and BaumanN, C. A., Cold Spring Harbor Symposia on Quantitative Biology, 7, 277 ( 1939).

3. Elliott, K. A. C., Physiol. Rev., 21, 267 (1941).

4. BALL, E. G., Cold Spring Harbor Symposia on Quantitative Biology, 7, 100 (1939).

5. Martius, C., Ergebnisse Enzymforschung, 8, 247 (1939).

6. Potter, Van R., Medicine, 19, 441 (1940).

\section{Dr. Potter:}

The second question to be considered is the role of the carriers in dismutations and coupled oxidoreductions. ${ }^{*}$ Since it is agreed that the various metabolites may take part in coupled oxidoreductions, it becomes of interest to determine how these reactions may be brought about, and what features these "fermentation" reactions have in common with the oxidative mechanisms. Dr. Ball and Dr. Lipmann will open the discussion.

\section{THE ROLE OF THE CARRIERS IN DISMUTATIONS AND COUPLED OXIDOREDUCTIONS}

\section{With Special Reference to the Flavoproteins}

\section{ERIC BaLl, Harvard University:}

The chief carriers that play a role in dismutations or coupled oxidoreductions are the pyridine nucleotides and flavoproteins. Since the participation of the pyridine nucleotides in such reactions is more common, examples of dismutations and of coupled oxidoreductions involving diphosphopyridine nucleotide may be given first.

The classical example of a dismutation is the so-called Cannizzaro reaction, which may be represented by the following equations:

$$
\begin{aligned}
& \mathrm{R} \cdot \mathrm{CHO}+2 e+2 \mathrm{H}^{+} \rightarrow \mathrm{R} \cdot \mathrm{CH}_{2} \mathrm{OH} \\
& \frac{\mathrm{R} \cdot \mathrm{CHO}+\mathrm{H}_{2} \mathrm{O}-2 e-2 \mathrm{H}^{+} \rightarrow \mathrm{R} \cdot \mathrm{COOH}}{2 \mathrm{R} \cdot \mathrm{CHO}+\mathrm{H}_{2} \mathrm{O} \rightarrow \mathrm{R} \cdot \mathrm{CH}_{2} \mathrm{OH}+\mathrm{R} \cdot \mathrm{COOH}}
\end{aligned}
$$

- This term was not included in the original statement of the question. Dr. Barron rose to point out that the term "dismutation" has a very narrow application and that "coupled oxidoreduction" is the more general expression. 
One molecule of aldehyde undergoes an oxidation to the corresponding acid at the expense of another molecule of aldehyde which is reduced to alcohol. An enzyme which catalyzes this type of reaction, called aldehyde mutase, has been found in liver by Dixon and Lutwak-Mann (1). It requires as a coenzyme diphosphopyridine nucleotide, which thus appears to function in the role of carrier of electrons and hydrogen ions from one aldehyde molecule to another. Another example that might be given is the dismutation of triosephosphate.

The role of a carrier in a so-called coupled oxidoreduction differs from that in a dismutation reaction only in that electrons and hydrogen ions are transferred between molecules of two different substances. A well-known example of such a reaction (alcoholic fermentation) may be written as follows if we omit the coupled phosphorylation steps that accompany it:

$$
\mathrm{CH}_{8} \cdot \mathrm{CHO}+2 e+2 \mathrm{H}^{+} \rightarrow \mathrm{CH}_{2} \mathrm{CH}_{2} \mathrm{OH}
$$

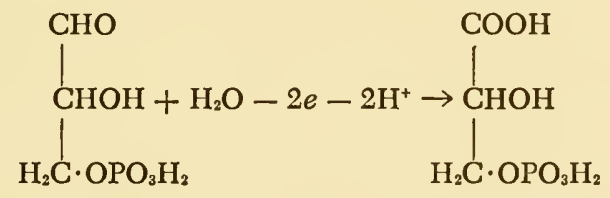

The carrier of the electrons and hydrogen ions between these two aldehyde molecules is also diphosphopyridine nucleotide. The reaction is not classed as a dismutation simply because the aldehyde molecules involved are not identical. One could give other examples of coupled oxidoreductions in which the reacting molecules are more dissimilar and involve diphosphopyridine nucleotide as a carrier.

Such reactions constitute the main type of energy exchange in anaerobic processes. Under aerobic conditions that half of the reaction which involves the loss of electrons and hydrogen ions to the pyridine nucleotide may still occur. The reduced pyridine nucleotide, however, under aerobic conditions, loses its electrons and hydrogen ions to a flavoprotein rather than to another substrate molecule. This type of reaction, then, might be listed as still another class of coupled oxidoreductions.

As compared with the pyridine nucleotides, the flavoproteins participate in only a few direct reactions with substrate molecules. Examples of dismutations or coupled oxidoreduction reactions such as have been given for the pyridine nucleotides are less plentiful for the flavoproteins. One example of a dismutation reaction which may be classed as involving a flavoprotein is that for the substrate 
xanthine. Green (2) has shown that, in the presence of xanthine oxidase, xanthine undergoes the following reactions:

$$
\begin{aligned}
& \text { Xanthine }+2 e+2 \mathrm{H}^{+} \rightarrow \text { Hypoxanthine } \\
& \text { Xanthine }-2 e-2 \mathrm{H}^{+} \rightarrow \text { Uric Acid } \\
& \hline 2 \text { Xanthine } \rightarrow \text { Hypoxanthine }+ \text { Uric Acid }
\end{aligned}
$$

The carrier of the electrons and hydrogen ions between two molecules of xanthine may in this case be due to the flavin portion of xanthine oxidase. Examples of oxidoreduction reactions between two different substrates involving flavoproteins are not known to me. It may be that no such reactions exist, since each flavoprotein appears to be substrate specific. An indirect reaction of a flavoprotein as a carrier between two different substrates is, however, conceivable. This would be the case with two different substrates whose direct reaction occurred only with each of the pyridine nucleotides, as shown by the following equations:

\begin{tabular}{l} 
Oxidized Substrate $A+\mathrm{H}_{2} \mathrm{Py}\left(\mathrm{PO}_{4}\right)_{2} \rightarrow \mathrm{Py}\left(\mathrm{PO}_{4}\right)_{2}+$ Substrate $A$ \\
\hline $\mathrm{H}_{2} \mathrm{Py}\left(\mathrm{PO}_{4}\right)_{2}-2 e-2 \mathrm{H}^{+} \rightarrow \mathrm{Py}\left(\mathrm{PO}_{4}\right)_{2}$ \\
$\mathrm{Py}\left(\mathrm{PO}_{4}\right)_{3}+2 e+2 \mathrm{H}^{+} \rightarrow \mathrm{H}_{2} \mathrm{Py}\left(\mathrm{PO}_{4}\right)_{3}$
\end{tabular}

Here in order for oxidized substrate $A$ to react with substrate $B$, the reduced triphosphopyridine nucleotide must react with diphosphopyridine nucleotide. Whether such a direct reaction is possible is, I believe, unknown. It is possible, however, that a flavoprotein might act as a carrier of electrons and hydrogen ions between the two pyridine nucleotides. Such a reaction could thus be classed as a coupled oxidoreduction involving a flavoprotein.

Fritz Lipmann, Massachusetts General Hospital:

Flavoproteins are at present known to react in two ways: (1) with oxygen directly, i.e., as mediators between substrates and oxygen; (2) as mediators between pyridine enzymes and other catalysts or oxygen.

The second function has been thoroughly studied in reactions representing anaerobic parts of essentially aerobic reaction chains: the bridging between pyridine nucleotide and methylene blue (Haas, Straub, Green) and between pyridine nucleotide and cytochrome (Haas, Horecker, and Hogness). Although it would be suspected that a flavin mediator is needed in a great variety of purely anaerobic reactions, especially in those between a pyridine and a non- 
pyridine enzyme, not much is definitely known about such action of flavoproteins. One example, however, is the dismutation of pyruvic acid (Lipmann), in which a flavin component was shown to participate in a coupled oxidoreduction. Here the flavin mediates between two enzyme systems of different types, i.e., between lactic and pyruvic dehydrogenase:

$$
\begin{aligned}
& \text { pyruvate } \rightarrow \text { lactate } \\
& 2 \mathrm{H} \uparrow(\text { flavin) } \\
& \text { pyruvate..... phosphate } \rightarrow \text { acetylphosphate }+\mathrm{CO}_{2}
\end{aligned}
$$

The presence of a flavoprotein in yeast, which catalyzes the reduction of fumarate (Fischer), suggests the mediator function of such a flavoprotein between pyridine enzymes and fumarate. For example, in the dismutation of fumaric acid (Green) electron transfer presumably occurs between malic dehydrogenase, a pyridine enzyme, and succinic dehydrogenase, a non-pyridine enzyme:

$$
\begin{aligned}
& \text { fumarate } \rightarrow \text { succinate } \\
& 2 \mathrm{H} \uparrow \text { (flavin) }
\end{aligned}
$$

fumarate $\rightleftarrows$ malate $\rightarrow$ oxalacetate

It is probably accidental that in both examples for anaerobic flavin mediation, the coupled oxidoreduction is a so-called dismutation. This might serve to show that in most enzymatic dismutations the underlying reaction is, in fact, an oxidoreduction between two enzyme systems catalyzing two fundamentally different reactions. But in dismutation the metabolic substrates for both enzyme systems derive from the same compound. In the cases discussed, the oxidant in the oxidation-reduction reaction is the compound proper, pyruvate or fumarate, and the reductant is a transformation product of the added compound. Oxidant and reductant belong, respectively, to two different oxidation-reduction systems with widely different oxidation-reduction potentials. In dismutation (reaction 1) the lactate-pyruvate system of $\mathrm{E}_{0}{ }^{\prime}-0.18$ volts reacts with the pyruvate... $\mathrm{HX}$-acetate $+\mathrm{CO}_{2}$ system of $\mathrm{E}_{0}^{\prime}$ below -0.4 volts. In the second reaction the succinate-fumarate system of $\mathrm{E}_{0}{ }^{\prime} 0.0$ volts reacts with the malate-oxalacetate system of $\mathrm{E}_{0}{ }^{\prime}-0.17$ volts. These large energy differences between the reacting oxidation-reduction systems explain why dismutation occurs.

\section{REFERENCES}

1. Dixon, Malcolm, and Lutwak-Mann, Cecilia, Biochem. J., 31, 1347 (1937).

2. Green, David Ezra, Biochem. J., 28, 1559 (1934). 


\section{THE PHYSICO-CHEMICAL MECHANISM OF HYDROGEN TRANSPORT}

\section{Kurt Stern, Yale University:}

Dr. Ball, on Thursday morning, spoke of a gap between the cytochrome oxidase-cytochrome system and the substrate-dehydrogenase systems. It appears that this gap may now be considered filled by several flavoproteins, e.g., Hogness and Haas's cytochrome reductase, Euler and Green's diaphorase (coenzyme factor), or Szent-Györgyi's dicarboxylic acid system, etc.

It is incorrect to label the entire process of cell respiration one of hydrogen transport, since the first stages, from oxygen through the four iron atoms of the respiratory ferment and the three cytochromes, are concerned exclusively with electron transfers. This brings up a difficulty in the formulation of the elementary steps that connect the iron systems with the dehydrogenase systems. The oxido-reductive changes taking place in the former are one-electron transfers; the dehydrogenation reactions, on the other hand, are formulated as bivalent processes, involving the loss and uptake of two hydrogen atoms per molecule. The most satisfactory way of resolving this dilemma without invoking the existence of highly problematical trimolecular reactions is to assume, with Michaelis, that the apparently bivalent dehydrogenations are actually two-step processes, involving the transfer of one hydrogen atom or its equivalent at a time, with the intermediate formation of semiquinoid radicals.

It seems to be no mere coincidence that the macromolecular preparations, called cytochrome oxidase by Keilin, contain a number of components: the oxidase, cytochromes $a$ and $b$, succinic dehydrogenase, etc., which are all concerned with what Oppenheimer calls the "terminal oxidation" of metabolites. It is reasonable to assume that these particles represent functional units which contain these catalysts in a spatial arrangement which facilitates the progress of this important phase of cell respiration in a constant pattern and at a constant and high rate.

\section{Dr. Potter:}

The dilemma of which Dr. Stern has spoken, that is, the mechanism for getting a bivalent dehydrogenation system to react with a one-electron system, may possibly be resolved by the formation of a complex made up of the proper components of the hydrogen 
(electron) transport system. I believe Mr. Haas has some experimental data which have considerable bearing on this question.

EnwIN HaAs, University of Chicago:

For a long time the mechanism of respiration was studied only by considering intermediary metabolites. A more direct approach to that problem is now possible, since some of the respiratory enzymes have become available in isolated form and since their functional groups are known.

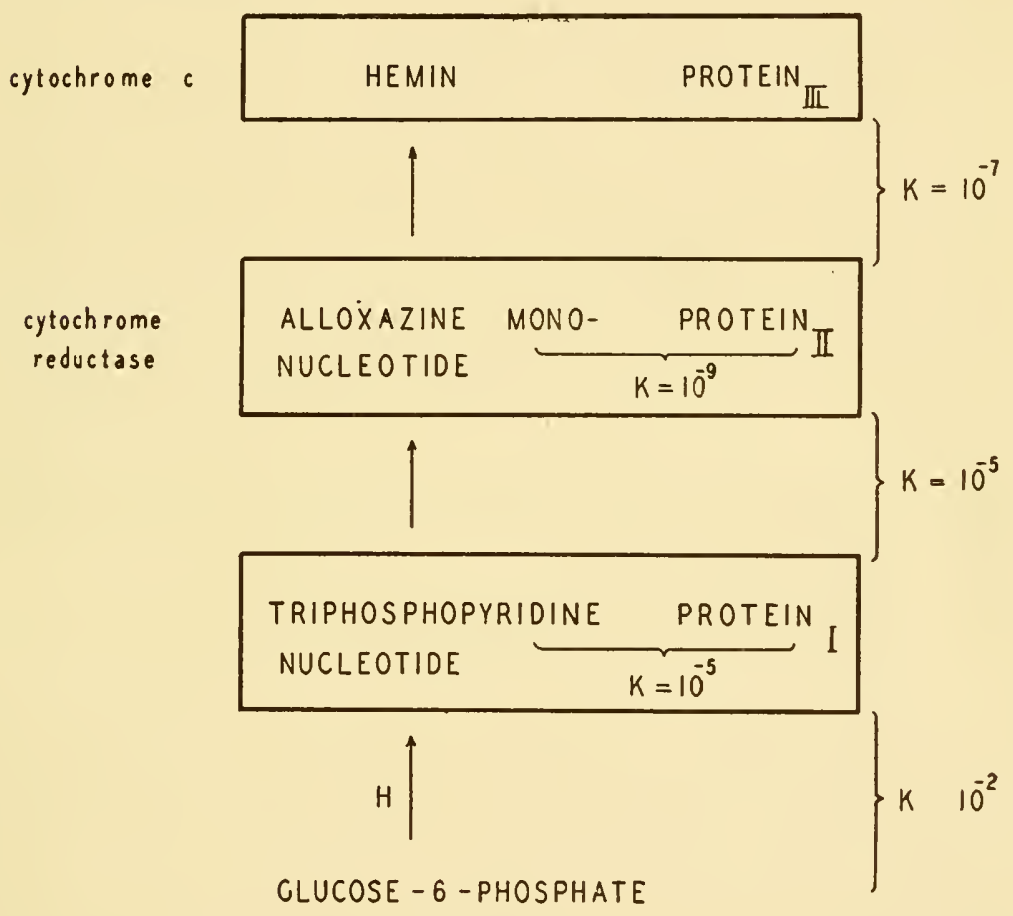

FIGURE 1

Figure 1 illustrates a part of the respiratory system which brings about the reduction of cytochrome $c$ by glucose-6-phosphate. The components of the system are arranged in the order in which they react; the details concerning their chemical structure are omitted. Hydrogen or electrons from the glucose are passed on to the pyridine nucleotide, thence to the alloxazine mononucleotide, and then to the iron atom in cytochrome $c$. In each step a specific protein must be present which, together with the prosthetic group, forms the active 
enzyme. By selecting proper concentrations any reaction in this scheme can be made the limiting factor. Thus by measuring the rate with which cytochrome $c$ is reduced, it is possible to determine rates of reaction and to demonstrate formation of enzyme complexes for any of the reactions involved. Negelein and Haas have shown by ultraviolet spectroscopy the formation of a complex between protein I, triphosphopyridine nucleotide, and glucose-6-phosphate. With the method indicated above the dissociation of the cytochrome reductase into alloxazine mononucleotide and protein II can be demonstrated if to a small but constant amount of protein II increasing amounts of alloxazine mononucleotide are added.

The dissociation constant of cytochrome reductase is small $\left(\mathrm{K}=1 \times 10^{-9} \mathrm{M}\right)$, and under physiological conditions this enzyme will therefore be present as the practically undissociated complex. The value of the spectrophotometric method for the study of enzyme reactions is well demonstrated here, since $10^{-6} \mathrm{mg}$. of flavin are sufficient for accurate determinations. Not only the relation between the prosthetic group and protein of one enzyme may be studied with this system, but also the interaction of two different enzymes. For example, we can measure the rate of reaction when increasing amounts of dihydrotriphosphopyridine nucleotide are added to a constant amount of cytochrome reductase. The velocity of the reduction of the enzyme is given by

$$
\frac{\mathrm{d}(\mathrm{CR})}{\mathrm{dt}}=\frac{k_{r}(\mathrm{~T})(\mathrm{CR})}{(\mathrm{T})+K_{D}}
$$

in which ( $\mathrm{T}$ ) and (CR) are the concentrations of triphosphopyridine nucleotide and cytochrome reductase, respectively, $k_{r}$ is the firstorder velocity constant, and $K_{D}$ the dissociation constant of the pyridine-alloxazine complex. Rate constants and dissociation constants have been determined at different temperatures, and by applying the Arrhenius equation the energy of activation is found to be about $10 \mathrm{~kg}$. cal., and the heat of dissociation about $2 \mathrm{~kg}$. cal., for the reaction in which cytochrome reductase is reduced by dihydrotriphosphopyridine nucleotide.

Similar experiments have been made for the purpose of studying the oxidation of cytochrome reductase by cytochrome, and again the formation of a complex between the two reaction partners could be established. Furthermore, from the results of the kinetic determinations it can be concluded that in the course of the oxidation of alloxazine free radicals are involved. This could almost be antici- 
pated, for the alloxazine undergoes a valence change of two, whereas cytochrome undergoes a valence change of one, as Dr. Stern has just pointed out.

To summarize, the facts concerning this part of the respiratory system are, then, as follows: The protein of the cytochrome reductase is bound simultaneously to alloxazine mononucleotide, to triphosphopyridine nucleotide, and to cytochrome. Approximate values for the different dissociation constants are given in Figure 1. The energy of activation is low, about $10 \mathrm{~kg}$. cal. One may venture to say that the binding forces are of the Van der Waal type rather than ordinary bond forces, which would involve much higher bond energies.

The task of the protein may be (a) to establish the proper geometrical configuration between the different prosthetic groups; and (b) to aid in the formation and stabilization of free radicals. The first point may be offered as a working hypothesis to explain the specificity of the proteins, and the second point may explain the tremendous activity of this catalyst. These experiments were done in Professor Hogness' laboratory, with Drs. B. L. Horecker and C. J. Harrer.

\section{Mr. HaAs:}

The formation of complexes in the course of these enzymatic reactions can be demonstrated in two independent ways.

1. Kinetic Measurements.-In agreement with equation 1, the velocity of the reaction is proportional not to (T) or to (CR), as one would expect from an ordinary bimolecular reaction, but to the amount of enzyme present in the form of the complex.

2. Spectroscopic Measurements.-Alloxazine mononucleotide has its maximum absorption at wave length $445 \mathrm{~m} \mu$; the addition of the protein of the old yellow enzyme causes the maximum absorption to migrate to wave length $465 \mathrm{~m} \mu$, and the further addition of triphosphopyridine nucleotide to wave length $475 \mathrm{~m} \mu$. Thus the free flavin, the flavin-protein complex, and the flavin-protein-pyridine complex can easily be distinguished by their color.

\section{Dr. POTTER:}

Thus far the emphasis has been on the cytochrome system as one of the links in the hydrogen transport system. Yet there is an accumulating body of evidence which indicates that an alternate path

- This statement of Mr. Haas' was made in reply to a question from the floor regarding the proof of formation of an enzyme-substrate complex. $-E d$. 
of hydrogen transport may exist. Dr. Stotz has agreed to open the discussion of this interesting possibility.

\title{
POSSIBILITY OF A BY-PASS AROUND THE CYTOCHROME SYSTEM
}

\author{
ELMER STOTZ \\ Harvard University
}

The possibility of a "by-pass" around the cytochrome system in certain phases of tissue respiration has elicited considerable discussion of late. The discovery of the flavoproteins, and their function in isolated systems as autoxidizable substances, had raised a doubt as to the exclusive role of the iron system in "oxygen activation." However, this threat to the autonomy of the iron compounds was dispelled by the correction of certain technical points and by the work of Theorell and of Barron. The most recent threats have come from a study of the effects of cyanide and azide (as oxidase inhibitors) on the respiration of "resting" and "stimulated" tissues. The work of Stannard and of Korr on this topic is reviewed in my paper on page 169. Their results may be summarized briefly by the statement that the respiration of "resting" tissue is insensitive to azide, whereas that of the stimulated tissue becomes azide-sensitive. Tissues in either phase may, however, be cyanide-sensitive. The "by-pass" theory therefore implies that in the resting tissue an oxidation pathway other than the cytochrome system is functioning. In Korr's terminology, tissues that have been stimulated may liberate substances that "link" or "gear" the reducing systems to the cytochrome system.

The by-pass theory, although possibly correct, is in my judgment a rather sweeping conclusion to make from evidence based chiefly on the use of these inhibitors, and without knowledge of the nature of an alternative pathway. Even if we assume an identical action of the inhibitors in isolated systems, as in the muscle and tissue slices, we are far from understanding the mechanism of their action. Stannard believes that cyanide affects not only cytochrome oxidase but also other enzymes essential in respiration, and, to be sure, he offers some evidence for this belief. On the other hand, this does not imply that azide is any more specific than cyanide and that only the effects of the former need be considered.

It would seem more conservative at the moment to consider how the differences between cyanide and azide might be explained on the basis of the existing knowledge of the cytochrome-cytochrome 
oxidase system and of the action of nitrogen compounds on iron systems.

The relations of cytochrome oxidase and cytochrome $c$ are such in the oxidation of hydroquinone, for instance, that a decrease of oxidase, which would ordinarily cause a decreased hydroquinone oxidation, could be compensated for by an increase in reduced cytochrome. It has been shown that cyanide does in effect "remove" a certain portion of the oxidase. Hence in a resting tissue, where most of the cytochrome is in the oxidized state, a decreased oxidase could be compensated for by increased reduction of the cytochrome. In the more active state of metabolism, where there is a small reserve of oxidized cytochrome, such compensation is less possible. The more undissociated the oxidase-inhibitor complex the less efficient would be the compensation. Since azide is a "less powerful" oxidase inhibitor than cyanide, and since the greatest sensitivity to azide is found in the "active" state, these factors may be operative.

Dr. Ball has considered at some length, in this symposium, the possible differences between cyanide and azide as nitrogenous compounds uniting with the oxidase $(\mathrm{Fe})$ and the lowering of potential caused thereby. He has pictured how a difference in the ability of the two compounds to unite with the oxidized and reduced forms of the oxidase could lead to differences in the effective potential of the complex formed. Thus the union with cyanide could lead to an oxidase complex with a potential lower than that of cytochrome $c$, hence possessing a low catalytic power. As a result of combination of higher concentrations of azide with both oxidized and reduced oxidase, an effective potential might be reached which could be somewhat higher than that of cytochrome $c$. Although such a complex would be less efficient than the original oxidase, it might nevertheless be sufficient for the low metabolism that exists in the resting state, although insufficient for the metabolism of the "active" tissue. This theory was not advanced to overthrow the "by-pass" theory, but only to call attention to other possible explanations for the differences between azide and cyanide. Perhaps these considerations should be exploited before postulating, through the mechanism of as yet unknown enzymes, a "by-pass" around the cytochrome system in respiration. 


\title{
Pasteur Effect
}

\author{
FRITZ LIPMANN \\ Massachusetts General Hospital
}

$W_{\text {may be classified into (1) strict aerobes, equipped only with }}^{\text {ITH RESECT to their dependence on oxygen supply organisms }}$ respiratory metabolic systems, (2) strict anaerobes, equipped only with anaerobic fermentative metabolic systems, and (3) facultative organisms, equipped with both respiratory and fermentative systems. This commonly used classification should not be followed too rigidly, however, for intermediate states between the main classes are common in nature, and adaptive interconversion has been widely observed.

The organisms in each of the first two groups rely exclusively on one form of energy supply, respiratory or fermentative, respectively. The third group, however, has developed the two mechanisms side by side. It is with this latter group that we shall deal, and more specifically with the interrelation between their respiratory and fermentative mechanisms.

Most doubly equipped organisms possess in the Pasteur effect a regulatory device that enables them to use, as occasion demands, either their aerobic or their anaerobic systems. By the operation of this effect their fermentative apparatus is blocked in the presence of sufficient oxygen, and energy is furnished almost exclusively by the far more efficient and powerful respiratory apparatus. When oxygen is lacking, however, the fermentation system is brought into operation.

The following example may serve to illustrate the energetic structure of a facultative anaerobic organism. A power plant uses as a source of energy cheap water power; this may be compared to the "cheap" respiratory energy. But because of seasonal variations of flow the water power may not be entirely reliable and hence as a safeguard against a deficiency in the supply of power a more expensively operating steam engine is built into the plant; this may be compared to "expensive" fermentation. For obvious reasons the plant will be equipped with a switch mechanism-its "Pasteur effect" -which keeps the steam engine from functioning so long as the water flow supplies sufficient energy but throws it into operation when water power is lacking. 
An impressive physiological example of a mechanism utilizing both respiratory and fermentative energy supply is the muscle. Figures 1 and 2, after experiments by Bang (1), reproduce measurements of oxygen consumption and lactic acid formation (blood lactate) on human beings during physical work. During a prolonged period of not too hard work (Figure 1) blood lactate at first increases moderately but returns, during the first quarter of the period, almost to the resting level. An anaerobic energy supply is observed only at the beginning, when an adequate oxygen supply is lacking, and until respiration climbs to the equilibrium level. For excessive short-term work the picture is different, as shown in Figure 2. An excessive and long-continued increase of blood lactate signifies a large expenditure of anaerobic energy. In such a situation the adaptation is much too

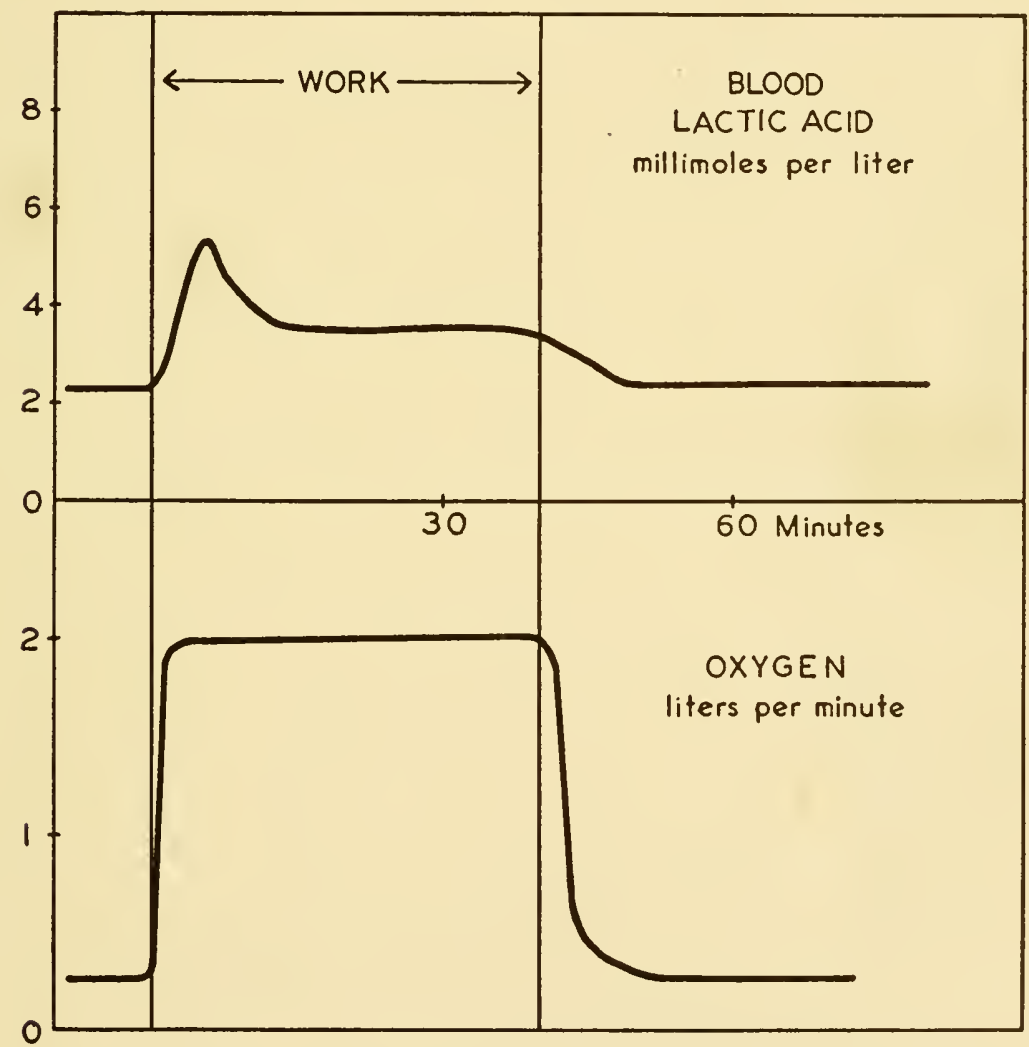

Figure 1.-Oxygen consumption and blood lactate with moderate work. The slight initial rise of lactate output coincides with the period of adaptation, before the oxygen consumption rises to the equilibrium level. (After O. Bang, ref. 1.) 
slow to supply oxygen in time, and the muscle has to rely almost entirely on the anaerobic energy of glycolysis.

The Efficiency of Aerobic and Anaerobic Metabolism

Fermentations are energy-yielding rearrangements of the atoms constituting the glucose molecule. These are oxidation-reduction reactions in which, after cleavage, one part of the molecule is

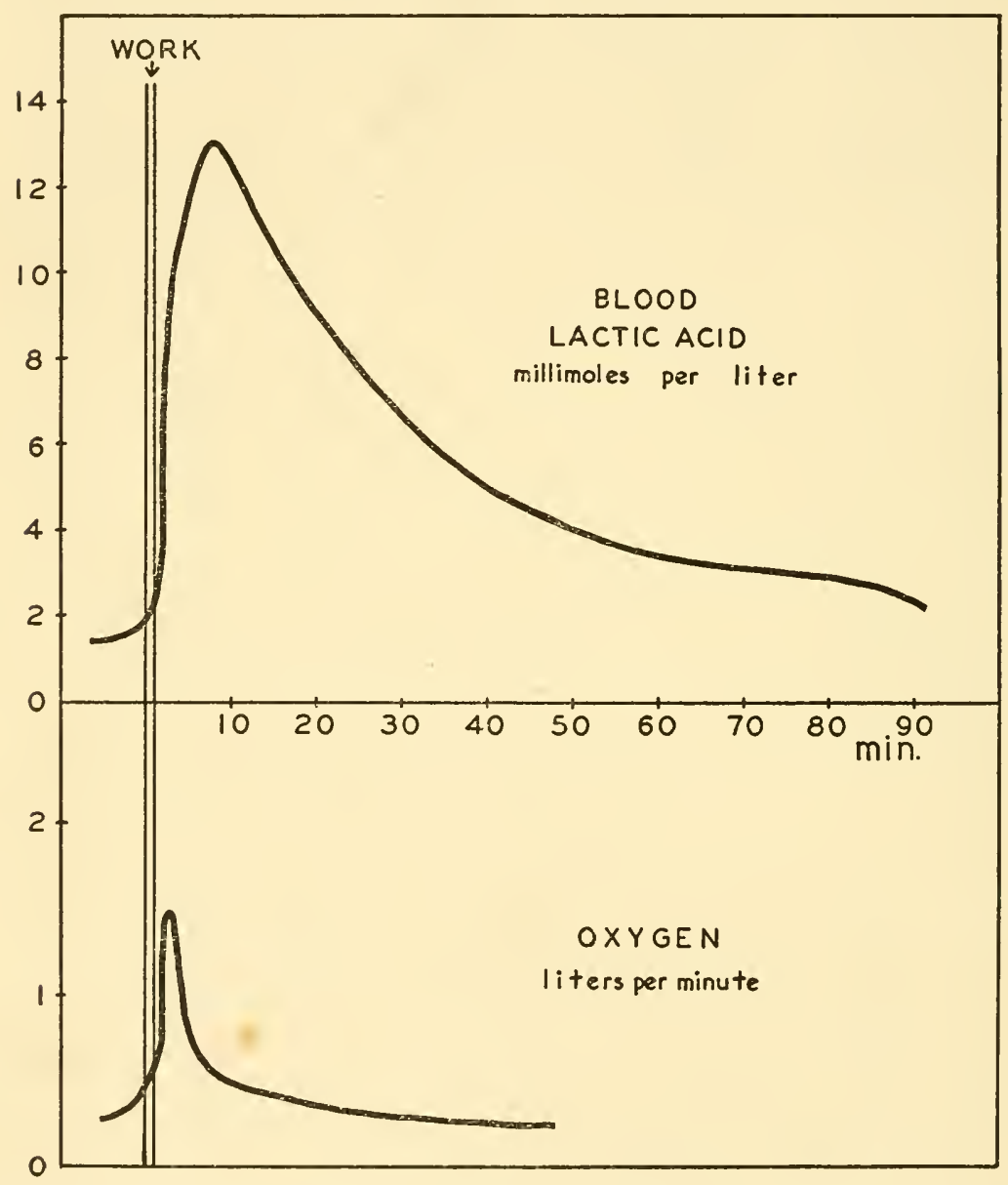

Figure 2.- Oxygen consumption and blood lactate with strenuous work. The excessive lactate formation signifies predominantly anaerobic energy supply. A moderate rise of oxygen consumption occurs first after completion of the work in the period of restitution. The slow fall of lactate in the blood indicates a relatively slow removal of lactate by resynthesis or oxidation. (After O. Bang, ref. 1.) 
oxidized at the expense of the other part, which accordingly is reduced. Energetically probably the most efficient reaction is one such as the propionic acid fermentation: $3 \mathrm{C}_{6} \mathrm{H}_{12} \mathrm{O}_{6}=4 \mathrm{C}_{2} \mathrm{H}_{5} \mathrm{COOH}+$ $2 \mathrm{CH}_{3} \mathrm{COOH}+2 \mathrm{CO}_{2}+2 \mathrm{H}_{2} \mathrm{O}$. The energetic yield is $61 \mathrm{~kg}$.-cal. of heat per mole of glucose. According to Burk (2), the change in free energy is approximately $18 \mathrm{~kg}$.-cal. higher per mole of glucose than the heat exchange. The probable maximum for a fermentative breakdown of carbohydrate thus amounts to $79 \mathrm{~kg}$.-cal. per mole. This is 11.5 per cent of the $686 \mathrm{~kg}$.-cal. to be obtained by respiratory breakdown. The more common lactic acid and alcoholic fermentations do not reach this maximum but yield only $54 \mathrm{~kg}$.-cal. (36 kg.-cal. plus $18 \mathrm{~kg}$-cal. entropy change), or 7.9 per cent of the heat of combustion.

These values represent the theoretical maximum for fermentation and respiration. To compare the efficiencies of the two reactions in the cell we must know how much of the energy of each reaction is actually available to the cell. In a recent paper (3) the author has pointed out that from 40 to 70 per cent of the theoretical fermentation energy is utilizable. This is deduced from the fact that in the muscle up to 40 of the $54 \mathrm{~kg}$.-cal. derived from glycolysis can be stored as four energy-rich phosphate bonds in phosphagen, the energy of which is utilizable for muscular work and other purposes.

Until fairly recently the view was favored that respiration energy was much less utilizable than fermentation energy-in other words, that fermentation energy was relatively more valuable than would be indicated by a comparison of theoretical caloric yields. Recent results, however, for the conversion of oxidation into phosphate bond-energy strongly indicate that such is not the case. With oxidation of pyruvic acid in the brain, Ochoa (4) found that for each molecule of oxygen consumed four energy-rich phosphate bonds were generated. With carbohydrate oxidation in heart muscle, according to Belitzer and Tzibakova (5) as many as seven energy-rich phosphate bonds (for nomenclature of. ref. 3) might be formed per molecule of oxygen. One energy-rich phosphate bond represents from 10 to $12 \mathrm{~kg}$.-cal. of utilizable energy. The six moles of oxygen oxidizing one mole of carbohydrate could therefore generate from $11 \times(4$ to 7$) \times 6=260$ to $460 \mathrm{~kg}$.-cal. of utilizable energy, or 40 to 68 per cent of the theoretical.

The unexpectedly high yield obtained in these recent experiments shows that there is probably no great difference in utilizability between fermentation and respiration. Therefore it seems permissible 
to take the theoretical caloric value of 54 and $686 \mathrm{~kg}$.-cal., respectively, as a basis for comparing their efficiencies, and to conclude that only one-twelfth to one-ninth of the total possible energy is made available to the cell by the anaerobic fermentation of the glucose molecule.

From this calculation the superior economy of respiratory metabolism becomes evident. To draw the same amount of energy from fermentation as from respiration the cell must use from nine to twelve times as much substrate. In reality the anaerobic energy is rarely equal to the aerobic. As a rule a fully developed facultative anaerobe uses anaerobically only four to eight times as much substrate as aerobically, thus reaching on the average half the energy level of the aerobic state. From these considerations the economical and regulatory aspect of the Pasteur effect becomes evident. Through its operation the voluminous fermentative metabolism is allowed to proceed only in anaerobiosis, as is indicated in the following scheme, which represents the increase in glucose utilization following change from aerobic to anaerobic conditions:

OXYGEN

$$
\left(\mathrm{Q}_{\mathrm{O}_{2}}=6 ; \mathrm{Q}_{\mathrm{G}}^{\mathrm{o}_{2}}=0\right)
$$

1. Glucose $+6 \mathrm{O}_{2} \rightarrow 6 \mathrm{CO}_{2}+6 \mathrm{H}_{2} \mathrm{O}$
NITROGEN

$\left(Q_{G}^{N_{2}}=10\right)$

1. Glucose $\rightarrow 2$ Lactate

2. Glucose $\rightarrow 2$ Lactate

3. Glucose $\rightarrow 2$ Lactate

4. Glucose $\rightarrow 2$ Lactate

5. Glucose $\rightarrow 2$ Lactate

Some examples of "ideal" facultative anaerobic cells are given in Table 1. From the $Q$ values the corresponding glucose consumption and the caloric yields are calculated. One cubic millimeter of respiration oxygen corresponds to the utilization of 1.34 micrograms of glucose and the yield of $5.2 \times 10^{-3} \mathrm{cal}$; one cubic millimeter of fermentation carbon dioxide corresponds to the utilization of 4.03 micrograms of glucose and the yield of $1.2 \times 10^{-3} \mathrm{cal}$.

In the cases cited the large fermentative metabolism disappears completely in aerobiosis. In the experiment with the fish retina almost three-fourths of the aerobic energy is made available through anaerobic metabolism and correspondingly a three- to sevenfold anaerobic increase of glucose consumption occurs.

\section{The Metabolic Structure of Cells}

In the middle of the metabolic type scale are placed the organisms that alternate between anaerobic and aerobic metabolism of 
similar efficiency. In the upper part are predominantly aerobic types, e.g., kidney and liver and many plant cells with relatively small fermentative capacity, and at the top of the scale is a strict aerobe, azotobacter, with $\mathrm{Q}_{2}$ of 2000 and no trace of fermentation.

Table 1.-Effect of oxygen on glycolysis

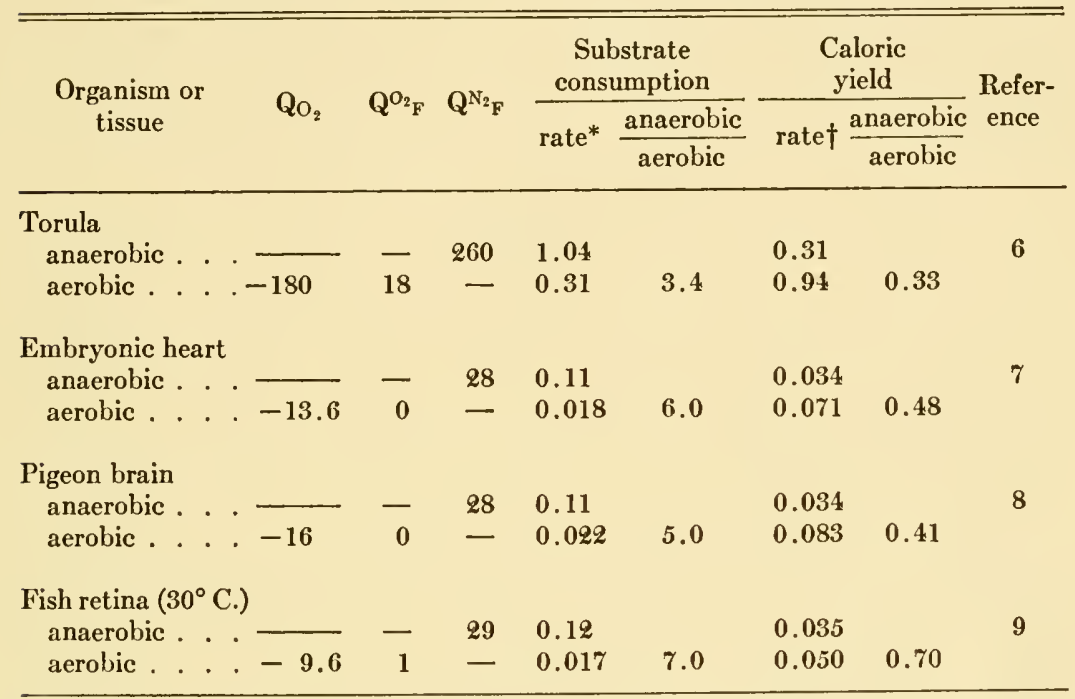

* mg. glucose per mg. dry weight per hour. † calories per mg. dry weight per hour.

On the anaerobic side, below the middle, are a variety of types representing gradations down to exclusively anaerobic life. Here fermentation is partly or wholly persistent in the presence of oxygen, and respiration becomes a more or less residual function.

\section{BACTERIA}

The anaerobic type of life is most common among the bacteria, but it occurs frequently in the animal kingdom, especially among invertebrates. In all stages of phylogenetic development, life either chooses or is forced to adapt to anaerobic conditions, and similar metabolic arrangements correspond to similar environmental conditions. Transition from alternative to exclusive anaerobiosis is well illustrated by a tabulation of the metabolism of the common yeasts (Table 2), taken from Meyerhof's classical paper (6).

The almost exclusively anaerobic cultured yeasts used in the manufacture of alcoholic beverages presumably developed from the 
alternating torula or wild yeast type. Baker's yeast is intermediate, having a fair respiration and partially persistent aerobic fermentation. The metabolic type is not rigidly fixed. With aeration there is adaptation to the respiratory type, and with the exclusion of air the reverse is easily achieved (Table 2). It may be noted that the reappearance of respiration is accompanied by aerobic repression of fermentation. The history of the manufacture of baker's yeast is an impressive illustration of the economic superiority of aerobic metabolism (10). The earlier "Vienna" procedure of growing yeast

Table 2.-Yeast metabolism

\begin{tabular}{|c|c|c|c|c|c|}
\hline Type & $\mathrm{QO}_{2}$ & $\mathrm{Q}^{\mathrm{O}_{2}} \mathrm{~F}$ & $\mathrm{Q}^{\mathrm{N}_{2}} \mathrm{~F}$ & $\frac{\left(\mathrm{Q}^{\mathrm{N}_{2}} \mathrm{~F}-\mathrm{Q}^{\mathrm{O}_{2}} \mathrm{~F}\right) \times 3}{\mathrm{QO}_{2}}$ & $\begin{array}{l}\text { Inhibition } \\
\text { per cent }\end{array}$ \\
\hline Wild yeast. . . . . . . & -180 & 18 & 260 & 4 & 93 \\
\hline Baker's yeast . . . . . & -87 & 95 & 274 & 6.2 & 65 \\
\hline Brewer's yeast . . . . . & -8 & 213 & 233 & 7.5 & 8 \\
\hline Same after 15 hours aeration & -73 & 113 & 193 & 3.3 & 42 \\
\hline
\end{tabular}

without agitation has now been almost entirely replaced by the aeration procedure, for it has been found that through aeration the yield can be greatly increased with the same amount of culture fluid. In metabolic terms, the same amount of metabolized substrate yields a larger amount of yeast material with economical respiration than with uneconomical fermentation.

As a measure of the Pasteur effect two differently derived units are recorded in the last two columns of Table 2. In the first of the two the Meyerhof Oxidation Quotient is calculated. This relates the disappearance of fermentation to the magnitude of respiration. When three times the difference between fermentation in nitrogen and fermentation in oxygen is divided by the respiration in oxygen, the quotient represents the relation between the glucose equivalent of fermentation and that of respiration. Disregarding underlying theoretical implications, it states how much fermentation glucose is replaced by oxidized glucose when respiration is allowed to occur. Stressing the economical significance of the quotient, we have proposed to call it a replacement quotient (3). In the next column the percentage of inhibition is calculated. From the recorded figures one would suspect a relationship of some kind between the magnitude of respiration and the Pasteur effect. This observation originally led Meyerhof to a universal application of his resynthesis theory as an explanation of the Pasteur effect. 
Although a broader discussion of the theory of the Pasteur effect is reserved for a later paragraph, some interesting experiments relating to the constancy of the Meyerhof Quotient may be mentioned here. These experiments on the effect on the oxidation quotient of the Z-factor of v. Euler, a factor stimulating only fermentation, are taken from a paper by Meyerhof and Iwasaki (11). See Table 3.

For the same yeast, $Q$ values, the oxidation quotient, and the percentage of inhibition are listed with and without the stimulating

Table 3.-Influence of medium on fermentation and oxidation quotient

(Reference 11)

\begin{tabular}{lllrrrr}
\hline \hline Yeast & \multicolumn{1}{c}{ Medium } & $\mathrm{QO}_{2}$ & $\mathrm{Q}^{\mathrm{O}_{2} F}$ & $\mathrm{Q}^{\mathrm{N}_{2} F}$ & O.Q.* & $\begin{array}{c}\text { Inhibition } \\
\text { per cent }\end{array}$ \\
\hline Baker's yeast I & glucose in phosphate & -37.5 & 44.5 & 119 & 6 & 62 \\
& plus wort & -37 & 96 & 212 & 9.4 & 55 \\
Baker's yeast II & glucose in phosphate & -40.5 & 105 & 199 & 7 & 47 \\
& plus molasses & -45.6 & 228 & 374 & 9.6 & 39 \\
Strain XII & sugar in phosphate & -10 & 49 & 57 & 2.3 & 14 \\
& plus yeast extract & -10.5 & 137 & 167 & 8.5 & 18 \\
\hline
\end{tabular}

* Meyerhof oxidation quotient.

factor. Here with the same respiration but variations in anaerobic and aerobic fermentation, the relative influence of respiration on the disappearance of fermentation increases, whereas the percentage of inhibition remains constant. These experiments are more consistent with an explanation of the Pasteur effect as an inhibition of some kind resulting from the presence of oxygen but independent of the magnitude of respiration. Similar results were recently reported by Burk, Winzler, and du Vigneaud (12), who studied the metabolism of a biotin-deficient yeast. They found oxidation quotients up to 20 , whereas 12 is the theoretical maximum for a resynthesis theory.

The results of these experiments are discussed in some detail in order to draw attention to two lines of approach to an explanation of the phenomenon. The first set of data, showing parallel rates of respiration and fermentation disappearance suggests an interaction between the two lines of reaction which leads to the establishment of a dynamic equilibrium of some kind. The second set of data shows conditions where the aerobic inhibition of increased fermentation is independent of the rate of respiration, which remains practically constant. This suggests a direct inhibition through oxygen. 
Table 4 surveys lactic and propionic acid bacteria and chlorophyllbearing plant tissue. The three types of lactic acid bacteria are representative examples which duplicate in every respect the metabolic types of the yeasts shown in Table 2. Lactobacillus delbrückii deserves special comment. It shows a well-developed

Table 4.-Bacterial and plant metabolism in glucose

\begin{tabular}{|c|c|c|c|c|c|c|}
\hline Material & Organism & $\mathrm{QO}_{2}$ & $\mathbf{Q}^{\mathrm{O}_{2}} \mathrm{~F}$ & $Q^{N_{2}} F$ & O.Q. & $\begin{array}{l}\text { Aerobic } \\
\text { inhibi- } \\
\text { tion } \\
\text { per cent }\end{array}$ \\
\hline \multirow{4}{*}{$\begin{array}{l}\text { Lactic acid } \\
\quad \text { bacteria (13) }\end{array}$} & Bacterium cereale & -189 & 49 & 305 & 3.9 & 84 \\
\hline & $\begin{array}{l}\text { Bacterium Delbrückii } \\
\text { (hemin-free) }\end{array}$ & -109 & 79 & 188 & 3 & 58 \\
\hline & Lactobacillus casei & 0 & 287 & 316 & & 9 \\
\hline & & -3 & 255 & 277 & 22 & 8 \\
\hline \multirow{2}{*}{$\begin{array}{r}\text { Propionic acid } \\
\text { bacteria (14) }\end{array}$} & Propionibacterium & & & & & \\
\hline & pentosaceum & -15 & 4 & 20 & 3 & 80 \\
\hline \multirow{2}{*}{$\begin{array}{l}\text { Lathyrus plant } \\
\text { (15) }\end{array}$} & Sprout & -3.5 & 0 & 1 & & \\
\hline & Leaf & -1.4 & 0 & 0.8 & & \\
\hline \multirow[t]{2}{*}{ Algae (15) } & Chlorella pyrenoidea & -5 & 0 & 1.6 & & \\
\hline & Coclastrum proboscideum & -12.7 & 0 & 2.7 & & \\
\hline
\end{tabular}

Pasteur effect without possessing any catalysts of the hemin type. The oxygen-producing plant tissues show only a small fermentative capacity.

\section{ANIMAL TISSUES}

The fairly frequent occurrence among invertebrates of organisms adapted to anaerobic life has been mentioned. Particularly among the worms is found a great variety of partly or wholly anaerobic forms. Here, on a higher phylogenetic level, appears a metabolic stratification similar to that in the yeasts and bacteria. There seems to be a gradual transition from the facultative anaerobic free-living worms to the obligate anaerobic parasitic forms (16). As early as 1909 Lesser (16a) made important quantitative experiments on the interrelation between respiration and fermentation in earthworm metabolism. He found that fermentation resulted in the consumption of from four to six times as much glycogen. These same ratios were later found by Meyerhof to hold likewise for frog

* This organism has been referred to by a variety of names in the biochemical literature, including: Bacterium Delbrïckii, Lactobacillus Delbrïkii, Bacterium cereale, Bacillus acidificans longissimus, etc. 
muscle (17). As end products in worm fermentation Lesser found, in addition to lactic acid, large amounts of higher fatty acids, especially valeric acid (cf. also 18 and 19). An increase of glycogen utilization in anaerobiosis is described for many other types of worms (19). The parasitic worms living in the practically oxygenfree intestinal fluids show predominantly anaerobic metabolism. Although they are able to respire aerobically, their fermentation does not seem to be inhibited nor their glycogen consumption diminished in oxygen (19).

The higher vertebrates and especially the warm-blooded animals must be considered as essentially aerobic organisms. This is not true for all their parts, however, nor under all conditions. The experiment shown in Figure 2 is an example of partial anaerobiosis: the muscle suddenly put under high strain must rely predominantly on a supply of anaerobic energy. In Table 5 metabolic figures for representative

Table 5.-Metabolism of animal tissues

\begin{tabular}{llcccccc}
\hline \hline Type & \multicolumn{1}{c}{ Tissue } & $\mathrm{RQ}$ & $\mathrm{Q}_{\mathrm{O}_{2}}$ & $\mathrm{Q}^{\mathrm{O}_{2} \mathrm{G}}$ & $\mathrm{Q}^{\mathrm{N}_{2} \mathrm{G}}$ & O.Q. & $\begin{array}{c}\text { Refer- } \\
\text { ence }\end{array}$ \\
\hline Largely a erobic & Kidney & 0.8 & -20 & 0 & $0-8$ & & 80 \\
& Liver & $0.6-0.9$ & -12 & $0-2$ & $0-12$ & & 80 \\
Facultative & Brain & 1 & -16 & 0 & 26 & 4.9 & 36 \\
anaerobic & Pituitary & - & -12 & 0 & 13 & 3.3 & 81 \\
& Testis & $0.7-1$ & -14 & 8 & 14 & 1.3 & 80 \\
& Medulla of kidneys & 0.97 & -9 & 16 & 23 & 3.3 & 20 \\
Largely anaerobic & Mucosa jejuni & 0.86 & -16 & 25 & 23 & 0 & 22 \\
& Sperm, human & - & -1 & 6.5 & 8 & & 82 \\
\hline
\end{tabular}

tissues are assembled. During recent years interesting examples of adult tissues with predominantly anaerobic metabolism have been described. Relatively large and aerobically persistent glycolysis has been found, for example, in the medulla of the kidney and in cartilage $(20,21)$. Dickens correlates the metabolic pattern with the relatively poor blood supply of these tissues. Large and aerobically persistent glycolysis in the intestinal mucosa recently reported by Dickens and Weil-Malherbe (22) might likewise be correlated with the previously mentioned lack of oxygen in the intestinal fluid surrounding it.

These observations show that the pronounced anaerobic metabolism of embryonic tissue and of malignant growth (Table 6) is not an isolated phenomenon. Here are tissues, as has been pointed 
out in an earlier paper (23), which through environmental conditions, such as insufficient circulation, are forced to rely partly on a supply of anaerobic energy (cf. 20). Recent measurements by Philip (24) on respiration of the early developmental stages of the chick embryo give evidence that this must be the case. This corroborates our earlier findings based on less conclusive experimental data. Philip's remarks may be quoted here: "The study of the early blastomere," he says,

Table 6.-Metabolism of tumor tissue

\begin{tabular}{|c|c|c|c|c|c|c|}
\hline Tumor & $\mathrm{QO}_{2}$ & $\mathbf{Q}^{\mathbf{O}_{2}} \mathbf{G}$ & $Q^{N_{2}} G$ & O.Q. & $\begin{array}{l}\text { Inhibi- } \\
\text { tion } \\
\text { per cent }\end{array}$ & $\begin{array}{l}\text { Refer- } \\
\text { ence }\end{array}$ \\
\hline $\begin{array}{l}\text { Flexner-Jobling carcinoma, } \\
\text { rat } . .\end{array}$ & $\begin{array}{l}-15 \\
-7\end{array}$ & $\begin{array}{l}23 \\
16\end{array}$ & $\begin{array}{l}23 \\
24\end{array}$ & $\begin{array}{l}0 \\
3\end{array}$ & $\begin{array}{r}0 \\
33\end{array}$ & 54 \\
\hline $\begin{array}{l}\text { Adenocarcinoma, human } \\
\text { male . . . . . . . }\end{array}$ & $\begin{array}{l}-9 \\
-1.2 \\
0\end{array}$ & $\begin{array}{r}16 \\
5 \\
9\end{array}$ & $\begin{array}{l}29 \\
12 \\
22\end{array}$ & $\begin{array}{l}4.2 \\
15 \\
\infty\end{array}$ & $\begin{array}{l}42 \\
58 \\
59\end{array}$ & 75 \\
\hline $\begin{array}{l}\text { Jensen sarcoma . . } . \cdots \\
\text { Walker sarcoma } 256\end{array}$ & $\begin{array}{l}-14 \\
-22\end{array}$ & $\begin{array}{l}16 \\
25\end{array}$ & $\begin{array}{l}34 \\
46\end{array}$ & $\begin{array}{l}3.9 \\
2.9\end{array}$ & $\begin{array}{l}53 \\
46\end{array}$ & $\begin{array}{l}39 \\
39\end{array}$ \\
\hline
\end{tabular}

The average R.Q. for all the tissues was 0.85 .

"has revealed that oxygen diffusion limits the oxygen consumption in oxygen tensions of the air. This indicates that the early blastomere may actually be in a state of partial anaerobiosis." And later in the same discussion: "The considerations presented suggest that some of the energy used during early periods of growth can be provided to the embryo by anaerobic processes. This condition is probably associated with the rapidly increasing size of the embryo during early periods before the circulation system can function as an adequate oxygenating mechanism." The relatively poor vascularization of most tumors is evidence that the last statement holds likewise for malignant growth. We are led then to the conclusion that the high capacity of the anaerobic metabolism present in normal and in malignant growing tissues should be attributed to their partly anaerobic state of life rather than to an unlikely special growth function of glycolysis.

A curious phenomenon of hyperfunction of the Pasteur effect is observed in human beings at high altitudes (25). The relatively high lactic acid level of the blood which would be expected at low oxygen pressure is observed only before adaptation occurs (25). 
After adaptation to the new environment the lactate level of the blood becomes normal. Even with exhausting work the lactic acid concentration remains very low, 2 to 3 millimolar, as compared with a blood level of 13 millimolar reached with exhausting work at sea level (Figure 2). Apparently a special mechanism prevents the muscle from utilizing too much of the anaerobic energy supply even at low oxygen pressure. Dill in his book on life at high altitudes (26) makes interesting comments on this phenomenon: "It is as though the body, realizing the delicacy of its situation with regard to oxygen supply, sets up an automatic control over anaerobic work which renders impossible the severe acid-base disturbances which can be voluntarily induced at sea level."

\section{INTERPREtation of the Pasteur EfFect}

During recent years discussion has centered more or less around the question whether the effect depends upon respiratory activity as such, or upon an inhibition produced by the action of oxygen. In the first case the rate of respiration with its output of energy would be a determining factor and a state of dynamic equilibrium would result; part or even all of the respiration energy would be spent or fixed to revert or repress glycolytic breakdown. If, however, the effect is brought about through oxygen, or more specifically by oxidative inhibition of an essential part of the glycolytic enzyme system, then the reaction may be independent of the rate of respiration and involve no transfer of energy.

\section{EQUILIBRIUM SCHEMES}

A fuller understanding of the partial reactions involved in fermentation and respiration has given new impulse to the discussion of their interrelationship as manifested in the Pasteur effect. The fact that cozymase and adenylic acid, the two transmitter substances in fermentation, are likewise participants in respiration has given rise to some interesting suggestions.

Ball (27) has pointed out that, aerobically, respiratory oxidants such as flavin may compete with pyruvic acid for the reduced cozymase. Pyruvic acid would disappear largely through oxidation rather than through fermentative reduction. Adler and Calvet (28), however, comparing the ratio of oxidized to reduced cozymase in aerobic and in anaerobic baker's yeast found no significant difference, but a ratio of nearly one to one in both cases.

Adenylic acid, the other common transfer system, was first linked. 
with the Pasteur effect through work done by Ostern and Mann (29). They found that the addition of adenosine triphosphate (ATP) to mashed muscle depressed aerobic glycolysis and raised the Meyerhof Quotient from 2.2 to 4. Later Lennerstrand (30) discussed the possibility that with aerobic over-phosphorylation of adenylic acid (Ad), the ratio ATP:Ad might become too high to permit adenylic acid to function effectively as a transmitter in fermentation.

A scheme based on the recent development of the biochemistry of phosphate turnover has been presented and discussed in detail elsewhere (3). Figure 3 is taken from this paper. The upper cycle, revolving clockwise, represents anaerobic glycolysis; the lower cycle, revolving counter-clockwise, represents aerobic resynthesis. It appears that the clockwise run of the glycolytic cycle depends on the outflow of the energy-rich phosphate created in the reaction. An actual reversal of the cycle back to the aldehyde stage may occur when through the aerobic influx of new energy-rich phosphate the carboxyl-bound phosphate in 1,3-diphosphoglyceric acid ( $\mathrm{Ph}$ glyceryl-Ph) cannot be removed.

In the Meyerhof-Warburg equilibrium reaction, as the diagram shows, inorganic phosphate is bound when the reaction proceeds to the right and is set free when it proceeds to the left. Therefore inorganic phosphate concentration can become a rate-determining factor. Meyerhof et al. (31) and Belitzer (32) have pointed out that in muscle the increased concentration of inorganic phosphate through creatinephosphate breakdown should be regarded as the cause of the release of metabolic activity due to stimulation. Along similar lines, Johnson (33) recently suggested that the lowering of inorganic phosphate concentration might be a possible cause of aerobic inhibition of glycolysis. Inorganic phosphate concentration seems, however, to be high in most cells except in resting muscle, where most of the phosphate is bound to creatine. Phosphate is generally considered to be the intracellular anion. How much of this is really free phosphate and how much is labile phosphate broken down by chemical manipulations remains to be determined (34).

In our opinion, the value of such schemes is limited because they disregard controlling factors in the cell which undoubtedly must regulate the routes of phosphate turnover-that is, the synthesis as well as the breakdown of intermediates. The Pasteur effect cannot be due merely to an "open" equilibrium; it must be due to specific transmitter systems. Evidence for this is the fact that the "linkage" 

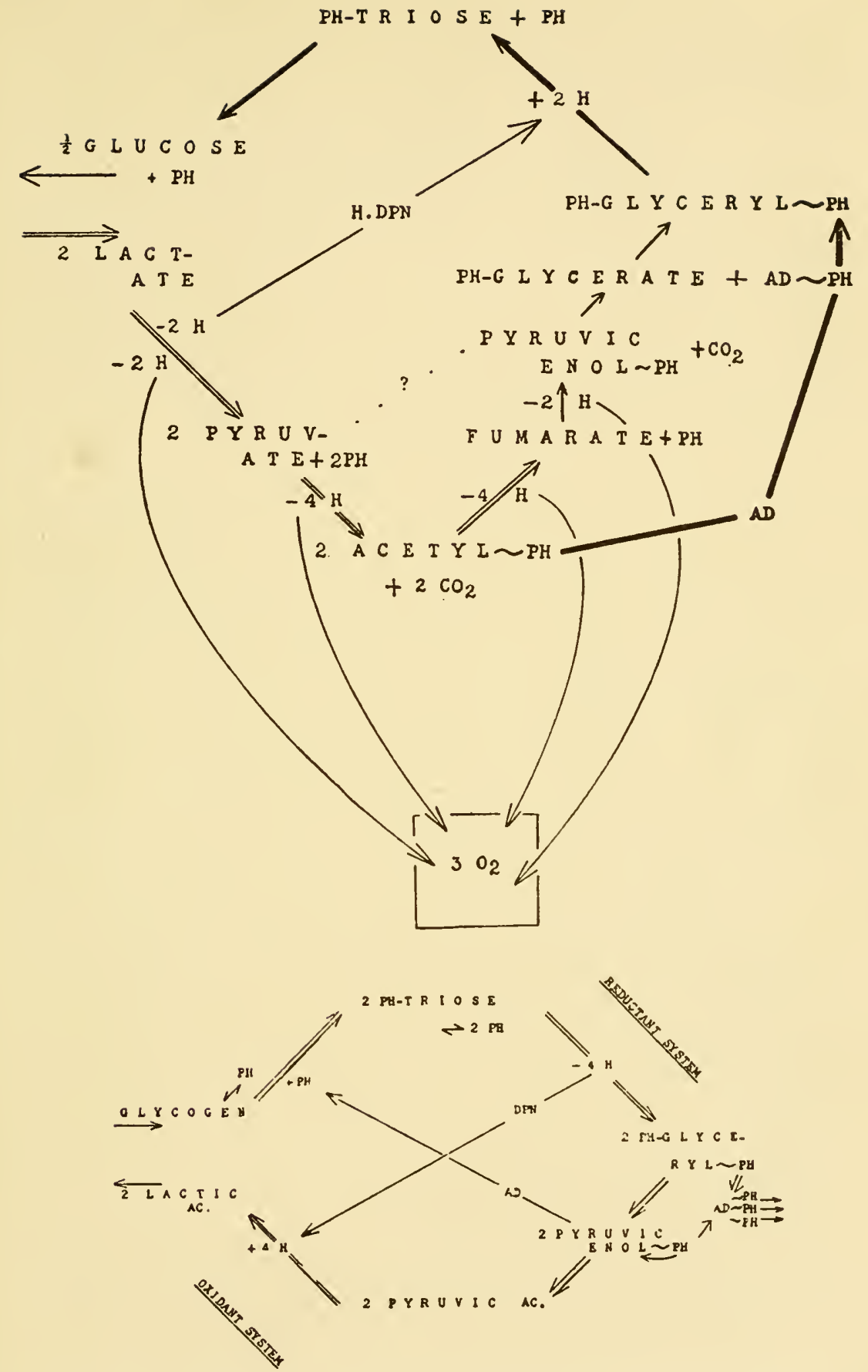

Figure 3.-Schemes for anaerobic breakdown (upper cycle) and aerobic resynthesis (lower cycle) of carbohydrate. (From F. Lipmann, Advances in Enzymology, vol. 1, 1941.) 
between respiration and glycolysis may be interrupted without impairing the reactions proper.

\section{INHIBITION OF THE PASTEUR EFFECT}

A considerable variety of compounds are able to interrupt the Pasteur effect, or the Pasteur reaction, as Warburg (35), after discovering the specific action of ethyl carbylamine, first called the phenomenon. Table 7 presents a survey of the agents which have been given the most study and which have proved most effective. Similar effects were found with phenylhydrazine by Dickens (36), with dinitrocresol and dinitrophenol by Dodds and Greville (41), and with HCN on certain plant cells by Genevois (42).

Table 7.-Aerobic release of glycolysis

\begin{tabular}{|c|c|c|c|c|c|c|}
\hline Inhibitor & $\begin{array}{c}\text { Concentra- } \\
\text { tion }\end{array}$ & $\mathrm{Q}_{2}$ & $\mathrm{Q}^{\circ} \mathrm{G}$ & R.Q. & Tissue & $\begin{array}{l}\text { Refer- } \\
\text { ence }\end{array}$ \\
\hline \multirow[t]{2}{*}{ Ethyl carbylamine } & 0 & -13 & 19 & & \multirow[t]{2}{*}{ Jensen sarcoma } & \multirow[t]{2}{*}{35} \\
\hline & $10^{-3} \mathrm{M}$ & -14 & 32 & & & \\
\hline Carbon monoxide, light & $88 \%$ & -11 & 6 & 0.72 & Allantois & 37 \\
\hline Carbon monoxide, dark & $88 \%$ & -11 & 11 & & & \\
\hline \multirow[t]{2}{*}{ Oxygen pressure } & $95 \%$ & -25.5 & 0 & 1.05 & \multirow[t]{2}{*}{ Chorion } & \multirow[t]{2}{*}{38} \\
\hline & $5 \%$ & -22 & 11 & 0.7 & & \\
\hline \multirow[t]{2}{*}{ Phenosafranine } & 0 & -13 & 1 & 1.03 & \multirow[t]{2}{*}{ Brain } & \multirow[t]{2}{*}{39} \\
\hline & $10^{-5} \mathrm{M}$ & -13 & 21 & 0.98 & & \\
\hline \multirow[t]{2}{*}{ Glutathione } & 0 & -230 & 74 & & \multirow[t]{2}{*}{ Yeast } & \multirow[t]{2}{*}{40} \\
\hline & $2.5 \times 10^{-3} \mathrm{M}$ & -205 & 255 & & & \\
\hline
\end{tabular}

The common effect is the release of aerobic glycolytic action up to an anaerobic level, while respiration remains quantitatively unchanged. In Laser's experiments $(37,38)$ with carbon monoxide and low oxygen pressure the respiratory quotient was lowered, indicating qualitative changes of respiration. With phenosafranine, however, Dickens (39) found that the respiratory quotient of the brain remained unity and the manometric and chemical determinations of lactic acid were in excellent agreement. In this case, at least, it seems very probable that the interruption of the Pasteur reaction occurred without a qualitative change of respiration.

The action of metal-specific inhibitors has been of great interest. Work in this field has revived discussion of the question whether the effect is dependent on, or independent of, respiratory activity. The old observation that in most tissues cyanide released aerobic 
fermentation by inhibiting respiration was taken as indisputable proof of the dependence of the Pasteur effect on the intactness of respiration. Consequently ethyl carbylamine action, affecting only the Pasteur effect, but leaving primary respiration intact, was interpreted as inhibition of a reaction linking respiration to glycolysis.

A differential inhibition of respiration and Pasteur reaction by carbon monoxide was observed by Warburg (43a) in yeast experiments. Mainly interested in the respiratory effect of carbon monoxide, he remarked only incidentally upon the relatively higher sensitivity of the Pasteur reaction. Later, Laser (38) showed that in animal tissues the differences in sensitivity were pronounced. Frequently, he found, carbon monoxide had little or no effect on respiration but did cause aerobic glycolysis to appear. The release of aerobic glycolysis in animal tissues had been observed by Warburg and Negelein (43), but had been considered as a secondary effect due to inhibited respiration. From some preliminary measurements of the effect of light on aerobic glycolysis in retina in the presence of carbon monoxide, the spectrum of the Atmungsferment was charted. Since Laser (38) had found respiration in retina to be uninfluenced by carbon monoxide, these measurements, as Stern and Melnick (44) recognized, had to be reinterpreted as preliminary measurements of the spectrum of the Pasteur agent-carbon monoxide compound. Stern and Melnick then measured carefully with the Warburg illumination technique the relative absorption spectrum of the Pasteur agent-carbon monoxide compound. The decrease in aerobic fermentation on irradiation was plotted against wave length. This decrease may be assumed to be due to the decomposition of the Pasteur agent-carbon monoxide complex. The resulting spectrum was very similar to that of the respiratory enzyme. Such measurements were made on retina (44) and yeast (45). Recently Melnick (45a) charted the spectrum of the respiratory enzyme of animal tissue by using heart muscle extracts in which, in contrast to the intact tissue, respiration is sensitive to carbon monoxide (46). The bands developed from these measurements are reproduced in Figure 4. The spectra of the respiratory enzymes in yeast and in animal tissue, respectively, differ greatly, as do those of the Pasteur enzymes. In each case, however, the spectrum of the Pasteur enzyme follows closely that of the respiratory enzyme, deviating only in the absorption at longer wave lengths. The consistent, although small, differences are considered as evidence of the existence of two 
definitely different enzymes, one catalyzing the final step in the oxidation of metabolites, the other catalyzing the oxidative inactivation of a part of the glycolytic system.

This analysis seems a very promising approach at least to an elucidation of the events taking place on the oxygen side. The similarity between the respiratory and Pasteur enzymes suggests a direct
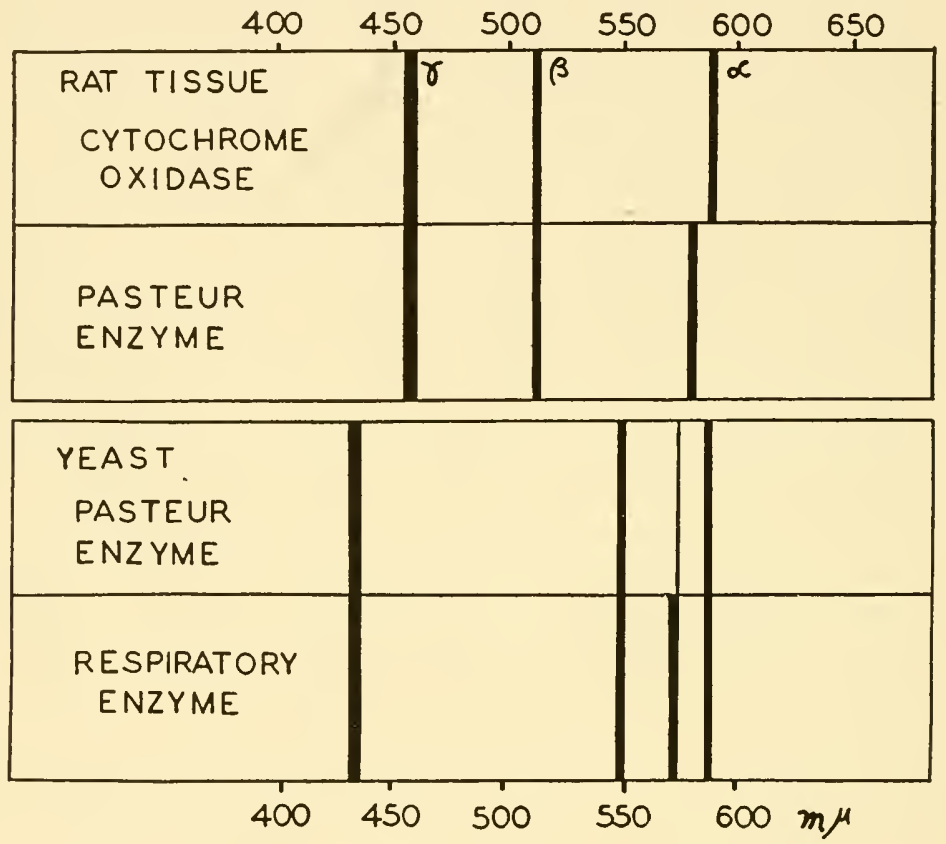

Figure 4.-Spectra of the respiratory and Pasteur enzymes in animal tissue and in yeast

reaction between oxygen and the transmitter. This is further indicated by the difference in the affinity of the two enzymes for oxygen $(37,47,76)$. The peculiarities of the carbon monoxide effect on respiration and a change of respiratory quotient at low oxygen tension (37) and with carbon monoxide (38), however, seem to indicate that the present interpretation may not represent the final solution.

In spite of the interest that attaches to the metal-specific anticatalysts, it should not be overlooked that such inhibitors as phenosafranine, dinitrocresol (41), and glutathione can hardly be considered metal-specific. In an extensive study of the action of pheno- 
safranine and other phenazine derivatives, Dickens $(39,48)$ presents evidence which suggests that a flavin enzyme may participate in the transmission of the aerobic inhibition. The relationship between flavin and the Pasteur effect is indicated also by its occurrence in the hemin-free Lactobacillus delbriickii (13), where flavin is the only respiratory catalyst.

The disturbance of the Pasteur effect in brain that attends a lack of ionic balance represents a phenomenon of great complexity. Ashford and Dixon (49) observed a profound metabolic change in brain slices suspended in tenth molar potassium chloride. Aerobically they found increased respiration and appearance of glycolysis; and anaerobically, gradual and irreversible disappearance of glycolysis. They correlated the metabolic changes with the well-known increase in cell permeability through potassium ion (50). Dickens and Greville (51) showed subsequently that the potassium effect is specific for brain and is not found in other tissues, and that omission of calcium had a similar effect. Continuing on similar lines, WeilMalherbe (52) observed definite effects of potassium and also ammonium ions at much lower concentrations than those used by Ashford and Dixon.

This effect of electrolyte on brain metabolism signifies a great lability of the Pasteur mechanism. Warburg (54) has emphasized that the Pasteur mechanism is universally very sensitive to unphysiological surroundings. For example, in rat embryo aerobic glycolysis is high in Ringer solution but low or absent in serum or amniotic fluid. Effects of this type must be taken as an indication that aerobic disappearance of glycolysis is the result of an easily disturbed balance of reactions.

\section{REVERSIBLE OXIDATIVE INHIBITION OF GLYCOLYSIS IN EXTRACTS}

In order to approach experimentally the possibility that oxidative inhibition might be the cause of aerobic disappearance of fermentation, I studied some time ago the effect of oxidizing agents on glycolysis and fermentation in extracts $(55,56)$. It was shown that the fermenting system was inactivated by small amounts of iodine and quinone. By adding indophenols as oxidants inhibition in oxygen was provoked, which disappeared in its absence, when the oxidizing dye was reduced by constituents of the extract and through its enzymatic activity. Two experiments of this type with muscle extract and yeast juice, respectively, are summarized in Table 8. It appears that addition of the dye reproduces a Pasteur effect, which occurs, 
however, with negligible respiratory activity, demonstrating the possibility of a reversible oxidative inactivation.

The inhibition of glycolysis with iodine was subsequently studied more carefully by Gemmill and Hellerman (57). With concentrations just high enough to obtain fairly complete inhibition they were able to recover the activity by adding glutathione or cysteine. This suggested strongly that the oxidative inhibition was due to reversible oxidation of enzyme SH-groups. Rapkine $(58,59)$ then showed that the oxidoreduction between phosphoglyceraldehyde and pyruvic acid was at least one of the partial reactions being blocked by oxidation, presumably of enzyme-SH. This reaction system could be inactivated by $\mathrm{S}$-S-glutathione and reactivated by SH-glutathione. More recently Rapkine found the same reaction reversibly inacti-

Table 8.-Induced Pasteur effect in cell extracts

\begin{tabular}{|c|c|c|c|c|c|c|c|}
\hline \multirow[t]{2}{*}{ Extract } & \multirow[t]{2}{*}{ Addition } & $\mathrm{O}_{2}$ & $\begin{array}{c}\mathrm{CO}_{2} \text { in } \\
\mathrm{O}_{2}\end{array}$ & $\begin{array}{c}\mathrm{CO}_{2} \text { in } \\
\mathrm{N}_{2}\end{array}$ & \multirow[t]{2}{*}{ O.Q. } & \multirow{2}{*}{$\begin{array}{l}\text { Aerobic } \\
\text { inhibi- } \\
\text { tion } \\
\text { per cent }\end{array}$} & \multirow[t]{2}{*}{ Ref. } \\
\hline & & \multicolumn{3}{|c|}{ Cubic millimeters per hour } & & & \\
\hline \multirow[t]{2}{*}{ Yeast } & $\begin{array}{l}6 \times 10^{-3} \text { M naphthol- } \\
\text { sulfonate indo- } \\
\text { phenol }\end{array}$ & -49 & 106 & 710 & 37 & 85 & 56 \\
\hline & None & - & 1140 & 1120 & - & 0 & \\
\hline \multirow[t]{2}{*}{ Muscle } & $\begin{array}{l}10^{-3} \mathrm{M} \text { dichloro- } \\
\text { phenol indophenol }\end{array}$ & -4 & 20 & 380 & 270 & 95 & 55 \\
\hline & None & - & 440 & 425 & - & 0 & \\
\hline
\end{tabular}

vated by dichlorophenolindophenol (personal communication), which might explain my earlier results with the complete glycolytic system. With these experiments the possibility of a reversible oxidative inactivation has become firmly established. It is therefore of little significance for the question at issue that, as was shown by Michaelis and Smythe (60), many dyes, irrespective of oxidation-reduction potential, inhibit irreversibly by various mechanisms, or that naphtholsulfonate indophenol with different yeast preparations leads earlier to irreversible inactivation than in our experience.

Besides the system studied by Rapkine, a number of partial enzymes of glycolysis were found to undergo oxidative inactivation followed by reactivation with glutathione. These reactions are as follows: 
phosphoglyceraldehyde + pyruvate $\rightarrow$ phosphoglycerate + lactate (58)

glycogen + phosphate $\leftrightarrows$ glucose-1-phosphate $(61,62)$

glucose-1-phosphate $\rightleftarrows$ glucose-6-phosphate (62)

adenosinediphosphate + glucose $\rightarrow$ adenylic acid + glucose-6phosphate (63)

The activation by thiol compounds of glycolysis in extracts, described by Geiger and Magnes (64) and Michaelis and Runnström (65) thus becomes easily understandable.

\section{THIOL INFLUENCE ON FERMENTATION AND GLYCOLYSIS}

\section{IN INTACT CELLS}

The function of glutathione is not yet well understood. It is present in practically every cell in fairly large amounts. Frequently it has been suggested that it performs the role of an oxidationreduction buffer. The very complexity of intracellular metabolism prevents us from making more than vague statements of that type. The protection against oxygen injury which thiol compounds give to strict anaerobes, first observed by Quastel and Stephenson (53), lends support to the assumption that their function is one of stabilization.

Observations on intact cells as well as on cell-free enzyme systems suggest a regulatory effect of thiol compounds on glycolysis and fermentation. As yet it is impossible to correlate definitely the action on intact fermenting cells and on fermenting enzyme extracts or partial systems, but a promising approach seems to be opened which is worth very careful consideration.

Release of aerobic glycolysis with glutathione was first observed by Bumm and Appel (66) with sliced animal tissues. Soon afterward Quastel and Wheatley (40) made an interesting study of the effects of glutathione and cysteine on the metabolism of baker's yeast. One of their experiments is included in Table 7 above. Glutathione interrupts the Pasteur effect without affecting respiration. They noted that an extract of brewer's yeast had much the same effect as glutathione, which they ascribe to the large content of thiol compounds in brewer's yeast. With cysteine the effect on aerobic glycolysis was the same, but respiration was markedly inhibited. The respiratory inhibition was specific for glucose and absent when glycerol was used as substrate. More recently Runnström and Sperber (67) undertook a study of the cysteine effect. Accompanying 
the release of aerobic fermentation they found an inhibition of the synthesis of higher carbohydrates from glucose. This interesting observation would be still more significant if respiration were not inhibited at the same time. The alternative between glucose fermentation and synthesis to glycogen suggests that thiol compounds are able to upset the normal aerobic reaction course from glucose-6phosphate over Cori-ester to glycogen, forcing the glucose monoester into the fermentation cycle.

With Propionibacterium pentosaceum a number of interesting observations were made by Fromageot and Chaix (14). Dilute suspensions of repeatedly washed bacteria did not ferment in the presence of minute amounts of oxygen. This inhibition was counteracted by very small concentrations of cysteine or hydrogen sulfide. With unwashed and concentrated suspensions small amounts of oxygen did not affect the fermentation, but aerobically fermentation disappeared (normal Pasteur effect, Table 4) and was released by thiol compounds. They concluded that normally a substance is present in bacteria which protects the fermentation system against the action of small amounts of oxygen. Since with impoverished organisms protection can be restored with cysteine, they assumed the protecting substance to be a thiol compound. With high oxygen pressure the physiological concentration of the protective system is not high enough to counteract the oxidative inhibition and aerobic disappearance of fermentation; that is, the Pasteur effect occurs. When the concentration of thiol compound is increased, the oxidative inhibition is blocked again, and aerobic fermentation appears. In other words, the occurrence of fermentation depends on the relative concentrations of SH-compound and oxygen, respectively.

An observation reported by Dickens (68) with pyocyanine should be mentioned here. In the presence of this dye "anaerobic" glycolysis of sarcoma was inhibited when measured in unpurified nitrogen containing 0.3 per cent oxygen. At the same time a slight color remained, indicating slight reoxidation of the dye. The color and the inhibition disappeared when chromous chloride was used to absorb the traces of oxygen. The parallel between this phenomenon and our dye-induced Pasteur effect in extracts, as well as Fromageot's effect of low oxygen pressure on propionic acid bacteria, is obvious.

Despite the complexity of dye effects on living cells $(68,69)$, Dickens came to the conclusion that in general there is a tendency for dyes with high oxidation-reduction potential to increase the 
Pasteur effect. In harmony with this generalization is the increase of the Pasteur effect in tumors by ferricyanide (70) and in yeast by indophenols (71). The complexity of the dye effects, however, is illustrated by the action of methylene blue, which, according to early observations by Gerard (72), releases aerobic glycolysis in muscle, while it was found by Barron (73) to inhibit aerobic glycolysis in erythrocytes. Nevertheless there seems to be a parallelism between dye action in extracts and in cells and a correlation between thiol and dye effects.

\section{PASTEUR EFFECT IVITH VERY LOW RESPIRATION}

That aerobic inhibition of fermentative metabolism is independent of respiration can be most clearly demonstrated through the occurrence of the Pasteur effect with very low respiration. Aside from the dye-induced Pasteur effect in extracts, some examples of such phenomena in living cells have already been discussed, such as inhibition by traces of oxygen or inhibition in the presence of relatively low respiration in yeast $(11,12)$. As a rule parallelism between the appearance of anaerobic metabolism and the disappearance of respiration is to be expected by the very nature of the phenomenon. The fact that the most-used inhibitors of respiration are metal-specific and are likewise more or less pronounced inhibitors of the Pasteur reaction, has greatly complicated the analysis. Inhibitors of respiration which interrupt the chain, not at

Table 9.-Pronounced Pasteur effect with very low respiration

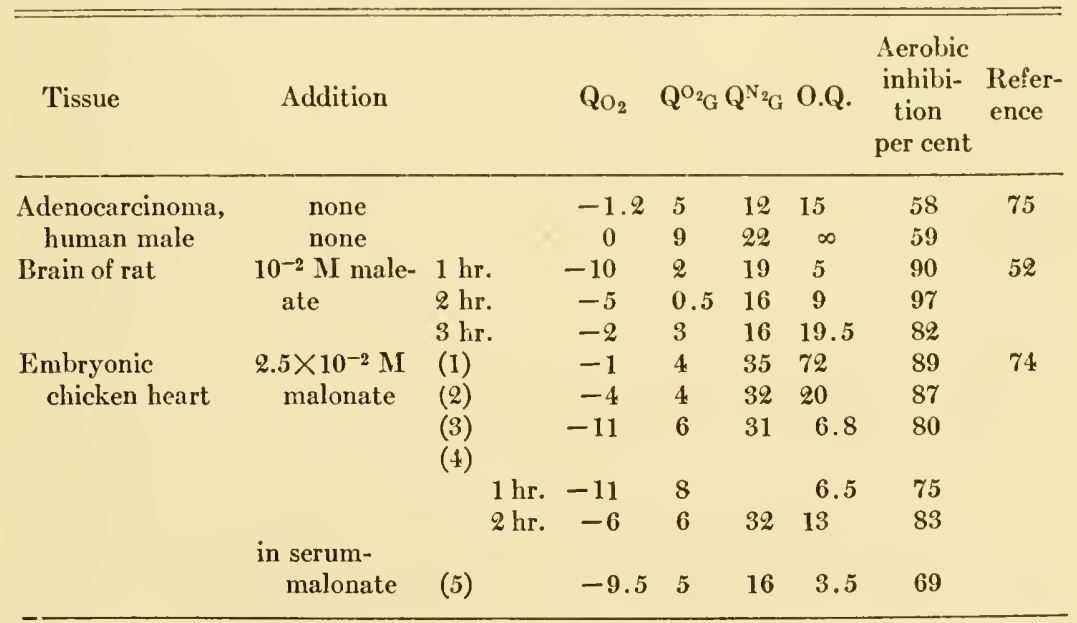


the end where the iron catalysts are operating but at an earlier stage, might be expected under favorable conditions to interrupt respiration without affecting the Pasteur reaction. Malonate and maleate, which block the Szent-György cycle, might react in this way. Weil-Malherbe (52) has indeed found with maleate poisoning that there is no appreciable aerobic glycolysis in brain when respiration has already declined to very low levels. I found, with malonate, similar effects on embryonic heart (74). In Table 9 a survey is given of these and other experiments, where with animal cells a Pasteur effect was found with low respiration. This was observed by Rosenthal and Lasnitzki (75) with some human cancer without inhibitors and by Kempner and Gaffron (76) with myeloblasts at 6 per cent oxygen pressure. It should be remembered that in Kempner's experiments with myeloblasts, while the Pasteur reaction was unaffected, respiration declined with falling oxygen pressure: the $\mathrm{Q}_{o_{2}}$ in 95 per cent oxygen was 8 ; in 6 per cent oxygen, 3.2. Laser (37) found the reverse with chorion, retina, and mouse liver; that is, little influence of low oxygen tension on respiration but inhibition of the Pasteur reaction.

Malonate does not have the effect described above on all tissues. I found with pigeon brain a decrease of respiration accompanied by a large increase of aerobic glycolysis (74). Similar results were reported by Kutscher and Sarreither (77) with skeletal muscle.

\section{Conclusion and OUTLOOK}

It has not been my purpose to give a complete survey of the work in this field. The recent reviews by Burk $(83,84)$ constitute a competent discussion of the problem as a whole, especially with regard to earlier work and thoughts. Our purpose here has been to summarize mainly the facts that indicate the occurrence of an oxidative inhibition. In general the evidence may be considered indicative but not conclusive, except in a few instances. The cell may choose to eliminate unneeded anaerobic metabolism by an inhibitory mechanism rather than by a counterforce, but there are indications that such inhibition acts upon reactions directing the internal flow of energy. Substances interrupting the Pasteur linkage likewise interrupt synthetic reactions, as has been shown in the case of cysteine (67) and especially dinitrophenol (41). Clifton (78), while in Kluyver's laboratory, made the discovery that dinitrophenol inhibits completely the synthetic processes in microorganisms. His study was based on the work of Barker (85), who demonstrated that with 
"resting" organisms only part of the disappearing non-nitrogenous metabolite could be accounted for by oxidation, while a large part was converted into cell material, presumably carbohydrate. This conversion was completely interrupted in the presence of dinitrophenol, in which case catabolic breakdown continued until all material was oxidized $(78,79)$.

Dinitrophenol has therefore become an important tool for the study of the relation between anabolic and catabolic processes, which must be determined by the flow of energy-carrying reactions. In a recent paper (3) where I have discussed the generation and transfer of energy-rich phosphate bonds it is stated that a major part of metabolically yielded energy is converted primarily into phosphate bond energy. An understanding of the means by which the cell directs the flow of energy-rich phosphate bonds into predetermined reactions should lead to a more precise understanding of the mechanism of regulative cell reactions such as the Pasteur effect and the probable related action of the hormones.

\section{REFERENCES}

1. BANG, O., Dissertation, Copenhagen, 1935.

2. Burk, D., Proc. Royal Soc. (London), B 104, 153 (1929).

3. Lipmann, F., Advances in Enzymology, 1, 99 (New York, 1941).

4. OсhоA, S., J. Biol. Chem., 138, 751 (1941).

5. Belitzer, V. A., and Tzibakova, E. T., Biokimia, 4, 516 (1939).

6. Meyerhof, O., Biochem. Z., 162, 43 (1925).

7. Warburg, O., and Kubowitz, F., Biochem. Z., 189, 242 (1927).

8. Lipmann, F., Skand. Arch., 76, 255 (1937).

9. Nakashima, M., Biochem. Z., 204, 479 (1928).

10. Warburg, O., Biochem. Z., 189, 350 (1927).

11. Meyerhof, O., and Iwasaki, K., Biochem. Z., 226, 16 (1930).

12. Burk, D., Winzler, R. T., and du Vigneaud, V., J. Biol. Chem., 140, Proc., xxi (1941).

13. Davis, J. G., Biochem. Z., 265, 90; 267, 357 (1933).

14. Fromageot, C., and Chaix, P., Enzymologia, 3, 288 (1937).

15. Genevois, L., Biochem. Z., 186, 461 (1927).

16. v. Bunge, G., J. Physiol. Chem., 12, 565 (1888).

16a. Lessen, E. J., Ergebnisse d. Physiol., 8, 742 (1909).

17. Meyerhof, O., Pflügers Archiv., 185, 11 (1920).

18. Slater, W. K., Biochem. J., 19, 604 (1926).

19. v. Brand, T., Ergebnisse Biol., 10, 37 (1934).

20. Dickens, F., and Weil-Malmerbe, H., Biochem. J., 30, 659 (1936).

21. Dickens, F., and Weil-Malherbe, H., Nature, 138, 125 (1936).

22. Dickens, F., and Weil-Malherbe, H., Biochem. J., 35, 7 (1941).

23. Lipmann, F., Biochem. Z., 261, 157 (1933).

24. Philips, F. S., J. Exp. Zoology, 86, 257 (1941).

25. Edwards, H. T., Am. J. Physiol., 116, 367 (1936).

26. Dirl, D. B., Life, Heat and Altitude, p. 173 (Harvard University Press, 1938). 
27. Ball, E. G., Bull. Johns Hopkins Hosp., 65, 253 (1939).

28. Adler, E., and Calvet, F., Arkiv Kemi, Mineral. Geol., 12B, no. 32 (1936).

29. Ostern, P., and Mann, T., Biochem. Z., 276, 408 (1935).

30. Lennerstrand, A., Naturwissenschaften, 25, 347 (1937).

31. Meyerhof, O., Schulz, W., and Schuster, P., Biochem. Z., 293, 309 (1937).

32. Belitzer, V. A., Enzymologia, 6, 1 (1939).

33. Johnson, M. J., Science, 94, 200 (1941).

34. Lipuiann, F., J. Biol. Chem., 134, 463 (1940).

35. Warburg, O., Biochem. Z., 172, 432 (1926).

36. Dickens, F., Biochem. J., 28, 537 (1934).

37. LAser, H., Biochem. J., 31, 1671 (1937).

38. Laser, H., Biochem. J., 31, 1677 (1937).

39. Dickens, F., Biochem. J., 30, 1233 (1936).

40. Quastel, J. H., and Wheatley, A. H. M., Biochem. J., 26, 2169 (1932).

41. Dodds, E. C., and Greville, G. D., Lancet, 112: I, 398 ( 1934).

42. Genevois, L., Biochem. Z., 191, 147 (1927).

43. Warburg, O., and Negelein, E., Biochem. Z., 214, 64 (1929).

43a. Warburg, O., Biochem. Z., 177, 471 (1925).

44. Stern, K. G., and Melnick, J. L., J. Biol. Chem., 139, 301 (1941).

45. Melnick, J. L., J. Biol. Chem., 140, Proc., xc (1941).

45a. Melnick, J. L., Science, 94, 118 (1941).

46. Keilin, D., and Hartree, E. F., Proc. Roy. Soc. (London), B 127, 167 (1939).

47. Bumm, E., Appel, H., and Fehrenbach, K., Z. physiol. Chem., 223, 207 (1934).

48. Dickens, F., and McIlwain, H., Biochem. J., 32, 1615 (1938).

49. Ashrond, C. A., and Dixon, K. C., Biochem. J., 29, 157 (1935).

50. Duxon, K. C., Nature, 137, 742 (1937); Biol. Rev., 12, 431 (1937).

51. Dickens, F., and Greville, G. D., Biochem. J., 29, 1468 ( 1935 ).

52. Weil-Malherbe, H., Biochem. J., 32, 2257 (1938).

53. Quastel, J. H., and Stephenson, M., Biochem. J., 20, 1125 (1926).

54. Warburg, O., Stoffwechsel der Tumoren (Berlin, 1926).

55. LiplianN, F., Biochem. Z., 265, 133 (1933).

56. Lipmiann, F., Biochem. Z., 268, 205 (1934).

57. Gemmill, C. L., and Hellerman, L., Am. J. Physiol., 120, 522 (1937).

58. Rapkine, L., Biochem. J., 32, 1729 (1938).

59. Rapkine, L., and Trpinac, P., Compt. rend. soc. biol., 130, 1516 (1939).

60. Michaelis, L., and Smythe, C. V., J. Biol. Chem., 113, 717 (1936).

61. Gill, D. M., and Lehniann, H., Biochem. J., 33, 1151 (1939).

62. Cori, G. T., and Coni, C. F., J. Biol. Chem., 135, 733 (1940).

63. Colowick, S. P., and Kalckar, H. M., J. Biol. Chem., 137, 789 (1941).

64. Geiger, A., and Magnes, J., Biochem. J., 33, 866 (1939).

65. Michaelis, L., and Runnström, J., Proc. Soc. Exp. Biol. Med., 32, 343 (1935).

66. Bumin, E., and Appel, H., Z. physiol. Chem., 210, 79 (1932).

67. Runnström, J., and Spenber, E., Nature, 141, 689 (1938).

68. Dickens, F., Biochem J., 30, 1064 (1936).

69. Elliott, K. A. C., and Baker, Z., Biochem. J., 29, 2396 (1935).

70. Mendel, B., and Strelitz, F., Nature, 140, 771 (1937).

71. Hoogerheide, J. C., Dissertation, Leiden, 1935.

72. Geraro, R. W., Am. J. Physiol., 97, 523 ( 1931).

73. Barnon, E. S. G., J. Biol. Chem., 81, 445 (1929).

74. Lipmann, F., unpublished. 
75. Rosenthal, O., and Lasnitzki, A., Biochem. J., 196, 340 (1928).

76. Kempner, W., and Gaffron, M., Am. J. Physiol., 126, 553 (1939).

77. Kurscher, W., and Sarreither, W., Z. physiol. Chem., 265, 152 (1940).

78. Clifton, C. E., Enzymologia, 4, 246 (1937).

79. Doudoroff, M., Enzymologia, 9, 59 (1940).

80. Elliott, K. A. C., Greig, M. E., and Benoy, M. P., Biochem. J., 31, 1003 (1937).

81. Fujita, A., Biochem. Z., 197, 75 (1928).

82. MacLeod, J., Am. J. Physiol., 132, 190 (1941).

83. Burk, D., Occasional Publications, Am. Assoc. Adv. Sci., 4, 121 (1937).

84. Burk, D., Cold Spring Harbor Symp., 7, 420 (1939).

85. Barker, H. A., J. Cell. Comp. Physiol., 8, 231 (1936). 


\title{
Oxidases, Peroxidases, and Catalase
}

\author{
KURT G. STERN \\ Yale University School of Medicine
}

$\mathrm{N}$ THIS PAPER no attempt will be made to treat the subject in a
comprehensive or systematic manner. A number of review articles and monographs $(4,49,50,51,62,84,85)$ have been written on hemin catalysts and respiratory enzymes, to which the reader is referred for information on historical developments, basic facts, and details. By way of introduction some of the available data on hemin catalyses and on hemin-containing enzymes are given in Tables 1 to 5 , which are documented by references to experimental studies.

The chief aims of this presentation are to bring out certain fundamental features shared by all the catalysts under discussion; to analyze critically some of the controversial issues in the field; and to trace some of the more recent developments. To conserve space and to avoid overlappings with other papers, enzymes such as polyphenoloxidases, which contain copper rather than iron in their prosthetic group, and the cytochromes, which cannot be regarded as independent enzymes, will be considered only in so far as their relationship to the hemin enzymes may require it.

\section{The Common Denominator in Hemin Catalyses}

All reactions and catalyses in which hemins participate are either of the oxidative type or at least involve oxygen as a reactant. Under this heading are grouped a variety of processes, ranging from the transport of molecular oxygen by the respiratory pigments to the activation of oxygen or the transfer of electrons from ferrous to ferric iron. The few reports which have claimed that hemins or porphyrins have promoted hydrolytic reactions remain unconfirmed. The primary step in many hemin catalyses appears to be an interaction between coordinatively linked, porphyrin-bound iron and the bond between two oxygen atoms as it exists either in molecular oxygen or in the form of a peroxide bridge. This elementary process precedes or, indeed, represents what is mysteriously called the phenomenon of "oxygen activation." All that we know about it is that oxygen atoms thus captured acquire a state of high reactivity. The 
explanation that this activation is caused by a "deformation of the electron shells of the oxygen atom" is little more than a clever means of hiding our ignorance about a process which in biological import may be likened to the primary reaction of carbon dioxide with chlorophyll during photosynthesis. It is much easier to comprehend the type of interaction represented by the oxidation of ferrocytochrome by the ferri form of the respiratory ferment of Warburg. This reaction must be determined, at least partly, by a difference in oxidation-reduction potential between the two iron compounds, although it is not absolutely necessary that the normal potential of the Warburg enzyme be considerably more positive than that of the cytochrome. We know of instances where the reduced form of a reversible system is oxidized partly by the oxidized form of a more negative system, such as the methemoglobin formation by methylene blue. The extent of such an interaction is governed by rigid thermodynamic principles only in isolated and homogeneous systems where no side reactions take place. In a living cell, where the ferrous form of the respiratory enzyme is rapidly reoxidized by molecular oxygen and the ferri form of cytochrome is rapidly reduced by Hogness and Haas's "cytochrome reductase," the ratio of the various forms, in the steady state of respiration, may differ considerably from that in the isolated system.

The catalytic power of hemins is, in the last analysis, a function of the catalytic power of the central iron atom. The schema, traced in Figure 1, illustrates how the highly complex and specialized hemin protein enzymes stem from iron in its simplest form. In the lowly iron sulfate we already encounter, in a rudimentary form, some of the features which distinguish the oxidases, peroxidases, and catalase. It is fascinating to watch the increase in catalytic activity and the increasing degree of specialization that takes place as the iron atom is riveted into compounds of increasingly complex structure. In many respects hemin occupies a central position in the scheme. In its linkage to the porphyrin skeleton the iron atom reaches a new level of catalytic activity. Under conditions where simple iron salts decompose $10^{-5}$ moles of hydrogen peroxide, hemin will split $10^{-2}$ moles, representing a thousandfold gain. That the essential feature here is the iron-nitrogen-carbon bridge is indicated by Warburg's classical experiments with charcoal prepared from hemin, where this bridge apparently remains standing among the ruins of the iron-porphyrin ring system and imparts a power to oxidize cysteine and amino acids which is vastly superior to that 


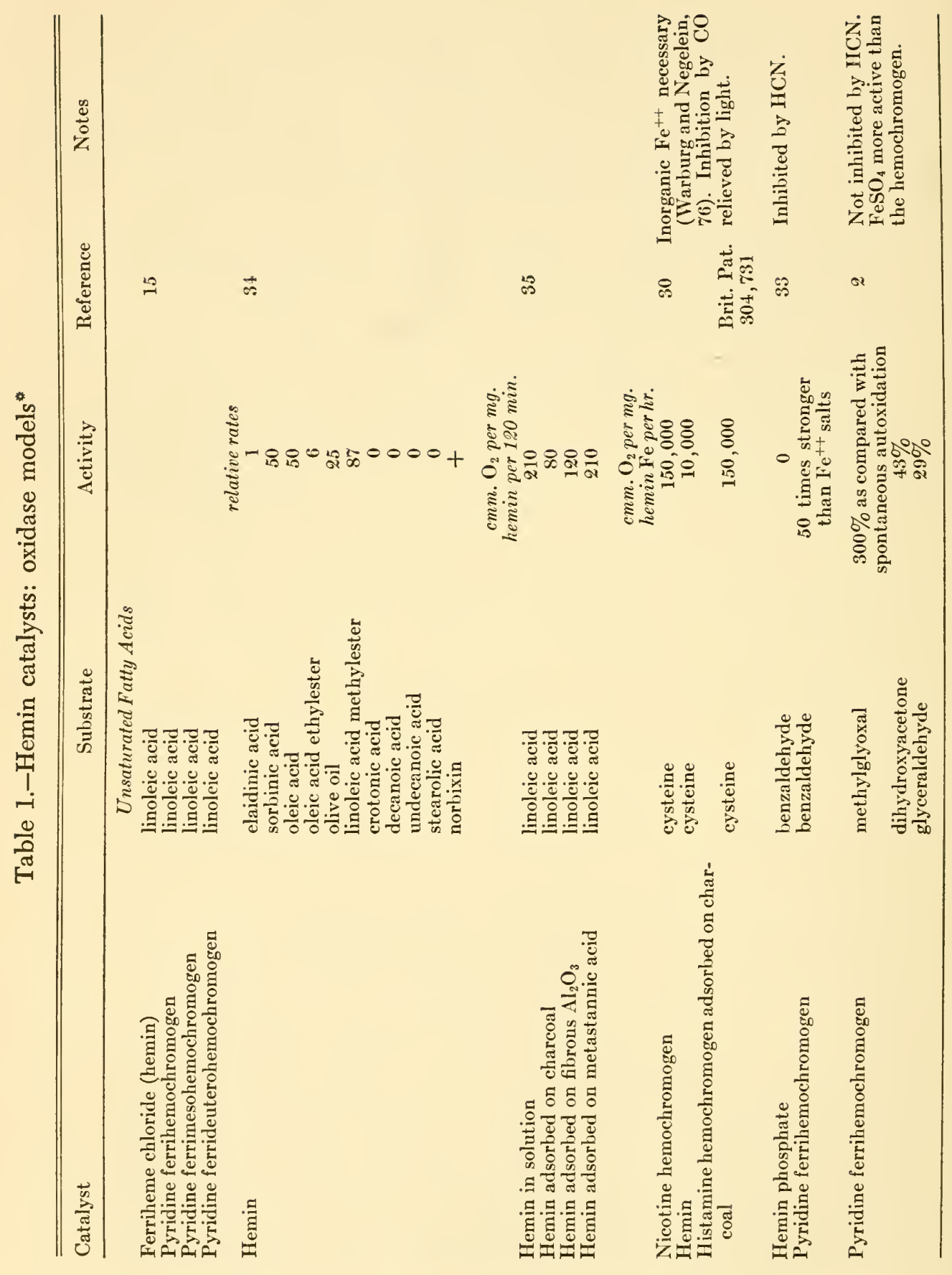




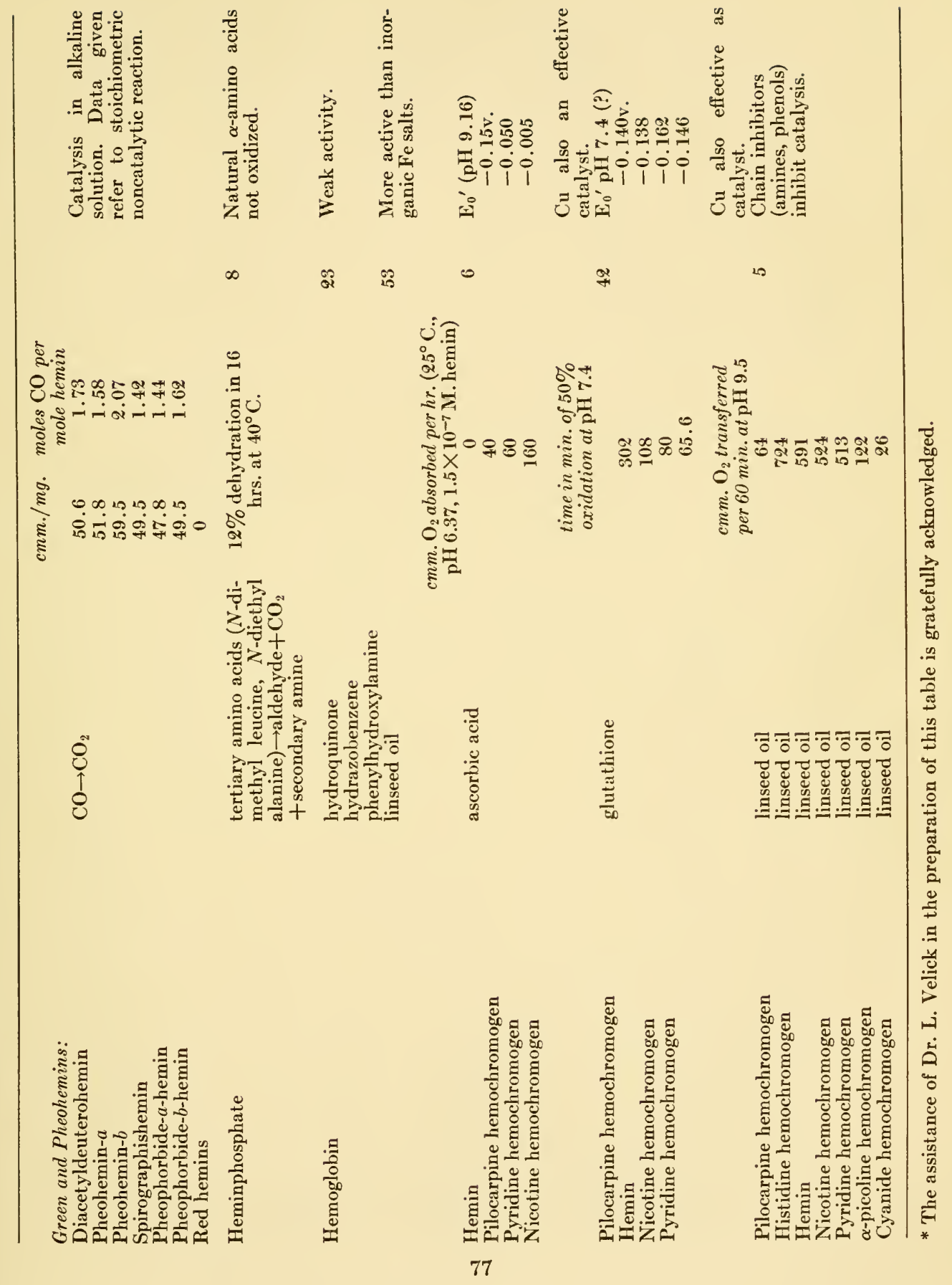




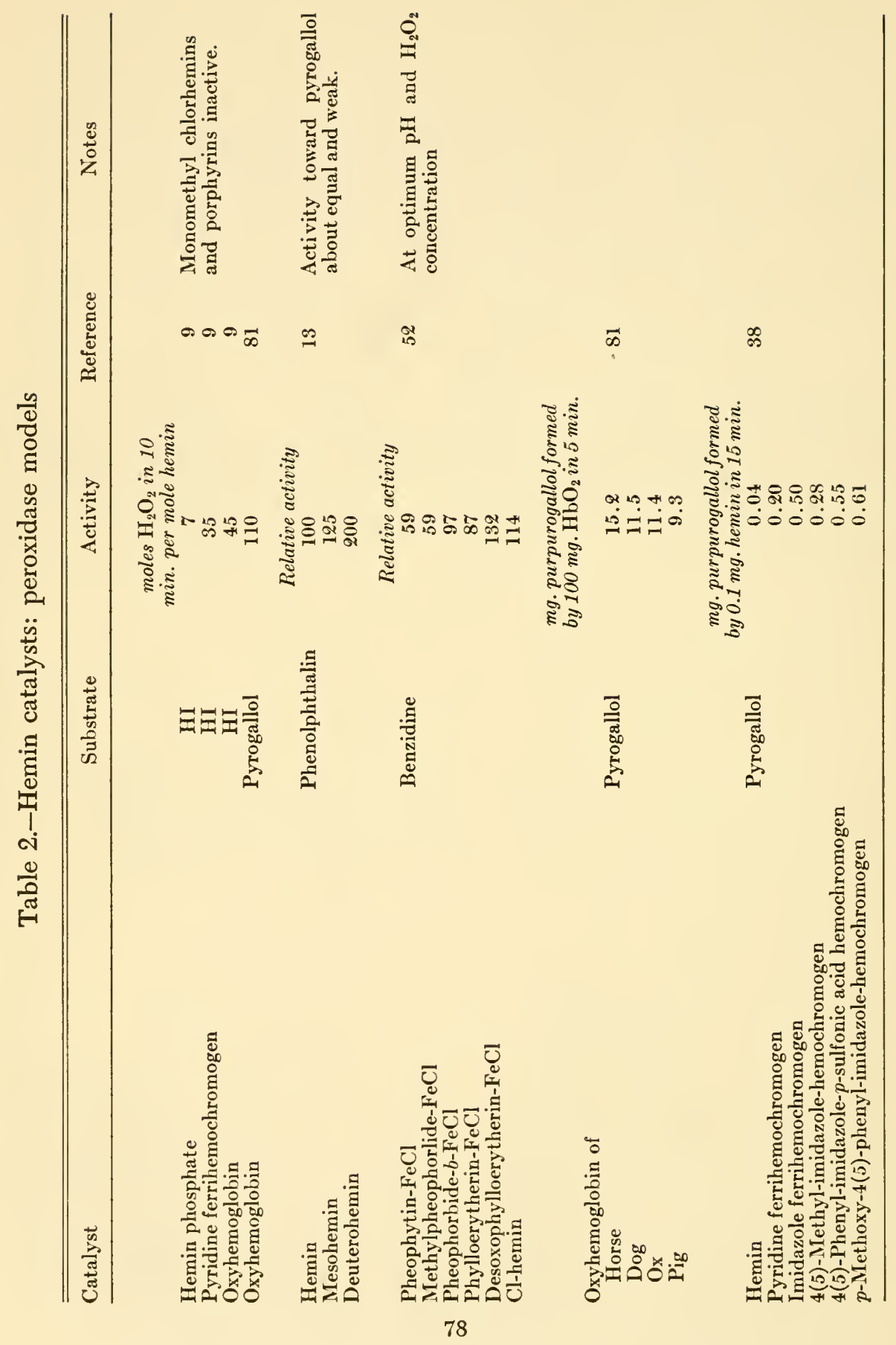


of ordinary charcoal. From hemin, in turn, branch out even more complex systems of still greater catalytic activity, such as the hemochromogens and hemochromogen-charcoal adsorbates, reaching the climax in nature's own products, the hemin enzymes. Upon linking IRON CATALYSTS

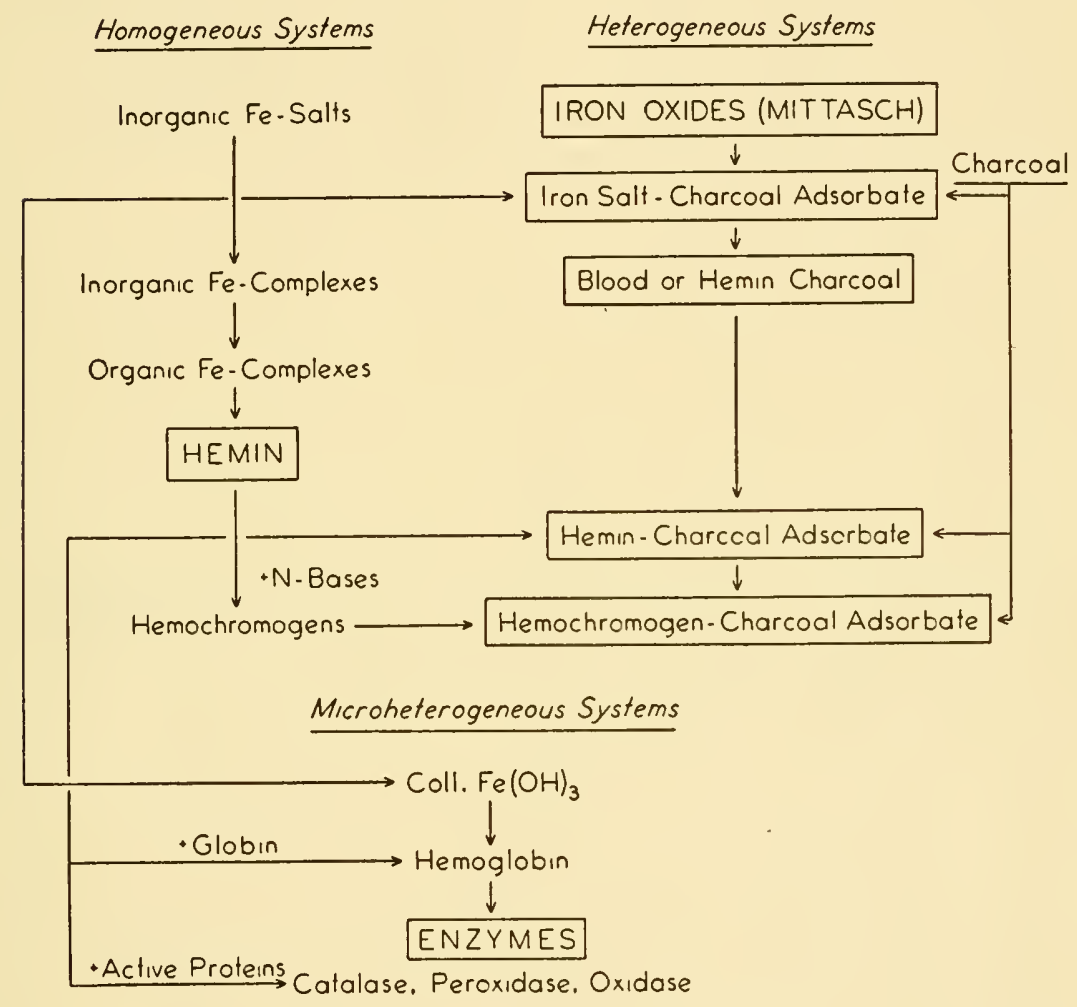

Figure 1.-A family tree of iron catalysts

protoferriheme IX to a specific protein, the enzyme catalase is created which is capable of splitting $10^{5}$ moles of hydrogen peroxide per second under optimum conditions, in spite of the fact that because of the screening of the iron atom, the geometrical requirements for successful collisions with the substrate have become much more stringent (20). This spectacular increase in activity is, however, gained at the price of a decrease in the number of substrates which may be attacked by a given enzyme. Hemin itself shows marked oxidatic, 


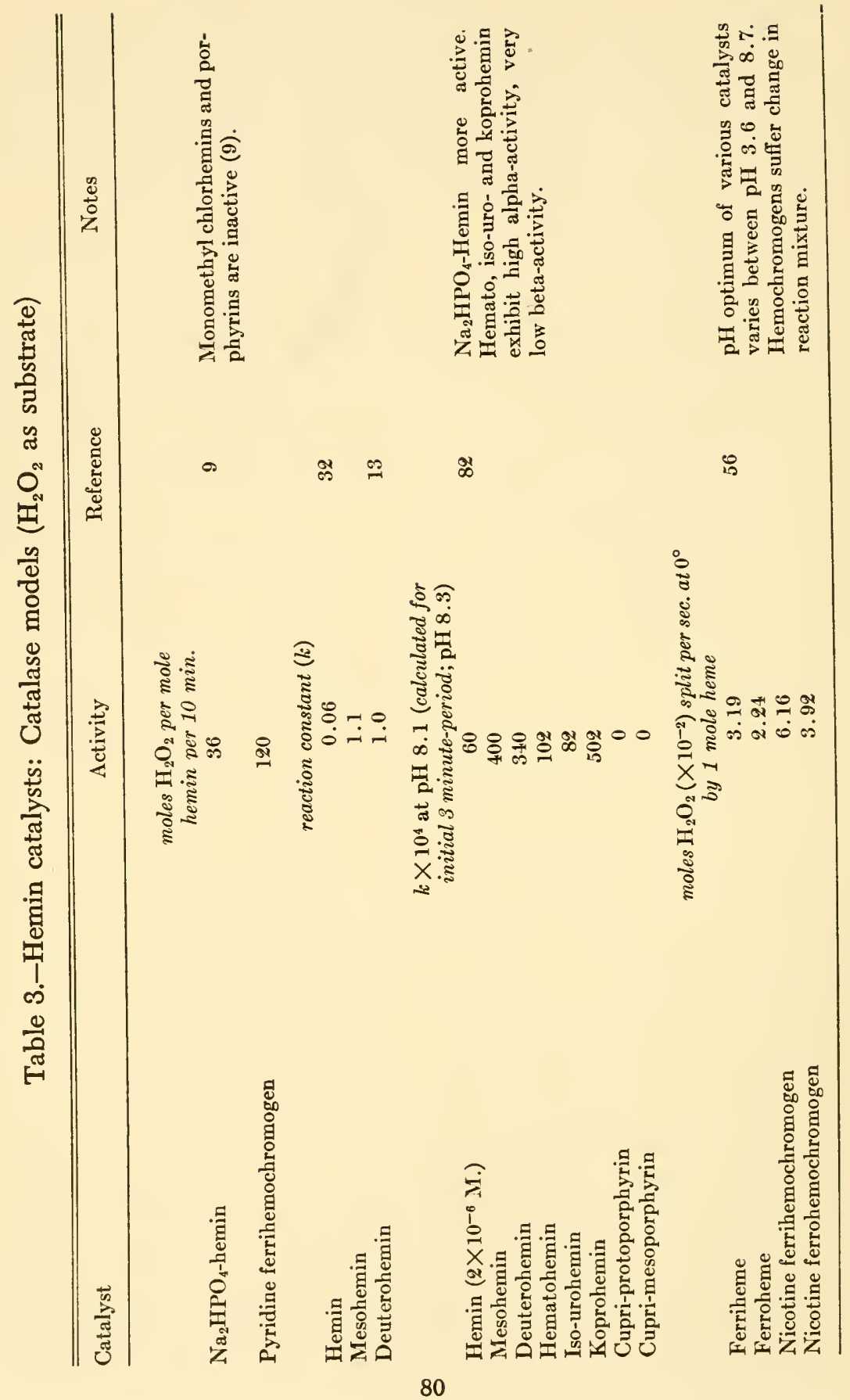




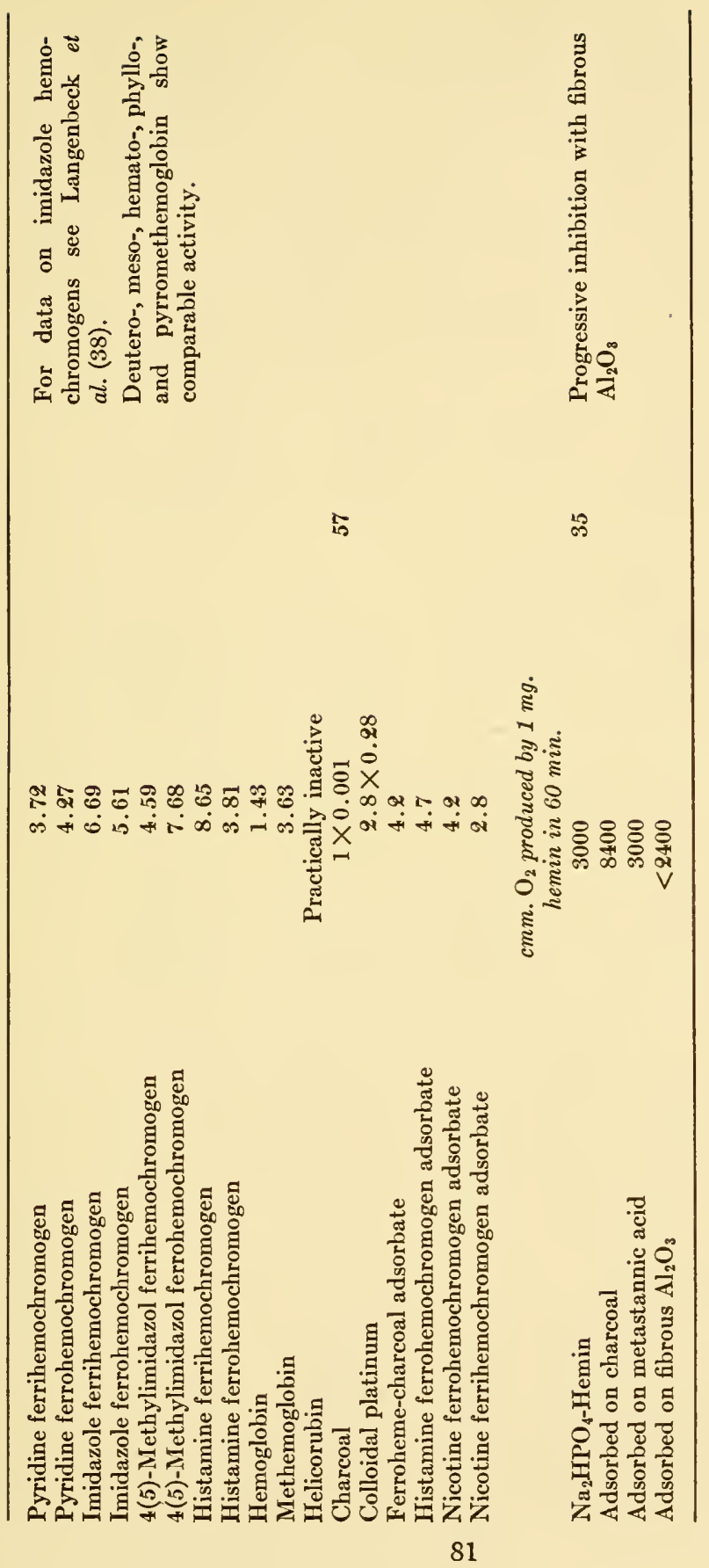




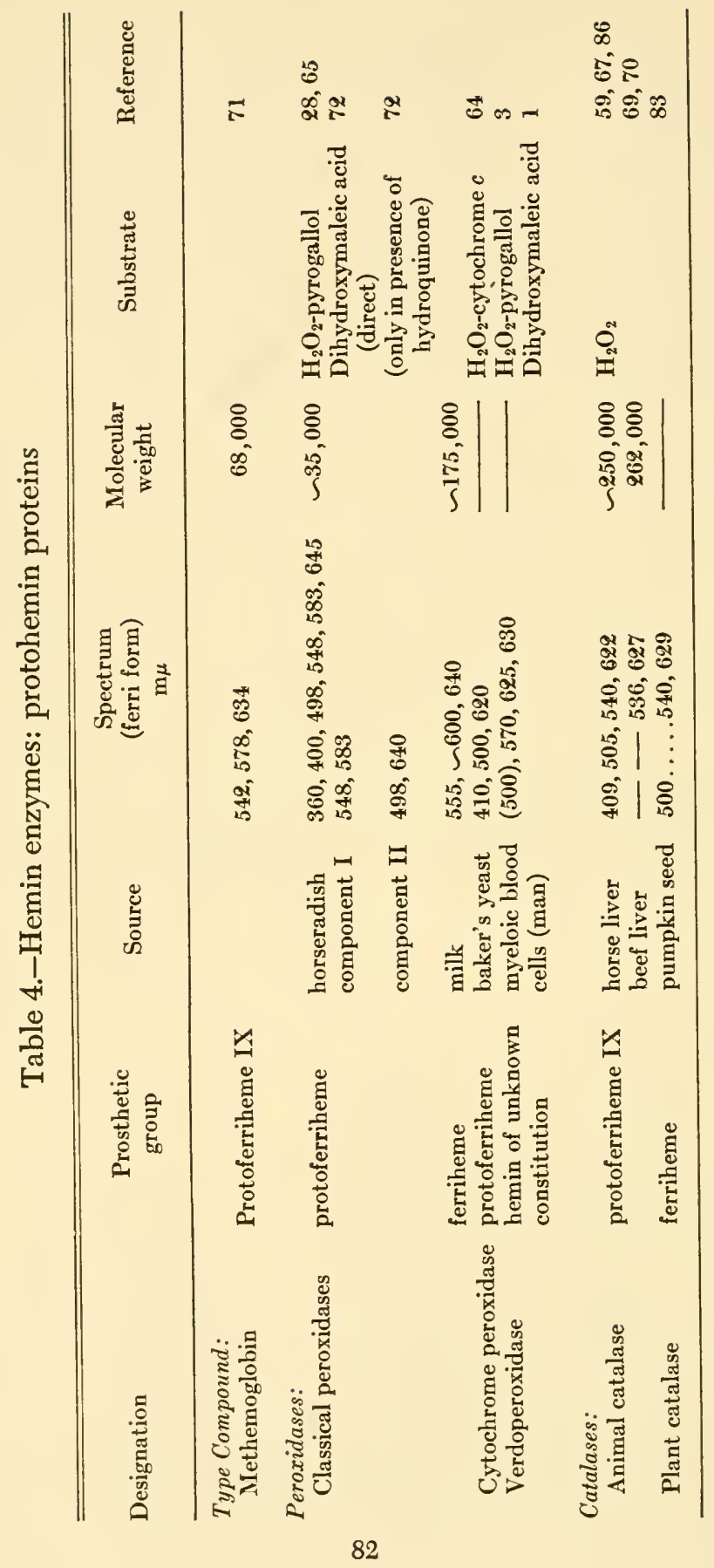




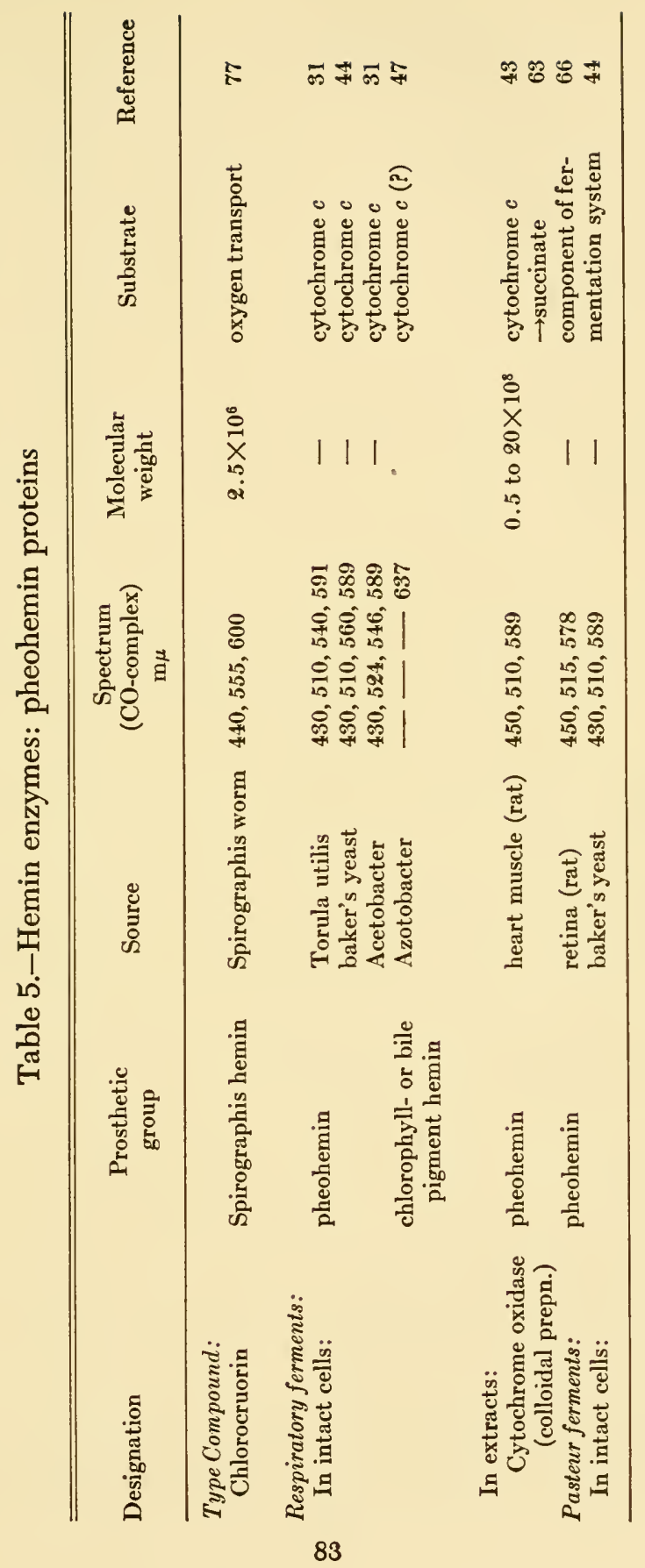


peroxidatic, and catalatic activity. But the enzyme peroxidase has lost the power of splitting hydrogen peroxide without the presence of an oxygen acceptor, and, conversely, the enzyme catalase is apparently devoid of any peroxidatic activity. The faculty of reacting with molecular oxygen, finally, is reserved to a small number of autoxidizable hemin proteins, i.e., the respiratory ferments and the Pasteur enzymes. The ultimate in specialization is reached with hemoglobin, which alone of all iron derivatives and hemochromogens will combine with but will not be oxidized by molecular oxygen. The formally analogous reaction with carbon monoxide is shared by most ferrous complexes, such as ferrous cysteine and ferrohemochromogens of all types. As may be seen from Tables 4 and 5 , at least two different types of hemins occur in hemin enzymes, namely, the red protohemin and mixed-colored pheohemins.

\section{ENZyMe-Substrate Intermediates}

It is difficult to understand why there should be so much controversy over the existence of well-defined intermediates in the course of enzymatic catalyses in general and hemin catalysis in particular. Once it is admitted that enzymes, like all other catalysts, exert their function not by mystical, long-range forces but by actually combining, at some stage of the process, with their substrates and thus creating a new pathway which, although more complex, yields a higher overall rate of reaction, it will depend solely on the lifetime of these intermediates and on their spectroscopic or other properties whether their existence can be detected by experimental means. There are now on record several perfectly clear-cut cases where such enzyme-substrate compounds have been demonstrated by the spectroscope: the intermediate formed in the catalase-ethyl hydrogen peroxide reaction (60), the complexes formed between peroxidase and various proportions of hydrogen peroxide (25), and the less clear-cut observations on complexes between catalase, hydrogen peroxide, and certain inhibitors, such as hydrazine and hydroxylamine (26). The first-named complex exhibits precisely the behavior that the kinetic theories of Michaelis and Henri postulate for an intermediate enzyme-substrate compound: it is unstable and disappears at the rate at which the end products of the reaction are formed. The peroxidase-hydrogen peroxide complexes are also unstable, but their decomposition cannot be due to a true peroxidatic reaction, since it occurs also in the absence of oxygen acceptors. However, the recent work by Karush (25) and Chance (12) on 
rapid reactions affords further proof of the existence of enzymesubstrate complexes as intermediates in the peroxidate catalysis. The nature of the ternary complexes formed by catalase, hydrogen peroxide, and some inhibitors is not yet clear. They may represent ternary complexes between enzyme, substrate, and inhibitor, but they may also be binary enzyme-substrate compounds the lifetime of which is prolonged by the presence of the inhibitors. The interpretation of the phenomena observed in the foregoing enzyme reactions is reinforced considerably by observations made on such closely related, non-enzymatic models as the methemoglobinhydrogen peroxide (21) and methemoglobin-ethyl hydrogen peroxide (58) complexes and the hemin-hydrogen peroxide intermediate (22). If we go back a little, we find that in 1905 Spitalsky observed analogous phenomena in the chromic acid-hydrogen peroxide catalysis. It is safe to conclude that similar intermediates arise in all hemin-catalyzed reactions even if they cannot yet be demonstrated experimentally. The writer has just been informed by Professor Hogness that cytochrome peroxidase, too, forms a typical enzyme-substrate complex with hydrogen peroxide.

The oxidation of ferrous iron to ferric iron by oxygen is usually regarded as a process fundamentally different from the oxygenation of hemoglobin. However, it would simplify matters a good deal if both types of reactions could be brought to a common denominator. In agreement with Haber ${ }^{*}$ and Warburg the reviewer believes that this common link is that in the ferrous-ferric transformation by molecular oxygen an oxygenated intermediate of a structure analogous to oxyhemoglobin is interposed (see Oppenheimer and Stern (49), pp. 14ff.): $\mathrm{Fe}^{++}+\mathrm{O}_{2} \rightarrow \mathrm{Fe}^{++} \mathrm{O}_{2} \rightarrow \mathrm{Fe}^{+++}$.

The reasons for this hypothesis are, first, that molecular oxygen, despite its high potential ( +0.8 volt), appears to be too sluggish an oxidant to react rapidly with ferrous iron without prior activation. It is logical to assume that the hydrogen peroxide, formed as a result of the oxidation of the ferrous iron, arises by interaction of the ferrous-oxygen intermediate with water molecules or hydrogen ions. Secondly, the well-known competition of molecular oxygen and carbon monoxide for the ferrous form of the respiratory ferment, which is governed by Warburg's distribution equations, would be

* This concept appears applicable even to the simplest reactions in the gas phase. Thus Haber and Sachsse (16), on the basis of kinetics experiments, conclude that during the reaction of sodium vapor with oxygen one sodium atom combines with one oxygen molecule. 
difficult to understand if the primary reaction of the enzyme with oxygen were not a reversible equilibrium reaction. This is particularly true for the photochemical dissociation of the iron-carbonyl complex, where it is assumed that, under the influence of light, carbon monoxide is reversibly exchanged with oxygen. All phenomena may be satisfactorily explained on the basis of the assumption that the primary step consists in the formation of a ferrousoxygen intermediate and that the further course of events is governed by the lifetime and reactivity of this complex. In the instance of oxyhemoglobin the intermediate stage is fixed in a unique manner under the influence of the globin component. In that of the oxidases, the intermediate is very short-lived, with the possible exception of the respiratory ferment in baker's yeast (75), where Warburg has observed an absorption band which he tentatively attributes to an oxygen addition compound of the enzyme. A unified theory of oxidase, peroxidase, and catalase action could, then, be based on postulating, with a fair degree of probability, the formation of "moloxides" and "molperoxides," respectively, as the primary process in the catalysis. The mechanism of the oxidation of hemoglobin to methemoglobin by oxygen deviates from this schema because of the high stability of oxyhemoglobin. The well-defined maximum of reaction velocity at low oxygen pressures is in this instance $(10,48)$ largely due to a decrease in the concentration of the free, autoxidizable ferroform as the partial pressure of oxygen in the system is increased. The rate of oxidation is proportional to the concentration of reduced hemoglobin and to a function of the oxygen pressure. In this case oxygen represents both a reactant and an inhibitor, not only because of oxyhemoglobin formation but also, perhaps, through breaking of chain reactions.

\section{On the Mechantcs of Hemin Catalyses}

When considering reactions promoted by hemins, one almost invariably encounters the notion that they must all conform to the pattern of the ferri-ferro cycle. There is no question that this is the most handy explanation: everybody knows that the hemin iron may exist in the reduced $\left(\mathrm{Fe}^{++}\right)$and in the oxidized $\left(\mathrm{Fe}^{+++}\right)$state and that this transformation may be accomplished in a reversible manner even if no bases are linked to the heme. But like many generalizations, this concept as the only explanation is not only hazardous but definitely too narrow.

Let us go back for a moment to the theories that have been ad- 
vanced to explain the catalytic or semi-catalytic effects of iron and other metal ions (37). As in the case of the hemins, we find a widespread inclination to explain everything by a valency change of the metal during catalysis: in the first stage the substrate reduces the trivalent iron (or the bivalent copper) to bivalent iron (or monovalent copper); in the second stage the oxidizing agent, e.g., oxygen or hydrogen peroxide, regenerates the ferric iron or cupric copper, respectively. There are unquestionably instances where this simple hypothesis will serve to explain all the facts, e.g., in the metalcatalyzed oxidation of phenols by oxygen. But in many other instances the hypothesis proves inadequate. Two of the main obstacles are, first, the fact that bivalent iron salts and complexes are so often superior in catalytic activity to the corresponding trivalent compounds and, second, the phenomenon of the "primaerstoss" (alpha-activity). By this we mean the frequent observation that oxidation catalyses, in the presence of ferrous ions, exhibit an initial phase of high velocity which is followed by a steady state of a much lower reaction rate (beta-activity). The transition of ferrous into ferric iron in the course of this process is accompanied by a turnover of many more substrate molecules than would correspond to the number of iron equivalents.

Three different theories have been proposed to explain the facts. All postulate the formation of labile and highly reactive intermediates which are formulated as peroxides by Manchot, as complex compounds by Wieland, and as free radicals by Christiansen, Baeckstroem, and Haber. Manchot assumes that only the bivalent iron is capable of forming peroxides of the type $\mathrm{Fe}_{2} \mathrm{O}_{5}$ or, more recently, of molperoxides of the type $\mathrm{Fe}^{++} \mathrm{H}_{2} \mathrm{O}_{2}$. Such peroxides could oxidize two substrate equivalents and, subsequently, react with hydrogen peroxide present in excess to yield inactive ferric iron, or they could interact with excess hydrogen peroxide to form oxygen while the ferrous iron is regenerated. Wieland, on the other hand, believes that the ferrous iron forms a complex with the substrate or with other substances present in the system with the possible inclusion of the oxidizing agent. By the complex formation the substrate hydrogen is "activated" and is thus made accessible to the attack by the oxidant. The alpha-activity ("primaerstoss") is attributed to a temporary protection of the active ferrous iron contained in the complex against the transition into inactive ferric iron. In this way one ferrous ion is enabled to oxidize a larger number of substrate molecules, the oxidized molecules being released from the complex 
and replaced by fresh substrate molecules. In the well-known chain reaction schemas, as developed by a number of authors, the metal is assumed to initiate the reaction by reacting with a substrate molecule and producing a molecule of high energy content ("energy chains" of Christiansen and of Baeckstroem) or monovalent radicals of high reactivity ("radical chains" of Haber and Franck), whereupon the chain is propagated by such intermediary radicals or "hot" molecules without the further participation of the metal. The chain length, i.e., the yield in product molecules per elementary act initiating the process, is determined by the probability with which two of the intermediary radicals or energy-rich molecules will collide and inactivate each other, and by the presence or absence of specific inhibitors, so-called "chain-breakers." It is important to note that in the metal catalysis of sulfite oxidation, cupric copper and ferrous iron but not ferric iron are able to initiate reaction chains (14). In this connection, the work of Haber and Weiss (17) on the decomposition of hydrogen peroxide by ferrous salt and the nature of the alpha-activity is of particular interest. As is well known, a small amount of ferrous salt, when brought together with a large excess of hydrogen peroxide, is oxidized to ferric salt with the simultaneous liberation of oxygen. The yield depends upon the rate at which the two reactants mix; the ratio $\Delta \mathrm{H}_{2} \mathrm{O}_{2} / \Delta \mathrm{Fe}^{++}$may reach values as high as 15.6 . The underlying chain reaction is formulated by the authors as follows:

$$
\begin{aligned}
& \mathrm{Fe}^{++}+\mathrm{H}_{2} \mathrm{O}_{2}=\mathrm{Fe}^{+++} \mathrm{OH}+\overline{\mathrm{OH}} \\
& \overline{\mathrm{OH}}+\mathrm{H}_{2} \mathrm{O}_{2}=\mathrm{H}_{2} \mathrm{O}+\overline{\mathrm{O}_{2} \mathrm{H}} \\
& \overline{\mathrm{O}_{2} \mathrm{H}}+\mathrm{H}_{2} \mathrm{O}_{2}=\mathrm{O}_{2}+\mathrm{H}_{2} \mathrm{O}+\overline{\mathrm{OH}} \\
& \mathrm{Fe}^{++}+\overline{\mathrm{OH}}=\mathrm{Fe}^{+++} \mathrm{OH}
\end{aligned}
$$

The last equation depicts a chain-breaking reaction. If all the $\mathrm{Fe}^{++}$has been oxidized, the reaction stops. ${ }^{*}$ It is perfectly true that this process, like many reactions studied by Wieland and his students, is not a true catalysis but an induced reaction. But it is like the true catalyses in that small amounts of a promoter or inductor bring about the reaction of a disproportionately large number of substrate molecules. On the other hand, we know that all enzymes are slowly but irreversibly "consumed" during the reactions which they catalyze. Only a finite, although large, amount of protein or hydrogen peroxide can be split by a given quantity of proteinase

- For an analysis of the catalytic decomposition of hydrogen peroxide by ferric salts in acid solution the reader is referred to the papers by Haber and Weiss (18), and Kuhn and Wassermann (36). 
or catalase, respectively. This observation is usually interpreted in terms of an irreversible destruction of the active protein component of the enzyme in some side reaction, e.g., by denaturation or as a result of attack by other enzymes present as impurities. While this may be so, it is not always easy to distinguish clearly between a true catalysis, where the catalyst is progressively eliminated by side reactions, and an induced reaction, where the inductor is slowly converted into an inactive form. This is particularly true of experiments in biological systems. According to all three theories outlined above, the reaction comes to a standstill once all ferrous iron has been converted into ferric iron. A continuation of the process is obviously possible only if the substrate or some other component in the system is able to reduce $\mathrm{Fe}^{+++}$back to $\mathrm{Fe}^{++}$. One could visualize "hybrid" processes where the ferrous iron, in the main reaction, acts as an inductor and where the inactive ferric iron is slowly reduced to the active ferrous form by some "outsider" such as a thiol and is thereby enabled to start the induced reaction all over again. Such a situation, if encountered in living cells, would probably defy any attempt to distinguish between true and apparent catalysis, especially if the reducer is constantly replenished from suitable precursors, e.g., glutathione from protein breakdown.

We go one step further. If iron can break down the potential barrier, shielding stable substrate molecules, either by the ferriferro cycle or by the induced reaction mechanisms just mentioned, is it not possible that iron, when linked up in suitable complexes, could bring about changes in certain substrate molecules or initiate chain reactions without itself suffering a change in valency? It is on this possibility that the issue of the mechanism of catalase action largely hinges. It is well established that the iron in catalase exists in a remarkably stable ferri state. The enzyme is invariably isolated from all sources as the ferri form and it defies reduction with activated hydrogen or with hydrosulfite. This property is unique among hemin proteins; it is not shared even by peroxidase, which may be readily reduced to the ferro form by agents such as sodium hydrosulfite. Moreover, it can be shown that the spectroscopically well-defined intermediate arising in the catalase-ethyl hydrogen

* Theoretically, ferri ion could act catalytically by being reversibly oxidized to a higher stage of valency. Supporting such a view are the spectroscopic observations of Bohnson and Robertson (J. Amer. Chem. Soc., 45, 2493 (1923), on weak acid solutions of ferric salts in the presence of hydrogen peroxide. The color changes occurring in this system were interpreted by these workers to be due to the formation of ferric acid. See, however, Haber and Weiss (18). 
peroxide reaction contains the enzyme in the ferric state (61). Carbon monoxide, which will inhibit many hemin catalyses where ferrous iron is involved, has little or no specific effect on the catalasehydrogen peroxide reaction (61), other reports to the contrary notwithstanding. Some years ago Haber and Willstaetter (19) proposed a chain reaction schema for this catalysis which embodied, as the initial step, a reduction of the enzyme iron to the ferrous form. A little later the present writer modified this schema somewhat (55) with a view to avoiding the necessity of assuming a ferri-ferro cycle. The initial step in that schema consisted in the interaction of two adjacent porphyrin-bound ferri atoms with one hydrogen peroxide atom to yield two monovalent $\mathrm{OH}$-radicals which could then propagate the chain. The iron was assumed to remain in the trivalent form throughout. More recently Keilin and Hartree have advanced a different hypothesis, incorporating the idea of the ferriferro cycle (27):

$$
\begin{array}{ll}
\text { Step I } & 4 \mathrm{Fe}^{+++}+2 \mathrm{H}_{2} \mathrm{O}_{2}=4 \mathrm{Fe}^{++}+4 \mathrm{H}^{+}+2 \mathrm{O}_{2} \\
\text { Step II } & \frac{4 \mathrm{Fe}^{++}+4 \mathrm{H}^{+}+\mathrm{O}_{2}=4 \mathrm{Fe}^{+++}+2 \mathrm{H}_{2} \mathrm{O}}{2 \mathrm{H}_{2} \mathrm{O}_{2}=2 \mathrm{H}_{2} \mathrm{O}+\mathrm{O}_{2}}
\end{array}
$$

The authors state that, in accordance with this schema, the catalysis is greatly inhibited or even suspended in the absence of free oxygen. It will be noted that the hydrogen peroxide is here assigned the role of a specific reducer of the ferri form of catalase, whereas molecular oxygen is considered as the oxidizing agent in the regeneration of the ferri form. The concept of hydrogen peroxide as a reducing agent in itself, although somewhat startling, is not new and finds support in the earlier finding of Kuhn and Wassermann (36) that ferric salts, in the presence of such complex formers as $\alpha, \alpha^{\prime}$-dipyridyl or $o$-phenanthroline, are quantitatively reduced by hydrogen peroxide. But the schema is open to other objections, both on theoretical and on experimental grounds. Thus Johnson and van Schouwenburg (24), Weiss and Weil-Malherbe (80), Sumner and Dounce (cf. 68), and the writer (61) failed to confirm the observation of Keilin and Hartree that catalase is inactive under strictly anaerobic conditions. Furthermore, it seems somewhat strange that, according to the schema of these workers, molecular oxygen should be required for the reoxidation of the ferrous form of catalase in a system containing hydrogen peroxide, which is generally considered a more active oxidizing agent than oxygen, as Dr. M. Gorin has pointed out, in a private communication to the writer. According 
to Dr. Gorin, one would expect, if Keilin's theory were correct, that the reduced form of catalase would accumulate in contact with hydrogen peroxide and in the absence of air. The spectrum of the ferro form of the enzyme, as obtained by treatment with sodium hydrosulfite in the presence of hydrogen sulfide and subsequent removal of the latter, has recently been described by Zeile et al. (87). No spectral change, on the other hand, has as yet been reported for a mixture of catalase and hydrogen peroxide under nitrogen. Attempts to "catch" those ferro-catalase molecules, which might possibly be formed as intermediate products during the catalasehydrogen peroxide reaction, with the aid of carbon monoxide have been unsuccessful (61). The story of the effect of carbon monoxide on catalase under various conditions is just as controversial as the subject of its reaction mechanism (cf. 11, 61, 27). It may suffice here to mention Keilin and Hartree's observations (27) that purified carbon monoxide, in the absence of oxygen, exerts an inhibiting effect on the enzyme which is not relieved by light, whereas certain catalase preparations may be made sensitive to carbon monoxide inhibition in the presence of oxygen by adding traces of azide, cysteine, and glutathione. This effect of carbon monoxide is stated to be completely relieved in a reversible manner by light. The mechanism of this "sensitization" and the reason why crude enzyme preparations are more readily inhibited by carbon monoxide than chemically purified fractions are still obscure. It would seem that, for the present at least, the observations made with the use of carbon monoxide afford no basis for supporting or rejecting Keilin and Hartree's reaction schema. The same is true, in the writer's opinion, of the spectroscopic observations made by Keilin and Hartree (26) on catalase solutions containing sodium azide or hydroxylamine in addition to hydrogen peroxide.

Let us now turn to the theoretical objections raised against Keilin and Hartree's schema. As Weiss and Weil-Malherbe (80) point out, an exclusive reoxidation of ferro-catalase by oxygen would in effect prevent a decomposition of the hydrogen peroxide. For unless a radical chain mechanism is postulated, the oxygen formed by the reduction of the ferri form of the enzyme by hydrogen peroxide (Step I, p. 90) is quantitatively used up again for the reoxidation of a stoichiometric amount of the ferrous form with a simultaneous reduction of the oxygen to hydrogen peroxide. To avoid this difficulty, Keilin and Hartree formulate the oxidation reaction (Step II, p. 90) in such a way that the reduction of oxygen to water does 
not involve the intermediary formation of hydrogen peroxide. This is in opposition to the general views on the mechanism of autoxidation and would appear highly improbable, since it postulates a reaction of a very high order. Furthermore, on the basis of Keilin and Hartree's hypothesis, the reaction, when proceeding in nitrogen, should exhibit the characteristics of an autocatalytic reaction because of the production of increasing amounts of oxygen during the process. This, however, is not indicated in the data of these authors. Sumner and Dounce (cf. 68), who also were unable to confirm Keilin and Hartree's observations with respect to the importance of oxygen for catalase action, prefer the following schema, which is based on earlier ideas of Haber, Euler, and Liebermann:

$$
\begin{array}{ll}
\text { Step I } & \mathrm{Fe}-\mathrm{OH}+\mathrm{H}_{2} \mathrm{O}_{2}=\mathrm{Fe}-\mathrm{OOH}+\mathrm{H}_{2} \mathrm{O} \\
\text { Step II } & \mathrm{Fe}-\mathrm{OOH}+\mathrm{H}_{2} \mathrm{O}_{2}=\mathrm{Fe}-\mathrm{OH}+\mathrm{H}_{2} \mathrm{O}+\mathrm{O}_{2}
\end{array}
$$

In this schema, catalase in its ferric form is represented by the symbol $\mathrm{Fe}-\mathrm{OH}$, which is often employed for methemoglobin, a molecule very similar to catalase. The symbol $\mathrm{Fe}-\mathrm{OOH}$ represents an intermediary catalase peroxide which is assumed to react with a fresh substrate molecule to yield oxygen, water, and the free ferri form of the enzyme. It will be noted that this hypothetical schema, in contrast to that of Keilin and Hartree, does not involve the ferriferro cycle and is in agreement with the spectroscopic observation of a catalase-peroxide complex in the enzyme-ethyl hydrogen peroxide reaction $(58,60)$. But the writer doubts very much whether so simple a schema is adequate to explain all the features of the enzymatic catalysis. We must not forget that the decomposition of hydrogen peroxide can be catalyzed by a variety of agencies besides the enzyme, such as ultraviolet light, dust, metallic and non-metallic surfaces, colloidal platinum, inorganic ferri and ferro salts, cobalti salts, etc. We are therefore confronted with the necessity of finding an explanation for the reaction mechanism which will be equally applicable to these various catalysts. The central theme is, of course, the mode in which hydrogen peroxide is transformed into water and oxygen. The most cogent formulation for this central process has been given by Haber and Weiss (18):

$$
\begin{aligned}
& \overline{\mathrm{OH}}+\mathrm{H}_{2} \mathrm{O}_{2}=\mathrm{H}_{2} \mathrm{O}+\overline{\mathrm{HO}_{2}} \\
& \overline{\mathrm{HO}_{2}}+\mathrm{H}_{2} \mathrm{O}_{2}=\mathrm{O}_{2}+\mathrm{H}_{2} \mathrm{O}+\overrightarrow{\mathrm{OH}}
\end{aligned}
$$

In this schema $\mathrm{OH}$ and $\mathrm{HO}_{2}$ are monovalent radicals. As Haber emphasizes, in a paper published posthumously (18), the progress of 
the reaction through radicals is the main point of the concept, while the propagation of reaction chains through such radicals, although an interesting phenomenon, is of secondary importance. In fact, the two features are not necessarily associated with each other. The detailed study of the kinetics of the decomposition of hydrogen peroxide by ferrous and ferric salts suggests strongly that under certain conditions the radicals may give rise directly to the formation of the end products, water and oxygen, without initiating a chain by further reacting with hydrogen peroxide and thereby reproducing themselves. Thus the catalytic breakdown of hydrogen peroxide by ferri ions in acid medium does not, in general, represent a chain reaction; the oxygen is released here by the process

$$
\mathrm{Fe}^{+++}+\overline{\mathrm{HO}_{2}}=\mathrm{Fe}^{++}+\mathrm{H}^{+}+\mathrm{O}_{2}
$$

Upon slightly changing the experimental conditions, e.g., by increasing the hydrogen peroxide concentration or decreasing the $\mathrm{Fe}^{+++}$, reaction 2 is favored, with the result that chains appear (18).

The second question relates to the way in which the central process, consisting of steps 1 and 2 , is initiated by the various catalytic agents. The radicals $\mathrm{OH}$ and/or $\mathrm{HO}_{2}$ can be created only by a monovalent attack on hydrogen peroxide. It is the monovalent character of the primary reaction of the catalyst with the substrate which, according to Haber and Weiss $(18,78)$, is the common feature of the chemical, photochemical, and electrochemical primary processes. Thus the radicals may arise by the transformation of a metal ion into the one of next higher valency, as in the instance of ferrous ions,

$$
\mathrm{Fe}^{++}+\mathrm{H}_{2} \mathrm{O}_{2}=\mathrm{Fe}^{+++}+\mathrm{OH}^{-}+\overline{\mathrm{OH}}
$$

or by the reduction of a higher valent state, as in the case of ferric ions:

$$
\mathrm{Fe}^{+++}+\mathrm{HO}_{2}^{-}=\mathrm{Fe}^{++}+\overline{\mathrm{HO}_{2}}
$$

Hydrogen peroxide may also be split into two $\mathrm{OH}$ radicals by ultraviolet light:

$$
\mathrm{H}_{2} \mathrm{O}_{2}+\mathrm{hv}=2 \overline{\mathrm{OH}}
$$

In heterogeneous systems, involving metal surfaces, the radical $\mathrm{OH}$ is believed to result from a simple electron transfer,

$$
\mathrm{H}_{2} \mathrm{O}_{2}+\text { electron }(\text { meta } 1)=\mathrm{OH}^{-}+\overline{\mathrm{OH}}
$$

While reaction 7 is an expression of the oxidizing action of $\mathrm{H}_{2} \mathrm{O}_{2}$, the reducing properties of the molecule are attributed to its anion $\left(\mathrm{HO}_{2}^{-}\right)$, in accordance with the equation 


$$
\mathrm{HO}_{2}{ }^{-}=\overline{\mathrm{HO}_{2}}+\text { electron }
$$

Both these functions are related by the dissociation equilibrium

$$
\mathrm{H}_{2} \mathrm{O}_{2} \rightleftarrows \mathrm{H}^{+}+\mathrm{HO}_{2}^{-} \text {(dissociation constant, } \mathrm{K} \text { ) }
$$

It follows that there is no fundamental difference between the homogeneous and heterogeneous reactions with regard to the elementary process. Weiss (79) has recently discussed the mechanism of catalase and peroxidase action from the same point of view. In his presentation of the central reaction, step 2 is replaced by the equation

$$
\mathrm{O}_{2}^{-}+\mathrm{H}_{2} \mathrm{O}_{2}=\mathrm{OH}^{-}+\overline{\mathrm{OH}}+\mathrm{O}_{2}
$$

where $\mathrm{O}_{2}{ }^{-}$has been substituted for $\mathrm{HO}_{2}$ on the basis of new evidence. These two radicals are related to each other by the electrolytic dissociation equilibrium

$$
\mathrm{O}_{2}^{-}+\mathrm{H}^{+} \rightleftarrows \overline{\mathrm{HO}}_{2} \text { (dissociation constant, } \mathrm{K}_{\mathrm{HO}_{2}} \text { ). }
$$

Weiss treats the catalase-hydrogen peroxide reaction as a heterogeneous catalysis and as an analogon to the catalytic decomposition of the same substrate by colloidal platinum, which has been so carefully studied by Bredig. The similarity is accentuated by the fact that neither catalase nor colloidal platinum shows peroxidase activity toward acceptors of the type of iodide ion or pyrogallol. In both instances the primary process is formulated as a surface reaction involving the radicals $\mathrm{OH}$ and $\mathrm{HO}_{2}$. The author assumes that the iron atom of the enzyme is alternatively reduced to the ferrous and reoxidized to the ferric form by the hydrogen peroxide during the course of the catalysis. The role of the metal atom in the porphyrin skeleton is regarded as that of facilitating rapid electron transfers, since the valency change of the iron in the porphyrin ring system takes place without appreciable dislocation of heavy particles, in contrast to the situation in the instance of free ferrous and ferric ions, where the water dipoles in the hydration shell must undergo rearrangements upon a change in the charge of the central atom. Furthermore, he believes that the system of conjugated double bonds surrounding the iron in the heme group makes for a rapid "conduction" of the inner electron by virtue of their loosely held $n$-electrons.

In analogy to the action of colloidal platinum on hydrogen peroxide, as formulated by the same author, the mechanism of catalase action is depicted as follows: 


$$
\begin{aligned}
& \mathrm{Fe}^{+++}+\mathrm{HO}_{2}^{-} \rightleftarrows \mathrm{Fe}^{+\imath}+\overline{\mathrm{HO}}_{2} \\
& \mathrm{Fe}^{++}+\mathrm{H}_{2} \mathrm{O}_{2}=\mathrm{Fe}^{+++}+\mathrm{OH}^{-}+\overline{\mathrm{OH}} \\
& \mathrm{Fe}^{++}+\overline{\mathrm{OH}}=\mathrm{Fe}^{+++}+\mathrm{OH}^{-}
\end{aligned}
$$

The production of molecular oxygen is attributed to reactions 1 and $2 \mathrm{a}$.

It will be noted that the process, although involving free radicals, is not a conventional chain reaction, since the enzyme iron plays an active role in three stages: 10,11 , and 12 . This is partly based on the finding that in the model system platinum-hydrogen peroxide an average chain length of only about five links may be assumed. It is therefore considered probable that in the enzymatic catalysis, under the usual experimental conditions, the chain reaction practically degenerates to a simple radical reaction. It will be recalled that one of the criticisms directed against the chain reaction theory of Haber and Willstaetter by Haldane was that one would expect the rate to be proportional to the square root of the enzyme concentration rather than to the enzyme concentration itself, as is actually the case. By postulating very short chains, as Weiss does, the feature of the proportionality between reaction velocity, enzyme concentration, and substrate concentration is retained without sacrificing the essential concept of Haber, i.e., the postulate of intermediate, monovalent radical formation. The further objection of Haldane that the assumption of the same type of radicals $(\mathrm{OH}$ and $\mathrm{HO}_{2}$ ) in various kinds of enzyme reactions, as was done by Haber and Willstaetter, was in conflict with the well-known specificity of the oxidizing enzymes, is also met if very short chains are assumed. In this case the radicals are present only in so low a concentration that their oxidizing action on acceptors, e.g., iodide ion or oxyphenols, remains below the threshold of sensitivity of the analytical methods. All these considerations refer to the "normal" course of the catalase reaction, i.e., to conditions where the enzyme and the substrate concentration are within the range usually employed in kinetic studies and activity determinations. There can be little doubt that the explosive type of hydrogen peroxide decomposition, such as is produced in concentrated peroxide solutions when a relatively large amount of enzyme is added, represents a chain process with a long chain length. There may even be branched chains, such as are assumed to occur during "knocking" in internal combustion engines, when the relatively slow combustion along a flame front of regular rate of progression changes over into detonation (cf. Lewis and van Elbe, 40). 
It is not feasible, within the space allotted to this discussion, to cite all the experimental evidence and theoretical arguments in favor of the intermediary formation of free radicals or of the chain reaction character of the hydrogen peroxide catalysis or of oxidative enzyme action in general. It must suffice, therefore, to refer to the original publications where the existence of $\mathrm{OH}$ radicals during hydrogen peroxide photolysis (73a) or the formation of the $\mathrm{HO}_{2}$ in reactions with molecular oxygen (78a) have been observed. The experiments of Schwab et al. (53a) on the effect of typical chainbreakers on the catalase reaction did not yield conclusive results. But the experiments of Barron (5) on the effect of antioxidants on the rate of oxidation of unsaturated fatty acids by hemochromogens are very suggestive. The pioneer work of Michaelis on the radical nature of the semiquinones, which arise through monovalent reduction or oxidation of reversible dyestuffs makes it all but imperative to admit the intermediary existence of monovalent radicals (monohydropyridine, monohydroalloxazine, monohydrothiamine, etc.) during cellular respiration, unless reactions of high order are postulated.

Those who are interested in the manner in which the action of other oxidative enzymes, e.g., peroxidase or dehydrogenases, may be interpreted in terms of radical chain mechanisms are referred to the papers by Haber and Willstaetter (19), Weiss (79), and the review article by Moelwyn-Hughes (45).

\section{Autoxidizable Iron Compounds}

We return to the starting point of the discussion: What makes the iron atom and the oxygen molecule "click" during the primary process? Why are some iron compounds autoxidizable and others not? No satisfactory answer may at present be given to this question, which certainly attracts the attention of many workers in the field of biological oxidation. Only partial solutions have been offered to this key problem.

Ferrous iron is relatively stable in acid solution and is rapidly oxidized to ferric iron by molecular oxygen in alkaline solution. The state of the metal under these two conditions obviously differs in one respect. In the acid medium the iron is present as ferrous ion, whereas in alkaline solution it exists as non-ionized ferrous hydroxide. Smythe (54), in an interesting paper, points out that the oxidation of ferrous ion involves the separation of a negative charge 
(electron) from a nucleus which already carries two positive charges. Non-ionized ferrous hydroxide, on the other hand, should be more easily oxidized, since this process represents only the separation of the electron from an electroneutral substance. If this reasoning is sound, one would expect that ferrous iron, if built into a non-ionized compound, should be readily autoxidizable regardless of the acidity of the medium. The rate of autoxidation of ferrous complexes would then be expected to be a function of the $\mathrm{pH}$ only in so far as the stability of the complex is affected by changes in hydrion concentration. In other words, under any given condition the velocity of the reaction with oxygen should be proportional to the concentration of the ferrous complex, which in turn is a function of the concentrations of the ferrous ions and the complex-forming anions. Since the latter, within a given $\mathrm{pH}$ range, depends on the hydrion concentration, one would expect that within this range the rate of oxidation would vary with the $\mathrm{pH}$. Smythe proceeded to test this working hypothesis on a series of inorganic and organic iron complexes of relatively simple configuration. His manometric study of the rate of autoxidation of ferrous pyrophosphate and ferrous metaphosphate, which are stable over a wide $\mathrm{pH}$ range, bore out the prediction. The reaction rate varied strongly with the hydrion concentration, decreasing markedly in both instances with an increase in acidity. The case of ferrocyanide is of special interest because its structure bears a certain resemblance to the core of iron porphyrin complexes. In both instances the iron atom is surrounded by four groups, containing nitrogen linked to carbon atoms. In the case of the ferrocyanide these groups carry negative charges. To escape from the metal atom, an electron must pierce this "negative atmosphere," which is obviously difficult. We are not surprised, therefore, to find that the rate of autoxidation of ferrocyanide is extremely small in spite of the fact that the iron is present in covalent rather than in ionic linkage. If it were possible to reduce the negative charge on the groups encircling the iron atom, its reaction with oxygen should be facilitated. Indeed we find that if the charge is reduced by replacing one of the $\mathrm{CN}^{-}$groups by ammonia, a compound, pentacyanoammine-ferroate, is formed which is attacked by oxygen at an appreciable rate. The striking observation of Baudisch and Davidsohn (7) that the oxygen uptake is more rapid at $\mathrm{pH} 2$ than at 7 or 12 , in contrast to the findings obtained with iron pyro- or metaphosphate, is explained by Smythe in terms of a suppression of the ionization under the influence of the increased hydrion concen- 
tration and a consequent decrease of the negative charges around the iron atom.

However, Smythe's views are not shared by Weiss (78). This author believes, in direct contradiction to the hypothesis that only coordinatively bound ferrous iron is autoxidizable, that only "free" ferrous ions react, in general, with molecular oxygen. By "free" ions Weiss means iron with incompletely filled electron orbits. The autoxidation of ferrous sulfate is formulated in accordance with Weiss's theory of simple electron transfers as follows:

$$
\begin{aligned}
& \mathrm{Fe}^{++}+\mathrm{O}_{2} \rightarrow \mathrm{Fe}^{+++}+\mathrm{O}_{2-}^{-}, \quad \mathrm{O}_{2}^{-}+\mathrm{H}^{+} \rightleftarrows \overline{\mathrm{HO}_{2}} \\
& \mathrm{Fe}^{+++}+\mathrm{O}_{2}^{-} \rightarrow \mathrm{Fe}^{++}+\mathrm{O}_{2} \\
& \mathrm{Fe}^{++}+\overline{\mathrm{HO}_{2}} \rightarrow \mathrm{Fe}^{++}+\mathrm{HO}_{2}^{-}, \quad \mathrm{HO}_{2}^{-}+\mathrm{H}^{+}=\mathrm{H}_{2} \mathrm{O}_{2}
\end{aligned}
$$

Equation 15 explains the formation of hydrogen peroxide as an end product of autoxidation processes. If, however, it is not caught in statu nascendi, e.g., by cerium hydroxide, the hydrogen peroxide is relatively rapidly decomposed by reaction with ferrous ironferric ions and hydroxyl ions or water being formed as final products. During the entire process the oxygen molecule is stepwise reduced by four electrons with the formation of four hydroxyl ions.

The sequence given above, according to Weiss, provides a logical explanation for the slow rate at which an acidified ferrous sulfate solution is autoxidized. The latter does not indicate that the interaction between the ferrous ions and oxygen (reaction 13) is slow but, on the contrary, that the rate of the back reaction 14 , which involves a reduction of ferric ions, is quantitatively significant. Under stationary conditions and for a given partial tension of oxygen, the rate of reaction 15 is defined by the ratio of the velocities of the partial reactions 13 and 14 ; in other words, by the ratio $\left[\mathrm{Fe}^{++}\right]$to $\left[\mathrm{Fe}^{+++}\right]$. The greater this ratio the more positive will be the oxidation-reduction potential and the higher will be the rate of ferrous salt oxidation. The essential feature in reactions of this type, according to Weiss, is not the formation of stable ferrous iron complexes but the fact that the ferric ions are protected against reduction by the formation of still more stable ferric iron complexes. Thus, in instances where the ferrous complex is more stable than the corresponding ferric complex, as in the case of ferrous tridipyridyl sulfate, no autoxidation takes place.

A decidedly more mechanistic explanation is offered by Theorell (73) for the failure of cytochrome $c$ to react with molecular oxygen in the physiological $\mathrm{pH}$ range: "The heme of the cytochrome is ... built into the protein component in a manifold way: by means 
of thioether bindings from the side chains of the porphyrin to the protein, and by means of two histidine-imidazole groups strongly bound to $\mathrm{Fe}$ on each side of the flat heme disc. Thus the heme group appears to be built into a crevice in the protein molecule. This explains why cytochrome $c$ is not autoxidizable, since oxygen can never approach the iron atom, and why no CO-compounds or cyanide compounds are formed at physiological pH values."

\section{Oxygen Transfer in Living Cells}

In every aerobically living cell we find a number of hemin proteins, e.g., a respiratory ferment, three different cytochromes, catalase and/or peroxidase. There is also frequently present what Keilin calls the "unspecific cell hematin." More recently another functional type of hemin enzymes has been found in such cells, which cata-

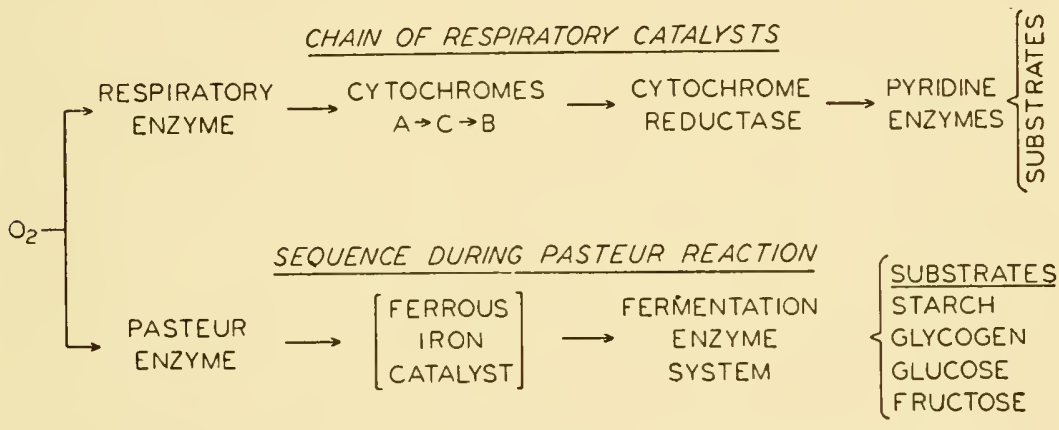

Figure 2.-Function of molecular oxygen in respiration and Pasteur reaction

lyzes the inhibiting effect of oxygen on fermentation and glycolysis (Pasteur reaction). Some data pertaining to these various iron porphyrin proteins are given in Tables 4 and 5 .

Two groups of these intracellular hemin proteins are endowed with the power to react directly with molecular oxygen: the oxygentransferring enzymes of respiration (for short, "Warburg enzymes") and the aerobic fermentation-inhibiting catalysts (for short, "Pasteur enzymes"). Inasmuch as the Pasteur reaction is the subject of another paper, it must suffice here to present only the particular working hypothesis which the writer is advancing on the basis of recent work in this laboratory. See Figure 2.

Photochemical work $(44,61)$ has revealed the pheohemin nature of the prosthetic group of the Pasteur enzymes in rat retina and in 
baker's yeast. It is thus seen that catalysts of the same type, although differing in details, have been developed by nature to keep in check both alcoholic and lactic acid fermentation in the presence of oxygen. As Figure 2 indicates, oxygen is "mobilized" for its two chief tasks in aerobic cell life by two autoxidizable hemin proteins (Warburg and Pasteur enzymes) acting in analogous manner but independently of each other. The effect of a variation of the oxygen tension (and, for that matter, of carbon monoxide concentration in inhibitor experiments) on the overall phenomena of cellular respiration and aerobic fermentation will depend on the affinity which the iron contained in the two types of enzymes has for these gases. Just as is the case with the respiratory transport protein hemoglobin in various vertebrate species, there will be variations in the gasdissociation curves for these enzymes from cell to cell. Depending on whether a particular Pasteur catalyst has a higher or a lower affinity for oxygen or carbon monoxide than the Warburg ferment in the same cell, we will expect to find respiration more or less readily affected by a lowering of oxygen tension or a given ratio of carbon monoxide to oxygen than the Pasteur reaction in that cell. If these gases do not vary in their effect on the two processes, as Warren (unpublished observations) found to be true in the case of bone marrow, it must be because the affinity of the enzymes for the gases is equal. In general it would appear that in higher animal tissues the Warburg ferment has a greater affinity for oxygen and a lesser affinity for carbon monoxide than the Pasteur enzyme in the same tissue (Laser effect, 39); in unicellular systems (certain bacteria, human myeolocytes) the reverse seems to be true (Kempner effect, 29). It would appear that there are as many (slightly) different Warburg and Pasteur enzymes as there are living forms. Probably the difference resides in the protein rather than in the hemin group of the molecules, except in the case of Azotobacter, which seems to have a Warburg enzyme with a green hemin rather than a mixedcolored or pheohemin in the prosthetic group (47). The reasons for interposing a hypothetical ferrous iron catalyst between the Pasteur enzyme and the fermentation system, as indicated in the figure, cannot be explained here because of lack of space.

In closing, some model experiments may be mentioned which are being carried out at present in this laboratory. Some years ago, Lipmann (41) showed that a Pasteur effect in cell-free systems (yeast and muscle fermentation extracts) may be produced by the addition of suitable reversible dyestuffs of sufficiently high potential (see 
Figure 3). If our concept of the mechanism of the Pasteur reaction is correct, it should be possible to replace the dyestuffs in Lipmann's experiments by an autoxidizable hemochromogen of suitable potential. Pyridine, histidine, and picoline hemochromogen have been tried without positive results. The experiments with the very positive systems nicotine and nicotinic acid amide hemochromogen, on
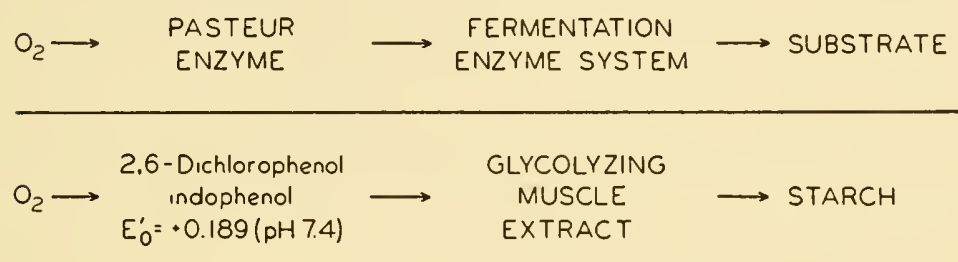

$\mathrm{O}_{2} \longrightarrow \begin{gathered}\text { Naphthol-Sulfonate } \\ \text { indophenol } \\ E_{0}^{\prime}=+0147 \mathrm{v} .(\mathrm{pH} 6.6)\end{gathered} \longrightarrow \begin{gathered}\text { FERMENTING } \\ \text { YEAST MACERATION } \\ \text { EXTRACT }\end{gathered} \longrightarrow\left\{\begin{array}{c}\text { GLUCOSE } \\ \text { +Hexose Diphosphate } \\ \text { SUCROSE }\end{array}\right.$

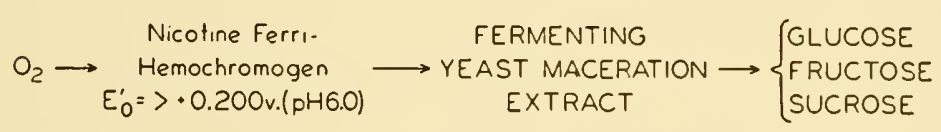

Figure 3.-Attempts at reconstruction of Pasteur reaction in cell-free systems

the other hand, have yielded some encouraging results which, unfortunately, did not prove to be reproducible at will. One has the impression that the underlying idea is correct but that some of the factors involved in the complex reaction have thus far evaded adequate control.

The problem of the Pasteur reaction, as the writer sees it, is now shifting from the nature of the catalyst to the nature of its substrate.

\section{REFERENCES}

1. Agner, K., Act. Physiol. Scand., Vol. II. Suppl. 8 (1941).

2. Ahlström, L., and v. Euler, H., Z. physiol. Chem., 200, 233 (1931).

3. Altschul, A. M., Abrams, R., and Hogness, T. R., J. Biol. Chem., 136, 777 (1940); and unpublished observations.

4. Barron, E. S. G., Physiol. Rev., 19, 184 (1939).

5. Barron, E. S. G., and Lyaran, C. M., J. Biol. Chem., 123, 229 (1937).

6. Barron, E. S. G., de Meio, R. H., and Klemperer, F., J. Biol. Chem., 112,125 (1935).

7. Baudisch, O., and Davidsohn, D., J. Biol. Chem., 71, 501 (1927).

8. Bergel, F., and Bolz, K., Z. physiol. Chem., 215, 25 (1933).

9. Brann, L., Thesis, Zürich, 1927.

10. Brooks, J., Proc. Roy. Soc. (London), B 109, 35 (1931); 118, 560 (1935). 
11. Califano, L., Naturwissenschaften, 22, 249 (1934).

12. Chance, B., J. Biol. Chem., 140, xxiv (1941).

13. v. Euler, H., Runehjela, D., and Steffenburg, Sv., Arkiv Kemi, Mineral. Geol., Abt. B, 10, 1 (1929).

v. Euler, H., Nilsson, H., and Runehjelm, D., Sved. Kem. Tidskr., 41 , 85 (1929).

v. Euler, H., and Jansson, B., Monatsh. Chem., 53-54, 1014 (1929).

14. Franck, J., and Haber, F., Sitz. Ber. Preuss. Akad. Wiss. Berlin, 1931, p. 250.

15. Franke, W., Liebigs Ann., 498, 129 (1932).

16. Haber, F., and Sachisse, H., Z. physik. Chem., Bodenstein Festschrift, 1931, p. 831.

17. Haber, F., and Weiss, J., Naturwissenschaften, 20, 948 (1932).

18. Haber, F., and Weiss, J., Proc. Roy. Soc. (London), A 147, 332 (1934).

19. Haber, F., and Willstaetter, R., Ber., 64, 2844 (1931).

20. Haldane, J. B. S., Proc. Roy. Soc. (London), B 108, 559 (1931).

21. Havrowitz, F., Z. physiol. Chem., 232, 159 (1935).

22. Haunowitz, F., Enzymologia, 4, 139 (1937).

23. Heubner, W., Naturwissenschaften, 16, 515 (1928).

24. Johnson, F. H., and van Schouwenbung, K. L., Nature, 144, 634 (1939).

25. Karush, F., J. Biol. Chem., 140, lxvi (1941).

26. Keilin, D., and Hartree, E. F., Proc. Roy. Soc. (London), B 121, 173 (1936).

27. Keilin, D., and Hartree, E. F., Proc. Roy. Soc. (London), B 124, 397 (1938).

28. Keilin, D., and Mann, T., Proc. Roy. Soc. (London), B 122, 119 (1937).

29. Kempner, W., J. Cell. Comp. Physiol., 10, 339 (1937); Am. J. Tuberculosis, 2, 157 (1939).

Kempner, W., and Gaffron, M., Am. J. Physiol., 126, 553 (1939).

30. Krebs, H. A., Biochem. Z., 193, 347 (1928).

31. Kubowitz, F., and HaAs, E., Biochem. Z., 255, 347 (1932).

32. Kuhn, R., Brann, L., Seyffert, C., and Furter, M., Ber., 60, 1151 (1927).

33. Kuhn, R., and Meyer, K., Naturwissenschaften, 16, 1028 (1928).

Meyer, K., J. Biol. Chem., 103, 25 (1933).

34. Kuhn, R., and Meyer, K., Z. physiol. Chem., 185, 193 (1929).

35. Kuhn, R., and Wassermann, A., Ber., 61, 1550 (1928).

36. Kuhn, R., and Wassermann, A., Leibigs Ann., 503, 203 (1933).

37. Langenbeck, W., Die Organischen Katalysatoren (Berlin, 1935).

38. Langenbeck, W., Hutschenreuter, R., and Rottic, W., Ber., 65, 1750 (1932).

39. Laser, H., Biochem. J., 31, 1671, 1677 (1937).

40. Lewis, B., and van Elbe, G., Combustion, Flames and Explosions of Gases (Cambridge, 1938).

41. Lipmann, F., Biochem. Z., 265, 133 (1933); 268, 205 (1934).

42. Lyman, C. M., and Barron, E. S. G., J. Biol. Chem., 121, 275 (1937).

43. Melnick, J. L., Science, 94, 118 (1941).

44. Melnick, J. L., J. Biol. Chem., 141, 269 (1941).

45. Moenwyn-Hughes, E. A., Ergebnisse d. Enzymforschung, 6, 23 (1937).

46. Negelein, E., Biochem. Z., 243, 386 (1941).

47. Negelein, E., and Gerischer, W., Biochem. Z., 268, 1 (1934).

48. Neill, J., and Hastings, A. B., J. Biol. Chem., 63, 479 (1925).

49. Oppenheimer, C., and Stern, K. G., Biological Oxidation (The Hague, 1939).

50. Reid, A., Ergebnisse d. Enzymforschung, 1, 325 (1932).

51. Rem, A., Z. Angew. Chem., 47, 515 (1934). 
52. Reuter, F., Willstaedt, H., and Zirm, K. L., Biochem. Z., 261, 353 (1933).

53. Robinson, M. E., Biochem. J., 18, 255 (1934).

53a. Schwab, G. M., Rosenfeld, B., and Rudolph, L., Ber. Deutsch. Chem. Ges., 66, 661 (1933).

54. Sмythe, C. V., J. Biol. Chem., 90, 251 (1931).

55. Stern, K. G., Z. physiol. Chem., 209, 176 (1932).

56. Stern, K. G., Z. physiol. Chem., 215, 35 (1933).

57. Stern, K. G., Z. physiol. Chem., 219, 105 (1933).

58. Stern, K. G., Nature, 136, 335 ( 1935 ).

59. Stern, K. G., J. Biol. Chem., 112, 661 (1936).

60. Stern, K. G., J. Biol. Chem., 114, 473 (1936); Enzymologia, 4, 145 (1937).

61. Stern, K. G., J. Gen. Physiol., 20, 631 (1937).

62. Stenn, K. G., Yale J. Biol. and Med., 10, 161 (1937).

63. Stern, K. G., Cold Spring Harbor Symposia on Quantitative Biology, 7, 312 (1939).

64. Stern, K. G., and Gordon, W., unpublished observations.

65. Stern, K. G., and Kegeles, G., unpublished.

66. Stern, K. G., and Melnick, J. L., J. Biol. Chem., 139, 301 ( 1941 ).

67. Stern, K. G., and Wyckoff, R. W. G., J. Biol. Chem., 124, 573 (1938).

68. Sumner, J. B., Advances in Enzymology, 1, 163 (1941).

69. Sumner, J. B., and Dounce, A. L., J. Biol. Chem., 121, 417 (1937).

70. Sumner, J. B., and Gralen, N., J. Biol. Chem., 125, 33 (1938).

71. Svedberg, Trr., J. Biol. Chem., 103, 311 (1933).

72. Theorell, H., Arkiv Kemi, Mineral. Geol., B 14, No. 20 (1941).

73. Theorell, H., J. Am. Chem. Soc., 63, 1820 ( 1941 ).

73a. Urey, H. C., Dawsey, L. H., and Rice, F. O., J. Am. Chem. Soc., 51, 1371 (1929).

TAylor, H. S., and Gould, A., J. Am. Chem. Soc., 55, 859 (1933).

74. Warburg, O., Ergebnisse d. Enzymforschung, 7, 210 ( 1938 ).

75. Warburg, O., and HaAs, E., Naturwissenschaften, 22, 207 (1934).

76. Warburg, O., and Negelein, E., Biochem. Z., 200, 414 (1928).

77. Warburg, O., and Negelein, E., Biochem. Z., 244, 9 ( 1932 ).

78. Weiss, - J., Naturwissenschaften, 23, 64 (1935).

78a. Weiss, J., Trans. Faraday Soc., 31, 688 (1935).

79. Weiss, J., J. Phys. Chem., 41, 1107 ( 1937 ).

80. Weiss, J., and Weil-Malmerbe, H., Nature, 144, 866 (1939).

81. Willstaetter, R., and Pollinger, A., Z. physiol. Chem., 130, 281 (1923).

82. Zerre, K., Z. physiol. Chem., 189, 127 (1930).

83. ZeiLe, K., Z. physiol. Chem., 195, 39 (1931).

84. Zeile, K., Ergebnisse d. Physiol., 35, 498 ( 1933 ).

85. Zeile, K., in Oppenheimer Handbuch d. Biochemie, Ergaenzungswerk, 1, 708 (1933) (Jena).

86. Zeile, K., and Hellstroem, H., Z. physiol. Chem., 192, 171 ( 1930 ).

Stern, K. G., J. Biol. Chem., 121, 561 (1937).

87. Zeile, K., Fawaz, G., and Ellis, V., Z. physiol. Chem., 263, 181 (1940). 


\title{
Nicotinamide Nucleotide Enzymes
}

\author{
FRITZ SCHLENK \\ School of Medicine, University of Texas
}

$R_{\text {EPORTs of Investigations in the field of the nicotinamide nu- }}^{\text {cleotide enzymes are now so numerous as to make impossible }}$ a complete review of the subject here; and in any case it would seem to be unnecessary in view of the comprehensive articles that have been published in recent years (la-k). Emphasis will therefore be laid upon those details that have not been extensively discussed in previous articles and to report some recent advances.

\section{CODEHYdROGENASE I AND II}

Of the two coenzymes in this group, codehydrogenase I (cozymase, diphosphopyridine nucleotide, Co I), was detected in 1906 by Harden as a coenzyme of alcoholic fermentation (2). The other, codehydrogenase II (triphosphopyridine nucleotide, Co II), was discovered in 1932 by Warburg (3). In a series of investigations by the von Euler school (1921-34) methods for the purification and determination of cozymase were elaborated. Its classification as a nucleotide was ascertained, its codehydrogenase nature was propounded, and the numerous reports denying its existence were refuted (le,f). In 1934 the most noteworthy discovery in the field was made: Warburg and Christian isolated nicotinamide from codehydrogenase II (4) and demonstrated its function as part of a hydrogen-transporting coenzyme (5). In 1935 nicotinamide was isolated also from cozymase (6), and soon the very close relationship between these two coenzymes was established by the work of the institutes in Berlin and Stockholm.

Both coenzymes are nicotinamide-adenine dinucleotides, the only difference between them being that codehydrogenase II contains three molecules, and codehydrogenase I two molecules, of phosphoric acid. Later the transformation of one coenzyme into the other was achieved by enzymatic dephosphorylation of codehydrogenase II and enzymatic as well as chemical phosphorylation of codehydrogenase I (7). The yield of codehydrogenase II by this reaction is, however, rather low; the isolation from red blood cells is still the 
only method that has been described for preparing it in the pure state.

Codehydrogenase II occurs in much lower concentration than codehydrogenase $\mathrm{I}$. In Table 1 are given some typical examples of the occurrence of the two coenzymes. The values are only approximate because crude extracts were used for the determinations. With improved methods of determination it probably will be necessary to revise these values considerably, especially those for codehydrogenase II.

Table 1.-Some examples of the occurrence of the codehydrogenases I and II

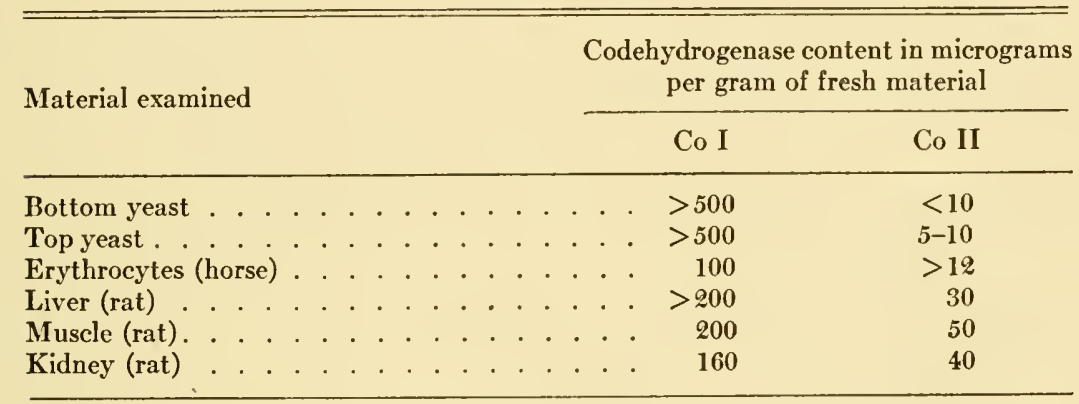

It is remarkable that despite the low content of codehydrogenase II in the source material and the difficulty and lengthiness of the isolation procedure, the preparation of codehydrogenase II in a pure state, the isolation of nicotinamide therefrom, and the demonstration of its mode of action were carried out by Warburg and Christian in a remarkably short time. This work greatly facilitated the isolation of nicotinamide from cozymase and the subsequent work on this coenzyme in the Stockholm institute.

In Tables 2 and 3 the methods of isolating the two compounds are shown schematically. The procedures are different in the two cases, but typical steps of purification known from earlier work in nucleotide chemistry are involved in both instances. Significant steps, some of them new, in the preparation of codehydrogenase II are the following: removal of proteins by acetone; fractionation of the crude mixture of nucleotides as barium salts, yielding adenosine polyphosphate and coenzyme I as by-products; solution of the coenzyme in methanol-hydrochloric acid and reprecipitation by ethylacetate $(5,8)$. 
The method that led to the isolation of codehydrogenase I in a pure state is based in the initial stage on the purification steps recommended by Myrbäck (1e). A definite improvement is the precipitation of cozymase by cuprous chloride dissolved in a concentrated Table 2.-Preparation of codehydrogenase II from horse erythrocytes

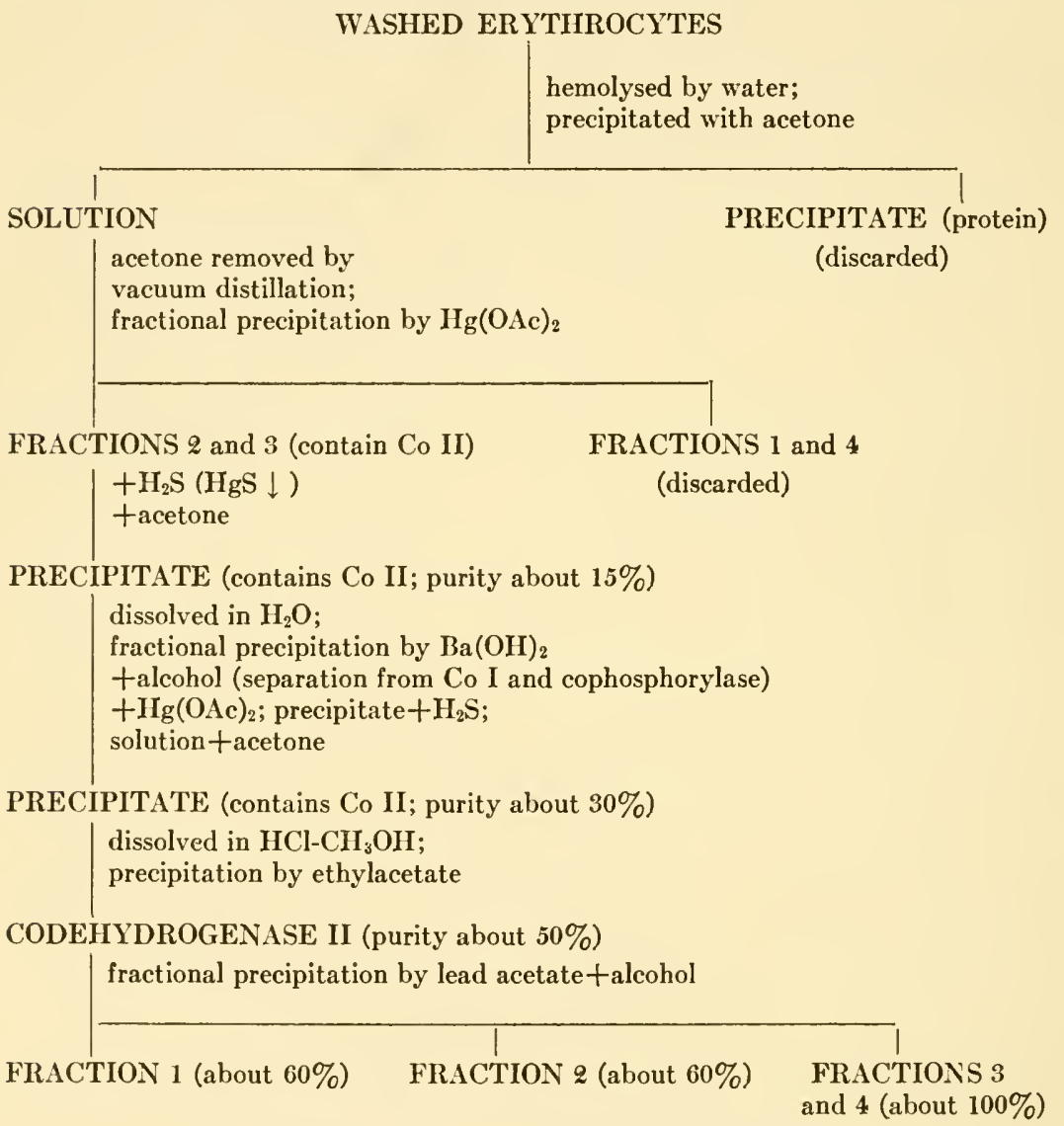

solution of potassium chloride (9). This procedure has been employed in all preparative methods subsequently recommended (10-13). Since pure cozymase does not give a stable precipitate with the cuprous chloride reagent, such precipitation cannot be repeated. Apparently some impurity in the crude cozymase solutions plays an important role in producing a stable precipitate. Decomposition of the precipitate by hydrogen sulfide involves a considerable loss of 
Table 3.-Preparation of codehydrogenase I from yeast

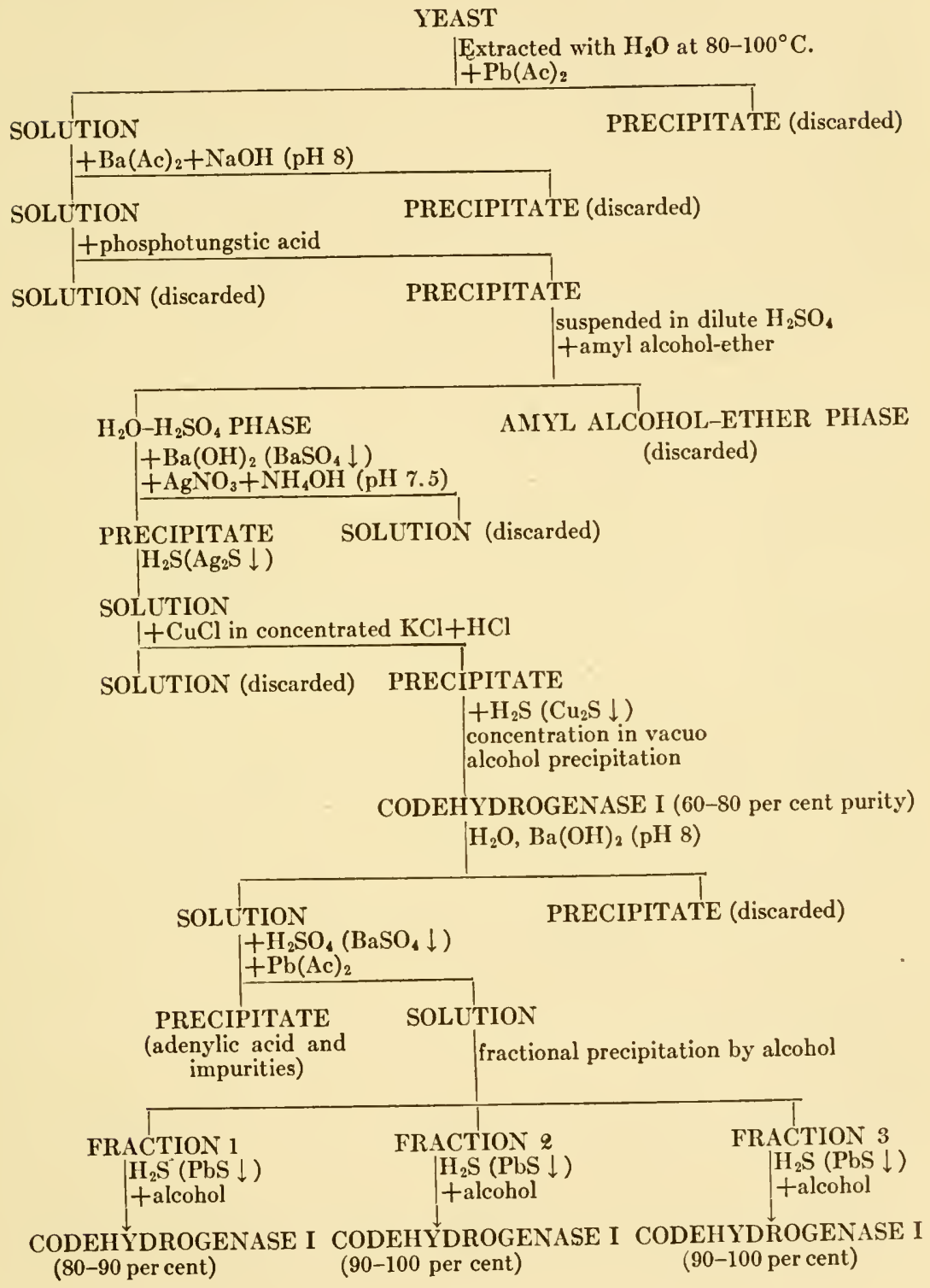


the coenzyme by adsorption on the copper sulfide. For desorption the sulfide precipitate is aerated until slight oxidation of copper sulfide is attained. This method of desorption, the details of which were described some years ago $(9,14)$, has also proved advantageous in preventing losses by adsorption on silver sulfide. The final purification is brought about by fractional barium and lead precipitation. Both codehydrogenases prepared according to the methods given in Tables 2 and 3 contain traces of impurities which complicate their use in the spectrographic methods employed in dehydrogenase investigations. The great stability toward oxidizing agents (le) permits the destruction of impurities by treatment with bromine water

Table 4.-Properties of codehydrogenases I and II

\begin{tabular}{|c|c|c|c|c|}
\hline Property & Codehydrogenase I & Ref. & Codehydrogenase II & Ref. \\
\hline $\begin{array}{l}\text { Empirical formula } \\
\text { Molecular weight }\end{array}$ & $\begin{array}{c}\mathrm{C}_{21} \mathrm{H}_{27} \mathrm{O}_{14} \mathrm{~N}_{7} \mathrm{P}_{2} \\
663\end{array}$ & 16 & $\begin{array}{r}\mathrm{C}_{21} \mathrm{H}_{23} \mathrm{O}_{17} \mathrm{~N}_{7} \mathrm{P}_{3} \\
743\end{array}$ & 5 \\
\hline Structural units & $\begin{array}{l}1 \text { Mol. nicotinamide } \\
1 \text { Mol. adenine } \\
2 \text { Mol. pentose } \\
2 \text { Mol. phosphoric acid }\end{array}$ & $\begin{array}{r}6 \\
17\end{array}$ & $\begin{array}{l}1 \text { Mol. nicotinamide } \\
1 \text { Mol. adenine } \\
2 \text { Mol. pentose } \\
3 \text { Mol. phosphoric acid }\end{array}$ & $\begin{array}{l}5 \\
5\end{array}$ \\
\hline Base equivalent & 1 & 18 & $3-4(?)$ & $\begin{array}{r}5 \\
19\end{array}$ \\
\hline $\begin{array}{l}\text { Stability: } \\
\text { Oxidized form }\end{array}$ & & & & \\
\hline $\begin{array}{c}\text { In } 0.1 \mathrm{~N} \mathrm{HCl} \text { at } \\
100^{\circ} \mathrm{C} .\end{array}$ & $\begin{array}{l}50 \% \text { destroyed after } \\
8 \text { min. }\end{array}$ & 20 & $\begin{array}{l}50 \% \text { destroyed after } \\
7.3 \text { min. }\end{array}$ & 4 \\
\hline In $0.1 \mathrm{~N} \mathrm{NaOH}$ & $\begin{array}{l}50 \% \text { destroyed after } \\
17 \text { min. }\left(20^{\circ}\right)\end{array}$ & 20 & $\begin{array}{l}50 \% \text { destroyed after } \\
12 \text { min. }\left(23^{\circ}\right)\end{array}$ & 4 \\
\hline Reduced form & & & & \\
\hline In $0.1 \mathrm{~N} \mathrm{HCl}$ at $20^{\circ} \mathrm{C}$. & $\begin{array}{l}\text { activity disappears } \\
\text { immediately }\end{array}$ & $\begin{array}{l}20 \\
10\end{array}$ & $\begin{array}{l}\text { activity disappears } \\
\text { immediately }\end{array}$ & 5 \\
\hline $\begin{array}{l}\text { In } 0.1 \mathrm{~N} \mathrm{NaOH} \text { at } \\
100^{\circ} \mathrm{C} \text {. }\end{array}$ & $\begin{array}{l}\text { slight decrease in activ- } \\
\text { ity after } 10 \mathrm{~min} \text {. }\end{array}$ & 21 & & \\
\hline $\begin{array}{l}\text { In } 0.1 \mathrm{~N} \mathrm{NaOH} \text { at } \\
\quad 20^{\circ} \mathrm{C} \text {. } \\
\text { Absorption spectrum: } \\
\text { Oxidized form }\end{array}$ & stable & 21 & stable & 5 \\
\hline Maximum at $260 \mathrm{~m} \mu$ & $\mathrm{E}=3.8 \times 10^{7}\left[\frac{\mathrm{cm}^{2}}{\mathrm{Mol}}\right.$ & $\begin{array}{r}1 \mathrm{i} \\
5 \\
8\end{array}$ & $\mathrm{E}=3.5 \times 10^{7}\left[\frac{\mathrm{cm}^{2}}{\mathrm{Mol}}\right.$. & $\begin{array}{l}5 \\
8\end{array}$ \\
\hline Reduced form & & 10 & & \\
\hline Maximum at $260 \mathrm{~m} \mu$ & $\mathrm{E}=3.3 \times 10^{7}\left[\frac{\mathrm{cm}^{2}}{\mathrm{Mol}}\right.$ & 22 & $\mathrm{E}=3.1 \times 10^{7}\left[\frac{\mathrm{cm}^{2}}{\mathrm{Mol}}\right.$ & 8 \\
\hline $340 \mathrm{~m} \mu$ & $\mathrm{E}=1.1 \times 10^{7}\left[\frac{\mathrm{cm}^{2}}{\mathrm{Mol}}\right.$ & 22 & $\mathrm{E}=1.0 \times 10^{7}\left[\frac{\mathrm{cm}^{2}}{\mathrm{Mol} .}\right.$ & 8 \\
\hline
\end{tabular}


(8). Filtration through a column of activated alumina has also been employed successfully (15).

Recently some modifications in the preparation of cozymase as given in Table 3 have been described (10-13). The improvements consist mainly in omitting some of the purification steps, which can be done without complications if a good quality of yeast is used as the source material. B. J. Jandorf has introduced the adsorption of cozymase on charcoal in his method of preparation (13). S. Ochoa has developed a method in which muscle tissue is used as source material (11), and P. Ohlmeyer has described a method for the preparation of dihydrocozymase (10). From 10 kilograms of yeast one gram of almost pure, or 0.5 gram of pure codehydrogenase $I$ is obtained. The yield of codehydrogenase II from 1000 liters of erythrocytes is about 2.5 grams of almost pure, or 1.0 gram of absolutely pure preparation. The coenzymes precipitated from aqueous solution by organic solvents are not crystalline. Table 4 shows the composition and properties of codehydrogenase I and II.

The most important part of the work on the structure of the coenzymes has been concerned with the nicotinamide moiety, its mode of action, and the linkage between nicotinamide and the rest of the molecule. This work was begun by Warburg (8) and continued by Karrer and his co-workers (23a-f). Warburg showed first that in codehydrogenase II the nicotinamide reacts with two atoms of hydrogen in the presence of substrate and apoenzyme, forming a dihydro compound. This compound can also be obtained by reduction with hydrosulfite in a slightly alkaline medium. The reduced coenzyme has an absorption maximum at $340 \mathrm{~m} \mu$, whereas the maximum at $260 \mathrm{~m} \mu$. has lost some of its intensity by the reduction (see Figure 1). By reoxidation the original state is restored.

Catalytic hydrogenation yields an uptake of six hydrogen atoms by the oxidized coenzymes and of four hydrogen atoms by the biologically reduced coenzymes. Experiments with adenine and its derivatives showed that under the same conditions these compounds are very slowly reduced by catalytic hydrogenation, whereas free nicotinamide exhibits the same properties as the coenzymes upon catalytic reduction (5). It should be remembered that the catalytic reduction which leads to the hexahydro compounds is irreversible, and the products obtained are inactive as coenzymes. These experiments demonstrated that the place of the reversible (biological) reduction-i.e., the center of the coenzyme activity-is the nicotinamide nucleus. 


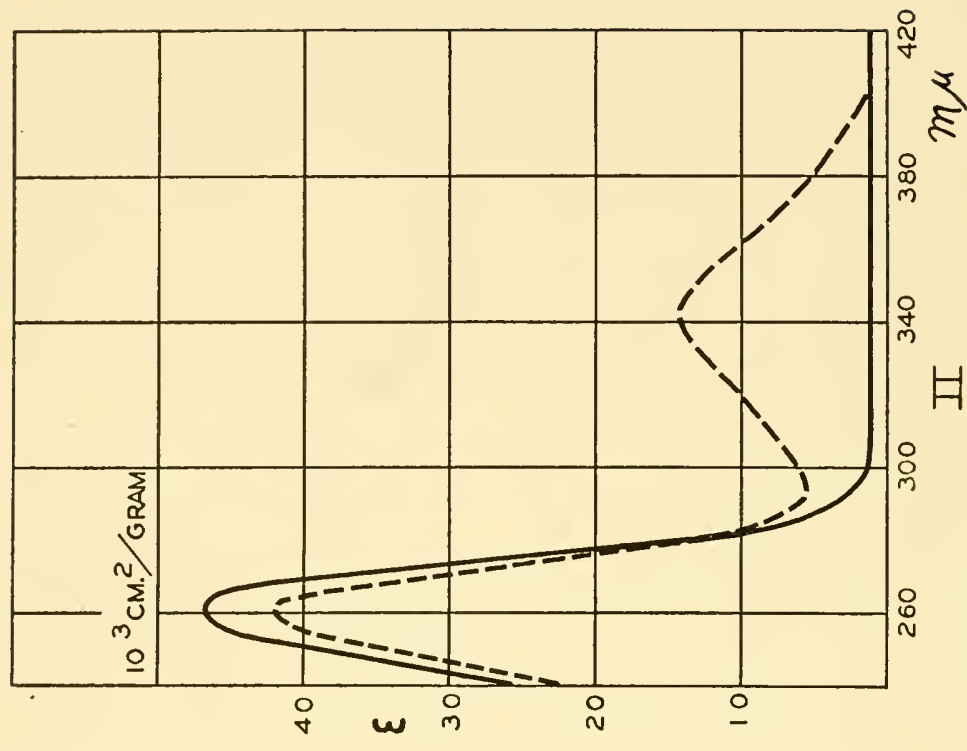

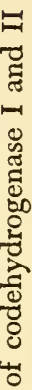

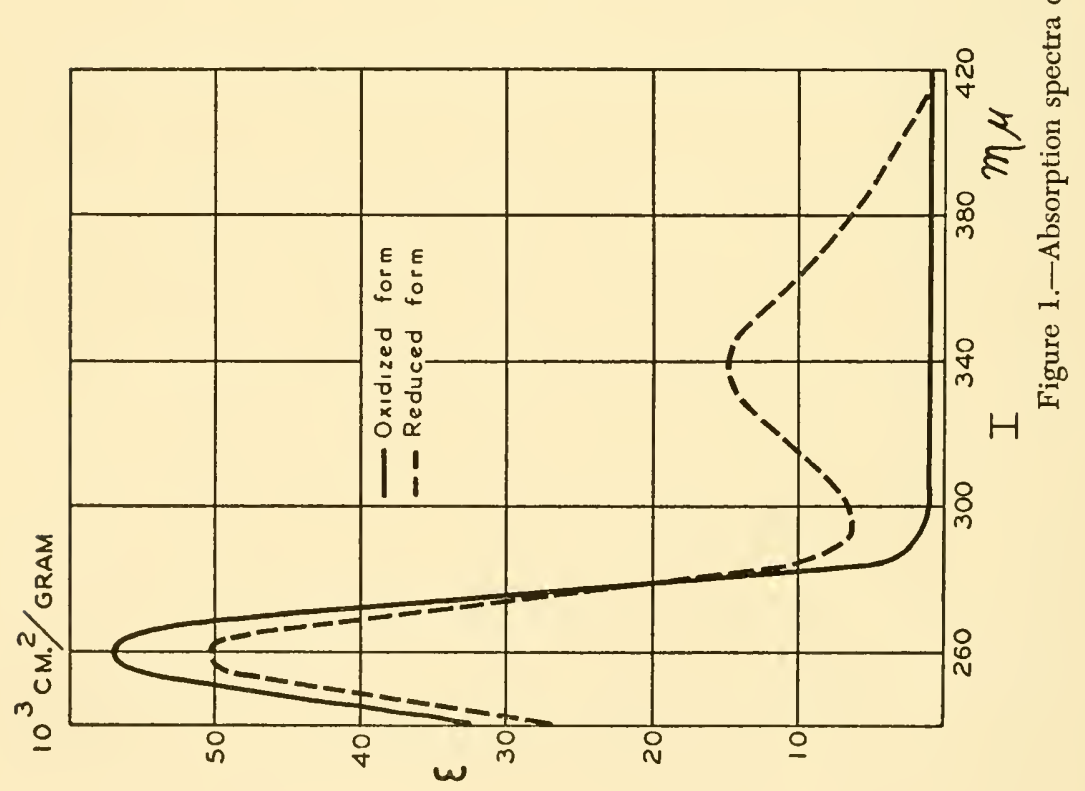


The next problem was to determine what type of linkage existed between nicotinamide and the rest of the molecule and what changes were brought about in the ultraviolet absorption by reduction of the coenzymes to the dihydro form. This question was answered by the extensive experiments of Karrer and his co-workers. In the first place, the three functional centers of the nicotinamide molecule had to be taken into consideration. "Model compounds" were therefore prepared which were substituted in these positions with simple organic groups. Among these compounds may be mentioned the following typical representatives: nicotinamide iodomethylate (I), nicotinic acid ethylimido ether (II), and monomethyl nicotinamide (III).

\section{FORMULA 1}

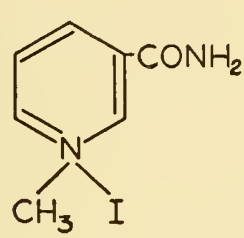

(I)

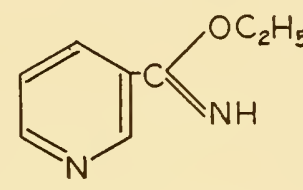

(II)<smiles>CNC(=O)c1cccnc1</smiles>

(III)

Of these compounds only the nicotinamide iodomethylate exhibited properties similar to the coenzymes. Like the coenzymes it is reducible by hydrosulfite (see formula 2 ), and the absorption maximum of its dihydro product is $360 \mathrm{~m} \mu$ (340 m $\mu$ is the typical absorption maximum of the dihydrocoenzymes), which disappears when the solution is acidified, as does that of the dihydrocoenzymes.

FORMULA 2
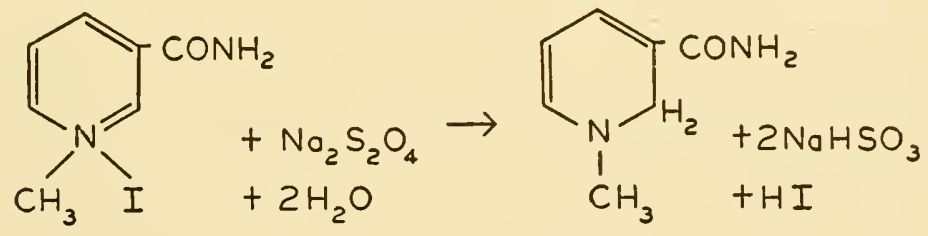

These results indicated strongly that in the codehydrogenases the nicotinamide is bound as a quaternary pyridinium base. The experimental data accumulated in testing this working hypothesis soon established its validity. It was already known that quaternary pyri- 
dinium bases are readily subject to reduction processes. Concerning the position where the reduction takes place, the following possibilities had to be considered.

\section{FORMULA 3}

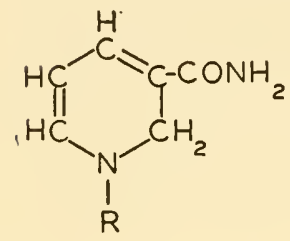

(a)

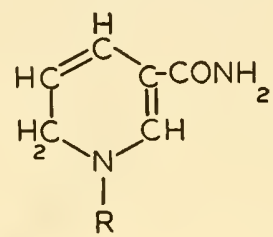

(b)

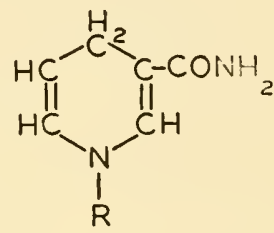

(c)

A comparison of the earlier known compounds of this group showed noteworthy differences between the $p$-dihydro compounds and the reduced coenzymes, but very good agreement between the latter and the model $o$-dihydro compounds. Whether the reduction takes place according to formula $3 \mathrm{a}$ or $3 \mathrm{~b}$ could not be decided $(23 a, c)$.

The next step was the preparation of nicotinamide derivatives which are substituted by carbohydrate radicals on the ring nitrogen atom. The properties of these compounds showed a still better correspondence with those of the coenzymes. The best representative of these compounds which can be prepared in a pure state was found to be tetra-acetyl-glucosido-nicotinamide bromide. The absorption maximum of its dihydro derivative is very similar to that of the reduced codehydrogenases. In addition to similarity in optical properties the model nucleosides share with the coenzymes the sensitivity of the glycosidic linkage toward alkali when in the oxidized form, and stability toward alkali when reduced; also, the action of strong acid on the dihydro compounds yields, according to Karrer, products which no longer have the absorption band at $340 \mathrm{~m} \mu$. Furthermore, both the model compounds and the coenzymes are very sensitive toward hypoiodite, which destroys the pyridine ring (24).

When the pyridinium model compounds are reduced to the dihydro compounds, the ring nitrogen of the reaction products is trivalent, and they do not contain the acid group. Corresponding to this acid group in the model compounds is the phosphoric acid in the coenzymes. Upon reduction an acid group is liberated according to the following scheme $(8,21)$ : 
FORMULA 4
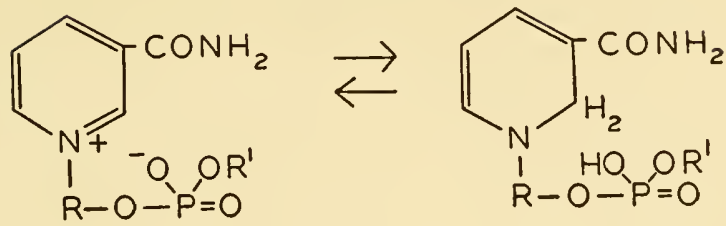

According to Haas this reaction can be controlled manometrically if bicarbonate is present in the medium (25). The attempts of Karrer to synthesize nicotinamide derivatives with pentose as a substituent were not successful. Attempts with arabinose and xylose gave oily products which could not be obtained in a pure nor in a crystalline form. The closest link between the model compounds and the coenzymes, therefore, was missing. The compound consisting of nicotinamide and pentose has been obtained from cozymase (26). It possesses all the expected properties and has some biological interest. Since no extensive publication on this subject has yet appeared, the preparation and properties of the compound are here discussed in somewhat more detail.

As has been pointed out, the splitting of the linkage between nicotinamide and pentose is the first result of acid as well as of alkaline hydrolysis of cozymase. Therefore only enzymatic splitting could yield this very labile nucleoside. For this purpose the nucleotidase discovered by Bredereck in sweet almond press-cake was chosen (27). After purification this enzyme has the following properties, which make it suitable for the preparation of the nucleoside: The $\mathrm{pH}$ for optimum activity is about the same as that for the optimum stability of cozymase. The preparations are free from

Table 5.-Enzymatic splitting of cozymase

\begin{tabular}{ccccc}
\hline $\begin{array}{c}\text { Time of } \\
\text { hydrolysis, } \\
\text { in hours }\end{array}$ & $\begin{array}{c}\text { Fermentation } \\
\text { test, per- } \\
\text { centage Co I } \\
\text { found }\end{array}$ & $\begin{array}{c}\text { Percentage } \\
\text { phosphorus } \\
\text { split off }\end{array}$ & $\begin{array}{c}\text { Percentage } \\
\text { nicotinamide } \\
\text { split off }\end{array}$ & $\begin{array}{c}\text { Spectro- } \\
\text { photometric } \\
\text { determination; } \\
\text { percentage } \\
\text { "pyridinium } \\
\text { compound" }\end{array}$ \\
\hline 0 & 100 & 0 & 0 & 100 \\
24 & 51 & 48 & & \\
$96^{*}$ & 17 & 85 & $<10$ & 92 \\
120 & $<1$ & 100 & & \\
\hline
\end{tabular}

* A fresh enzyme was added after 96 hours. 
nucleosidase, but contain some nuclease, which causes splitting into the mononucleotides, and a nucleotidase, which removes the phosphoric acid. Table 5 illustrates the course of the enzymatic hydrolysis of cozymase.

The isolation of the nicotinamide nucleoside is complicated by the fact that thus far only one reagent has been found which precipitates the compound, namely, phosphotungstic acid. The isolation and purification, therefore, as given in Table 6, consists mainly in removing the other compounds and impurities from the mixture.

The nicotinamide nucleoside gives no characteristic precipitates

Table 6.-Preparation of nicotinamide nucleoside

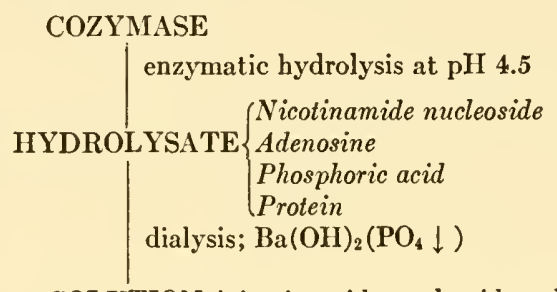

SOLUTION (nicotinamide nucleoside, adenosine)

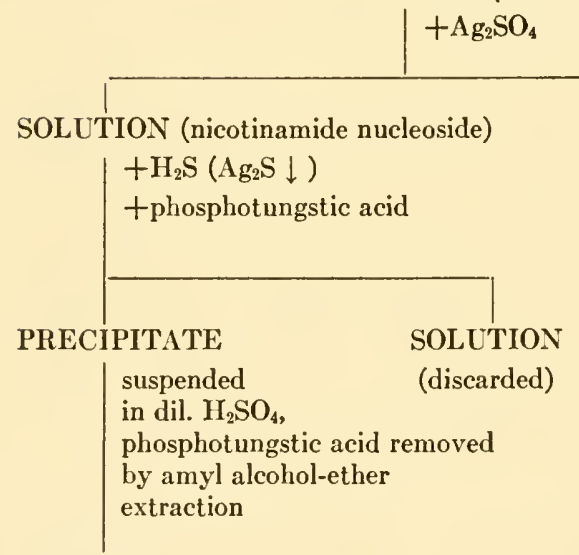

SOLUTION

+ picric acid (impurities $\downarrow$ )

ether extraction;

precipitation by acetone +ether

NICOTINAMIDE NLCLEOSIDE (crude product)

$\mid \begin{aligned} & \text { filtration through } \\ & \text { aluminum oxide; } \\ & \text { fractional precipitation }\end{aligned}$

NICOTINAMIDE NUCLEOSIDE 
with any of the typical reagents used in nucleotide chemistry, such as picric acid, picrolonic acid, Reinecke salt, chloroplatinate, and aurichloride. It is difficult, therefore, to remove the last traces of impurities from the nucleoside preparations. The elementary analysis and the quantitative determination of nicotinamide and pentose, however, rule out every other composition except that of a pentose nucleoside. It remains to be determined what pentose we are dealing with; for this purpose relatively large amounts of the nucleoside must be prepared, a task which is in progress at present.

It was of great interest to compare this split product with the model substances of P. Karrer. It was found to be strikingly similar to the synthetic compounds in all respects. In the first place, the reduction with sodium hydrosulfite should be mentioned (see formula 5).

\section{FORMULA 5}
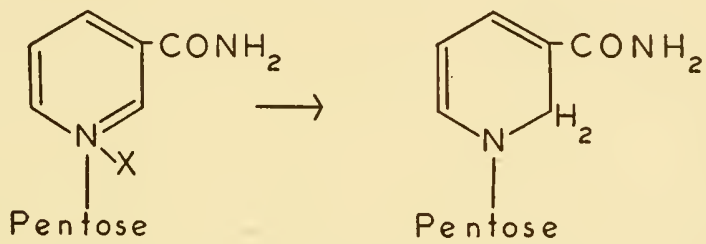

Like the codehydrogenases and the synthetic pyridine derivatives with pentavalent ring nitrogen, the nicotinamide nucleoside yields an $o$-dilhydro compound which has the same characteristic absorption maximum at $340 \mathrm{~m} \mu$ (see Figure 2). In other respects also the nucleoside has the expected properties. It has in the oxidized form

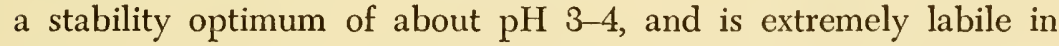
alkali, whereas the dihydro derivative is stable in alkali and is sensitive toward acids, as are the dihydro coenzymes. Alkaline as well as acid hydrolysis separates the carbohydrate from the nicotinamide.

The nucleoside cannot replace either of the coenzymes in the dehydrogenase systems. The phosphoric acid and adenylic acid which are present in the codehydrogenases are necessary for the combination of the pyridinium compound with the apoenzymes.

The investigations of Warburg and Karrer, described above, on the linkage between nicotinamide and the rest of the molecule, which were completed by the isolation of the natural nicotinamide nucleoside, justify the claim of a quaternary pyridinium linkage. Beyond this, our knowledge on the combination of the structural 
units is more complete for cozymase than for codehydrogenase II; investigations on the latter have been less numerous, since it is difficult to obtain in quantity.

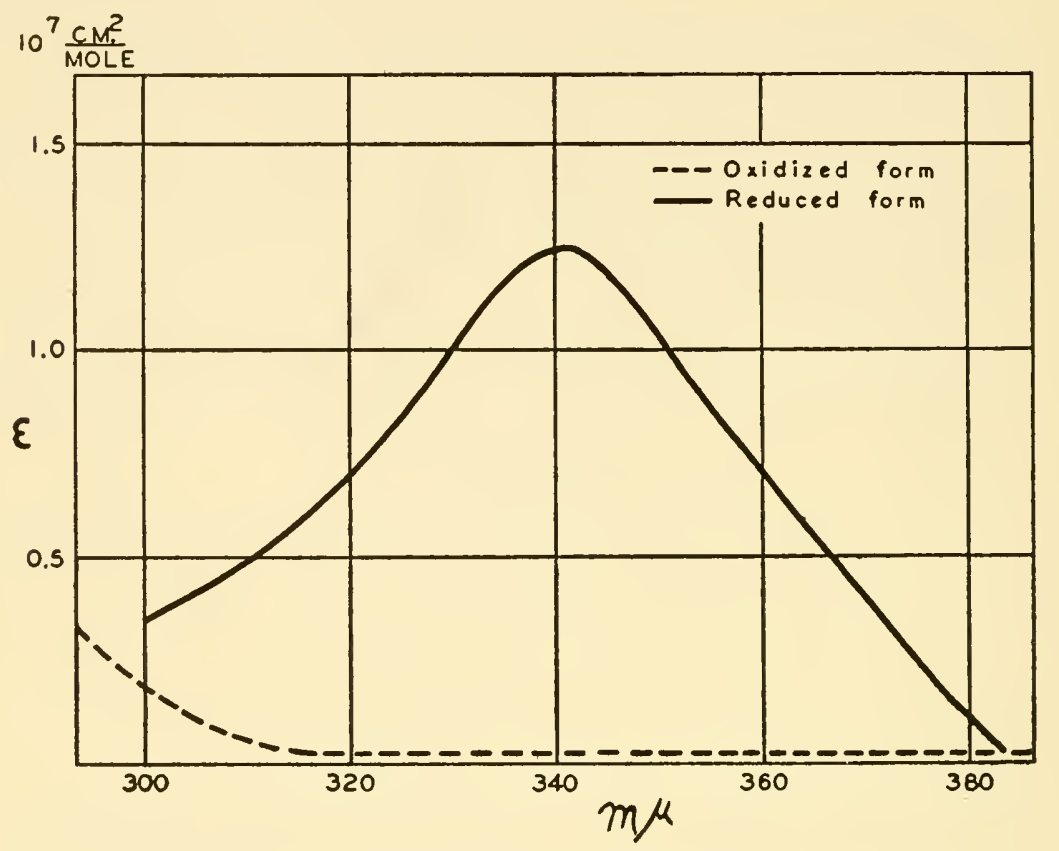

Figure 2.-Absorption spectrum of nicotinamide nucleoside

Our knowledge of the structure of cozymase is based on the following findings: Besides the isolation of nicotinamide and adenine $(6,9)$, the isolation of pentosephosphoric acid was attained by acid hydrolysis (17). By means of the periodate method it was shown that the phosphoric acid is linked to the fifth carbon atom of the pentose molecules, as is indicated in formula 6 .

FORMULA 6<smiles>NC(=O)c1cccnc1</smiles><smiles>Nc1ncnc2[nH]cnc12</smiles><smiles>CC(=O)CC(O)C(O)CC(O)COP(=O)(O)O</smiles> 
Depending on the experimental conditions, the product from alkaline hydrolysis was nicotinamide (28) or adenosine diphosphoric acid (29), the structure of which is well established by the work of Lohmann (30), Embden (31), and Levene (32). Alkaline hydrolysis in the cold gives, besides nicotinamide, a product of adenosine diphosphate plus pentose (formula 7). This degradation product of cozymase is inactive as coenzyme (33).

\section{FORMULA 7}<smiles>NC(=O)c1cccnc1</smiles><smiles>Nc1ncnc2c1ncn2[C@H](O)[C@H](O)[C@H](O)COP(=O)(O)OP(=O)(O)O</smiles><smiles>NC(=O)c1cccnc1</smiles>

The enzymatic hydrolysis gave the two nucleosides:

FORMULA 8
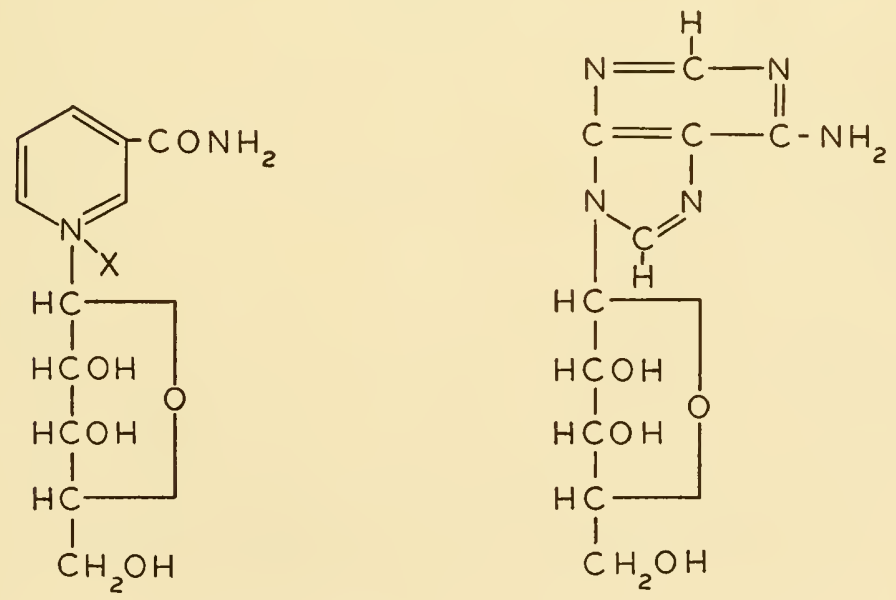
In view of these results and the fact that cozymase is a monobasic acid composed of its structural units minus five molecules of water, the structural formula published in 1936 as a working hypothesis (18) (see formula 9) seems well established. It remains, however, to determine whether or not the pentose of the nicotinamide part is identical with $d$-ribose.

\section{FORMULA 9}
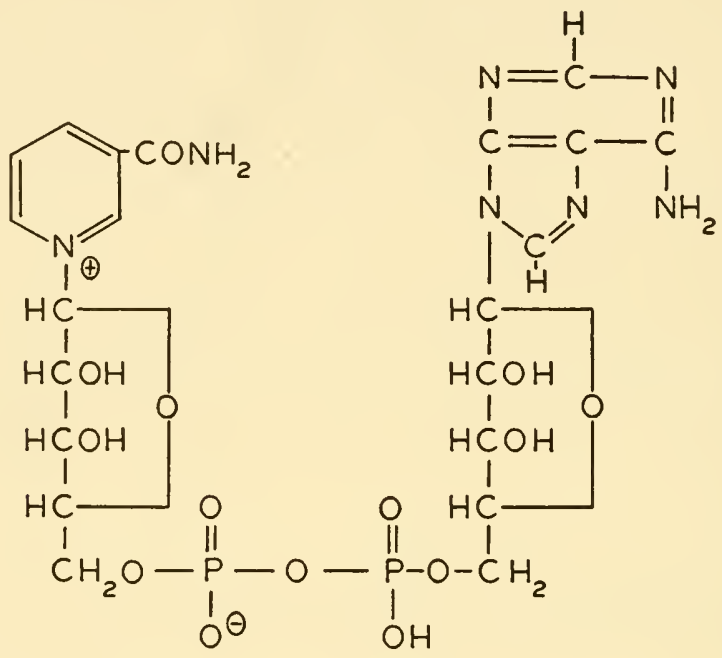

Concerning the structure of codehydrogenase II the following results may be mentioned: Warburg and his co-workers stated that the molecule consists of nicotinamide, adenine, two molecules of pentose, and three molecules of phosphoric acid. These compounds minus six molecules of water form the coenzyme. The experiments on the linkage of the nicotinamide have already been mentioned. In a very early stage of the work, Theorell, on the basis of cataphoretic experiments, claimed that the coenzyme is a tetrabasic acid and that the amino group of the adenine is not free (19). Warburg, Christian, and Griese (5) later revised this claim; their titration experiments indicate that the coenzyme is a tribasic acid, and they showed that the amino group is free. The finding of Adler and Euler (7) that the enzymatic reaction Co $\mathrm{I} \rightleftarrows$ Co II can take place narrowed considerably the possibilities respecting the structure of codehydrogenase II. The location of the third phosphoric acid group now seems to be the only question concerning the structure that has not been settled. The fact that cozymase contains adenosine diphosphate as an essential constituent suggested (15) that codehydrogenase 
II might contain adenosine triphosphate as a part of its molecule (formula 10,1 ).

FORMULA 10

1. Nicotinamide-pentose-

This hypothesis, however, was not substantiated by experimental results. Experiments carried out with rather limited amounts of pure coenzyme II showed that it contains no readily hydrolyzable phosphate, and yields no alkaline degradation product active as a cophosphorylase (34). Therefore it seems probable that the third phosphoric acid group is linked to the adenylic acid part of the molecule, perhaps in much the same way as in yeast adenylic acid (formula $10,2)$. This phosphoric acid group should block the coenzyme properties of the adenylic acid part when liberated by alkaline hydrolysis. Further experimental work is needed to decide this question.

The small amounts of adenosine-5' -phosphoric acid and its homologues which occur as contaminants of impure codehydrogenase preparations and which are formed from alkaline hydrolysis of cozymase but not from codehydrogenase II can be traced by a test

FORMULA 11

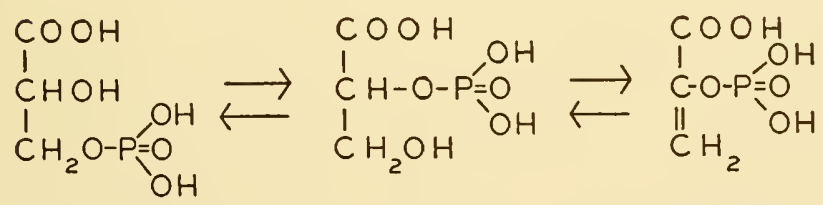

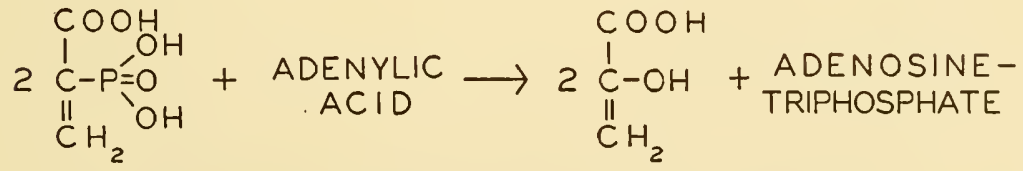

ADENOSINE - TRIPHOSPHATE $\rightarrow$ ADENYLIC ACID 
based on the coenzyme properties of adenylic acid and its homologues (cophosphorylase) in the enzymatic splitting of phosphopyruvic acid (see formula 11). In the absence of a suitable acceptor the phosphoric acid appears in a free state in an amount proportional to the amount of cophosphorylase present in the system; by this method 10 to 100 micrograms of adenylic acid can be determined (35).

\section{Methods of Determination}

Only the methods based on the coenzyme properties will be discussed here. For determining the presence of cozymase Harden (2) originally used a press extract of yeast as a source of apoenzymes and a boiled yeast extract as a source of coenzyme. A great improvement over this method was made when Euler and Myrbäck found that dried brewer's yeast loses its cozymase by repeated extraction with cold water (1e, 36). The remaining product was found to contain all the apoenzymes, activators, and coenzymes necessary for fermentation except cozymase. Therefore it was called apozymase. The use of this preparation is still the simplest and perhaps the most accurate method of determination, its accuracy being limited only by the errors of the manometric measurements. Another test system recently elaborated by Jandorf, Klemperer, and Hastings uses, instead of the function of cozymase in fermentation, the coenzyme properties of cozymase in glycolysis, according to the following scheme (37):

1) Hexose diphosphate $\rightarrow$ phosphoglyceraldehyde + dihydroxyacetone phosphate

2) Phosphoglyceraldehyde $+\mathrm{H}_{3} \mathrm{AsO}_{4} \rightarrow$ arsenophosphoglyceraldehyde

3) Arsenophosphoglyceraldehyde + DPN $\rightarrow$ arsenophosphoglyceric acid + DPN- $\mathrm{H}_{2}$

4) Arsenophosphoglyceric acid $\rightarrow$ 3-phosphoglyceric acid + $\mathrm{H}_{3} \mathrm{AsO}_{4}$

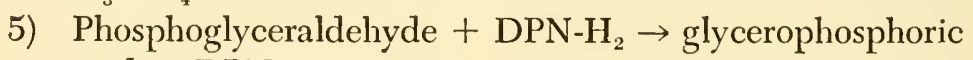
acid + DPN

6) Hexose diphosphate $\rightarrow$ 3-phosphoglyceric acid + glycerophosphoric acid

The amount of phosphoglyceric acid produced in a given time in the presence of bicarbonate buffer can be measured manometrically with the Warburg apparatus. The test system for codehydrogenase II as given by Warburg uses the dehydrogenation of Robison ester 
by codehydrogenase II, Robison ester apodehydrogenase (Zwischenferment), and ribollavin enzyme. If the codehydrogenase II is the speed-limiting factor in this system, the oxygen uptake is a measure of its concentration (5). It is probable that the old yellow enzyme used by Warburg and Christian is an artifact. The cytochrome $c$ reductase of Haas, Horecker, and Hogness (38) seems to be the natural acceptor for the hydrogen of the reduced codehydrogenase II.

These methods of determination have been used not only to elaborate the purification of the codehydrogenases but also to study their distribution from the standpoint of vitamin research. The codehydrogenases occur in an equilibrium: coenzyme $\rightleftarrows$ dihydrocoenzyme. Since the stabilities of the oxidized and the reduced forms are different (see Table 4), the proportions of coenzyme and dihydrocoenzyme can be determined by heat extraction with acid or with alkali, according to Adler and Calvett (39). If the extraction is made with boiling water, the reduced coenzyme is oxidized by air. Therefore no loss occurs if some of the subsequent steps of the preparation are carried out in acid medium.

In most tissues somewhat more of the oxidized than of the reduced form of cozymase was found, but in the Jensen rat sarcoma, Euler and his co-workers found a large excess of dihydrocozymase $(20,40)$. Recent experiments confirm this finding, but apparently it is not true for all cancerous tissues. An excess of dihydrocozymase was repeatedly found in methyl-cholanthrene rat tumor, but not regularly in benzpyrene tumors of mice and in Brown-Pearce rabbit carcinoma (33). The data on human carcinomata are still too limited to permit any conclusion.

It must be remembered that all methods of determination in tissues involve complications: incompleteness of extraction, limited stability of the codehydrogenases in oxidized and reduced form at high temperature, rapid changes in the equilibrium Co I $\rightleftarrows$ Co II, enzymatic destruction upon disruption of the cells, and finally errors in the methods of determination. The claims for accuracy which are made by many publications dealing with coenzyme content of tissues seem far too optimistic.

The growth-promoting properties of the codehydrogenases for certain microorganisms (Hemophilus infucnzae and Hemophilus para-influenzae) can be used for determining small quantities of these compounds (41). Whereas the number of nicotinamiderequiring microorganisms is rather high, only $H$. influenzae and 
H. para-influenzae have been found to require cozymase or codehydrogenase II. According to Lwoff only 0.004 microgram per milliliter of peptone solution is necessary to produce visible growth under standard conditions. The method does not distinguish between codehydrogenases I and II. Dihydrocozymase was found to be inferior as a nutrilite to an equivalent amount of cozymase (42). This may be due to a difference in the permeability of the cells to the two compounds.

For the examination of pure or almost pure coenzyme preparations the spectrophotometric determination of the dihydro compounds is a very accurate method. The solutions must, however, be free from impurities that absorb in the ultraviolet region in which the dihydrocoenzymes exhibit their characteristic absorption. The chemical methods of determination cannot be discussed here in detail.

\section{The Apodehydrogenases}

Since the history of the apodehydrogenases has been reviewed in several comprehensive articles (la, c, g, 43a-d), they will simply be listed here, and our present knowledge about their purification, nature, and function will be summarized and their coenzyme specificity discussed.

As can be seen from Table 7, some of the apodehydrogenases have been prepared in a pure state and obtained in a crystalline form. The main progress in the field was made by Warburg and his coworkers, especially Christian, Negelein, Gerischer, Haas, Wulff, and Kubowitz. Warburg's methods for purification consist mainly in precipitations of the apoenzymes at the isoelectric point, their fractional precipitation by ammonium sulfate or organic solvents, and removal and inactivation of other enzymes by heat denaturation at a temperature of about $50^{\circ} \mathrm{C}$. Of special interest is the precipitation of the diphosphoglyceraldehyde apodehydrogenase in a step of its purification by addition of nucleic acid (44).

The isolation of the apodehydrogenases in a pure state was important for a detailed study of their relation to coenzymes and substrate by the spectrophotometric technique. Whereas the use of crude apoenzymes does not exclude the possibility of more complex reactions, a definite conclusion can be drawn respecting the mechanism of a reaction if pure apoenzyme, coenzyme, and substrate are used. Our previous conception of the function of cozymase in the dehydrogenation of triosephosphate was greatly revised in 


\section{Table 7.-The apodehydrogenases dependent on nicotinamide nucleotides}

\begin{tabular}{ccccc}
\hline \hline $\begin{array}{c}\text { Substrate and dehydrogenation } \\
\text { product }\end{array}$ & $\begin{array}{c}\text { Co- } \\
\text { enzyme }\end{array}$ & Note on the apodehydrogenase & Ref. \\
\hline $\begin{array}{c}\text { 1,3-diphosphoglyceraldehyde } \rightleftarrows \\
\text { 1,3-diphosphoglyceric acid }\end{array}$ & Co I & $\begin{array}{c}\text { Obtained by Warburg and Christian } \\
\text { in crystalline state from yeast }\end{array}$ \\
$\begin{array}{c}\text { Triosephosphate } \rightleftarrows \text { phospho- } \\
\text { glyceric acid }\end{array}$ & Co I & $\begin{array}{c}\text { Crude preparations from animal tis- } \\
\text { sues. Identical with 1,3-diphospho- 71 } \\
\text { glyceraldehyde apodehydrogenase? }\end{array}$
\end{tabular}

Lactic acid $\rightleftarrows$ pyruvic acid

Alcohol $\rightleftarrows$ acetaldehyde

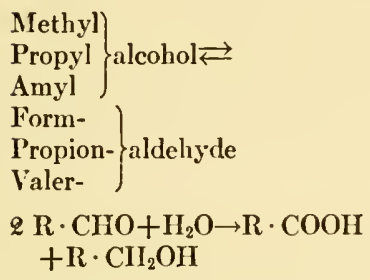

Malic acid $\rightleftarrows$ oxalacetic acid

$\alpha$-glycerophosphate $\rightleftarrows$ phosphoglyceraldehyde

$\beta$-hydroxybutyric acid $\rightleftarrows$ acetoacetic acid

Formic acid $\rightleftarrows \mathrm{CO}_{2}$

Glucose-6-monophosphate (Robison ester) $\rightarrow$ phosphohexonic acid

Phosphohexonic acid

Decarboxylation and dehydrogenation

Isocitric acid $\rightleftarrows \alpha$-keto- $\beta$-carboxyglutaric acid

Glucose $\rightleftarrows$ gluconic acid

Glutamic acid

Iminoglutaric acid
Co I Prepared by Straub in a crystalline 72 form from heart

Co I Obtained by Negelein and Wulff in 51 crystalline form from yeast

Co I Crude preparations from animal tissues. Identical with ethyl alcohol apodehydrogenase?

Co I Aldehyde mutase; crude prepara- 73 tions from liver

Co I Crude preparations from animal tis- 74 sues

Co I Crude preparations from yeast and 75 tissues

Co I Crude preparations from animal tis- $\quad 76$ sues

Co I Crude preparations from seeds and 49 B. coli

Co II Obtained from yeast in a lighly 77 purified state by Negelein and Gerisclier

Co II Purified preparation obtained from 78 yeast by Warburg and Christian

Co II Crude preparations from seeds, ani- 79 mal tissues, and yeast

Co I or Crude preparations from liver and 45 Co II yeast

Co II For apoenzyme from yeast and $B .80$ coli;

Co I or For apoenzyme from animal tissues;

Co II

Co I For apoenzyme from plants 
this way by Warburg and Christian. They showed that diphosphoglyceraldehyde rather than triosephosphate is the substrate of cozymase in fermentation and perhaps in glycolysis (44). The main purpose of giving, in Table 7 , the degree of purity of the apoenzymes obtained thus far is to afford some idea of the reliability of our knowledge respecting the reactions in question.

The most noteworthy fact regarding the apoenzymes is that they are responsible for the specificity of the dehydrogenase, whereas codehydrogenases I and II combined with different proteins form a relatively large number of dehydrogenases. The protein moiety is therefore more specific than the prosthetic group.

It was found that both codehydrogenase I and codehydrogenase II can act as coenzymes for glucose apodehydrogenase (45). This apoenzyme, however, has not yet been sufficiently purified, and therefore it is not impossible that a transformation of codehydrogenase I into codehydrogenase II or vice versa may occur in this system.

In connection with alcohol apodehydrogenase it should be mentioned that methyl, propyl, and amyl alcohol can also serve as substrate (46). This seems to indicate that the specificity of the apodehydrogenases even toward the substrate is not always an absolute one.

Warburg has claimed that the protein part of a given dehydrogenase probably differs for each type of cells. Robison ester apodehydrogenase from yeast and from rat blood were found to be different, their isoelectric points being at $\mathrm{pH} 4.8$ and 5.8 respectively (1b). In some cases the difference is so great as to suggest that the specificity for the codehydrogenases depends on the source of the apoenzyme. Thus glutamic acid apodehydrogenase from liver was found by Adler to require codehydrogenase II; the apoenzyme from plants uses codehydrogenase I as a prosthetic group (47). Besides the cozymase-dependent glycerophosphate apodehydrogenase, a glycerophosphate dehydrogenase was found to occur in muscle tissue which does not require a coenzyme (48). Similar findings have been reported for formic dehydrogenase. Whereas the dehydrogenase preparations from seeds consist of cozymase plus apoenzyme (49), the corresponding enzyme from Bacterium coli does not need cozymase (50).

If there are actually as many variations of apodehydrogenases as there are different types of cells, a further important specificity may be based on this difference. To obtain more evidence, the apode- 
hydrogenases must be prepared from different sources and the resulting products compared with one another. It is probable that the differences will consist in a slight variation in the $\mathrm{pH}$ for optimum activity or a change in the equilibrium between protein, substrate, and coenzyme rather than in a completely different mode of action.

The preparation of some apodehydrogenases in crystalline form has permitted an estimation of the amount present in the cell. They are found in much smaller concentration than the codehydrogenases. O. Meyerhof (1c) has pointed out that if each cozymase molecule in rabbit muscle or yeast were accompanied by one molecule of each of the apodehydrogenases, the protein of the cells would consist exclusively of the apodehydrogenases.

The apodehydrogenases combine with the nicotinamide nucleotides, the reduced nucleotides, the substrate, and the reaction product. The extent to which the complexes thus formed are dissociated determines the direction and the rate and equilibrium of the reaction. As an example may be mentioned the following system: alcohol, acetaldehyde, cozymase, dihydrocozymase, and apodehydrogenase. This system was investigated by Negelein and Wulff, who used the pure components (51). They found that under their experimental conditions the concentration of each substance at which the apodehydrogenase is half saturated with it is as follows: cozymase, 0.0001 M.; dihydrocozymase, 0.00003 M.; acetaldehyde, $0.0001 \mathrm{M}$; and ethyl alcohol, $0.024 \mathrm{M}$. The reduced coenzyme and acetaldehyde are bound to a much greater extent than cozymase and alcohol, and under normal conditions the reversible reaction acetaldehyde + dihydrocozymase $\rightleftarrows$ alcohol + cozymase proceeds in the direction of alcohol formation. Experiments carried out on other pyridine dehydrogenase systems have yielded similar results. The dehydrogenases requiring codehydrogenase II, however, seem to be less dissociated.

The high degree of dissociation between the pyridine nucleotides and their protein parts corresponds to that of the cophosphorylases and their specific proteins. M. Dixon and L. G. Zerfas have pointed out the differences between this group of enzymes and the other type, in which the prosthetic group is in a relatively stable linkage to the protein and the ratio between prosthetic group and protein is $1: 1(52)$. On the basis of interesting experimental results with artificial hydrogen acceptors they state in their discussion that the use of the term "pyridine-proteid" is misleading and should be discontinued. It would seem, however, that this suggestion is too radi- 
cal. The work that has been done in this field leaves no doubt that we are dealing with real enzymes, which, after bringing about the dehydrogenation of substrate under biological conditions, are unchanged. Furthermore, at the moment when they exhibit their activity, the nicotinamide nucleotide is combined with its specific protein just as the ribollavin nucleotide is combined with its specific protein. That we deal in the one case with a relatively stable linkage and in the other with a very unstable one is only a difference in degree. Hence a change of the whole nomenclature would seem to be unnecessary and would probably increase the confusion in this field.

The physiological reoxidation of the reduced coenzymes is brought about by the oxidized form of the metabolites listed in Table 7, as, for example, acetaldehyde in fermentation or pyruvic acid in glycolysis. In other instances the dihydrocoenzymes are reoxidized by alloxazine proteids such as diaphorase (84), coenzyme factor (85), cytochrome $c$ reductase (38), or the enzyme recently detected by Altschul, Persky, and Hogness (86).

\section{Spectrophometric Methods}

In studying the function of the nicotinamide nucleotide enzymes we have been greatly aided by the spectrophometric methods developed by Warburg. The basis is the appearance of an absorption band at $340 \mathrm{~m} \mu$ when the codehydrogenases are reduced. This absorption band disappears upon reoxidation. Under proper experimental conditions the concentration of coenzyme or apoenzyme, the substrate and acceptor specificity of the dehydrogenases, and the speed of reactions can be studied, as has been done in some laboratories, notably those of Warburg, Euler, and Meyerhof (1b, i; 53).

As an example the action of the reducing and the oxidizing fermentation system may be given (44). See Figure 3.

\section{Biosynthesis of the Codehydrogenases}

In view of the relatively complicated configuration of the codehydrogenases and of their nucleotide character, it is improbable that a satisfactory synthesis will be obtained by present-day methods of organic chemistry. Therefore experiments designed to carry out a biosynthesis from the structural units are very important from a practical point of view.

Mention may be made first of the experiments dealing with the 
formation of the codehydrogenases from nicotinamide in the organism. The biological function of the nicotinamide-containing coenzymes and their wide distribution in nature (1f) were known before their vitamin properties. There was little doubt that the nicotinamide ingested as a vitamin is used to form the coenzymes. Nevertheless an exact demonstration had to be obtained.

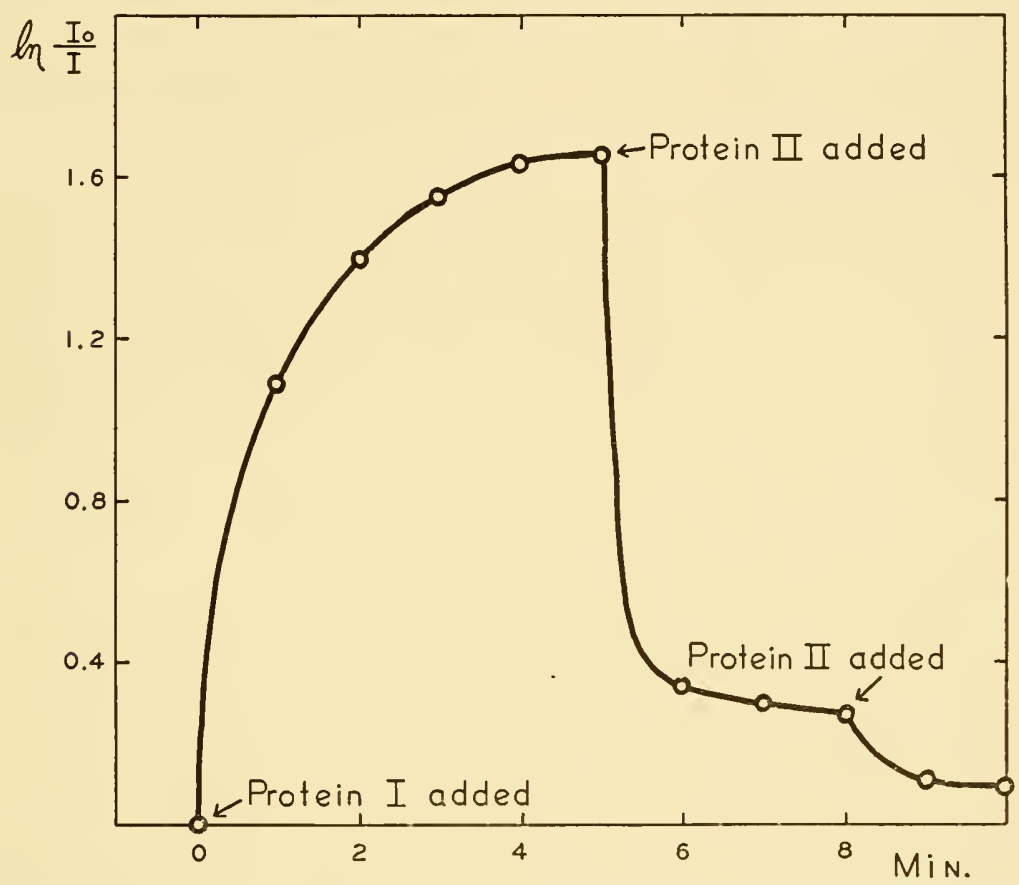

Figure 3.-Action of oxidizing and reducing fermentation enzymes. Spectrophotometric experiment, absorption at $340 \mathrm{~m} \mu$ (dihydrocozymase). $\mathrm{d}=0.557$ cm. Protein I: diphosphoglyceraldehyde apodehydrogenase. Protein II: acetaldehyde reductase (alcohol apodehydrogenase). The concentrations are as follows: cozymase, $0.183 \mathrm{mg}$. per $\mathrm{ml}$; $d$-phosphoglyceraldehyde, $0.733 \mathrm{mg}$. per $\mathrm{ml}$; acetaldehyde, $1.47 \mathrm{mg}$. per ml.; apoenzymes (protein I and II), $0.0008 \mathrm{mg}$. per ml.; orthophosphate, $3.3 \times 10^{-5}$ mole per ml.; pyrophosphate, $3.3 \times 10^{-3}$ mole per $\mathrm{ml}$.

Experiments on animal tissues were performed by Axelrod and Elvehjem. The cozymase level of pigs and dogs living on a diet low in nicotinamide was found to be decreased in muscle and liver. Upon administration of nicotinamide the normal cozymase content was rapidly restored (56). Later the same effect was observed in pellagrins by Axelrod, Spies, and Elvehjem (58). Experiments on rats also seemed to indicate the same result (57), but according 
to more recent findings the vitamin nature of nicotinamide for the rat is doubtful $(81,82)$. Dann and Handler recently have shown that nicotinic acid is formed by the chick embryo (83).

An important observation made in these experiments was that the organism does not synthesize coenzyme beyond the normal level of coenzyme content under favorable dietary conditions. Even an administration of nicotinamide far in excess of the normal requirement results in no significant synthesis beyond the normal level. In erythrocytes, however, an increase in coenzyme content upon administration of an excess of nicotinamide was observed by Kohn and Klein, Vilter and Spies, Axelrod and others $(59,60)$.

Numerous experiments with isolated enzyme preparations from yeast, liver, and muscle have thus far failed to give a noteworthy synthesis of the coenzymes from the structural units. The destructive tendency of these preparations has always been found to be remarkable. It was observed by Euler and co-workers (64-66) that in tissues the coenzyme content after death, and especially upon destruction of the cell structure, decreases rapidly. The significance of this finding for the methods of determination and the precautions necessary have been pointed out repeatedly.

Mann and Quastel have recently confirmed the findings of Euler. Of special interest is their finding that free nicotinamide in great excess prevents the postmortem decomposition of cozymase (67), a result which the authors explain by assuming that nicotinamide and cozymase compete for the active center of the nucleotidase which destroys the coenzyme.

Lennerstrand found some years ago $(68,69)$ that a destruction of cozymase by washed dried yeast (apozymase) takes place, but is inhibited by phosphate and hexosediphosphate. Apparently the substrate protects the coenzyme from destruction. It is not yet possible to say what products are formed by the inactivation of cozymase by apozymase. If glucose and phosphate are added to the inactivated cozymase plus apozymase, a resynthesis takes place after several hours, and as much as 50 per cent of the cozymase originally present is restored.

A similar effect was observed recently with cocarboxylase. When cocarboxylase is incubated with aetiozymase, it is destroyed. In the presence of pyruvate a much slower inactivation takes place (33). It is possible that underlying these findings is an important principle of regulation of the coenzyme level.

It seemed possible that a regeneration of cozymase from the nico- 
tinamide nucleoside might take place in a fermentation system, similar to the formation of cocarboxylase from thiamine. Experiments designed to carry out such a biosynthesis of cozymase by incubation with yeast preparations in the presence of phosphate and adenosine phosphoric acids have not yet been successful. Nevertheless it can be assumed that the nicotinamide nucleoside is an intermediate in the course of biosynthesis of the codehydrogenases.

Among the lower organisms of the plant kingdom-for example, Bacterium aerogenes, Torula, and other yeasts-we have examples of biosynthesis of cozymase from very simple nitrogen and carbon sources. Hutchens, Jandorf, and Hastings have shown recently that the protozoon Chilomonas paramecium is capable of synthesizing cozymase in a synthetic medium containing ammonia as the only source of nitrogen and acetate as the sole source of carbon (62). Other microorganisms, such as Staphylococcus aureus (54), Proteus vulgaris (55), and Shigella paradysenteriae (63), require nicotinamide to complete the synthesis of cozymase, a fact which permits these organisms to be used for the bio-assay of nicotinamide. It has been suggested that these bacteria require nicotinamide for growth as a result of their parasitic existence (55). Most striking in this connection is the finding of Lwoff and Lwoff that Hemophilus influenzae and para-influenzae require codehydrogenase I and II (41). This specificity surpasses even that of man and other mammals.

Gingrich (42) has shown that the " $V$ " requirement of hemophilic bacteria can be satisfied not only by the oxidized codehydrogenases but also by dihydrocozymase, acid-treated dihydrocozymase (completely inactive as codehydrogenase), and desamino cozymase, an artificial derivative of cozymase (61) in which the adenylic acid is replaced by inosinic acid. Furthermore, the fact that the nicotinamide nucleoside has been found (42) to promote the growth of these organisms is highly interesting in that it demonstrates that the only special requirement is the preformed linkage of nicotinamide to the pentose. It is obvious, then, that the nutrilites required for the biosynthesis of cozymase are related chiefly to the nicotinamide moiety and its linkage to the rest of the molecule. The steps in biosynthesis between simple nitrogen and carbon compounds and the pyridine ring remain a promising field of investigation.

\section{REFERENCES}

1. a) Thunberg, T., Ergebnisse d. Enzymforschung (Nord-Weidenhagen), 7, 163 (1938). 
b) Warburg, O., Ergebnisse d. Enzymforschung, 7, 210 (1938).

c) Meyerhof, O., Ergebnisse d. Physiologie (Asher-Spiro), 39, 10 ( 1937 ).

d) v. Euler, H., Ergebnisse d. Physiologie, 38, 1 (1936).

e) Мүнвӓск, K., Ergebnisse d. Enzymforschung, 2, 139 (1933).

f) Мүввӓск, K., Tabulae Biologicae, 14, 110 (1937).

g) Ball, E. G., Bull. Johns Hopkins Hosp., 65, 253 ( 1939).

h) Schlenk, F., and v. Euler, H., Fortschritte d. Chemie org. Naturstoffe (Zechmeister), 1, 99 (1938).

i) Schlenk, F., and Günther, G., Methodik d. Fermente (BamannMyrbäck) part 7 (1940).

k) Baumann, C. A., and Stare, F. J., Physiol. Rev., 19, 353 (1939).

2. Harden, A., and Young, W. J., Proc. Roy. Soc. (London) B 77, 405 (1906).

Harden, A., Alcoholic Fermentation, 4th ed. (London, 1932).

3. Warburg, O., and Christian, W., Biochem. Z., 242, 206 (1931); 254, 438 ( 1932 ).

4. Warburg, O., and Christian, W., Biochem. Z., 274, 112 (1934); 275, 464 ( 1935$)$.

5. Warburg, O., Christian, W., and Gruese, W., Biochem. Z., 282, 157 (1935).

6. v. Euler, H., Albers, H., and Schlenk, F., Z. physiol. Chem., 237, I (1935).

7. Adler, E., Elliot, S., and Elliot, L., Enzymologia, 8, 80 ( 1940 ).

8. Warburg, O., and Christian, W., Biochem. Z., 287, 291 (1936).

9. v. Euler, H., Albers, H., and Schlenk, F., Z. physiol. Chem., 240, 113 (1936).

10. Ohlmeyer, P., Biochem. Z., 297, 66 (1938).

11. OchoA, S., Biochem. Z., 292, 68 (1937).

12. Williamson, S., and Green, D. E., J. Biol. Chem., 135, 345 (1940).

13. JandorF, B. J., J. Biol. Chem., 138, 305 (1941).

14. Schlenk, F., Svenska Vet. Akad. Arkiv f. Kemi, 12 A, 21 ( 1937 ).

15. v. Euler, H., and Schlenk, F., Z. physiol. Chem., 246, 64 ( 1937 ).

16. v. Euler, H., and Schlenk, F., Svensk Kem. Tidskr., 48, 135 ( 1936 ).

17. Schlenk, F., Svenska Vet. Akad. Arkiv f. Kemi, 12 B, 20 ( 1936).

18. Schlenk, F., and v. Euler, H., Naturwissenschaften, 24, 794 (1936).

19. Theoreli, H., Biochem. Z., 275, 19 ( 1934).

20. v. Euler, H., Schlenk, F., Herwinkel, H., and Högberg, B., Z. physiol. Chem., 256, 208 (1938).

21. Adler, E., Hellström, H., and v. Euler, H., Z. physiol. Chem., 242, 225 (1936).

22. v. Euler, H., Adler, E., and Hellström, H., Z. physiol. Chem., 241, $239(1936)$.

23. a) Karrer, P., Schwanzenbach, G., Benz, F., and Solmssen, U., Helv. Chim. Acta, 19, 811, 1028 (1936).

b) Karrer, P., Ringier, B. H., Büchi, J., Fritzsche, H., and Solmissen, U., Helv. Chim. Acta, 20, 55 (1937).

c) Kamrer, P., Scinwarzenbach, G., and Utzinger, G. E., Helv. Chim. Acta, 20, 720 ( 1937).

d) Karrer, P., and Stare, F. J., Helv. Chim. Acta, 20, 418 (1937).

e) Karrer, P., Kahnt, F. W., Epstein, R., Jaffé, W., and Ishi, T., Helv. Chin. Acta, 21, 223 ( 1938).

f) Karrer, P., Ishit, T., Kaitnt, F. W., and van Bergen, I., Helv. Chim. Acta, 21, 1174 (1938).

24. Mүrв̈̈ск, K., and Öntenblad, B., Z. physiol. Chem., 233, 87 (1935). 
Karrer, P., Schlenk, F., and v. Euler, H., Svenska Vet. Akad. Arkiv f. Kemi, 12 B, 26 (1936).

25. HaAs, E., Biochem. Z., 285, 368 (1936).

26. SchleNk, F., Naturwissenschaften, 28, 46 (1940).

27. Bredereck, H., Beuchelt, H., and Richter, G., Z. physiol. Chem., 244, 102 (1936); Ber. Chem. Ges., 71, 408 (1938).

28. Schlenk, F., v. Euler, H., Heiwinkel, H., Gleim, W., and Nyström, H., Z. physiol. Chem., 247, 23 (1937).

29. Vestin, R., Schlenk, F., and v. Euler, H., Ber. Chem. Ges., 70, 1369 (1937).

30. Lohmann, K., Biochem. Z., 282, 120 (1935).

31. Embden, G., and Zimmermann, M., Z. physiol. Chem., 167, 114, 137 (1927).

32. Levene, P. A., and Bass, L. W., Nucleic Acids (New York, 1931), pp. 187-192.

33. SCHLENK, F., unpublished experiments.

34. Schlenk, F., Högberg, B., and Tingstam, S., Svenska Vet. Akad. Arkiv f. Kemi, 13 A, 11 (1939).

35. Schlenk, F., and Schlenk, T., J. Biol. Chem., 141, 311 (1941).

36. Мүнвӓск, K., Z. physiol. Chem. 177, 158 (1928).

Schlenk, F., and Vowles, R. B., Svenska Vet. Akad. Arkiv f. Kemi, $13 B, 19$ (1940).

37. Jandorf, B. J., Klemperer, F. W., and Hastings, A. B., J. Biol. Chem., 138, 311 (1941).

38. HaAs, E., Horecker, B. L., and Hogness, T. R., J. Biol. Chem., 136, 747 (1940).

39. Adler, E., and Calvetr, F., Svenska Vet. Akad. Arkiv f. Kemi, 12 B, 32 (1936).

40. v. Euler, H., Malmberg, M., and Günther, G., Z. f. Krebsforschung, 45, 425 (1937).

41. Lwoff, A., and Lwoff, M., Comptes rendus Acad. Sci., 203, 896 (1936); Proc. Roy. Soc. (London) B 122, 352, 360 (1937).

42. Gingrich, W. D., and Schlenk, F., unpublished experiments.

43. a) Oppenheimer, C., and Stern, K. G., Biological Oxidation (The Hague, 1939).

b) Thunberg, T., Ergebnisse d. Physiologie, 39, 76 (1937).

c) Franke, W., in Euler, Chemie d. Enzyme, 2, 3 (1934).

d) Potter, V. R., Medicine, 19, 441 (1940).

44. Warburg, O., and Christian, W., Biochem. Z., 303, 40 (1939).

45. Das, N. B., Z. physiol. Chem., 238, 269 (1936).

Quibell, T. H., Z. physiol. Chem., 251, 102 (1938).

46. Lutwak-ManN, C., Biochem. J., 32, 1364 (1938).

47. v. Euler, H., Adler, E., Günther, G., and Das, N. B., Z. physiol. Chem., 254, 61 (1938).

48. Green, D. E., Biochem. J., 30, 629 (1936).

49. Adler, E., and Sreenivasaya, M., Z. physiol. Chem., 249, 24 (1937).

50. Gale, E. F., Biochem. J., 33, 1012 (1939).

51. Negelein, E., and WulfF, H. J., Biochem. Z., 293, 351 (1937).

52. Dixon, M., and Zerfas, L. G., Biochem. J., 34, 371 (1940).

53. Meyerhof, O., Ohlmeyer, P., and Möhle, W., Biochem. Z. 297, 90 (1938).

54. Knight, B. C. J. G., Biochem. J., 31, 731, 966 (1937).

55. Fildes, P., Proc. Roy. Soc. (London), B 124, 4 (1937); Brit. J. of Exp. Pathol., 19, 239 (1938). 
56. Axelrod, A. E., Madden, R. J., and Elvehjem, C. A., J. Biol. Chem., 131,85 (1939).

DanN, W. J., and Handler, P., J. Nutrition, 22, 409 (1941).

57. v. Euler, H., Schlenk, F., Melzer, L., and Högberg, B., Z. physiol. Chem., 258, 212 (1939).

Frost, D. V., and Elvehjear, C. A., J. Biol. Chem., 121, 255 (1937).

58. Axelrod, A. E., Spies, T. D., and Elvehjem, C. A., J. Biol. Chem., 138, 667 (1941).

59. Kohn, H. I., and KLeIN, J. R., J. Biol. Chem., 130, 1 (1939); 135, 685 (1940).

60. Vilter, R. W., Vilter, S. P., and Spies, T. D., J. Am. Med. Assoc., 112, 420 (1939); Vilter, S. P., Koch, M. B., and Spies, T. D., J. Lab. Clin. Med., 26, 31 (1940).

Axelrod, A. E., Gordon, E. S., and Elvehjem, C. A., Am. J. Med. Sci., 199,697 (1940).

61. Schlenk, F., Hellström, H., and v. Euler, H., Ber. Chem. Ges., 71, 1471 (1938).

62. Hutchens, J. O., Jandorf, B. J., and Hastings, A. B., J. Biol. Chem., 138, 321 ( 1941$)$.

63. a) Dorfman, A., Koser, S. A., Reames, S. A., Swingle, R. H., and SaunDERs, F., J. Inf. Diseases, 65, 163 (1939).

b) Dorfman, A., Koser, S. A., and Saunders, F., Proc. Soc. Exp. Biol. Med., 43, 434 (1940).

c) Saunders, F., Dorfman, A., and Koser, S. A., J. Biol. Chem., 138, 69 (1941).

d) Bass, A., Berkman, S., Saunders, F., and Koser, S. A., J. Inf. Diseases, 68,175 (1941).

64. v. Euler, H., Myrbäck, K., and Brunius, E., Z. physiol. Chem., 177, 237; 183, 60 (1929).

65. v. Euler, H., and Günther, G., Z. physiol. Chem., 243, 1 (1936).

66. v. Euler, H., Heiwinkel, H., and Schlenk, F., Z. physiol. Chem., 247, IV (1937).

67. Mann, P. J. G., and Quastel, J. H., Biochem. J., 35, 502 (1941).

68. LenNerstrand, Å., Biochem. Z., 287, 172 (1936).

69. Lennerstrand, A., Svenska Vet. Akad. Arkiv f. Kemi, 14 B, 1 (1940); 14 A, 16 (1941).

70. Adler, E., and Günther, G., Z. physiol. Chem., 253, 143 (1938).

71. Meyerhof, O., Kiessling, W., and Schulz, V., Biochem. Z., 292, 25 (1937).

72. Straub, F. B., Biochem. J., 34, 483 (1940).

73. Dixon, M., and Lutwak-ManN, C., Biochem. J., 31, 1347 (1937). Adler, E., v. Euler, H., and GüNther, G., Svenska Vet. Akad. Arkiv f. Kemi, 12 B, 54 (1938).

74. v. Euler, H., Adler, E., and Günther, G., Z. physiol. Chem., 249, 1 (1937).

Green, D. E., Biochem. J., 30, 2095 (1936).

75. Anler, E., v. Euler, H., and Hughes, W., Z. physiol. Chem., 252, 1 (1938).

76. Green, D. E., Dewan, I. G., and Lelorr, L. F., Biochem. J., 31, 934 (1937).

77. Negelein, E., and Gerischer, W., Biochem. Z., 284, 289 (1936).

78. Warburg, O., and Christian, W., Biochem. Z., 292, 287 (1937).

79. Adler, E., v. Euler, H., Günther, G., and Plass, M., Biochem. J., 33, 1028 (1939). 
80. v. Euler, H., Adler, E., Günther, G., and Elliot, L., Enzymologia, 6, 337 (1939).

v. EULER, H., and Adler, E., Enzymologia, 7, 21 (1939).

GüNTHER, G., Svenska Vet. Akad. Arkiv f. Kemi, 12 A, 23 (1938).

81. Dann, W. J., and Kohn, H. I., J. Biol. Chem., 136, 435 (1940).

DanN, W. J., J. Biol. Chem., 141, 803 (1941).

82. Elvehjem, C. A., Biological Action of the Vitamins. Lecture at the Chicago sessions of the Symposium on Enzymes and Vitamins, 1941.

83. Dann, W. J., and Handler, P., J. Biol. Chem., 140, 935 (1941).

84. Adler, E., v. Euler, H., Günther, G., and Plass, M., Skand. Arch. Physiol., 82, 6 (1939).

LOCKHART, E. E., Biochem. J., 33, 613 (1939).

Abraham, E. P., and Adler, E., Biochem. J., 34, 119 (1940).

85. Corran, H. S., Green, D. E., and Straub, F. B., Biochem. J., 33, 793 (1939).

86. Altschul, A. M., Perskx, H., and Hogness, T. R., Science, 94, 349 (1941). 


\title{
The Flavoproteins
}

\author{
T. R. HOGNESS \\ University of Chicago
}

7 THE flavoproteins constitute a relatively large class of the 1 respiratory enzymes. As we know them today they are characterized by having as prosthetic groups either alloxazine mononucleotide, i.e., riboflavin phosphate, or alloxazine adenine dinucleotide, which is composed of both riboflavin phosphate and adenylic acid. They are further characterized by their reactivity toward oxygen-some to a very limited extent-when in the reduced form. Many of them are oxidized by methylene blue; one is oxidized by fumaric acid; and one is specifically oxidized by a known member of the hydrogen transport system: the flavoprotein cytochrome $c$ reductase, in its reduced state, is oxidized by cytochrome $c$.

In the oxidized state the flavoproteins as a group can be reduced by a variety of substrates-dihydrotriphosphopyridine nucleotide (cozymase II), dihydrodiphosphopyridine nucleotide (cozymase I), the $d$-amino acids, xanthine and other purines, and some of the aldehydes. Each particular flavoprotein, however, is specific toward one (or one class) of the above substrates. The chemical structures of both alloxazine mononucleotide and alloxazine adenine dinucleotide are depicted in Figures 1 and 2.

Stern and Holiday (1), by spectrographic methods, first found that the prosthetic group of Warburg's old yellow enzyme (2), which will be considered in more detail below, was a derivative of alloxazine, and the structure of the riboflavin phosphate was finally determined independently by Kuhn (3), Karrer (4), and their collaborators. Theorell (5) demonstrated that the enzyme contained one molecule of phosphate; and later Kuhn, Rudy, and Weygand (6), by synthesizing riboflavin-5-phosphoric acid, demonstrated the position occupied by the phosphate group.

In Figure 1 the hydrogenation of the alloxazine mononucleotide is also indicated. The process of hydrogenation undoubtedly takes place in such a way that one hydrogen atom at a time is transferred from the substrate molecule to the riboflavin phosphate. The formation of a red-colored intermediate in the reduction process constitutes much of the evidence in favor of this view. Kuhn and Wagner- 


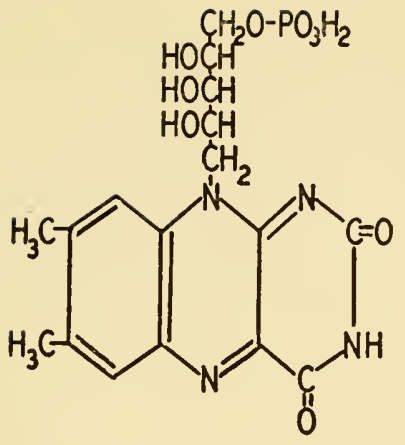

ALLOXAZINE MONONUCLEOTIDE
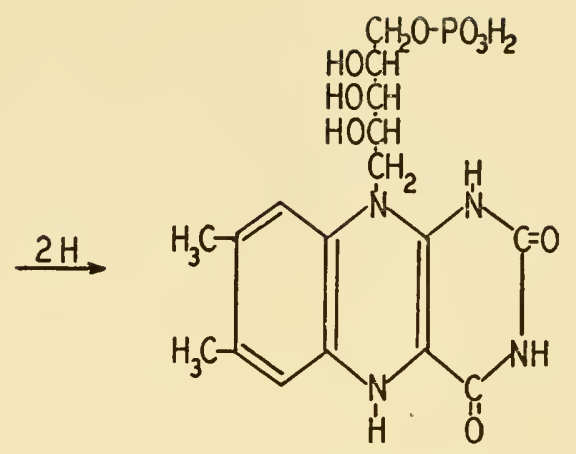

DIHYDROALLOXAZINE MONONUCLEOTIDE

FIGURE 1

Jauregg (7) made the observation that when riboflavin and also alloxazine are reduced in acid solution red-colored intermediates with absorption bands having maxima at $490 \mathrm{~m} \mu$. were formed. Haas (8) was able to demonstrate this intermediate formation with the old yellow enzyme and it has also been found in the case of

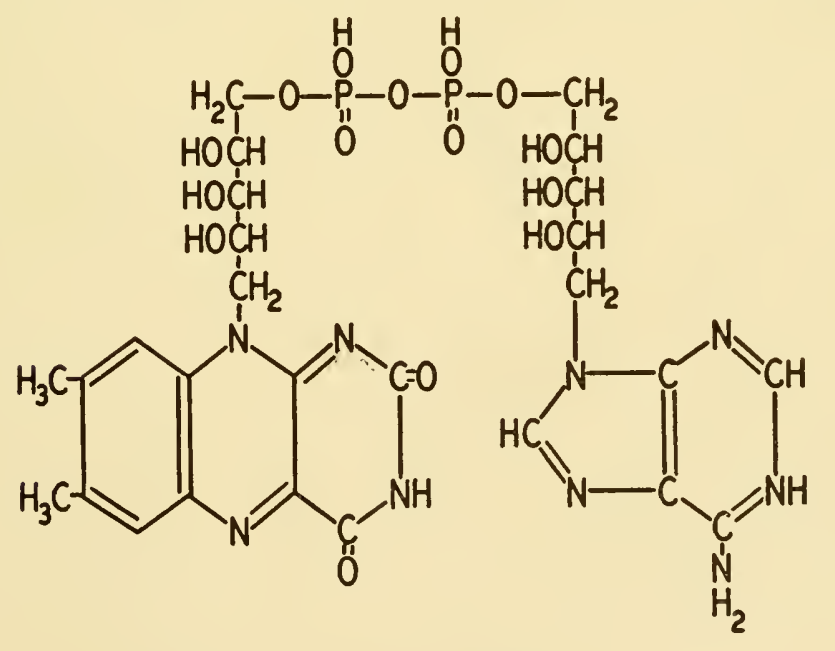

\section{ALLOXAZINE ADENINE DINUCLEOTIDE}

FIGURE 2 


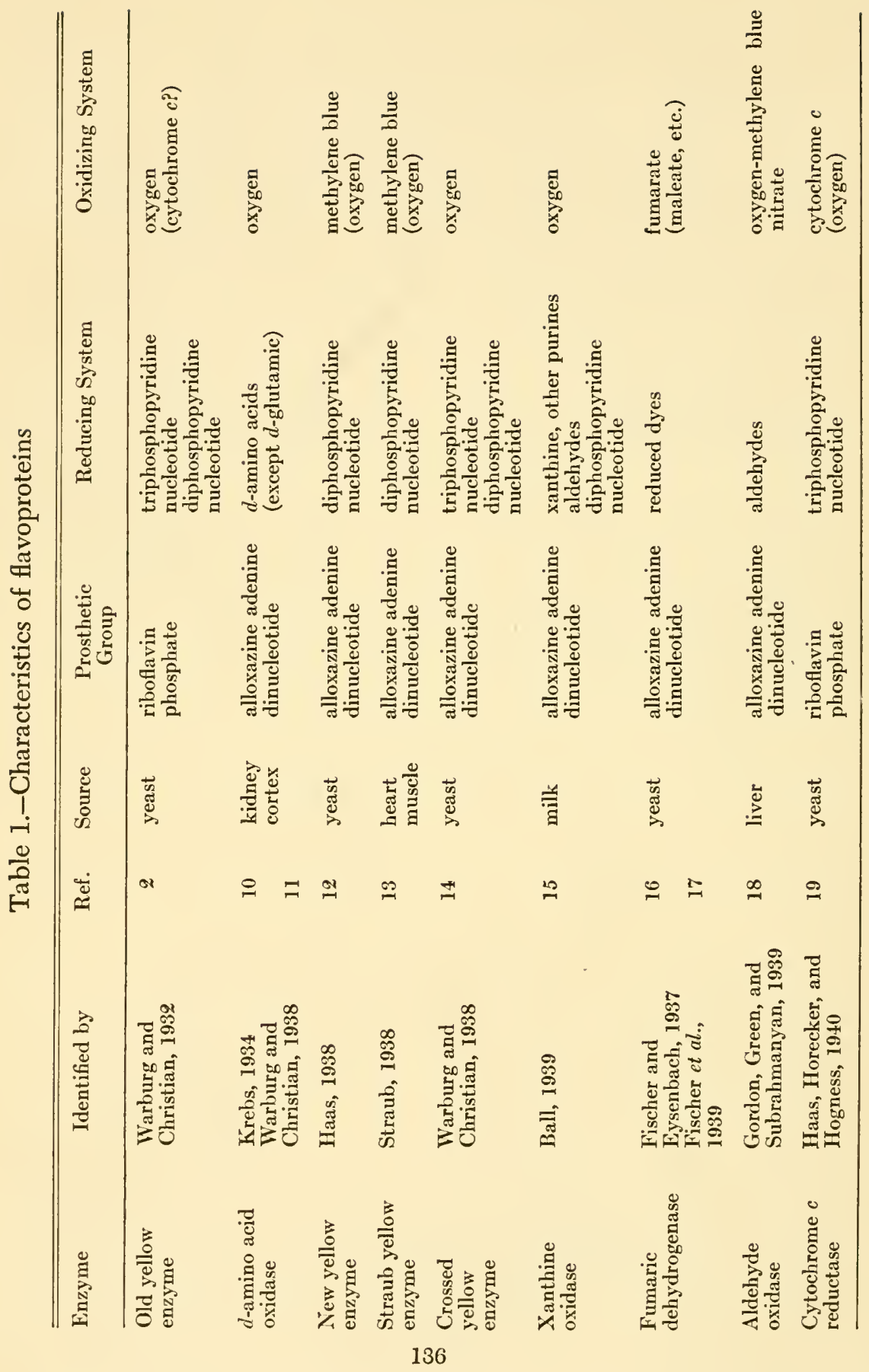


cytochrome $c$ reductase (9). In Table I the characteristics of the known and identified flavoproteins are presented in outline form.

Inasmuch as three excellent reviews of the respiratory enzymes by Kalckar (20), Green (21), and Oppenheimer and Stern (22) have recently appeared which collectively deal at length with the flavoproteins, I shall consider only the highlights that characterize them and treat them by comparison with one another. Later I shall consider in some detail that particular flavoprotein with which I am most familiar and which was isolated by my two collaborators, Haas and Horecker-cytochrome $c$ reductase. I should like to add that Mr. Haas is responsible for much of the experimental work reported by me here.

The Old Yellow Enzyme.-After Barron and Harrop (23) found that methylene blue could bring about the respiration of erythrocytes, Warburg and Christian repeated these experiments, using an extract of horse erythrocytes, with hexose monophosphate as the substrate. They were able to separate from this extract three factors necessary in the respiration process. From these three separate components, the old yellow enzyme, triphosphopyridine nucleotide, and Zwischenferment were later isolated. The old yellow enzyme was thus the first flavoprotein to be discovered. The prosthetic group of the old yellow enzyme is alloxazine mononucleotide or riboflavin phosphate.

An interesting observation that has never been explained came out of these first experiments of Warburg and Christian. Whereas it was necessary to add methylene blue to a fresh extract of horse erythrocytes to bring about respiration, the addition of the dye was not necessary when the extract had been dried and subsequently dissolved. In the latter case the old yellow enzyme presumably reacted directly with oxygen. If so, then why did not the same reaction also take place with the fresh extract?

The d-Amino Acid Oxidase.-In 1934 Krebs (10) identified both the $d$ - and the $l$-amino acid oxidases in an extract obtained from kidney cortex. He found that whereas the $l$-amino acid oxidase is inhibited by cyanide, the $d$-amino acid oxidase is not. Furthermore, when the extract is dried, the $l$-amino acid oxidase is destroyed, whereas the $d$-amino acid oxidase remains active.

Beginning with Krebs' findings, Warburg and Christian (11) isolated the $d$-amino acid oxidase and found that its prosthetic group was a dinucleotide made up of riboflavin phosphate and adenylic acid. The $d$-amino acid oxidase reacts with most of the 
$d$-amino acids, oxidizing them to the alpha-keto acids and ammonia; $d$-glutamic acid is a notable exception. Its reactivity with oxygen is greater than that of the other flavoproteins.

The New Yellow Enzyme.-The new yellow enzyme, isolated by Haas (12) immediately after the first isolation of the $d$-amino acid oxidase, was found to have associated with it, as its prosthetic group, alloxazine adenine dinucleotide. It reacts with diphosphopyridine nucleotide as does the old yellow enzyme; its reactivity toward oxygen is considerably less than that of the old yellow enzyme, but it is very active toward methylene blue.

The Straub Yellow Enzyme.-This enzyme (13) was isolated from heart muscle and in all probability is the same enzyme that Haas isolated from yeast.

The Crossed Yellow Enzyme.-Warburg and Christian (14) "synthesized" a new enzyme by having the protein moiety of the old yellow enzyme combine with alloxazine adenine dinucleotide rather than with the mononucleotide in combination with which it was isolated from yeast. The properties of this crossed enzyme are similar to those of the old yellow enzyme.

Xanthine Oxidase.-This enzyme, isolated in a purified state by Ball (15), catalyzes the oxidation of the purines, particularly xanthine, the aldehydes, and diphosphopyridine nucleotide (24), by oxygen. Its prosthetic group is alloxazine adenine dinucleotide. Since its exact nature has not yet been elucidated, it is possible that this enzyme contains components not yet accounted for. As defined by its activity toward aldehydes, it was once known as "Schardinger's Enzyme." It was first identified in milk by Morgan, Stewart, and Hopkins (25).

Fumaric Dehydrogenase.-This enzyme catalyzes the reduction of fumaric acid by one of several leuco dyes, the products of the reaction being succinic acid and the oxidized or colored dye. The activity of the enzyme is measured by the rate at which the color appears. It was discovered by Fischer and Eysenbach (16) in 1937, and in 1939 Fischer, Roedig, and Rauch (17) purified it further by electrophoresis. No physiological reducing agent has been found with which this enzyme is active.

Aldchyde Oxidase.-Like xanthine oxidase, this enzyme catalyzes the oxidation of aldehydes but differs from the xanthine oxidase in that it does not catalyze the oxidation of xanthine. It was isolated in 1939 from liver by Gordon, Green, and Subrahmanyan (18).

Cytochrome c Reductase.-This flavoprotein acts as the inter- 
mediary link between cytochrome $c$ and triphosphopyridine nucleotide. It will later receive special attention.

A glance at Table 1 shows clearly that many of these flavoproteins are closely associated with either disphospho- or triphosphopyridine nucleotide. They therefore constitute a link in the hydrogen transport system. Nor is it surprising that so many of them react with molecular oxygen. In fact, we should expect all of them to be autoxidizable, at least to a small extent, for all of them are presumably dissociable into the prosthetic group and a protein, and the prosthetic group itself is autoxidizable. In some cases, however, the prosthetic group, when attached to the protein, is very probably also autoxidizable. If this were not true, we might expect that those flavoproteins which dissociate to the greatest extent, i.e., those having the largest dissociation constants, would have the greatest rate of autoxidation. There is evidence that those flavoproteins with the greater dissociation constants are more autoxidizable, but it is not conclusive. In making such a comparison we must compare with each other only those flavoproteins having the same prosthetic group and under such conditions that the oxidation of the flavoprotein is the rate-determining step.

Each of the flavoproteins dissociates to a different extent into its prosthetic group and its protein moiety. The degree of dissociation of any flavin nucleotide-protein complex is determined by a procedure which involves first splitting the complex into its two constituent parts and separating them. By adding increasing amounts of the prosthetic group to a fixed amount of protein and choosing conditions such that the reaction velocity is proportional to the amount of complex formed, it is then possible to determine the dissociation constant from the well-known Michaelis-Menten equation, or some modification of it. This equation states that

$$
\frac{V}{V_{m}}=\frac{(S)}{K_{1}+(S)}
$$

in which $V$ is the velocity, $V_{m}$ the maximum velocity (when all protein is in the form of a complex), (S) is the total concentration of the prosthetic group, and $K_{i}$ is the dissociation constant. When $V / V_{m}=0.5$, then $K_{1}$ is equal to (S). This equation is valid only when the dissociation constant is so high or the concentration of the protein so low that the amount of the prosthetic group bound to the protein is small as compared with the total amount in the solution. Under these conditions the concentration of the uncombined pros- 
thetic group and the total concentration (combined and uncombined) may be regarded as the same. If this assumption cannot be made, then a somewhat more complicated equation must be used (see ref. 19).

The splitting of the flavoprotein has been accomplished in two ways. Theorell (26) separated the riboflavin phosphate and the protein by a 72-hour dialysis against a dilute acid solution. By adding the riboflavin phosphate to the protein remaining after the dialysis, the original activity was restored. Warburg and Christian (27) later developed a simpler and more rapid method in which the solution containing the flavoprotein is acidified in the presence of large concentrations of ammonium sulfate. In this solution the flavoprotein is split and the protein is precipitated. The ammonium sulfate protects the protein moiety against denaturation.

The known dissociation constants, determined in the manner previously outlined, are presented in Table 2.

Table 2.-Dissociation constants

\begin{tabular}{lcc}
\hline \multicolumn{1}{c}{ Enzyme } & Dissociation constant & Reference \\
\hline Amino acid oxidase & $250 \times 10^{-9}$ & 28 \\
Old yellow enzyme & $60 \times 10^{-9}$ & 2 \\
New yellow enzyme & $27 \times 10^{-9}$ & 12 \\
Cytochrome $c$ reductase & $1 \times 10^{-9}$ & 19 \\
\hline
\end{tabular}

In comparing the activities of various enzymes, several factors must be taken into account. These are (1) the concentrations of the reacting substances, (2) the affinity of the enzyme for the reacting substrate molecules, and (3) the absolute reaction velocity of the reactants when in the form of the protein-substrate complex. The last two of these factors determine the intrinsic activity of the enzyme, but in determining how great a role any enzyme plays in a given cell, the concentrations are of prime importance.

All the evidence indicates that in every case substrate and enzyme form a complex before reaction sets in. As an example we may consider the reaction between reduced triphosphopyridine nucleotide $\left(\mathrm{TPNH}_{2}\right)$ and oxidized cytochrome $c$ reductase $(\mathrm{CR})$.

$$
\mathrm{TPNH}_{2}+\mathrm{CR}=\mathrm{TPN}+\mathrm{CRH}_{2}
$$

A complex is first formed and the velocity of the reaction is proportional to the concentration of this complex. 
The equations representing the dissociation and the equilibrium expression or dissociation constant for the formation of the complex are as follows:

$$
\begin{aligned}
& \text { Complex }=\mathrm{CR}+\mathrm{TPNH}_{2} \\
& K_{1}=\frac{(\mathrm{CR})\left(\mathrm{TPNH}_{2}\right)}{(\text { Complex })}
\end{aligned}
$$

The velocity of the reaction is proportional to the concentration of the complex.

$$
v=K_{r} \text { (Complex) }
$$

Since

$$
(\mathrm{CR})_{\text {total }}=(\mathrm{CR})+(\text { Complex })
$$

$$
v=\frac{K_{r}\left(\mathrm{TPNH}_{2}\right)(\mathrm{CR})_{\text {total }}}{K_{1}+\left(\mathrm{TPNH}_{2}\right)}
$$

This equation is essentially the Michaelis-Menten equation. I am considering it in some detail only because I wish to use it in reference to the determination and definition of enzyme activity.

In the above derivation I have assumed that the products of the reaction have no inhibitory influence, or that the conditions are such that the concentrations of the products are negligibly small. The velocity plotted against the concentration of dihydrotriphosphopyridine nucleotide is given in Figure 3. This curve is strictly in accord with equation 7 .

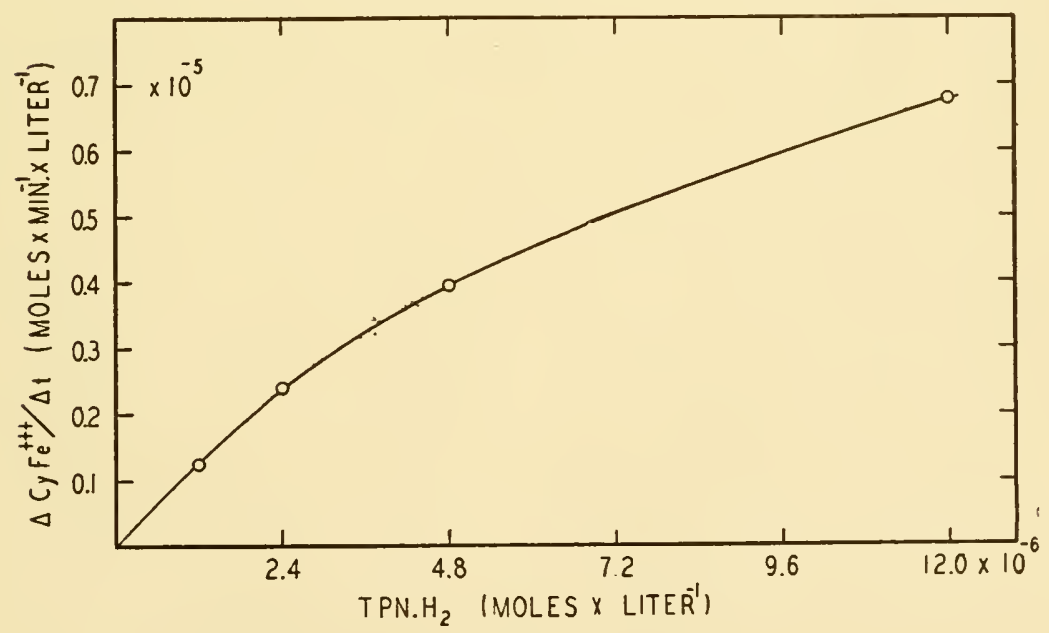

FIGURE 3 
The magnitude of $K_{r}$ denotes the velocity of the reaction within the complex, and the value of $K_{1}$ gives a measure of the concentration of the complex. Thus in more dilute solutions of the substrate, when the enzyme is not saturated, both $K_{r}$ and $K_{1}$ determine the activity-the larger $K_{r}$ and the smaller $K_{1}$, the more active is the enzyme. Unfortunately not enough data are present to permit of so specific a comparison of enzymatic activities. An approximation, however, can be made. If the concentration of the substrate, $\mathrm{TPNH}_{2}$ in this case, is very small as compared with $K_{1}$, then equation 7 reduces to:

or

$$
\begin{aligned}
& v=\frac{K_{r}}{K_{1}}\left(\mathrm{TPNH}_{2}\right)(\mathrm{CR})_{\text {total }} \\
& v=K^{\prime}\left(\mathrm{TPNH}_{2}\right)(\mathrm{CR})_{\text {tota } 1}
\end{aligned}
$$

Under these conditions the velocity is proportional to the concentration of the $\mathrm{TPNH}_{2}$ (lower left part of curve), and $K^{\prime}$, which is approximately equal to $K_{r} / K_{1}$ is a measure of the activity-the larger the velocity constant $K_{r}$ and the smaller the dissociation constant $K_{1}$, the more active is the enzyme. Neither $K_{r}$ nor $K_{1}$ are affected by the concentration of the substrate or enzyme, whereas the turnover number may be.

In more concentrated solution, i.e., when the enzyme is saturated with substrate, only $K_{r}$ determines the activity $\left(v=K_{r}(\mathrm{CR})_{\text {total }}\right)$. Thus the significance of the dissociation constant is apparent only when the substrate is present in low concentration-a low dissociation constant enhances the activity. On the basis of this criterion of activity, a comparison between the activities of the various flavoproteins for low concentrations of substrate is given in Table 3.

\begin{tabular}{|c|c|c|c|c|}
\hline Enzyme & $\begin{array}{l}\text { Prosthetic } \\
\text { group }\end{array}$ & $\begin{array}{c}\text { Reaction with } \\
\text { dihydrotri- } \\
\text { phospho- } \\
\text { pyridine } \\
\text { nucleotide } \\
K^{\prime}\end{array}$ & $\begin{array}{c}\text { Reaction } \\
\text { with } \\
\text { oxygen } \\
K^{\prime}\end{array}$ & $\begin{array}{c}\text { Reaction } \\
\text { with cyto- } \\
\text { chrome } c \\
K^{\prime}\end{array}$ \\
\hline Old yellow enzyme & $\begin{array}{l}\text { alloxazine mono- } \\
\text { nucleotide }\end{array}$ & $6 \times 10^{3}$ & $10.0 \times 10^{4}$ & $0.3 \times 10^{5}(?)$ \\
\hline New yellow enzyme & $\begin{array}{l}\text { alloxazine adenine } \\
\text { dinucleotide }\end{array}$ & $22 \times 10^{6}$ & $1.4 \times 10^{4}$ & 0 \\
\hline $\begin{array}{l}\text { Cytochrome } c \text { reduc- } \\
\text { tase }\end{array}$ & $\begin{array}{l}\text { alloxazine mono- } \\
\text { nucleotide }\end{array}$ & $170 \times 10^{6}$ & $0.8 \times 10^{4}$ & $53,000 \times 10^{5}$ \\
\hline
\end{tabular}

Table 3.-Specific reaction velocities at $25^{\circ} \mathrm{C}$. 
Theorell (29) found that reduced old yellow enzyme reacted to a very small extent with cytochrome $c$. In view of the fact that the activity of cytochrome $c$ reductase toward cytochrome $c$ is more than 100,000 times greater than that of the old yellow enzyme in low concentrations, it is conceivable that Theorell's sample of the old yellow enzyme contained a trace of the reductase.

My two collaborators, Haas and Harrer (9), have found that when the cytochrome $c$ reductase is kept at $0^{\circ} \mathrm{C}$. for four weeks the enzyme becomes partially denatured. Its activity, as measured by the second-order velocity constant, with respect to triphosphopyridine nucleotide and cytochrome $c$, decreases 91 per cent, and its activity toward triphosphopyridine nucleotide and oxygen decreases 36 per cent. These experiments indicate that Warburg's old yellow enzyme is not denatured cytochrome $c$ reductase, for if it were, one would expect the activity of the reductase toward oxygen to increase with this specific deactivation, since the activity of Warburg's old yellow enzyme toward oxygen is greater than that of cytochrome $c$ reductase.

Only two of the flavoproteins are oxidized by physiological substrates other than oxygen, namely, fumaric dehydrogenase and cytochrome $c$ reductase. But since it has not been demonstrated that fumaric dehydrogenase is reduced by physiological substrates, cytochrome $c$ reductase constitutes the only known link between the pyridine protein system (specifically triphosphopyridine nucleotide) and the cytochromes in the hydrogen transport system. SzentGyörgyi (30), on the basis of the catalytic effect of small amounts of dicarboxylic acids, succinic-fumaric and malic-oxalacetic, on tissue respiration, and on the basis of the finding that dihydro-alloxazine can be oxidized enzymatically by fumaric acid, postulated that the succinate-fumarate couple served as the missing link between old yellow enzyme and cytochrome $c$. However, no direct experimental evidence has been produced to substantiate this viewpoint. The fact that cytochrome $c$ reductase, a very active flavoprotein itself, acts in this capacity has obviated the necessity for a link between the old or new yellow enzymes and the cytochrome system.

The hydrogen transport system (or one branch of it), as we now definitely know it, consists of the following series of reactions:

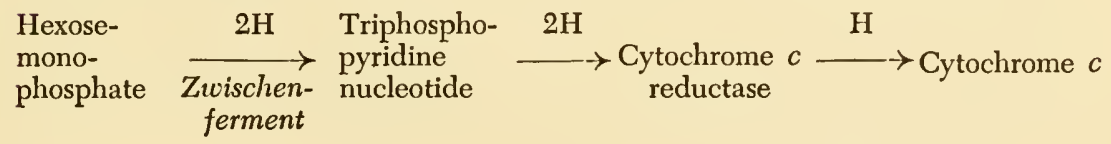


The cytochrome $c$ is oxidized by oxygen through the intermediation of an enzyme or enzymes known as cytochrome oxidase, and by hydrogen peroxide with cytochrome $c$ peroxidase as the catalyst (31). The cytochrome $c$ oxidase, or one component of it, is probably Warburg's oxygen-carrying ferment, but it has not yet been definitely identified. Nor do we yet understand the whole mechanism involved in the oxidation of cytochrome $c$.

The test for determining the relative concentration of the cytochrome $c$ reductase involves all these components. If oxidized cytochrome $c$ is placed in a solution containing all the other components and if the concentration of the hexose monophosphate is in excess, the cytochrome $c$ will be reduced, and the rate at which it is reduced will depend upon the concentrations of the other components: Zwischenferment, triphosphopyridine nucleotide and the cytochrome $c$ reductase. The concentrations of all substances can be so adjusted that the logarithmic rate of reduction of the cytochrome $c$ will be proportional to the concentration of the reductase.

$$
\frac{-\mathrm{d} \log \left(\mathrm{CyFe}^{+++}\right)}{\mathrm{dt}}=K(\mathrm{CR})
$$

In this equation $\left(\mathrm{CyFe}^{+++}\right)$represents the concentration of the oxidized cytochrome $c$ and (CR) the concentration of the cytochrome $c$ reductase. The equation is an empirical one.

It is easy to demonstrate that all components of the system must be present before the cytochrome $c$ will be reduced. In the reduced state cytochrome $c$ displays three bands with maxima at about 410 , 520 , and $550 \mathrm{mp}$, and any one of these bands may be used to determine the rate of reduction of the cytochrome. For most purposes the band at $550 \mathrm{~m} \%$ is most convenient for analytical purposes, although for very dilute solutions of cytochrome $c\left(10^{-7} \mathrm{M}\right)$ a wave length of 418 is used. As the cytochrome $c$ is reduced, these bands appear. In the following spectroscopic demonstration, the general method employed in this test is illustrated. If all components except the reductase are added to the buffered solution in the absorption cell, no reduction of the cytochrome $c$ takes place until a solution of the reductase is added to complete the chain of enzymatic reactions.

Spectroscopic demonstration: The apparatus consists of a simple focusing illuminating lantern (Central Scientific Company) slightly modified to hold an absorption cell $2.5 \mathrm{~cm}$. in diameter and $2 \mathrm{~cm}$. thick. A lamp with a ribbon type filament is used, and the filament, turned edgewise, is used as the slit. By placing a transmission grating in the light path a spectrum is projected on the wall. 
Then the cell is filled with buffer, and when all components of the reducing system except the reductase are placed before the filament, no bands appear. However, when the reductase is added, the two visible bands of cytochrome $c$ gradually appear as intense broad black lines. The cytochrome is then reduced.

To oxidize the cytochrome $c, \mathrm{H}_{2} \mathrm{O}_{2}$ is added. No reaction occurs, i.e., the black bands do not disappear, until the enzyme cytochrome $c$ peroxidase is added. Upon the addition of this latter enzyme, the two bands disappear and reappear again as soon as the $\mathrm{H}_{2} \mathrm{O}_{2}$ is completely reduced.

Not only can this system be used to determine the concentration of cytochrome $c$ reductase, but it can serve as an analytical method for the determination of any one of the constituents. In fact, my two collaborators, Haas and Harrer (9), have only recently worked out the conditions necessary for a relatively simple determination for triphosphopyridine nucleotide. By using glucose and adenosine triphosphate instead of hexose monophosphate, this system should serve as a method for determining the relative concentrations of either mutase, the enzyme that converts glucose-1-phosphate to glucose-6-phosphate, or hexokinase, the enzyme that directly phosphorylates glucose-6-phosphate.

Haas and Harrer (9) have also made very accurate measurements on the rate of oxidation of the reductase by cytochrome $c$ and the rate of reduction by dihydrotriphosphopyridine nucleotide under a great variety of conditions, and as a result we have demonstrated by these kinetic measurements that the reductase forms complexes with both the dihydrotriphosphopyridine nucleotide and with the cytochrome $c$. The approximate first-order reaction velocities have been determined, as have also the heat of dissociation of the complexes and the energy of activation of the first-order reactions. The tentative results of these studies are embodied in Table 4 . The values given in the table are approximations. Although the data

Table 4.-Properties of cytochrome $c$ reductase

\begin{tabular}{|c|c|c|c|c|}
\hline Reaction & $\begin{array}{l}\text { Dissociation } \\
\text { constant of } \\
\text { complex with } \\
\text { reductase } 25^{\circ}\end{array}$ & $\begin{array}{c}\text { First-order } \\
\text { velocity } \\
\text { constant } 25^{\circ}\end{array}$ & $\begin{array}{c}\text { Heat of } \\
\text { dissociation }\end{array}$ & $\begin{array}{l}\text { Energy of } \\
\text { activation }\end{array}$ \\
\hline $\begin{array}{l}\text { Reduction of reduc- } \\
\text { tase by } \mathrm{TPNH}_{2}\end{array}$ & $\begin{array}{l}1 \times 10^{-5} \\
\text { moles per liter }\end{array}$ & $\begin{array}{l}\text { ca. } 2000 \\
\min ^{-1}\end{array}$ & $2 \mathrm{~kg}$. cal. & $12 \mathrm{~kg}$. cal. \\
\hline $\begin{array}{c}\text { Oxidation of reduc- } \\
\text { tase by } \mathrm{CyFe}^{++}\end{array}$ & $\begin{array}{c}7 \times 10^{-8} \\
\text { moles per liter }\end{array}$ & $\begin{array}{l}\text { ca. } 2000 \\
\min ^{-1}\end{array}$ & $8 \mathrm{~kg}$. cal. & $17 \mathrm{~kg} . \mathrm{cal}$. \\
\hline
\end{tabular}


are at hand, the final calculations (which involve a series of approximations) have not been made. The dihydrotriphosphopyridine nucleotide forms a stable complex with the reductase, but the complex between the reductase and the cytochrome $c$ is a more stable one. In this latter case the complex is one in which both components are proteins; the cytochrome $c$ reductase, with a molecular weight of about 75,000 , combines with cytochrome $c$, with a molecular weight of 13,000 . In all probability both the dihydrotriphosphopyridine nucleotide and the cytochrome $c$, as well as the riboflavin phosphate, are simultaneously attached to the same protein molecule.

This picture of the oxidation-reduction complex inspires speculation with respect to the manner in which the hydrogen atoms are transferred from the dihydrotriphosphopyridine nucleotide to the riboflavin phosphate constituents of the complex. Because of the complexity of the molecules involved, it is difficult to imagine the "active" positions of each of them approaching close enough for a direct hydrogen transfer. The most likely mechanism seems to be that of ionization, with a hydrogen ion dissociating into the solution, this process followed by an electron transfer from one molecule to the other, and this in turn followed by an attachment of another hydrogen ion from the solution to the new position of the electron. In the oxidation or reduction of cytochrome $c$ we already regard the change taking place as electronic.

Cytochrome $c$ reductase bridges one gap in the hydrogen transport system-that between cytochrome and triphosphopyridine nucleotide. The other gap, between the cytochrome system and diphosphopyridine nucleotide, is still open, although a beginning toward the solution of this problem has been made. My two collaborators, Altschul and Persky (32), have found a soluble protein in yeast that is capable of acting as an intermediate in the reduction of cytochrome $c$ by dihydrodiphosphopyridine nucleotide. It is soluble; like other proteins it is precipitated by ammonium sulfate, acetone, and alcohol; it is heat-labile, and can be dialyzed without great loss of activity. It is not reactive toward triphosphopyridine nucleotide. Since its enzymatic function is so nearly like that of cytochrome $c$ reductase, it, too, is probably a flavoprotein, although there is not yet any direct evidence to this effect.

Of the flavoproteins which react with either dihydrodiphosphopyridine nucleotide or dihydrotriphosphopyridine nucleotide, only Warburg's old yellow enzyme and cytochrome $c$ reductase react directly, and to any appreciable extent, with physiological oxidizing 
agents; the reaction between Haas's new yellow enzyme or Straub's yellow enzyme and oxygen is very slow, unless methylene blue is used as a "carrier." Possibly carriers other than methylene blue exist in respiring cells. Xanthine oxidase and aldehyde oxidase react with reducing agents which, while important, do not contribute greatly to the production of energy. Lipmann (33), using the Warburg separation technique, split from a soluble fraction obtained from Bacterium Delbrückii (Lactobacillus delbrückii) a protein portion which was active as pyruvic acid oxidase only when both thiamine pyrophosphate and alloxazine adenine dinucleotide were added. From this result he postulated that a yellow enzyme possibly oxidizes thiamine pyrophosphate. Green, Knox, and Stumpf (34) have recently reported the finding of another yellow enzyme, the function of which has not yet been determined.

Formerly it was assumed that cytochrome $b$, because of its potential, acted as one of the intermediaries between the flavoproteins and cytochrome $c$, but the discovery of cytochrome $c$ reductase has obviated the necessity for any such intermediary, although in the intact cell it may act as such (two or more paths of oxidation may be in operation).

At present nine flavoproteins are known, not all of which react with both the oxidizing and the reducing agents which are present in the living cell; the discovery of more or these important enzymes will undoubtedly follow. The relatively large riboflavin content of the liver and the kidney and the multiple and complicated biochemical functions of these organs alone indicate that we might expect to find many more members of this class of enzymes.

\section{REFERENCES}

1. Stern, K. G., and Holmay, E. R., Ber., 67, 1104, 1442 (1934).

2. Warburg, O., and Christian, W., Biochem. Z., 254, 438 (1932); 263, 228 (1933); 287, 291, 440 (1936).

3. Kuhn, R., et al., Ber., 66, 1034 (1933); 68, 1765 (1935); 69, 1557 (1936).

4. Karrer, P., Helv. Chim. Acta, 18, 69, 72, 426 (1935); Ber., 68, 216 (1935).

5. Theonell, H., Biochem. Z., 272, 155 (1934).

6. Kuhn, R., Rudy, H., and Weygand, F., Ber., 69, 2034 ( 1936 ).

7. Kuhn, R., and Wagner-Jauregg, Th., Ber., 67, 361 (1934).

8. HaAs, E., Biochem. Z., 290, 291 (1937).

9. HaAs, E., Harrer, C., and Hogness, T. R. (unpublished).

10. Krebs, H. A., Biochem. J., 29, 1620 (1935).

11. Warburg, O., and Christian, W., Biochem. Z., 296, 294; 298, 150 (1938).

12. HaAs, E., Biochem. Z., 298, 378 (1938).

13. Straub, F. B., Biochem. J., 33, 787 ( 1939 ).

14. Warburg, O., and Christian, W., Biochem. Z., 298, 368 (1938).

15. Ball, E. G., J. Biol. Chem., 128, 51 (1939). 
16. Fischer, F. G., and Eysenbach, H., Ann., 530, 99 (1937).

17. Fischer, F. G., Roedig, A., and RaUCH, K., Naturwissenschaften, 27, 197 (1939).

18. Gordon, A. H., Green, D. E., and Subrammanyan, V., Biochem. J., 34, 764 (1940).

19. HaAs, E., Horecker, B. L., and Hogness, T. R., J. Biol. Chem., 136, 747 (1940).

20. Kalckar, H. M., Chem. Rev., 28, 72 (1941).

21. Green, D. E., Mechanisms of Biological Oxidations (Cambridge University Press, 1940).

22. Oppenheimer, C., and Stern, K. G., Biological Oxidation (W. Junk, The Hague, 1939).

23. Barron, E. S. G., and Harrop, G. A., J. Exp. Med., 48, 207 (1928), J. Biol. Chem., 79, 65 (1928).

24. Ball, E. G., and Ramsdell, P. A., J. Biol. Chem., 131, 767 (1939).

25. Morgan, E. J., Stewart, C. P., and Hopkins, F. G., Proc. Roy. Soc. (London), B 94, 109 (1922).

26. Theorell, H., Biochem. Z., 275, 37 (1934).

27. Warburg, O., and Christian, W., Biochem. Z., 296, 294 (1938).

28. Warburg, O., and Christian, W., Biochem. Z., 295, 261; 298, 150 (1938).

29. Theorell, H., Biochem. Z., 288, 317 (1936).

30. Szent-Györgyi, A., Z. physiol. Chem., 249, 211 (1937).

31. Altschul, A. M., Abrams, R., and Hogness, T. R., J. Biol. Chem., 136, 777 (1940).

32. Altschul, A. M., Persky, H., and Hogness, T. R., Science, 94, 349 ( 1941 ).

33. Lipmann, F., Nature, 143, 436 (1939).

34. Green, D. E., Knox, W. E., and StumpF, P. K., J. Biol. Chem., 138, 775 (1941). 


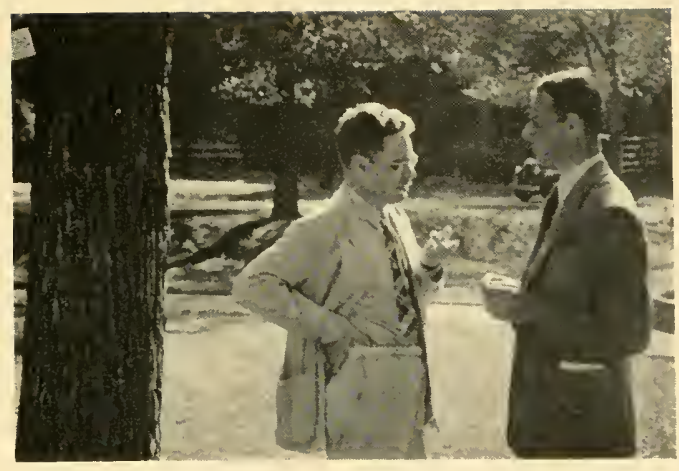

WILSON AND KALCKAR:

"The manuscript deadline is . . ."

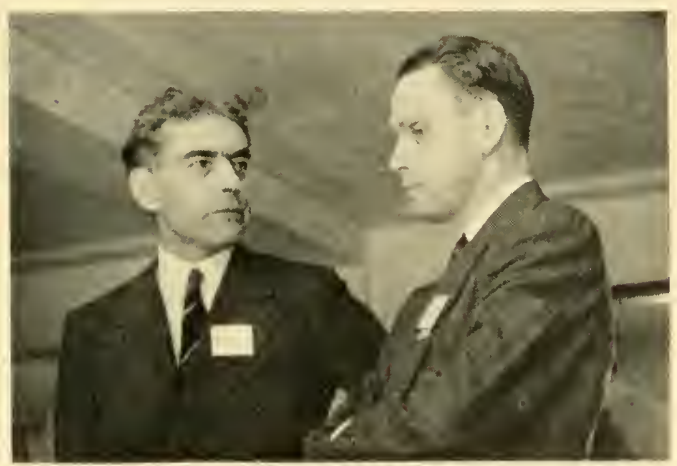

Hogness and Elvehjen: "The respiratory enzyme, alloxazineadenine dinucleotide, . . ." The vitamin, riboflavin, ..."

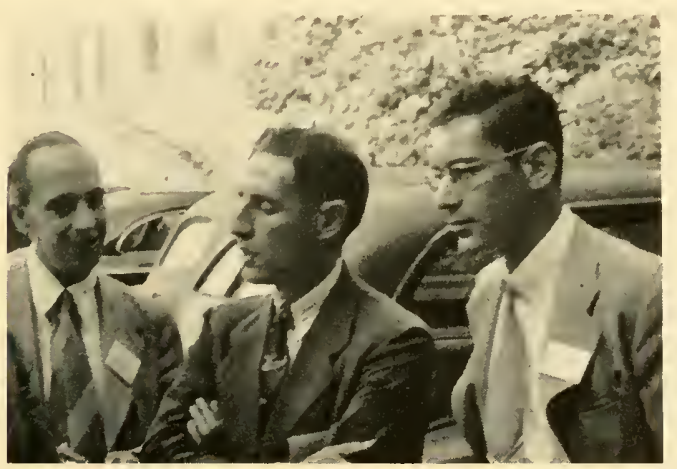

Ochod, Wood, and Carson: "With heavy carbon . . ."

"But with radioactive carbon .. ."

COMMENTS OFF THE RECORD 



\section{Cytochromes}

\section{ELMER STOTZ}

Harvard University*

7 HE SUBJECT OF THE iron-containing cellular respiration catalysts 1 has demanded the attention of many biochemists. One obvious reason for this is the fact that the cytochromes are so readily detectable by spectroscopic means that their wide distribution and hence their apparent importance were early recognized $(1,2)$. With increasing knowledge of cellular respiration the unique and fundamental position of the cytochromes in the respiratory scheme has been emphasized more and more. Although the various substrates of respiration may require many enzymes, coenzymes, and mediators, the individual pathways appear to converge at the cytochrome system. It is through the ferrous to ferric change of this system that the electrons of the ultimate substrate, hydrogen, come to terms with the ultimate oxidant, oxygen.

In proportion to its importance in respiration, perhaps less is known of the cytochromes than of other respiratory components. Cytochrome $c$ has been isolated, but the peculiar linkage of its prosthetic group with the protein is not yet fully understood. Cytochromes $a$ and $b$ still remain bands in the absorption spectrum of tissues with little appreciation of their function. Finally, the allimportant cytochrome oxidase is still little more than the insoluble ground residue of tissues.

\section{Properties of the Cytochrome Components}

Cytochrome c.-Cytochrome $c$ appears to be quantitatively the most important of the three cytochrome constituents. It was defined spectroscopically by Keilin $(2,3)$ as that component which in the reduced state has an alpha-band at $5500 \mathrm{~A}$. and a beta-band at $5200 \mathrm{~A}$. It was first isolated in apparently pure form by Theorell (4) in 1936 by a dilute sulfuric acid extraction of defatted beef heart muscle; isolation was followed by ammonium sulfate precipitation, barium sulfate adsorption, acetone precipitation, and finally adsorption on cellophane. Shortly thereafter Keilin and Hartree (5)

- Contribution from the McLean Hospital, Waverly, Massachusetts, and the Harvard Medical School, Boston. 
described a simpler method of preparation involving a trichloracetic acid extraction of the ground muscle, ammonium sulfate fractionation, and trichloracetic precipitation. Both groups of workers obtained a product which had the same absorption bands as the cytochrome $c$ of the intact or phosphate-extracted muscle, and which contained 0.34 per cent iron, resisted further fractionation, and was therefore considered pure. Later Theorell and Akesson (6) obtained by electrophoretic means a product containing as high as 0.43 per cent iron. It would be of interest to know whether this product displayed an equal increase in catalytic activity over the 0.34 per cent iron product.

Zeile and Reuter (7) calculated a molecular weight of 18,000 for cytochrome $c$ on the basis of its hemin content. Theorell (4), from a study of its diffusion and sedimentation, determined a molecular weight of 16,500 . The iron content of 0.34 per cent also yields an equivalent weight of 16,500 . The isoelectric point of cytochrome $c$ is approximately at $\mathrm{pH} 9.8$ (4).

Ferric cytochrome $c$ is readily reduced by a variety of agents, such as hydrosulfite, ascorbic acid, cysteine, adrenalin, hydroquinone, $p$-phenylenediamine, and many leuco dyes, as well as by certain physiological reducing systems to be discussed later.

Ferro-cytochrome $c$ is essentially non-autoxidizable in neutral solution, the slow rate being largely inhibited by small amounts of cyanide (8), indicating heavy metal catalysis. It is readily oxidized by ferricyanide and aerobically by oxidase preparations. Below $\mathrm{pH}$ 4.0 and above 11.0 the spectrum changes and the substance becomes autoxidizable.

A neutral solution of cytochrome $c$ can be boiled, and upon cooling the original spectrum and catalytic properties return. Cytochrome $c$ is likewise stable to dilute acid. It is stable to 0.1 normal potassium hydroxide, but 1.0 normal alkali produces an irreversible change in the spectrum. The substance is then autoxidizable and forms a lightsensitive carbon monoxide compound (9).

According to Keilin (10), cytochrome $c$ does not appear to combine with hydrogen sulfide, hydrogen cyanide, sodium azide, or hydroxylamine, nor, according to Stern (11), with carbylamine. Ferri-cytochrome $c$ does, however, form a compound with nitric oxide (12). Keilin (13) was unable to detect any change in the spectrum of the $c$ component in the presence of carbon monoxide except in solutions whose $\mathrm{pH}$ was above 13.0. Altschul and Hogness (14), using an accurate photoelectric spectrophotometer (15) with a 
narrow slit, have, however, found evidence of a ferro-cytochromecarbon monoxide compound throughout the entire $\mathrm{pH}$ range. The change in spectrum was reversible; that is, the carbon monoxide could be removed by nitrogen. The carbon monoxide compound was light-sensitive. Keilin and Hartree (10) attribute this finding to the presence of denatured cytochrome or other hematin compounds. At neutral $\mathrm{pH}$ they were able to liberate and measure manometrically only 10 per cent of the theoretical amount of carbon monoxide that should combine with reduced cytochrome.

Potter (16) concluded that cyanide also, contrary to popular belief, forms a complex with ferri-cytochrome $c$. This conclusion was based not only on the fact that a change in the spectrum was detected but also upon studies on the enzymatic reduction of cytochrome $c$. Since the spectral shift is small, as with carbon monoxide, Potter questions whether one can safely conclude from simple spectroscopic observation that a given inhibitor has not reacted with cytochrome.

Considerable gains have been made in determining the structure of the prosthetic group of cytochrome $c$ and how this might explain the peculiar stability of the heme-protein linkage. Hill and Keilin (17) obtained a porphyrin by hydrochloric acid and sulfur dioxide treatment of cytochrome $c$ which, unlike most porphyrins, was soluble in water. Zeile and Piutti (18), in extensive synthetic work, were able to introduce various nitrogen bases into the unsaturated side chains of protoporphyrin and obtain porphyrins whose solubility was similar to that obtained from cytochrome $c$. When iron was introduced into some of these compounds, they showed the characteristic cytochrome $c$ absorption band at $5500 \mathrm{~A}$. Later Zeile and Reuter (7) isolated hematoporphyrin from a hydrobromic-acetic acid degradation of cytochrome $c$. Theorell (19) isolated a sulfurcontaining porphyrin and postulated that the vinyl groups of the hemin are linked to amino acids of the protein by thio-ether bonds. Upon demonstrating later that such a porphyrin could arise by condensation of hemato-porphyrin with cysteine during the course of the cytochrome hydrolysis, Theorell (20) explained that either nitrogen or oxygen as well as sulfur might form the connecting link. Zeile and Meyer (21) offer support to the sulfur-bridge theory in obtaining the sulfur-containing porphyrin under conditions of hydrolysis in which a condensation of porphyrin with free cysteine would be very unlikely. A tentative structure of cytochrome $c$ is illustrated in Figure 1. 
Cytochrome $c$ has, as compared with other biological systems, a very high oxidation-reduction potential. As early as 1932 Coolidge (22), using an impure preparation from yeast, reported a potential. The rather unsatisfactory potential with the electrode could be stabilized with hydroquinone. By adding oxidants or reductants to a point where the spectrum of the cytochrome changed, an $E_{0}{ }^{\prime}$ of $+0.260 \mathrm{v}$. at $\mathrm{pH} 7.0$ was recorded. Lower values were obtained at $\mathrm{pH}$ 5.0. In 1934 Green (23) determined the potential of an impure

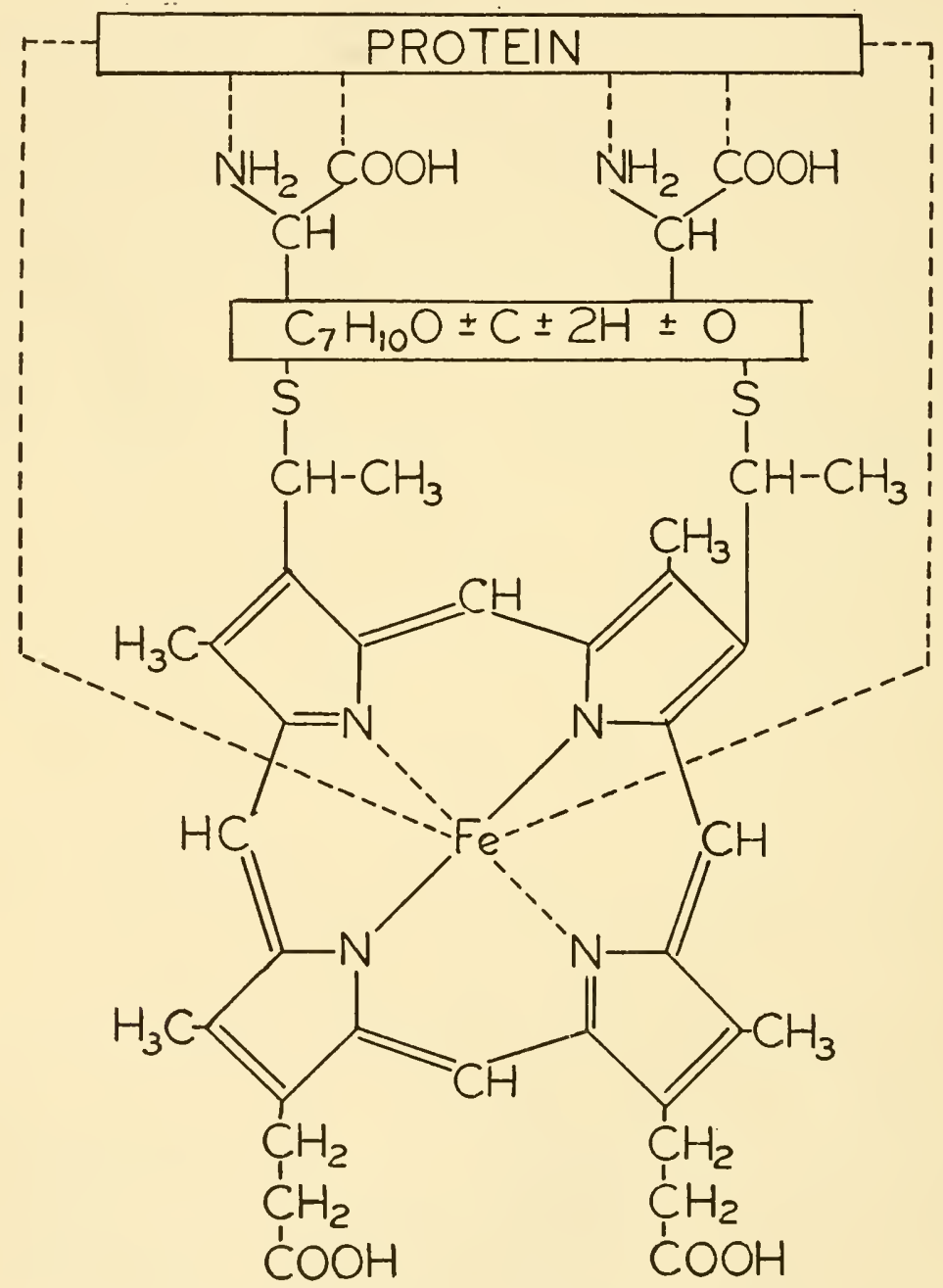

Figure 1.-Structure of cytochrome $c$ 
yeast cytochrome $c$, but found a much lower value of $\mathrm{E}_{0}{ }^{\prime}=+0.127$ v. between $\mathrm{pH} 4.6$ and 7.1. Finally, Wurmser and Filitti-Wurmser (24) in France and Stotz, Sidwell and Hogness (25) in Chicago, measured the potential of pure cytochrome $c$ isolated from heart muscle. The former measured the equilibrium potential in mixtures of reduced and oxidized cytochrome $c$, the proportion being determined spectrophotometrically. They obtained the value $\mathrm{E}_{\mathrm{o}}^{\prime}=$ +0.254 v. between $\mathrm{pH} 5.0$ and 8.0. Stotz et al. used a purely spectrophotometric method and obtained a value of $+0.262 \mathrm{v}$. in the same $\mathrm{pH}$ range. The spectrophotometric method consisted in measuring accurately the amounts of oxidized and reduced indicator and cytochrome in equilibrium with each other. The potential of the indicator being known, the potential of the cytochrome could be readily calculated. The results recorded by the two groups were reached independently and represent good agreement. They are both in essential agreement with the potential of $+0.27 \mathrm{v}$. reported by Ball (26), who was able to estimate the potentials of the three cytochromes as they existed in a heart muscle extract. At a physiological $\mathrm{pH}$, therefore, cytochrome $c$ has about the same potential as the hydroquinone-quinone system.

Cytochrome b.-In the reduced state this cytochrome component possesses an alpha-absorption band at $5640 \mathrm{~A}$. and a beta-band at $5300 \mathrm{~A}$. It appears to be more closely bound to the insoluble material in tissue extracts than is cytochrome $c$. Nevertheless Yakushiji and Mori (27) claim to have isolated cytochrome $b$ in a soluble form. It seems doubtful from their method of preparation whether the product obtained could be an undenatured cytochrome $b$. In some of the original extracts the reduced band is not at $5640 \mathrm{~A}$., but as purification proceeds this band is shifted to the normal position of reduced cytochrome $b$. They believe that the hemin portion of their product is ordinary protohemin. Since several other proteins combined with protohemin to form spectroscopically and catalytically similar hemochromogens, it is difficult to believe that these workers actually obtained cytochrome $b$.

Judged from its behavior in tissue extracts, cytochrome $b$ appears to be a thermolabile hemin-protein complex. Unlike cytochrome $c$, the $b$ component is autoxidizable. Since this component reaches equilibrium with other reversible systems in a heart muscle extract, Ball (26) was able to estimate its potential as $-0.04 \mathrm{v}$., the lowest of the cytochrome components. It does not combine with carbon monoxide or other respiratory inhibitors (10). 
Cytochrome a.-Cytochrome $a$ is another component whose properties can be judged only in a crude tissue extract. It was originally designated as the component which in the reduced state possessed an alpha-band at 6000-6050 A. Upon more careful analysis, Ball (26) and Keilin and Hartree (10) discovered that this band was not homogeneous to reduction or to various reagents; that is, the absorption in this region must be attributed to more than one substance. Their results might be interpreted to mean that the portion of the band nearer $6050 \mathrm{~A}$. is to be attributed to cytochrome $a$. Because of this complication, the properties of cytochrome $a$ have not been definitely established. Keilin and Hartree (10) conclude that it does not combine with carbon monoxide or cyanide. It is reduced by the same agents as the other cytochromes. Its potential has been estimated by Ball (26) as $+0.29 \mathrm{v}$, a value which is perhaps less certain than those of the other cytochromes.

Very recently Yakusizi and Okunuki (28) claim to have isolated cytochrome $a$ from heart muscle. The muscle pulp was extracted with sodium cholate and alkaline phosphate. Ammonium sulfate fractionations followed by redissolving in the cholate mixture yielded a product which was, in the oxidized state, of a red-brown color. When it was reduced it was green, indicating that the prosthetic group was of the "mixed" or Spirographis hemin type. The reduced compound showed a strong absorption at $6050 \mathrm{~A}$. and a weak band at 5130 A., carbon monoxide having no effect on the spectrum. Reduced cytochrome $c$ was partially oxidized by the oxidized form of this compound. The latter properties are in agreement with our concept of cytochrome $a$, and this important finding should be confirmed and extended.

There is some evidence that the potentials of the yeast cytochromes differ from those of the heart cytochromes. But the only measurement on a pure component has been with heart cytochrome $c$. It will be recalled that Green (23) obtained a value of $\mathrm{E}_{\mathrm{o}}{ }^{\prime}=+.127 \mathrm{v}$. for yeast cytochrome $c$, and the recent work of Baumberger (29) is in agreement with this finding. The latter work, however, requires certain comments. Baumberger was able to measure simultaneously the light absorption at various wave lengths (photoelectrically) and the $\mathrm{E}_{\mathrm{h}}$ levels of a yeast suspension. The suspension was vigorously stirred by oxygen-nitrogen mixtures, which likewise eventually established a constant $E_{\mathrm{h}}$ level. By varying the gas mixture and hence the $\mathrm{E}_{\mathrm{h}}$ level, the presence or absence of the cytochrome bands could be determined by changing the wave 
length of the incident light and observing the galvanometer deflections of the photoelectric device. In this way he arrived at the above potential for cytochrome $c$.

Perhaps more startling was his finding that all three cytochromes appeared to have the same potential; that is, at a given $\mathrm{E}_{\mathrm{h}}$ level all were equally reduced. This is distinctly a contradiction of Ball's finding that in a heart muscle extract the cytochrome potentials differed markedly. In Ball's work, however, the relative degree of reduction of the cytochromes was measured when in equilibrium with systems of known potential and systems known to react with the cytochromes, whereas in Baumberger's work the normal reductants within a more organized structure establish the equilibrium. A legitimate question concerning these experiments would be whether it can be assumed that the potential recorded by a platinum electrode in a yeast suspension is the same as that existing within the cell. And, furthermore, is it not likely that different points in the organized cells actually have very different potentials? The data might indeed be taken as evidence for the latter hypothesis. Baumberger suggests the possibility of a molecular aggregate of the three cytochromes which is oxidized or reduced as a whole or in which the three cytochromes do have the same potential.

In a more disorganized structure, such as a heart muscle extract, these relations apparently do not exist, and it is not unlikely that this explains the great difference between the frequency of oxidation and reduction of cytochromes in the intact yeast as compared with that in extracts. Certain it is that in the study of tissue respiration the problem of adsorption and dependence of function on organized structure is met most frequently in the consideration of the cytochrome system (see "Oxidation and Cell Structure" in Korr, 30).

Cytochrome Oxidase (Cytochrome $a_{3}$ ).-It may be recalled that in 1924 Warburg (31), upon observing the cyanide sensitivity of cellular respiration in conjunction with the catalytic behavior of the hemin-charcoal model, gave the name Atmungsferment to the catalytically active iron compounds involved in cellular respiration. This study continued with measurements of the inhibition of yeast respiration by carbon monoxide and its reversibility by light $(32,33)$. By measuring this effect at various light frequencies, the relative carbon monoxide spectrum and later the absolute carbon monoxide spectrum of the Atmungsferment were determined $(34,35)$. It was renamed the Sauerstoffubertragendes Ferment or oxygen- 


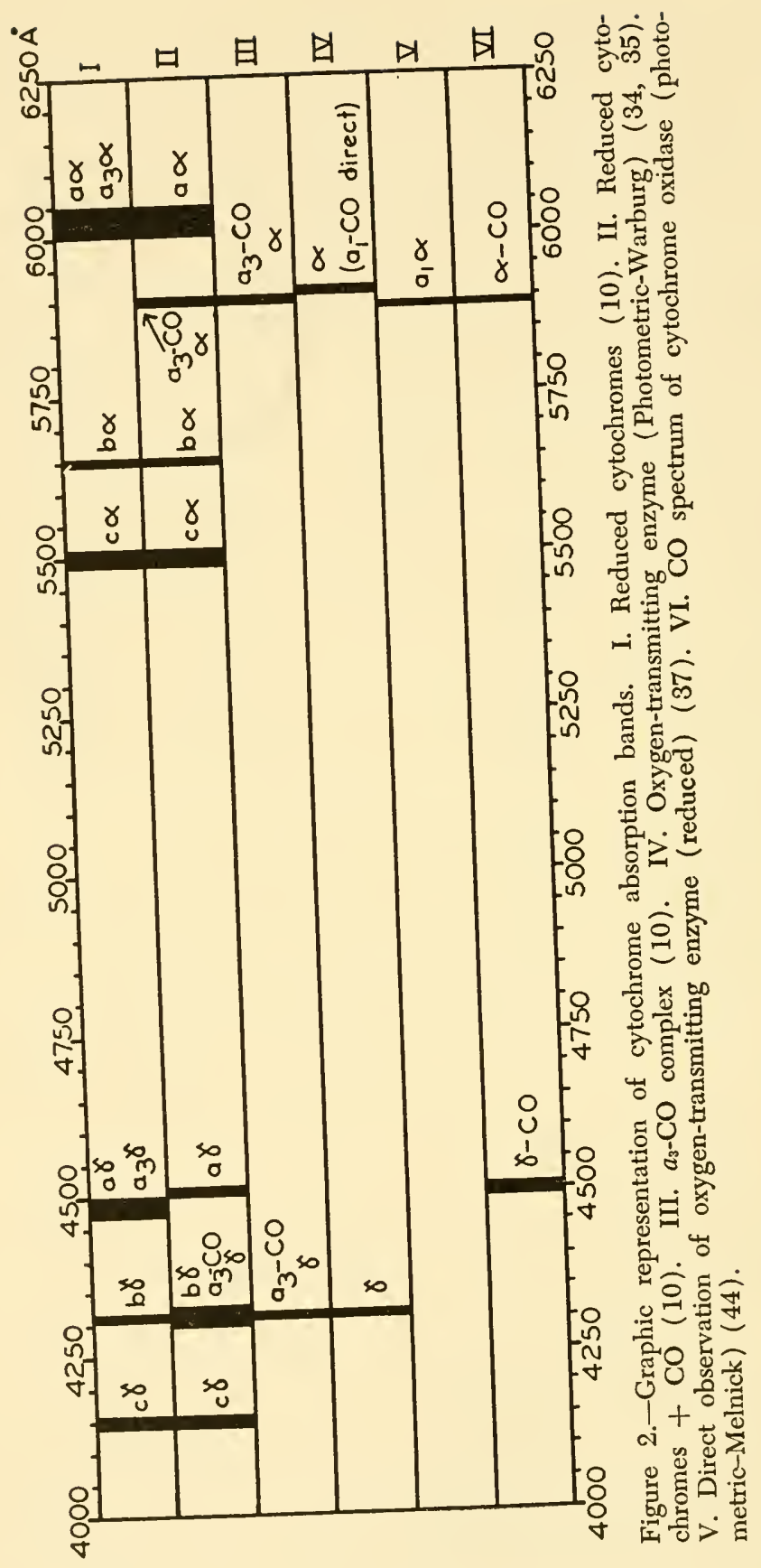


transferring enzyme. The spectrum was obviously that of a hemincontaining compound which resembled in type that of Spirographis hemin (36). The alpha-band of the reduced carbon monoxide complex lies at $5920 \mathrm{~A}$. and the gamma-band at $4320 \mathrm{~A}$. Finally, in the highly respiring Bacterium Pasteurianum (Acetobacter pasteurianum), under anaerobic conditions, a weak band was observed at 5890 A. which was attributed by Warburg and Negelein (37) to the reduced form of the oxygen-transferring enzyme itself, since carbon monoxide shifted the band to $5920 \mathrm{~A}$. On the other hand, cyanide produced a band at $6390 \mathrm{~A}$. which, since it may be observed even in the simultaneous presence of the $5890 \mathrm{~A}$. band, need not be a derivative of the oxygen-transferring enzyme. Keilin (38) believes, however, that the $5890 \mathrm{~A}$. band is only a degradation product of cytochrome $a$ and is seen only in certain bacteria. In fact, Fujita and Kodama (39) observed the $5890 \mathrm{~A}$. band in bacteria only when the cytochrome $a$ band was absent and have named this band cytochrome $a_{1}$. Certainly the best criteria by which to establish the identity of a compound with Warburg's oxygen-transferring enzyme would be the positions of the carbon monoxide absorption bands (5920 and 5320 A.).

Keilin's work (40) with "indophenol oxidase" pointed to the identity of this enzyme with the Warburg enzyme. The oxidase brought about the aerobic oxidation of the cytochromes, was inhibited by cyanide, and showed a light-reversible inhibition with carbon monoxide.

Because of the similarity it has been generally believed that Warburg's "oxygen-transmitting enzyme" and Keilin's presently-called cytochrome oxidase are identical. Until recently, however, Keilin had not observed a band that he could attribute to the carbon monoxide complex of the oxidase.

In 1939 Keilin and Hartree (10) believed that they had identified spectroscopically in heart muscle extracts a new cytochrome, $a_{3}$, which might be identical with the oxidase. They concluded that the cytochrome $a$ band at $6000-6050 \mathrm{~A}$. is actually due to components $a$ and $a_{3}$, since upon addition of carbon monoxide this band divides and a new one appears at $5900 \mathrm{~A}$. With the aid of strong cane sugar or glycerine solutions, or bile salts, to clarify the solutions for spectroscopic examination, they were able to examine further the Soret or gamma-bands of the cytochromes. Simultaneously with the above shift, a portion of the $4480 \mathrm{~A}$. band is shifted to $4320 \mathrm{~A}$. These two new bands represent the carbon monoxide complex of 
the new component, $a_{3}$. Since these positions correspond to those of the Warburg enzyme, $a_{3}$ may be identical with this enzyme. On the other hand, the alpha-band of the compound itself is claimed to be at $6000 \mathrm{~A}$., whereas Warburg and co-workers $(41,42,43)$ believe their compound to have a band at $5890 \mathrm{~A}$. This compound (also called cytochrome $a_{1}$ ) likewise reacts with oxygen, and formation of its cyanide compound prevents reoxidation of the other cytochromes. Furthermore, its carbon monoxide compound has an alpha-band at 5920 A.

Cytochrome $a_{3}$, in either the reduced or the oxidized form, combines with potassium cyanide. The cyanide complex of the reduced form is readily autoxidizable, whereas that of the ferric form is not easily reduced. Ferric cytochrome $a_{3}$ also reacts with hydrogen sulfide, sodium azide, and hydroxylamine. It is thermolabile and easily destroyed by organic solvents, acids, or alkalies. Cytochrome $a_{3}$ is reduced along with the other cytochromes by e.g., succinate. It is also autoxidizable. It is therefore believed that $a_{3}$ is also identical with cytochrome oxidase.

The authors have themselves offered certain objections to the above conclusion:

1. It was impossible to demonstrate the reduction of cytochrome $a_{3}$ by added reduced cytochrome $c$, but technically these experiments were not satisfactory. In this connection it is interesting that Ball (26) noticed that the portion of the 6000-6050 A. band attributed to $a_{3}$ by Keilin has a higher potential than any of the other cytochromes, an expected but not an essential condition for oxidase function.

2. The carbon monoxide compound of ferro-cytochrome $a_{3}$ did not appear to be sensitive to light anaerobically. The effect of light, however, may become apparent only in the presence of oxygen, which oxidizes the $a_{3}$ component and thereby prevents its reaction with carbon monoxide. Such an explanation of the light effect could also explain its property of relieving inhibition of carbon monoxide.

3. Finally, it has been found possible in the presence of carbon monoxide to oxidize cytochromes $a, b$, and $c$ by air while the spectrum of the carbon monoxide complex of reduced $a_{3}$ remains visible. It is therefore difficult to explain the oxidation of $a, b$, and $c$ through the $a_{3}$ component.

In general, it may be said that the Keilin and Hartree paper by no means clarifies the whole problem of the identity of cytochrome oxidase and oxygen-transferring enzyme or of either one with the 
new $a_{3}$ component. The situation is understandable, however, when one considers the number of hematin compounds which exist in tissue preparations and the fact that apparently the same component may vary slightly in the position of its absorption band in different biological materials.

An experiment that has been much needed has finally been published as a short note by Melnick (44), namely, the photochemical determination of the carbon monoxide spectrum of cytochrome oxidase. This was accomplished by employing a phosphate extract of heart muscle with succinate as substrate. On the assumption that the oxidase is the only functional substance present in the preparation which forms a light-dissociable carbon monoxide complex, the spectrum measured should be, by Keilin's own definition, cytochrome oxidase. The spectrum obtained was that of a pheohemin compound. The alpha-band was located at $5890 \mathrm{~A}$., which agrees very well with that of the oxygen-transferring enzyme in yeast and bacteria, as well as with the carbon monoxide complex of cytochrome $a_{3}$ seen directly. But in the case of the gamma- or Soretband, Melnick finds a band at $4500 \mathrm{~A}$, which does not agree with the carbon monoxide band of the oxygen-transmitting enzyme (Warburg) nor with that of $a_{3}$ (Keilin). The whole situation therefore remains clouded and awaits chemical separation and identification for its clarification.

Keilin and Hartree (10) believe that because of the association of cytochromes $a$ and $a_{3}$ the two components are intimately related. They may have an identical heme nucleus, since on alkali denaturation and addition of pyridine they yield the same hemochromogen. They are both sensitive to heat, alcohol, acetone, and extreme changes in $\mathrm{pH}$.

It has been suggested that cytochrome oxidase may be a copper protein. The evidence is quite indirect, such as the wide distribution of copper, the ability of copper salts to oxidize cytochrome $c$, and certain similarities between cytochrome oxidase and the coppercontaining polyphenol oxidase $(45,46)$. It may be pointed out that copper does appear to be essential in the formation of cytochrome oxidase. Cohen and Elvehjem (47) have found it to be essential for the regeneration of cytochrome $a$ and oxidase in anemic rats, and Yoshikawa (48) finds it to be a stimulus to the oxidase activity of yeast cultures. Most conclusive is the recent work of Schultze (49, 50 ), who showed that copper was necessary for the maintenance and formation of cytochrome oxidase in rat liver and heart, and that 
the regeneration of cytochrome oxidase in the bone marrow of anemic rats was extremely rapid following administration of copper. Important as these findings are, they do not constitute proof that cytochrome oxidase contains the copper any more than similar findings with hemoglobin regeneration.

Graubard (51) claims actually to have isolated a water-soluble cytochrome oxidase from uterus. It is very labile and its action is inhibited by copper inhibitors. He therefore claims that at least this oxidase contains copper as the active metal. Certainly any claims for the identity of a water-soluble or copper-containing oxidase with cytochrome oxidase must be supported by many experiments giving evidence that the material conforms with the existing definition of cytochrome oxidase.

The work of Altschul, Abrams, and Hogness $(52,53)$ in connection with the oxidase is of particular interest. They first reported the isolation from yeast of a soluble cytochrome oxidase which aerobically oxidized cytochrome $c$, and which was inhibited by cyanide and carbon monoxide. Upon concentration, it was noted, the activity of the enzyme was inhibited by catalase. It was then found that during reduction of the substrate, cytochrome $c$, hydrogen peroxide was produced as a contaminant and oxidation was actually a catalyzed oxidation by peroxide. This enzyme, being specific, is now called cytochrome $c$ peroxidase. Because so little is known about the physiological mechanism of the aerobic oxidation of the cytochromes and of the function of hydrogen peroxide, this very active enzyme may, be of no small importance physiologically.

From the standpoint of isolation, therefore, we still have only cytochrome $c$. The other cytochromes and the oxidase are still associated with insoluble particles and have resisted separation. The usual opalescent alkaline phosphate extract of heart muscle, which contains these substances, has been the subject of a physico-chemical investigation by Stern (54). Observations of this material in the ultracentrifuge and electrophoresis reveal properties similar to those of other macro-molecular materials, such as fractions from Rous chicken sarcomata. Such a dispersed suspension of particles contains lipids, nucleic acid, hemin, and other constituents. Stern feels that it is largely a matter of definition whether such a "monodispersed" suspension should be called a mechanical dispersion or a true solution, and he predicts that when the individual components are isolated they will no longer display their characteristic biological orientation. This view does not and should not discourage attempts 
to isolate the individual components. Although such heart muscle preparations have thus far resisted fractionation, one factor, the diaphorase or coenzyme factor (a flavoprotein) has been separated from such a preparation (55).

Euler and Hellström (56) claim to have effected a separation of the cytochromes by an ammonium sulfate fractionation of a sodium cholate clarified preparation. The first fraction to precipitate contained cytochromes $a$ and $b$, the second only cytochrome $b$, and the third, cytochrome $c$. The activity of some of these fractions toward succinate does not, however, bear out the claim for any extensive fractionation (see Keilin and Hartree, 9). Such precipitates simply resuspend in buffers to yield the usual opalescent preparations. Nevertheless the clarifying or so-called peptizing action of the bile salts is interesting. I have found (8) in a few experiments that sodium desoxycholate at a neutral $\mathrm{pH}$ yields a virtually clear solution of the original turbid oxidase preparation. Fractional salt precipitations have not, however, yielded any striking results. In view of the action of bile salts with lipids, it is possible that the difficulties of separating the cytochrome components may lie in their association with or their presence as lipo-proteins.

Reduction of the Cytochromes by Other Respiratory Systems

The fundamental position of the cytochromes in cellular respiration is emphasized by the fact that tissue respiration is so completely blocked by cyanide (57), and by the experiment of Haas (58) in which it was demonstrated that the rate of alternate oxidation and reduction of cytochrome $c$ in intact yeast cells could account for all the oxygen consumption of the yeast.

The succinate-succinic dehydrogenase system has long been recognized as a reducing system for the cytochromes, and this connects the important Szent-Györgyi-Krebs cycle with the cytochromes. On the other hand, the details of this reduction are by no means clear. Thus in 1939 Hopkins, Lutwak-Mann, and Morgan (59) prepared a succinic dehydrogenase from heart muscle which with succinate did not reduce cytochrome $c$, but did nevertheless reduce methylene blue. The preparation was made in such a way (with alcohol treatments) that no cytochrome oxidase activity remained. Their result suggests another intermediate between succinic dehydrogenase and cytochrome $c$.

Stern and Melnick $(54,60)$ in their ultracentrifuge studies found that the sedimented material showed typical succinic dehydrogenase 
activity toward methylene blue and oxidase activity toward $p$-phenylenediamine or hydroquinone. It lacked, however, the capacity to oxidize succinate, apparently being unable to reduce cytochrome $c$. When supernatant fluid from the ultracentrifuge run was added, aerobic activity of the preparation toward succinate was restored. The unknown material is evidently a substance of lower molecular weight (estimated at 140,000), is heat-labile, and is removed by trichloracetic acid, hence is probably a protein. It cannot be identified with aluminum (61), catalase, or the Straub flavoprotein (55).

Recently Keilin and Hartree (9) have tested and analyzed the effects of several factors on succinate and $p$-phenylenediamine oxidation by typical heart muscle extracts. Among their findings were these: 1. Narcotics inhibited the oxidation of succinate by the cytochrome system more strongly than the oxidation of methylene blue. Since not only reduction of the cytochrome components was inhibited, but also oxidation of cytochrome $b$, it is possible that these findings are related to the function of cytochrome $b$ in succinate oxidation. 2. Preparations treated with alcohol modified irreversibly the spectrum of cytochromes $a_{3}, a$, and $b$ and destroyed oxidase activity. Such preparations did not reduce cytochrome $c$, although they retained their ability to reduce methylene blue. 3 . Treatment with acetic acid ( $\mathrm{pH} 5.0$ for one hour) did not affect $p$-phenylenediamine oxidation, but destroyed the ability of the succinate system to reduce cytochrome $c$. Again, methylene blue reduction was still possible. Spectroscopically, the absorption bands of the cytochromes were normal, except that cytochrome $b$ appeared to be no longer autoxidizable. Evidently cytochrome $b$ had undergone some change. 4. Treatment with pancreatin gave a preparation similar to the acid-treated preparation.

The results suggest that cytochrome $b$ may be the labile link between succinic dehydrogenase and cytochrome $c$, although the possibility that a flavin or another hematin is a link is by no means excluded. It may be recalled that the potential of cytochrome $b$ $(-0.04$ v. $)$ places it in a favorable position as such a link. Keilin and Hartree have suggested as an alternative the possibility that the failure to react with cytochrome $c$ "may be due to an irreversible change in the colloidal structure of the preparation accompanied by a loss of accessibility of the succinic system to $c$, which is a nondiffusible protein while it remains still accesible to small and diffusible molecules of methylene blue." 
It was long desirable to find a link between the di- and triphosphopyridine nucleotides and the cytochrome system. For some time it has been known that the old yellow enzyme, i.e., the Warburg-Christian flavoprotein (62), was readily reduced by triphosphopyridine nucleotide (63), but only very slowly oxidized by cytochrome $c$ (64). This link has recently been established by the excellent isolation work of Haas, Horecker, and Hogness (65), who isolated a flavo (mononucleotide)-protein which rapidly reduces cytochrome $c$. It has been given the functional name "cytochrome reductase." The coenzyme II dependent systems have thus been satisfactorily linked to the iron-containing system. Cytochrome $c$ reductase does not link reduced coenzyme I with cytochrome $c$ (66). Haas, Horecker, and Hogness (65) believe that since the reductase loses its power to reduce cytochrome $c$ when subjected to chemical treatments common to the preparation of the old WarburgChristian flavoprotein, it is very probable that the latter represents a denatured product of cytochrome reductase.

The story is less satisfactory than in the case of coenzyme I. In 1937 Adler, Euler, and Hellström (67) discovered an enzyme which they called "diaphorase," and independently Green and Dewan (68) investigated what was apparently the same enzyme, which they called "coenzyme factor." This factor appeared to link coenzyme I with the cytochrome system. Of significance for the present discussion is the fact that Green and Dewan (68) stated that cytochromes $a$ and $b$ but not $c$ were involved in the reaction. The absence of cytochrome $c$ in their preparation has been challenged by Hawthorne and Harrison (69) and by Lockhart and Potter (66). The latter authors have in fact demonstrated that cytochrome $c$ is a link in the aerobic oxidation of coenzyme I. They also have used two types of "diaphorase" preparation, one of which catalyzed the coenzyme I reduction of cytochrome $c$ and the other did not. Both, however, could catalyze the reduction of methylene blue. This recalls the findings in connection with succinate reduction of cytochrome $c$; in fact, Lockhart and Potter have noted that the preparation unable to reduce cytochrome $c$ contained no cytochrome $b$. Thus comes the suggestion but not the proof that cytochrome $b$ may be involved as a link between coenzyme I dependent systems and cytochrome $c$.

Straub (55) has isolated from heart muscle a flavoprotein which is considered to be identical with the coenzyme factor or diaphorase. Corran, Green, and Straub (70), in studying the catalytic proporties of this flavoprotein, find that its reduced form is only slowly autoxi- 
dizable and that it does not react with cytochrome $c$ but only with "carriers" such as methylene blue. It is not yet known whether this protein requires another link (such as cytochrome $b$ ) for cytochrome $c$ reduction, or whether it is actually a denatured product which has lost its ability to reduce cytochrome $c$, analogous to the old Warburg-Christian yellow enzyme and the newer cytochrome reductase.

\section{Catalytic Relations of the Cytochromes and Oxidase}

"Indophenol oxidase" was long recognized as the substance in tissues which produced the aerobic oxidation of Nadi reagent or $p$-phenylenediamine (3). This name was retained in spite of the finding of Keilin (71) that the addition of cytochrome $c$ accelerated the oxidation of cysteine by "indophenol oxidase" and a similar finding by Stotz, Harrer, Schultze, and King (72) with respect to ascorbic acid. When pure cytochrome $c$ became available, it was not difficult to study the relation of oxidase and cytochrome $c$ in the oxidation of various substrates. The high oxidation-reduction potential of cytochrome $c$ that had been noted suggested that the action of indophenol oxidase was due to an unspecific reduction of the cytochrome $c$ which it contained, followed by an aerobic catalyzed oxidation of the reduced cytochrome. Keilin and Hartree (73), upon noting the accelerating effect of cytochrome $c$ on the oxidation of several substrates, renamed the oxidase "cytochrome oxidase." Stotz, Sidwell, and Hogness (74) had come to the same conclusion and had prepared an oxidase which was largely free of cytochromes

Table 1.-The sensitivity of hydroquinone and $p$-phenylenediamine oxidations to cyanide*

(Reference 74)

\begin{tabular}{ccc}
\hline \hline & \multicolumn{2}{c}{ Percentage of inhibition } \\
\cline { 2 - 3 } $\begin{array}{c}\text { Cyanide concentration } \\
\text { mM } \times 10^{6} \text { total }\end{array}$ & $\begin{array}{c}\text { Hydroquinone } \\
\text { oxidation }\end{array}$ & $\begin{array}{c}p \text {-phenylenediamine } \\
\text { oxidation }\end{array}$ \\
\hline 0 & 0 & 0 \\
20 & 57 & 52 \\
40 & 82 & 70 \\
60 & 92 & 77 \\
100 & 99 & 82 \\
120 & 100 & 83 \\
240 & 100 & 85 \\
\hline
\end{tabular}

${ }^{*} \mathrm{~T}=38^{\circ}$ C.; pH, $7.15 ; 23.5 \times 10^{-6} \mathrm{mM}$ cytochrome $c$ total. 
$c$ and $b$. This preparation was essentially unable to oxidize either hydroquinone or $p$-phenylenediamine without the addition of cytochrome $c$.

A study of the cyanide sensitivity of hydroquinone and $p$-phenylenediamine oxidation (see Table 1) suggested, because of the redox potential relations of the cytochromes and the substrates, that hydroquinone oxidation involved only the oxidase and cytochrome $c$, whereas $p$-phenylenediamine could be independently oxidized by cytochrome $b$ as well. The cyanide-resistant portion of $p$-phenylenediamine oxidation is probably due to the autoxidizable, cyanide-resistant cytochrome $b$.

The oxidation of hydroquinone is a function of both the oxidase and cytochrome $c$; hence its oxidation by tissue extracts is not an absolute method for determining either substance. The effect of cytochrome $c$ in accelerating the rate of hydroquinone oxidation by a heart muscle oxidase preparation is shown in Figure 3.

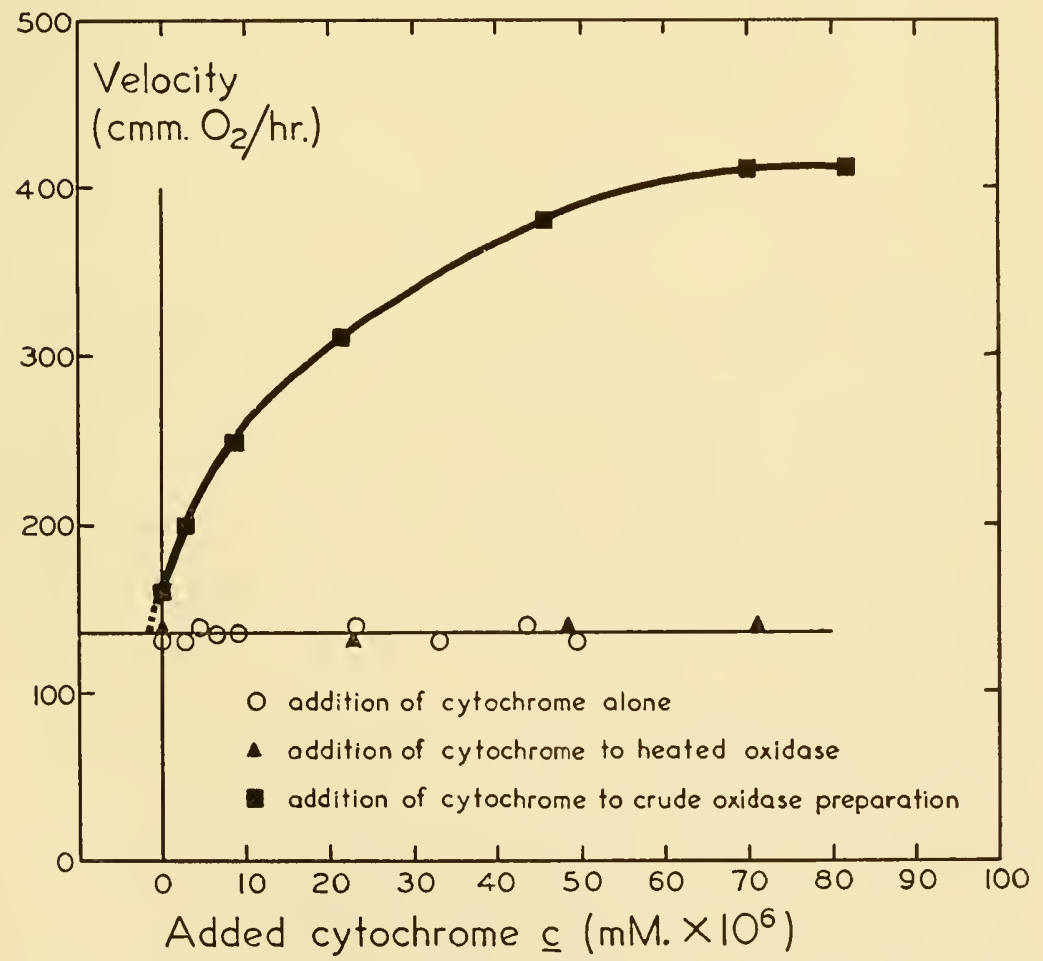

Figure 3.-The oxidation of hydroquinone by the oxidase-cytochrome $c$ system. $\mathrm{T}=38^{\circ} \mathrm{C}$., $\mathrm{pH} 7.15$, hydroquinone $0.033 \mathrm{mM}$. total. 
The reduction of cytochrome $c$ by hydroquinone is very rapid; hence the rate-controlling reaction here is the oxidation of the reduced cytochrome. Several curves such as those in Figure 3 have been found to comply with the laws of a typical enzyme-substrate complex (75), indicating that the oxidase and cytochrome $c$ form such a complex. A study of such curves in relation to the effect of cyanide and carbon monoxide led to the conclusion that the action of these inhibitors on the reaction was concerned with the oxidase component (75).

On the other hand, in the presence of an excess of cytochrome $c$ the velocity of oxidation of hydroquinone (correcting for autoxidation) was directly proportional to the amount of oxidase added. This offers a method of estimating, in arbitrary units, the cytochrome oxidase activity of tissues.

From hydroquinone and $p$-phenylenediamine tests it appears that successive acetic acid precipitations remove the larger part of the cytochrome $c$ and some of the cytochrome $b$. By two precipitations with acetic acid and a long dialysis an active oxidase preparation can be obtained which shows very little cytochrome $c$ or $b$, although the oxidase activity is likewise greatly diminished.

\section{Determination and Distribution of Cytochrome c and}

\section{CyTOCHROME OXIDASE}

Junowicz-Kochalaty and Hogness (76) have developed a method for estimating cytochrome $c$ in tissues. Relatively large amounts (100 grams of tissue) are worked up through the initial steps of Keilin's isolation procedure (5) to the point where traces of hemoglobin and myoglobin are the principal colored impurities. The cytochrome $c$ is then measured spectrophotometrically. The use of measurements at three wave lengths permits of calculations to correct for the hemoglobin and myoglobin. These authors found pigeon breast muscle and beef heart muscle high in cytochrome $c$, tumor tissue very low.

Stotz (77) has developed a method for determining cytochrome $c$ in rat tissues. The ground tissue is extracted with trichloracetic acid, the extract neutralized to eliminate further inactive protein, and the cytochrome precipitated by phosphotungstic acid. After solution in dilute ammonia, the phosphotungstate is eliminated with barium. The final solutions are tested manometrically for their power to accelerate oxidation of hydroquinone by a heart muscle oxidase preparation. A calibration curve must be prepared, pure cytochrome $c$ being used. 
In applying this test to various rat tissues it was found that agreement between animals was reasonably good; the order of activity of the tissues was the same in all the rats studied. The average results of the cytochrome $c$ estimations in the tissues of ten rats are recorded in Table 2.

Table 2.-Comparison of cytochrome oxidase and cytochrome $c$ activities of rat tissues

(Reference 77)

\begin{tabular}{|c|c|c|c|c|c|c|c|c|c|c|c|c|c|c|c|}
\hline \multicolumn{9}{|c|}{ Oxidase } & \multicolumn{7}{|c|}{ Cytochrome $c$} \\
\hline & & & & & & & & $\begin{array}{l}\text { Units per } \\
\text { mg. dry } \\
\text { tissue }\end{array}$ & & & & & & & $\begin{array}{l}\text { mg. per g. } \\
\text { dry tissue }\end{array}$ \\
\hline Heart & & & & & & & & . 9.7 & Heart & . & . &. & . & . & . 2.34 \\
\hline Kidney & 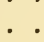 & & 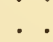 & . & & & 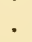 & . 4.7 & Kidney . . & . . . & . . & .. & . & . & . 1.36 \\
\hline Brain & & & 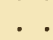 & & & & & . 3.5 & Skeletal muscle & e. & . & . & . & & . 0.68 \\
\hline Skeletal & $\mathrm{lm}$ & uscle & 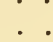 & & & & & $\begin{array}{l}\text {. } \quad 2.3 \\
\end{array}$ & Brain ... & . . & . & . & . & & . 0.35 \\
\hline Liver & . . & . . . & t & & & & $\cdot$ & . 1.7 & Liver . . & . . & . & . & . & 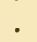 & . 0.24 \\
\hline Spleen & . & & 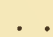 & . & & . & . & . $\quad 1.6$ & Spleen . . & $\cdots$ & . & . & . & . & 0.21 \\
\hline Lung . & . & & 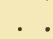 & . & & . & . & . $\quad 1.3$ & Lung. . . . & . . & . & . & . & 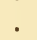 & . 0.14 \\
\hline Test is & . & & & & & . & . & . 1.1 & Embryo (early) & ) . & . & . & . & . & . 0.03 \\
\hline Diaphra & agm & mus & cle. & & & . & • & . $\quad 0.72$ & Embryo (late) & . . & . . & - . & . & 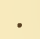 & 0.18 \\
\hline Large in & ntes & stine & & & & & & 0.36 & Tumor R-256 & . . & . . & . . & . & 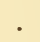 & . 0.02 \\
\hline Embryo & o (es & arly, & late) & & & & & . 1.1 & Tumor R-39 & . . & . & .. & . & & . 0.03 \\
\hline Tumor & $\mathrm{R}-2$ & 256. & , & & & & & . $\quad 2.9$ & Tumor spontan & neous & & . & . & . & . 0.01 \\
\hline Tumor & spo & intane & eous & & & • & • & . $\quad 2.4$ & & & & & & & \\
\hline
\end{tabular}

Most striking is the low cytochrome $c$ content of embryos and the tumors studied. It may be noted that the cytochrome $c$ content of the embryos increases just before birth. With the present knowledge of the importance of cytochrome $c$ in respiration, the low $c$ content of these tissues may be at least one factor responsible for the aerobic glycolysis of these tissues.

Potter and Dubois (78) have likewise developed a micro method for estimating cytochrome $c$, the actual determination being made with a photoelectric spectrophotometer; the light absorption is measured before and after specific enzymatic reduction with succinate. The values they obtained with rat tissues are in remarkably good agreement with those recorded by Stotz. They have found a low $c$ content in several tumors thus far studied.

The manometric estimation of oxidase activity in rat tissues may be carried out on ground dialyzed tissue in the presence of excess cytochrome with hydroquinone as a substrate (77). Schultze (49) uses in addition semicarbazide, which maintains a constant rate of oxidation over a longer period, a helpful modification. 
The distribution of oxidase in rat tissues is also illustrated in Table 2. Perhaps the most interesting thing about these data is the fact that the oxidase activity of the tissues parallels quite closely their cytochrome $c$ content, although in the case of the tumors and embryonic tissue there is no lack of cytochrome oxidase. This parallelism may indicate a close chemical similarity between, or common origin of, cytochrome $c$ and cytochrome oxidase.

A decrease in oxidase activity has been noted in various tissues of the rat in anemia $(49,50)$ and in guinea pig tissues during acute scurvy (79).

\section{Physiological Functioning of the Cytochrome System}

Flexner and Stiehler $(80,81)$ have made some very interesting observations on the funct. nal development of the cytochrome system. By histochemical methods they studied the changes in the chorioid plexus of the fetal rig, especially during the phase when the spinal fluid changes from an ultra-filtrate to a secretion. Oxidase activity was measured by blue-staining with dimethyl $p$-phenylenediamine and alpha-naphthol, a test which of course measures the combined oxidase and cytochrome activity. Oxidation-reduction potentials were estimated by introduction of oxidation-reduction indicators intravascularly or supravitally. It was found that previous to the secretory phase the concentration of "indophenol oxidase" was the same in the epithelium and stroma and that there was no potential difference between the two. In the secretory phase, however, the oxidase was concentrated in the epithelium and a potential difference developed, more positive in the epithelium. It was concluded that the functional changes occurring with the onset of secretion are correlated with the potential difference set up as a result of the selective developisent of the oxidase (cytochrome) system in the epithelium. The selective transference of dyes across the secretory plexus was abolished by cyanide, and this was associated with a loss of the potential difference between epithelium and stroma.

Flexner, Flexner, and Straus (82) have likewise studied the cytochrome system in the cerebral cortex of the fetal pig. During the first half of gestation $p$-phenylenediamine was not actively oxidized, but during the second half an active oxidation system was present. Employing the test for cytochrome oxidase (using excess cytochrome $c$ ), they found that there was no lack of oxidase in any phase of gestation and hence the change in $p$-phenylenediamine 
oxidation was due primarily to development of cytochrome $c$. Nevertheless the $\mathrm{Q}_{2}$ of the tissue increased little during this period and at all times was over 90 per cent sensitive to $0.001 \mathrm{M}$. cyanide. Thus there is an apparent anomaly in that cyanide, the inhibitory action of which is generally considered to be on the cytochrome oxidase system, is inhibiting a respiratory system which does not even contain a complete cytochrome system. This is not the first instance in which the "single point of attack theory" of cyanide has been questioned. Parallel experiments with cyanide and azide (see Stannard, 83) on intact tissues have also suggested that cyanide probably combines with one or more other enzymes involved in respiration. The question arises, therefore, what is the nature of the "oxygen-activating" system in the absence of cytochrome c? If cyanide can also block an enzyme systeri near the "dehydrogenase end" of respiration, then flavin enzymes as well as cytochrome $b$ (which is relatively cyanide-stable and autoxidizable) become possibilities for this role, despite the fact that their oxidation is generally considered to be through the oxidase system.

A somewhat similar situation arises in Stannard's experiments (83) on the "resting" and "activity" oxygen consumption of frog muscle. It was found that the resting metabolism was insensitive to azide, although sensitive to cyanide. On the other hand, the activity metabolism (caffeine or electrical stimulation) was greatly inhibited by azide. When the azide concentration in the stimulated preparation was increased, the inhibition 'came to an abrupt stop, leaving a respiration equal to that of the resting state. The latter finding excludes the possibility that the azide stability of the resting respiration can be explained by failure of the inhibitor to penetrate or its absence in a form capable of exerting its typical inhibition on the oxidase system. Stannard $(84,85)$, who has studied the anaerobic glycolysis of frog muscle, does not believe that the azide insensitivity of resting muscle can be explained by the lack of substrate for "saturation" of the oxidizing enzymes. He has concluded that the oxygen transfer for the resting metabolism and for the extra metabolism resulting from stimulation are due to different systems. The extra metabolism due to stimulation was apparently by way of the cytochrome system, but the nature of the "oxygen-activating" enzymes of the resting metabolism remain unknown.

Recently Korr (30) has carried out experiments on the metabolism of slices of mammalian tissues during rest and during a stimulated phase. Salivary glands stimulated by acetylcholine or adrenalin, 
pancreas by secretin, and myometrium by oxytocin all show that large increases of respiration attend the change from a state of rest to one of activity. The finding of azide-stable and relatively cyanide-stable respiration, combined with spectroscopic observation of the cytochrome bands, indicated that the resting metabolism was not proceeding through the cytochrome system, but that the extra metabolism that followed stimulation was mediated through this system. It is important to note that in the resting state the cytochrome system was nevertheless "available" for the oxidation of $p$-phenylenediamine. It was postulated that the cytochrome system becomes "geared" or "linked" to the substrate-dehydrogenase system by an agent capable of reducing the cytochrome that is made available upon stimulation of the cells. Again there is no clue to the nature of the oxidizing enzymes that replace the cytochrome system in the resting cells.

Perhaps the most serious obstacle to the immediate acceptance of Stannard's and Korr's conclusions is the possibility that at the low levels of oxygen consumption the oxidase system is only partially saturated with substrate and hence considerable amounts could be blocked by inhibitors without affecting the oxygen consumption of the tissue. Such a view has been expressed by Warburg (86), and Commoner (87) has actually demonstrated that cyanide inhibition of yeast respiration is dependent on substrate respiration.

The early work of Keilin showed that whereas in the resting muscle the cytochromes were oxidized, when it became active the bands of the reduced cytochromes appeared. Thus during activity the ratio of reduced to oxidized cytochrome $c$ is higher. It is known that reduced cytochrome $c$ forms a complex with the oxidase (74, 75 ). Since under conditions of activity the oxidase is relatively more saturated with its substrate (reduced cytochrome $c$ ), the respiration of this system should, according to the "under-saturation" concept, be more sensitive to the same concentration of oxidase inhibitor. The "activity" respiration should then be totally sensitive to azide if azide only affects the rate of oxidation of the cytochrome. But Stannard's experiment demonstrated that during activity only the extra oxygen consumption caused by the activity was azidesensitive.

The experiments of Stotz, Altschul, and Hogness (75) on the relations of the oxidase and of cytochrome $c$ on hydroquinone oxidation showed that the rate of oxidation was a function of both components. Thus at a fixed concentration of reduced cytochrome $c$ (oxi- 
dase under-saturated) cyanide does produce an inhibition of hydroquinone oxidation. But if the cytochromes are chiefly in the oxidized state, as they are in the resting tissue, such a decrease in oxygen consumption could be compensated for by increased reduction of the cytochromes. It is therefore important to know whether in the experiments of Stannard and of Korr azide does or does not produce an increased state of reduction of the cytochromes in the resting tissue.

The differences between the characteristics of the respiration of the eggs of various species before and after fertilization have been extensively studied and have been related to the function of the cytochrome system. The results are at present very difficult to interpret, largely because of the tests employed for detecting the oxidase or the cytochrome and because of the problem of permeability of the cells to inhibitors. For example, it appears that in the unfertilized eggs of the sea urchin cytochrome has never been observed spectroscopically, and its respiration is not inhibited by cyanide or azide. After fertilization, however, the respiration is typical of one proceeding through the cytochrome system (see Shapiro, 88). Korr believes the respiration of the unfertilized sea urchin egg to be another case of functional inactivity of the cytochrome system, since these eggs can oxidize $p$-phenylenediamine (see Discussion in Shapiro, 88). Allen (89), on the other hand, using grasshopper eggs, has shown that the activity metabolism is definitely connected with the new development, rather than with "gearing," of the cytochrome system.

A recent paper by Krahl, Keltch, Neubeck, and Clowes (90) on the cytochrome system of sea urchin eggs demonstrates that there is a complete absence of cytochrome $c$ in the unfertilized eggs, but no lack of cytochrome oxidase. On the other hand, even in the fertilized eggs, which are relatively more cyanide- and azide-sensitive, they were still unable to detect any cytochrome $c$. The establishment of the presence of cytochrome oxidase in the unfertilized eggs, although it does not constitute proof, is strong evidence that this compound functions in the respiration of these cells. The authors favor the hypothesis suggested earlier (91) and described by Ball (page 29 ) that the action of cyanide and azide on cellular respiration may be related to the formation of a complex with the oxidase possessing a lower potential than the original oxidase $(92,93)$. The sensitivity or stability of a respiration to azide or cyanide then depends on the potential of the oxidase-inhibitor complex formed and the potentials 
of the available reducing systems. With a given lower potential of the oxidase-inhibitor complex, the lower the potential of the reducing system the less sensitive would be the respiration to the inhibitor. On this basis, a system in which cytochrome $c$ (with a relatively high potential) is the normal reducing agent of the oxidase would be expected to show a high sensitivity to oxidase inhibitors. In the absence of cytochrome $c$ this theory provides an explanation of cyanide or azide stability even when the cyanide may be combining with the oxidase component. Such a theory deserves consideration in the interpretation of all experiments purporting to demonstrate the "by-passing" or non-functioning of the cytochrome-cytochrome oxidase system.

The frontiers of research in the cytochrome problem therefore appear to consist on the biochemical side in the isolation and properties of the individual components, and on the physiological side in the mode of function or non-function of this system in various states of metabolism of tissues.

\section{REFERENCES}

1. MacMunn, C. A., J. Physiol., 8, 57 ( 1887).

2. Keilin, D., Proc. Roy. Soc. (London), B 98, 312 (1925).

3. Keilin, D., Proc. Roy. Soc. (London), B 100, 129 (1926).

4. Theorell, H., Biochem. Z., 285, 207 (1936).

5. Keilin, D., and Hartree, E. F., Proc. Roy. Soc. (London), B 122, 298 (1937).

6. Theorell, H., and Akesson, A., Science, 90, 67 (1939).

7. Zeile, K., and Reuter, F., Z. physiol. Chem., 221, 101 (1933).

8. Sтотz, E., unpublished experiments.

9. Keilin, D., and Hartree, E. F., Proc. Roy. Soc. (London), B 129, 277 (1940).

10. Keilin, D., and Hartree, E. F., Proc. Roy. Soc. (London), B 127, 167 (1940).

11. Stenn, K. G., Discussion, Symposia on Quantitative Biology, Cold Spring Harbor, VII, 119 (1939).

12. Keilin, D., and Hartree, E. F., Proc. Roy. Soc. (London), B 122, 298 (1937).

13. Ketlin, D., Ergebnisse d. Enzymforschung, 2, 239 (1933).

14. Altschul, A. M., and Hogness, T. R., J. Biol. Chein., 124, 25 (1938).

15. Hogness, T. R., Zscheile, F. P., Jr., and Smwell, A. E., Jr., J. Phys. Chem., 41, 379 (1937).

16. Potter, V. R., J. Biol. Chem., 137, 13 (1941).

17. Hill, R., and Keilin, D., Proc. Roy. Soc. (London), B 107, 286 (1930).

18. Zeile, K., and Piutti, P., Z. physiol. Chem., 218, 52 (1933).

19. Theonell, H., Biochem. Z., 298, 242 (1938).

20. Tireorell, H., Biochem. Z., 301, 201 (1939).

21. Zeile, K., and Meyer, H., Z. physiol. Chem., 262, 178 (1939).

22. Coolidge, T. B., J. Biol. Chem., 98, 755 ( 1932 ).

23. Green, D. E., Proc. Roy. Soc. (London), B 114, 423 (1934). 
24. Wurmser, R., and Filitti-Wurmser, S., Compte Rend. Soc. Biol., 127, 471 (1938).

25. Stotz, E., Sidwell, A. E., Jr., and Hogness, T. R., J. Biol. Chem., 124, 11 (1938).

26. BaLl, E. G., Biochem. Z., 295, 262 (1938).

27. Yakushij, E., and Morr, T., Acta Phytochim., 10, 113 (1937).

28. Yakusizi, E., and Okunuki, K., Proc. Imp. Acad. (Tokyo), 17, 38 (1941).

29. Baumberger, J. P., Symposia on Quantitative Biology, Cold Spring Harbor, VII, 195 (1939).

30. Korr, I. M., Symposia on Quantitative Biology, Cold Spring Harbor, VII, 74 (1939).

31. Warburg, O., Biochem. Z., 152, 479 (1924).

32. WARBuRg, O., Biochem. Z., 177, 471 (1926).

33. Warburg, O., Biochem. Z., 189, 354 (1927).

34. Warburg, O., and Negelein, E., Biochem. Z., 214, 64 (1929).

35. Kubowitz, F., and HAAs, E., Biochem. Z., 255, 247 (1932).

36. Warburg, O., and Negelein, E., Biochem. Z., 244, 9 (1932).

37. Warburg, O., and Negelein, E., Biochem. Z., 262, 237 (1933).

38. KeIliN, D., Nature, 132, 783 ( 1933).

39. Fujita, A., and Kodama, T., Biochem. Z., 273, 186 (1934).

40. Keilin, D., Proc. Roy. Soc. (London), B 104, 206 (1928).

41. Warburg, O., and Negelein, E., Biochem. Z., 262, 237 (1933).

42. Warburg, O., Negelein, E., and HaAs, E., Biochem. Z., 266, 1 (1933).

43. Warburg, O., and HaAs, E., Naturwissenschaften, 22, 207 (1934).

44. Melnick, J. L., Science, 94, 118 (1941).

45. Kuвоwiтz, F., Biochem. Z., 292, 221 ( 1937 ).

46. Keilin, D., and Mann, T., Proc. Roy. Soc. (London), B 125, 187 (1938).

47. Cohen, E., and Elvehjem, C. A., J. Biol. Chem., 107, 97 (1934).

48. Yoshikawa, H., J. Biochem. (Japan), 25, 627 (1927).

49. Schultze, M. O., J. Biol. Chem., 129, 729 (1939).

50. Schultze, M. O., J. Biol. Chem., 138, 219 (1939).

51. Graubard, M., Am. J. Physiol., 131, 584 (1941).

52. Altschul, A. M., Abranis, R., and Hogness, T. R., J. Biol. Chem., 130, 427 (1939).

53. Altschul, A. M., Abrams, R., and Hogness, T. R., J. Biol. Chem., 136, 777 (1940).

54. Stern, K. G., Symposia on Quantitative Biology, Cold Spring Harbor, VII, 312 (1939).

55. Straub, F. B., Biochem. J., 33, 787 (1939).

56. v. Euler, H., and Hellströм, H., Z. physiol. Chem., 260, 163 (1939).

57. Alt, H. L., Biochem. Z., 221, 498 ( 1930 ).

58. HaAs, E., Naturwissenschaften, 22, 207 (1934).

59. Hopkins, F. G., Lutwak-ManN, C., and Morgan, E. J., Nature, 143, 556 (1939).

60. Stern, K. G., and Melnick, J. L., Nature, 144, 330 (1939).

61. Horecker, B. L., Stotz, E., and Hogness, T. R., J. Biol. Chem., 128, 251 (1939).

62. Warburg, O., and Christian, W., Biochem. Z., 254, 438 (1932); 257, 492 (1933).

63. Warburg, O., and Christian, W., Biochem. Z., 266, 377 (1933).

64. Theorell, H., Biochem. Z., 288, 317 (1936).

65. HaAs, E., Horecker, B. L., and Hogness, T. R., J. Biol. Chem., 136, 747 (1940).

66. Lockhart, E. E., and Potrer, V. R., J. Biol. Chem., 137, 1 (1941). 
67. Adler, E., v. Euler, H., and Hellström, H., Arkiv. Kemi, Mineral. Geol., $12 B$, No. 38 (1937).

68. Green, D. E., and Dewan, J. G., Biochem. J., 32, 626 (1938).

69. Haivthorne, J. R., and Harrison, D. C., Biochem. J., 33, 1573 (1939).

70. Corran, H. S., Green, D. E., and Straub, F. B., Biochem. J., 33, 793 (1939).

71. Keilin, D., Proc. Roy. Soc. (London), B 106, 418 (1930).

72. Stotz, E., Harrer, C. J., Schultze, M. O., and King, C. G., J. Biol. Chem., 122, 407 (1938).

73. Keilin, D., and Hartree, E. F., Proc. Roy. Soc. (London), B 125, 171 (1938).

74. Stotz, E., Sidwell, A. E., and Hogness, T. R., J. Biol. Chem., 124, 733 (1938).

75. Stotz, E., Altschul, A. M., and Hogness, T. R., J. Biol. Chem., 124, 745 (1938).

76. Junowicz-Kochalaty, R., and Hogness, T. R., J. Biol. Chem., 129, 569 (1939).

77. Stотz, E., J. Biol. Chem., 131, 555 (1939).

78. Potter, V. R., and Dubois, K. P., J. Biol. Chem., 140, Scientific Proceedings XXXV, cii (1941).

79. Harrer, C. J., and King, C. G., J. Biol. Chem., 138, 111 (1941).

80. Stiehler, R. D., and Flexner, L. B., J. Biol. Chem., 126, 603 (1938).

81. Flexner, L. B., and Stiehler, R. D., J. Biol. Chem., 126, 619 (1938).

82. Flexner, J. B., Flexner, L. B., and Strauss, W. L., Jr., Proc. Am. Physiol. Soc., 1941, p. 90.

83. Stannard, J. N., Symposia on Quantitative Biology, Cold Spring Harbor, VII, 394 (1939).

84. Stannard, J. N., Am. J. Physiol., 122, 379 (1938).

85. Stannard, J. N., Am. J. Physiol., 126, 196 (1939).

86. Warburg, O., Biochem. Z., 189, 354 (1927).

87. Commoner, B., J. Cell. Comp. Physiol., 13, 121 (1939).

88. Shapiro, H., Symposia on Quantitative Biology, Cold Spring Harbor, VII, $406(1939)$.

89. Allen, T. H., J. Cell. Comp. Physiol., 16, 149 (1940).

90. Krahl, M. E., Keltch, A. K., Neubeck, C. E., and Clowes, G. H. A., J. Gen. Physiol., 24, 597 (1941).

91. BalL, E. G., in discussion of the paper by E. S. G. Barron, Symposia on Quantitative Biology, Cold Spring Harbor, VII, 154 (1939).

92. Barron, E. S. G., J. Biol. Chem., 121, 285 (1937).

93. Clark, W. M., Taylor, J. H., Davies, T. H., and Vestling, C. S., J. Biol. Chem., 135, 543 (1940). 


\title{
Phosphorylation of Carbohydrates
}

\author{
CARL F. CORI \\ Washington University School of Medicine, St. Louis
}

\begin{abstract}
T 7 HE METABOLISM of carbohydrate in animal tissues is made up 1 of a series of enzymatic reactions in which phosphate plays an essential role. What is usually referred to as the phosphate cycle can be divided into four parts: the uptake of inorganic phosphate, the intramolecular migration of phosphate groups, the transfer of phosphate groups from one molecule to another (transphosphorylation), and the regeneration of inorganic phosphate.
\end{abstract}

\section{Uptake of Inorganic Phosphate}

The only reaction leading to the uptake of inorganic phosphate that is definitely known to be enzymatic is the phosphorylation of glycogen and starch. The uptake of inorganic phosphate which is associated with the oxidation of phosphoglyceraldehyde is presumably non-enzymatic, and the same may be true of the uptake of inorganic phosphate associated with the oxidation of pyruvate.

In the phosphorylation of glycogen the $\mathrm{C}-\mathrm{O}-\mathrm{C}$ bond of the $1-4$ glucosidic chain is replaced by the $\mathrm{C}-\mathrm{O}-\mathrm{P}$ bond of glucose-1phosphate. This reaction is reversible, and from the position of the equilibrium it may be calculated that the change in free energy is very small. This may be interpreted to mean that the ester linkage in glucose-1-phosphate is nearly equivalent to the glucosidic linkage in the large polysaccharide molecule.

When phosphate is replaced by water, as in the hydrolysis of glycogen or starch by diastase, the reaction seems to be largely irreversible; that is, the end products of diastatic activity, maltose and glucose, even when added to diastase in high concentrations, are not polymerized to glycogen or starch. Glucose, in order to undergo enzymatic polymerization, must first be phosphorylated. Reversibility is thus clearly connected with the introduction of a phosphate group into the polysaccharide molecule. The position of the equilibrium at physiological $\mathrm{pH}$ is about 77 per cent to the glycogen side (see Table 2), and is determined by the concentration of the divalent ions of orthophosphate and glucose-1-phosphate. Since the 
second dissociation constant of these two acids is different, the position of the equilibrium changes with $\mathrm{pH}(1,2)$.

The enzymatic phosphorylation of glycogen and starch is of interest from the standpoint of the configuration of these polysaccharide molecules. There is considerable evidence, based on the hydrolysis of methylated starch and glycogen, that these polysaccharides contain other than the prevalent 1-4 glucosidic linkages. At points at which a branching of chains occurs, a 1-6 glucosidic linkage has been postulated. Starch appears to be made up of relatively straight chains consisting of 24 to 30 glucose units; this explains its ability to assume a crystalline structure and to exhibit well-defined x-ray diffraction patterns. Glycogen seems to be made up of relatively short chains (consisting of 12 to 18 glucose units), with many branchings which give it the properties of greater solubility and lack of crystallizability. Judging from the properties of various plant starches, transitions exist between these two extremes. These various forms of polysaccharide raise the problem of enzyme specificity. The questions are whether one and the same enzyme splits (or builds up) both the 1-4 and the 1-6 glucosidic linkages and what determines the special configuration of the polysaccharide synthesized. It is to be noted here that phosphorylases cannot polymerize glucose-1-phosphate unless a small amount of polysaccharide is added to prime the reaction (1). However, the nature of the polysaccharide synthesized seems to be solely determined by the type of phosphorylase used and not by the nature of the activating polysaccharide. For example, muscle phosphorylase, when primed with liver glycogen, synthesizes a typical starch in vitro, and liver phosphorylase, when primed with plant starch, synthesizes glycogen (3). Important also in this connection is the fact that the same enzyme, muscle phosphorylase, can synthesize both starch and glycogen, the former in vitro and the latter in the intact cell. The difference in activity of the muscle phosphorylase in the two situations has not been explained, but it suggests that unknown environmental factors and perhaps the physical state of the enzyme have something to do with the nature of the polysaccharide which is formed.

As has been stated, the phosphorylation of glycogen is an enzymatic reaction which provides for the entrance of inorganic phosphate into the phosphate cycle. All other reactions leading to the uptake of inorganic phosphate are linked with oxidations. The reaction between orthophosphate and phosphoglyceraldehyde during oxidation of the latter to phosphoglyceric acid has been elucidated 
by Warburg and his school (4). Lipmann (5) has described a bacterial enzyme system in which inorganic phosphate is taken up and acetylphosphate is formed as an intermediate of pyruvate oxidation. In both cases the phosphate group taken up during oxidation is transferred by the adenylic acid system to suitable phosphate acceptors.

The oxidation of pyruvate in various animal tissues is also linked with the uptake of inorganic phosphate, and the same has been shown to be true for certain steps of the citric acid cycle, particularly for the oxidation of succinic to fumaric acid (6). The primary phosphorylation products formed in these cases have not been identified. In dialyzed and suitably supplemented tissue dispersions or extracts, the inorganic phosphate taken up during oxidation of pyruvate is transferred by the adenylic acid system to glucose, which is converted to hexosediphosphate. It was noted, however, that when no glucose was added to the system, a small amount of an easily hydrolyzable phosphorus compound was formed. This compound has recently been identified in our laboratory in collaboration with Dr. Ochoa. In large-scale experiments with dialyzed rat liver dispersion and with glutamate, pyruvate, or succinate as oxidizable substrate we have isolated inorganic pyrophosphate as the crystalline sodium salt. The orthophosphate which disappeared corresponded in amount to the pyrophosphate formed.

It is too early to evaluate the significance of this observation. There is the possibility that the pyrophosphate group occurs in some organic combination which is split during the process of isolation. It is fairly certain, however, that the pyrophosphate does not originate from adenosinetriphosphate, since we have found no enzyme in liver preparations which splits added adenylpyrophosphate to adenylic acid and inorganic pyrophosphate. Another possibility is that the pyrophosphate group has nothing to do with the primary phosphorylation product which is formed during the oxidation of the substrate, but is the result of phosphorylation of orthophosphate.

The phosphorylation of glucose and other phosphate acceptors which is connected with the oxidation of pyruvate has been termed "aerobic phosphorylation" to signify that the energy for the formation of the phosphate bond comes from oxidations. Table 1 illustrates the quantitative relationship between oxygen consumption and phosphorylation.

This experiment shows that the dialyzed heart extract supplemented with magnesium ions, inorganic phosphate, and a trace of 
adenylic acid has practically no basal oxygen consumption and that addition of 2 micromoles of succinic acid, a catalytic amount, has very little effect on oxygen consumption. The addition of 50 micromoles of glucose had a very marked effect on oxygen consumption,

Table 1.-Glucose balance in dialyzed heart muscle extract ${ }^{*}$ (1 cc. of extract supplemented with $\mathrm{Mg}^{++}$ions, inorganic phosphate, and a trace of adenylic acid. Incubated 60 minutes at $37^{\circ} \mathrm{C}$. All values are expressed in micromoles.)

\begin{tabular}{lcccc}
\hline Addition & $\begin{array}{c}\text { Glucose } \\
\text { disappearing }\end{array}$ & $\begin{array}{c}\text { Oxygen } \\
\text { consumed }\end{array}$ & $\begin{array}{c}\text { Phosphate } \\
\text { esterified }\end{array}$ & $\begin{array}{c}\text { Total glucose } \\
\text { accounted for }\end{array}$ \\
\hline $\begin{array}{l}\text { None } \\
\text { 2 succinate }\end{array}$ & 1.5 & & \\
$\begin{array}{l}\text { 5 glucose }+ \\
2 \text { succinate }\end{array}$ & 24.4 & 22.3 & 36.1 & $3.5+18=21.5$ \\
\hline
\end{tabular}

* J. Biol. Chem., 137, 343 (1941).

and there can be no doubt that glucose was the substrate undergoing oxidation. Determination of the respiratory quotient in other experiments showed that it was unity for added glucose and 1.25 for added pyruvate. It is to be noted that an oxygen consumption of 22 micromoles corresponds to one-sixth as much glucose, that is to 3.5 micromoles, while the glucose which actually disappeared according to sugar analysis was 24.4 micromoles. The glucose which disappeared without being oxidized was largely recovered as hexosediphosphate, 36 micromoles of phosphate esterified corresponding to 18 micromoles of glucose. Lactic acid formation was not determined. The balance indicates that for each mole of glucose oxidized an additional 6 moles of glucose disappear, 5 of which are present as phosphate ester. This means that about one atom of phosphate is esterified for each atom of oxygen consumed. Ochoa (7) and Belitzer and Tsibakova (8) observed even higher ratios, namely, from 2 to 3 atoms of phosphate esterified for each atom of oxygen consumed. This would indicate that not only the primary removal of hydrogen from the substrate but also one or even two subsequent hydrogen transfers over intermediate catalysts may cause phosphorylation.

Aerobic phosphorylation is a mechanism by which oxidative energy is utilized in the cell. The oxidative energy is converted into phosphate bond energy, to use Lipmann's (5) terminology, and the adenylic acid system serves as the mediator of this energy transfer. When glucose is the phosphate acceptor, the system, once started, is 
self-perpetuating. The phosphorylation of glucose enables it to undergo oxidation by way of triosephosphate and pyruvate, and this oxidation causes further phosphorylation of glucose, thus providing new substrate for oxidation and so on.

During recovery of muscle from work, oxidative energy is also converted into phosphate bond energy; that is, the phosphocreatine which breaks down during muscular contraction is reformed largely at the expense of oxidation, and the phosphorylation of glucose which is supplied by the blood stream provides the necessary substrate for the resynthesis of the glycogen lost during contraction.

\section{Intramolecular Migration of Phosphate Groups}

The first enzymatic reaction of this type was described by Meyerhof and Kiessling (9), namely, the conversion of glyceric acid-3- to glyceric acid-2-phosphate. This reaction was shown to be reversible, and the assumption that one is dealing with an intramolecular migration of the phosphate group was confirmed by the use of radioactive phosphorus.

Another reaction of this type is the conversion of glucose-1- to glucose-6-phosphate. This reaction was at first regarded as irreversible, but more recent work has shown that it can be reversed under suitable experimental conditions; that is, glucose-6-phosphate can be converted to glucose-1-phosphate and then to glycogen (10). To study the equilibrium of this reaction it was necessary to separate the enzyme which catalyzes this reaction from interfering enzymes which upset the equilibrium by acting either on glucose-1- or glucose-6-phosphate. With such a purified enzyme preparation 94 per cent of added glucose-1-phosphate is converted to an ester which was isolated and identified as glucose-6-phosphate. Fructose-6-phosphate was absent, because Lohmann's enzyme (11), which catalyzes the reversible reaction between glucose-6- and fructose-6-phosphate, had been removed. Conversely, when pure glucose-6-phosphate was added to the enzyme, 6 per cent of glucose-1-phosphate was formed; that is, the same equilibrium was reached from either side. From the equilibrium constant ( $\mathrm{K}=15.7$ at $\mathrm{pH} 7$ and $25^{\circ} \mathrm{C}$.) it may be calculated that the change in standard free energy amounts to about -1600 calories.

When barium ions and phosphorylase were added to this system, glucose-6-phosphate was converted to glycogen. As shown in Table 2 , the position of the equilibrium of the second reaction is unfavorable for glycogen synthesis, because only a small amount of glucose- 
Table 2.-Position of equilibria at $\mathrm{pH} 7$ and $25^{\circ}$

Glycogen+inorganic phosphate (77 per cent) $\rightleftarrows$ glucose-1-phosphate (23 per cent) (1)

Glucose-1-phosphate ( 6 per cent) $\rightleftarrows$ glucose-6-phosphate $(9+$ per cent)

Glucose-6-phosphate (70 per cent) $\rightleftarrows$ fructose-6-phosphate (30 per cent)

1-phosphate is formed. The overall reaction cannot progress very far to the glycogen side, but when barium ions are added, which cause precipitation of the inorganic phosphate set free when reaction 1 goes to the left, up to 40 per cent of added glucose-6phosphate can be converted to glycogen. Such an experiment is shown in Table 3.

Table 3.-Glycogen formation from barium salt of glucose-6phosphate with dialyzed muscle extract

(Extract $+\mathrm{Mg}^{++}$ions + catalytic amounts of glycogen and adenylic acid.

Incubated at $30^{\circ} \mathrm{C}$.)

\begin{tabular}{lcc}
\hline \multicolumn{1}{c}{ Type of extract } & Time of incubation & $\begin{array}{c}\text { 6-ester converted } \\
\text { to glycogen }\end{array}$ \\
\hline Original & minutes & per cent \\
& 60 & 5.0 \\
Concentrated sevenfold & 180 & 15.1 \\
& 10 & 3.1 \\
Concentrated, but without addition of & 60 & 9.9 \\
adenylic acid and glycogen & 180 & 1.2 \\
\hline
\end{tabular}

Muscle extract, prepared in the usual manner and dialyzed free of inorganic phosphate, shows only a slight activity when the barium salt of glucose-6-phosphate is added. When the same extract was concentrated sevenfold by freezing and by drying in vacuo, the activity was markedly increased, since up to 33 per cent of the added glucose-6-phosphate was converted to glycogen. The glycogen formation was measured by (1) the amount of inorganic phosphate set free, which precipitates as the barium salt because of the addition of barium ions; (2) the increase in glycogen, which is somewhat less than the increase calculated from the inorganic phosphate because of the presence of diastase in the extract; and (3) the color reaction with iodine, which is blue for the polysaccharide formed by muscle phosphorylase. As a control procedure, the addition of adenylic acid and of the glycogen necessary for the priming of the reaction 
was omitted, and it may be seen that no conversion of glucose-6phosphate to glycogen took place.

Colowick and Sutherland have also been able to convert glucose to glycogen in vitro by the addition of hexokinase and adenosinetriphosphate to the concentrated muscle extract. It can be shown that in this case glucose is first converted to glucose-6-phosphate at the expense of the labile phosphate groups of adenosinetriphosphate.

Glucose-6-phosphate is thus an important intermediate of carbohydrate metabolism; it is formed from glycogen via glucose-1phosphate and it can also be formed by direct phosphorylation of glucose in position 6 . The latter reaction is the so-called hexokinase reaction which was described by Meyerhof (12) and v. Euler and Adler (13).

\section{TRANSPHOSPHORYLATION}

All known transphosphorylation reactions involve adenosinemonoor adenosinediphosphate; these nucleotides act in catalytic amounts as acceptors of phosphate from such substances as phosphopyruvate, acetylphosphate, and 1,3-diphosphoglycerate and are thus converted to adenosinedi- and triphosphate respectively. These polyphosphates then serve in a second enzyme reaction as phosphate donors to a number of organic molecules, such as glucose, fructose, mannose, fructose-6-phosphate, glycerol, creatine, adenosine, and probably others. The enzymes which transfer the phosphate group from the polyphosphates are specific with respect to the acceptors. For example, yeast contains enzymes that phosphorylate glucose, fructose, mannose, and adenosine, but no enzyme that phosphorylates creatine.

Extract of skeletal muscle has little or no hexokinase activity. Since this can hardly be true of intact muscle, it may be due to destruction, inhibition, or poor extractability of the enzyme. Other tissues, such as brain, heart, liver, kidney, and retina yield active extracts. The hexokinase in these tissue extracts has not been separated from other enzymes, but in the case of yeast such a separation has been effected.

Colowick and Kalckar (14) have recently studied the reaction between adenosinetriphosphate and glucose with purified yeast hexokinase. Only one of the labile phosphate groups of adenosinetriphosphate is transferred to glucose; that is, the reaction products that were identified are adenosinediphosphate and glucose-6-phosphate. When adenosinediphosphate is substituted for adenosinetriphosphate, no reaction with glucose takes place, but when a heat- 
stable protein of muscle is added to the system, hexokinase is able to transfer the labile phosphate group of adenosinediphosphate, the reaction products in this case being adenylic acid and glucose-6phosphate. This is illustrated in Tables 4 and 5 .

Table 4.-Reaction of adenosinetriphosphate with glucose (Hexokinase and $\mathrm{Mg}^{++}$in all samples. $\mathrm{T}=30^{\circ} \mathrm{C}$.)

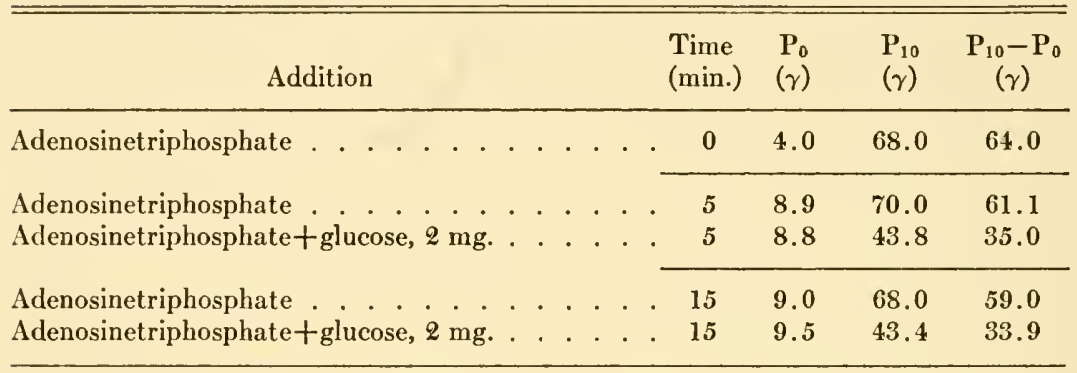

The difference between the $\mathrm{P}_{0}$ and $\mathrm{P}_{10}$ value (initial and ten-minute hydrolysis values in normal sulfuric acid) corresponds to the amount of labile phosphate added as adenosinetriphosphate. With a purified hexokinase preparation of yeast, no appreciable reaction takes place

Table 5.-Necessity of heat-stable muscle protein for reaction of adenosinediphosphate with glucose

(Hexokinase and $\mathrm{Mg}^{++}$in all samples. Time, 5 minutes. $\mathrm{T}=30^{\circ} \mathrm{C}$.)

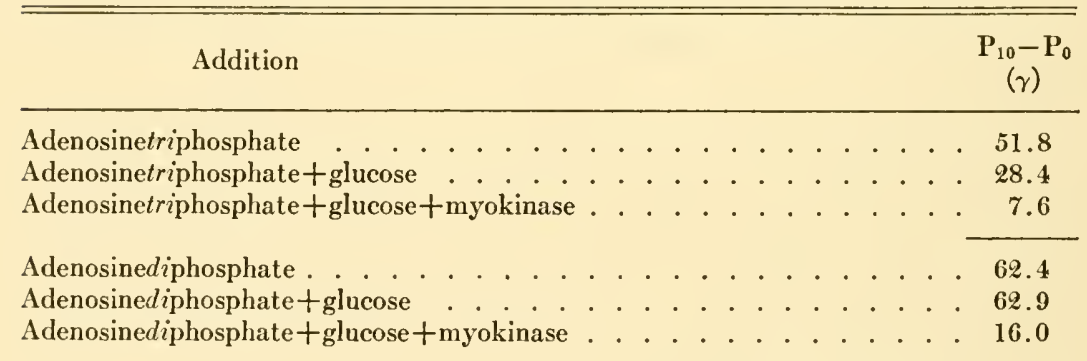

when adenosinetriphosphate alone is added; when glucose is also added, approximately half of the labile phosphate of adenosinetriphosphate disappears. The reaction is a rapid one, since it is nearly completed during five minutes of incubation at $30^{\circ} \mathrm{C}$. Table 5 shows the effect of a heat-stable protein of muscle which has been named 
"myokinase" by Colowick and Kalckar and which, when added in catalytic amounts, enables hexokinase to transfer the labile phosphate group of adenosinediphosphate to glucose. It may be seen that with adenosinetriphosphate as phosphate donor approximately half of the labile phosphate disappears when hexokinase alone is added, and that with the further addition of a few micrograms of muscle factor almost all the labile phosphate disappears. With adenosinediphosphate as phosphate donor no reaction with glucose takes place until the muscle protein is added.

In extracts of mammalian tissues, such as kidney, heart, and brain, the hexokinase reaction is generally followed by a reaction between adenosinetriphosphate and fructose-6-phosphate, yielding fructose-1, 6-diphosphate or Harden-Young ester. In the intact cell, however, particularly in muscle, where this has been studied in detail, fructose-6-phosphate does not react rapidly with adenosinetriphosphate. This is borne out by the fact that hexosemonophosphate, the equilibrium mixture of glucose- and fructose-6-phosphate, is a normal constituent of muscle and that it can increase considerably under certain experimental conditions without any increase in the formation of lactic acid (15). This indicates that the reaction between fructose-6-phosphate and adenosinetriphosphate in intact muscle is a limiting factor as regards the rate at which lactic acid is formed and carbohydrate is oxidized. It is not yet known whether the system for the direct oxidation of glucose-6-phosphate which has been found by Warburg in yeast is significant for mammalian tissues; it would in any case lead to the formation of triosephosphate and hence of pyruvic acid and thus join the main path of carbohydrate breakdown.

The reaction between fructose-6-phosphate and adenosinetriphosphate has not been studied in detail, and the enzyme that catalyzes this reaction has not been purified. The reaction has been regarded as irreversible. Lohmann (11), however, has reported that muscle extract splits off phosphate from position 1 when fructose-1, 6-diphosphate and magnesium ions are added. Recent experiments carried out in our laboratory with Dr. Ochoa have shown that Harden-Young ester added to liver extract is converted in quantitative yield to glucose. This involves dephosphorylation in position 1 , conversion of fructose-6- to glucose-6-phosphate, and splitting of the latter by liver phosphatase to glucose and inorganic phosphate. We have repeatedly convinced ourselves that liver phosphatase forms 
only glucose from the equilibrium mixture of fructose- and glucose6-phosphate.

When fructose is added to liver or kidney extract, it is also converted to glucose. This involves phosphorylation of fructose and splitting of the glucose-6-phosphate in equilibrium with fructose-6phosphate. Table 6 illustrates an experiment in which an aerobic phosphorylation of fructose took place with partial conversion to glucose. In this experiment glutamate was used as oxidizable substrate, which caused a considerable consumption of oxygen. The respiration, as in other cases of aerobic phosphorylation, serves here for the regeneration of adenosinetriphosphate, the phosphate donor

\section{Table 6.-Aerobic conversion of fructose to glucose in dialyzed rat} liver dispersion

(All samples contained $0.2 \mathrm{mg} . \mathrm{Mg}^{++}$, a catalytic amount of adenosinetriphosphate, and $0.025 \mathrm{M}$ phosphate buffer of $\mathrm{pH} 7.3$, in a volume of $1.4 \mathrm{cc}$. Incubated 60 minutes at $37^{\circ} \mathrm{C}$.)

\begin{tabular}{|c|c|c|c|c|c|c|c|}
\hline $\begin{array}{c}\text { Substrate } \\
\text { of } \\
\text { oxidation }\end{array}$ & $\begin{array}{l}\text { Phos- } \\
\text { phate } \\
\text { acceptor }\end{array}$ & $\mathrm{NaF}$ & $\begin{array}{l}\text { Oxygen } \\
\text { con- } \\
\text { sumed }\end{array}$ & $\begin{array}{l}\text { Phosphate } \\
\text { esterified }\end{array}$ & $\begin{array}{l}\text { Fructose } \\
\text { disap- } \\
\text { pearing }\end{array}$ & $\begin{array}{c}\text { Glu- } \\
\text { cose } \\
\text { formed }\end{array}$ & $\begin{array}{l}\text { Fructose } \\
\text { converted } \\
\text { to glucose }\end{array}$ \\
\hline & & $M \times 10^{-3}$ & $\mathrm{cmm}$. & mg. & mg. & mg. & per cent \\
\hline $\begin{array}{l}\text { None } \\
\text { Glutamate }\end{array}$ & $\begin{array}{l}\text { none } \\
\text { none }\end{array}$ & $\begin{array}{r}20 \\
0\end{array}$ & $\begin{array}{l}28 \% \\
962\end{array}$ & $\begin{array}{l}0.22 \\
0.05\end{array}$ & & & \\
\hline Glutamate & none & 20 & 950 & 0.49 & & & \\
\hline Glutamate & fructose & 0 & 932 & 0.14 & 3.42 & 2.67 & 78 \\
\hline Glutamate & fructose & 20 & 938 & 1.10 & 4.17 & 1.37 & 33 \\
\hline
\end{tabular}

to fructose. When no fluoride is added only a small amount of phosphate ester accumulates, and most of the fructose that disappears is converted to glucose. Fructose and glucose were determined by separate methods. In the presence of fluoride, dephosphorylation is inhibited and consequently more hexosephosphate esters accumulate and less glucose is formed. When no phosphate acceptor is added, a small amount of inorganic phosphate is esterified, especially when fluoride is added. This compound is almost exclusively pyrophosphate, as has been mentioned previously.

The mechanism of the conversion of fructose to glucose is shown in Table 7, the enzyme system consisting of adenosinetriphosphate, kidney phosphatase prepared by Albers' method, and yeast hexokinase-which also contains Lohmann's enzyme (11). If hexokinase is not added, neither adenosinetriphosphate nor fructose disappears. When phosphatase is omitted, all the added adenosine- 
triphosphate and a corresponding amount of fructose disappear, but practically no glucose is formed. The formation of glucose is clearly dependent on the addition of phosphatase. The conversion of fructose to glucose is another example of the utilization of oxidative energy in the cell by way of the phosphate cycle.

Table 7.-Conversion of fructose to glucose by a purified enzyme system

(The complete system consisted of $5 \mathrm{mg}$. of hexokinase, $0.05 \mathrm{mg}$. of "muscle factor," $10 \mathrm{mg}$. of phosphatase, $0.2 \mathrm{mg}$. of $\mathrm{Mg}^{++}, 3 \mathrm{mg}$. of fructose, $0.33 \mathrm{mg}$. of labile phosphate (as adenosinetriphosphate), and $0.025 \mathrm{M}$ veronal buffer of $\mathrm{pH} 7.5$, in a total volume of 1.3 cc. Incubated 60 minutes at $37^{\circ} \mathrm{C}$.)

\begin{tabular}{lcccc}
\hline Sample & $\begin{array}{c}\text { Adenosinetri- } \\
\text { phosphate dis- } \\
\text { appearing }\end{array}$ & $\begin{array}{c}\text { Fructose } \\
\text { disappearing }\end{array}$ & $\begin{array}{c}\text { Glucose } \\
\text { formed }\end{array}$ & $\begin{array}{c}\text { Fructose } \\
\text { converted to } \\
\text { glucose }\end{array}$ \\
\hline & $\begin{array}{l}\text { mg. labile } \\
\text { phosphate }\end{array}$ & mg. & mg. & per cent \\
No adenosinetriphosphate & 0 & 0 & 0 & \\
No hexokinase . . . . . & 0.07 & 0 & 0 & \\
No phosphatase. . . . . & 0.29 & 1.2 & 0.07 & 6 \\
Complete. . . . . . . & 0.33 & 1.1 & 0.48 & 44 \\
\hline
\end{tabular}

It may be emphasized at this point that all the reactions of the phosphate cycle except the phosphorylation of pyruvic acid by adenosinetriphosphate have now been shown to be reversible. When lactic or pyruvic acid is converted to carbohydrate, phosphopyruvic acid is apparently formed in an indirect way, probably from a fourcarbon dicarboxylic acid such as malate or fumarate, both of which are assumed to be intermediates in the oxidation of pyruvate. Kalckar (16) has shown that when malate or fumarate is added to kidney extract under aerobic conditions, phosphopyruvic acid is formed.

\section{Regeneration of Inorganic Phosphate}

The formation of phosphopyruvate has just been mentioned. The reverse reaction, the dephosphorylation of phosphopyruvate, was originally shown to consist in a transfer of phosphate from phosphopyruvate to adenylic acid, with formation of pyruvate and adenosinetriphosphate. The reaction proceeds rapidly with catalytic amounts of adenylic acid, provided the adenylic acid is regenerated, either by phosphate transfer from adenosinetriphosphate to some suitable phosphate acceptor such as creatine, or by dephosphorylation of adenosinetriphosphate by adenylpyrophosphatase. It was 
later shown by Pillai (17), and confirmed by us and recently also by Parnas (18), that there is apparently a second mechanism for the dephosphorylation of phosphopyruvate. This conclusion is based on the observation that dialyzed and aged muscle extract or an acetone powder of muscle extract which is unable to split adenosinetriphosphate, and thus to regenerate adenylic acid, can still split phosphopyruvate when a catalytic amount of adenosinetriphosphate is added. That one is dealing with a different type of reaction is shown by the fact that in such extracts adenylic acid cannot replace adenosinetriphosphate.

Another reaction that leads to the regeneration of inorganic phosphate is the splitting of adenosinetriphosphate by adenylpyrophosphatase. There is reason to believe that the activity of this enzyme is increased during muscular contraction (19). Adenylpyrophosphatase, which is found in most tissues, plays an important regulatory function; by converting adenosinetriphosphate to adenylic acid it can overcome the "bottleneck" which is created in the phosphate cycle by a lack of phosphate acceptors. In addition, it is possible that the reaction described by Pillai, the direct dephosphorylation of phosphopyruvate, plays a physiological role.

In some experiments with tissue slices the phosphate cycle is so perfectly adjusted that the concentration of inorganic phosphate remains virtually unchanged, and this has given rise to the erroneous assumption that one is dealing with a non-phosphorylating glycolysis. Dr. Ochoa in our laboratory has recently investigated the glycolysis in brain, which has been regarded by some workers as a tissue with non-phosphorylating glycolysis. In the past most of the work was done with brain slices or brei because with brain extracts the formation of lactic acid was very feeble. Geiger (20) made the significant observation that when a brain extract which forms little lactic acid is diluted, a rapid lactic acid formation sets in. This is due to the fact that an inhibitor is present in brain extract, the effect of which is nullified by dilution. Ochoa (21), who confirmed Geiger's observation, was able to show that all the reactions which are characteristic for phosphorylating glycolysis occur in this dilute brain extract. One illustrative experiment is shown in Table 8. The amount of lactic acid formed for an extract corresponding to only $40 \mathrm{mg}$. of tissue is quite large, as good as or better than is obtained with other tissue extracts. Glucose, hexosemono-, and hexosediphosphate form about equal amounts of lactic acid and the changes in lactic acid and inorganic phosphate correspond to the equations 
given at the bottom of the table. There can be no doubt that lactic acid is formed here by a phosphorylating mechanism. This is not to imply that such a mechanism is the only one that has been invented by nature for the degradation of carbohydrate, but so far as animal tissues are concerned it would seem that the burden of proof is on those who claim that a non-phosphorylating glycolysis exists.

\section{Table 8.-Glycolysis in rat brain extract}

(0.2 cc. of extract (equivalent to $40 \mathrm{mg}$. of brain) were made up to $2 \mathrm{cc}$. with addition of $\mathrm{Mg}^{++}$, phosphate-bicarbonate buffer, and catalytic amounts of adenosinetriphosphate and cozymase. Incubated 90 minutes at $38^{\circ}$ C. From J. Biol. Chem., 141, 245,1941 .)

\begin{tabular}{|c|c|c|c|}
\hline \multirow[b]{2}{*}{ Substrate } & \multicolumn{2}{|c|}{ Lactic acid formed } & \multirow{2}{*}{$\begin{array}{l}\text { - Change in inor- } \\
\text { ganic phosphate }\end{array}$} \\
\hline & $\begin{array}{c}\text { Determined } \\
\text { manometrically }\end{array}$ & $\begin{array}{l}\text { Determined } \\
\text { chemically }\end{array}$ & \\
\hline None & $\begin{array}{l}\text { mg. } \\
0.15\end{array}$ & $\begin{array}{l}\text { mg. } \\
0.19\end{array}$ & mg. \\
\hline Glucose $(0.028 \mathrm{MI})^{*}$. . . . . . & 1.22 & 1.58 & -0.36 \\
\hline Hexose monophosphate $(0.010 \mathrm{M}) \dagger$ & 1.17 & 1.38 & +0.03 \\
\hline Hexose diphosphate $(0.017 \mathrm{M}) \ddagger$. & 1.15 & 1.20 & +0.35 \\
\hline
\end{tabular}

$* 2$ hexose $+2 \mathrm{H}_{3} \mathrm{PO}_{4}=2$ lactic acid +1 hexose diphosphate.

$\dagger 2$ hexose monophosphate $=2$ lactic acid +1 hexose diphosphate.

$\ddagger 1$ hexose diphosphate $=2$ lactic acid $+2 \mathrm{H}_{3} \mathrm{PO}_{4}$.

Table 9 summarizes the essential reactions of the phosphate cycle. One important feature of this scheme is that the concentration of inorganic phosphate has a marked influence on the rate of enzymatic reactions. This is obvious in the case of the first reaction, since whether glycogen will be broken down or synthesized depends entirely on the relative concentrations of inorganic phosphate and glucose-1-phosphate. It may also be pointed out that the oxidation of triosephosphate and of pyruvate cannot occur in the absence of inorganic phosphate and that its concentration therefore has a marked effect on the rate of oxidation. Another feature of the scheme which needs to be emphasized is that the rate of oxidation is also dependent on the availability of a phosphate acceptor, because of the fact that oxidation and phosphate transfer are coupled reactions. It is for this reason that the addition of adenylic acid is often found to have a marked stimulating effect on carbohydrate oxidation in an enzyme system. Finally, other phosphate acceptors, particularly creatine, and the dephosphorylating enzymes (phosphatase, adenyl- 
pyrophosphatase), play an important role as regulators of the concentration of inorganic phosphate.

\section{Table 9.-Reactions of the phosphate cycle}

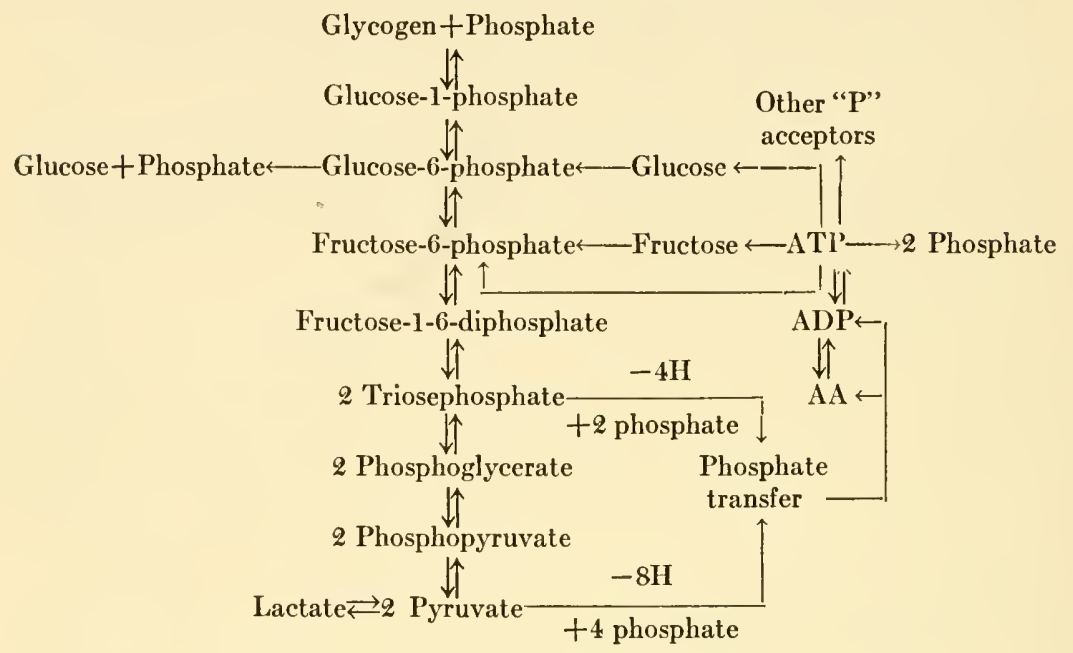

\section{SUMMARY}

This brief presentation of the phosphate cycle is of course far from a complete picture. Its elements are the individual enzymatic reactions. If an enzyme has been isolated from the tissues, if the reaction product or products have been identified, if the kinetics of the reaction and the role of coenzymes, activators, and inhibitors are known, we are, I believe, on solid ground. Several of the individual enzymatic reactions comprising the phosphate cycle have been studied in this manner; others remain to be studied in greater detail.

It is also possible to combine a number of individual enzymatic reactions in the test tube and to reproduce overall effects, such as the polymerization of glucose to glycogen or the conversion of fructose to glucose. The coupling between respiration and phosphorylation has given us an insight into the mechanism of energy transfer in the cell. There is still an essential element lacking in this picture, which in the absence of a better definition might be called the regulatory function of the cell. There can be no doubt that mechanisms exist in the intact cell which regulate the rate and direction of individual enzymatic reactions and which lead to a high degree of integration 
of overall effects. The next approach is, perhaps, a study of the mechanisms underlying this regulatory function. The mechanism of action of a number of hormones is still obscure; all that can be said at present is that they are part of the regulatory mechanism of the cell rather than essential constituents of enzyme systems.

\section{REFERENCES}

1. Cori, G. T., and Cori, C. F., J. Biol. Chem., 135, 733 (1940).

2. Hanes, C. S., Proc. Roy. Soc. (London), B 129, 174 (1940).

3. Baer, R. S., and Cori, C. F., J. Biol. Chem., 140, 111 (1941).

4. Warburg, O., and Christian, W., Biochem. Z., 303, 40 (1939).

5. LipmanN, F., Advances in Enzymology, 1, 99 (New York, 1941).

6. Colowick, S. P., Kalckar, H. M., and Cori, C. F., J. Biol. Chem., 137, 343 (1941).

7. ОсноA, S., J. Biol. Chem., 138, 751 (1941).

8. Belitzer, V. A., and Tsibakova, E. F., Biokimia, 4, 516 (1939).

9. Meyerhof, O., and Kiessling, W., Biochem. Z., 276, 239 (1935).

10. Sutherland, E. W., Colowick, S. P., and Cori, C. F., J. Biol. Chem., 140, 309 (1941).

11. Lohmann, K., Biochem. Z., 262, 137 (1933).

12. MeYerhoF, O., Biochem. Z., 183, 176 (1927); Naturwissenschaften, 23, 850 (1935).

13. v. Euler, H., and Adrer, E., Z. physiol. Chem., 235, 122 (1935).

14. Colowick, S. P., and KalCKar, H. M., J. Biol. Chem., 137, 789 (1941); 140, xxix (1941).

15. Corr, G. T., and Corr, C. F., J. Biol. Chem., 116, 119, 129 (1936).

16. Kalckar, H. M., Biochem. J., 33, 631 (1939).

17. Pillai, R. K., Biochem. J., 32, 1087 (1938).

18. Parnas, J. K., Handbuch der Enzymologie, II, 902 (Leipzig, 1940).

19. Needhair, J., Shen, S. C., Needham, D. M., and Lawrence, H. S. C., Nature, 147, 766 (1941).

20. Geiger, A., Biochem. J., 34, 465 (1940).

21. ОсноА, S., J. Biol. Chem., 141, 245 (1941). 


\title{
Discussion on Phosphorylation
}

\author{
H. M. KALCKAR \\ Washington University, St. Louis, Chairman
}

Dr. Kalckar:

It seems advisable to begin this discussion on phosphorylation with a brief survey of the major present-day problems in this field. The mechanism of the compulsory coupling between oxidation of triose and phosphorylation of adenosine nucleotides was solved when Warburg and Christian isolated and crystallized the catalyst of the phosphotriose oxidation and Negelein and Brömel isolated the 1,3-diphosphoglyceric acid. Warburg and his collaborators also demonstrated the transfer of the phosphate from the carboxyl group to adenosinediphosphate. Both the oxidation process (1) and the phosphate transfer are easily reversed reactions:

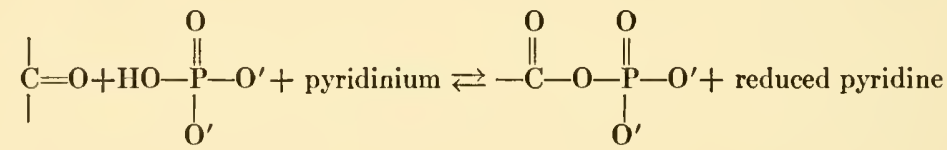

(2)

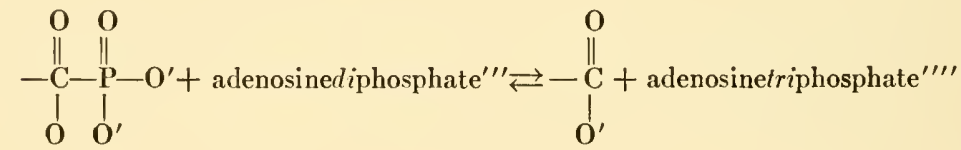

These two reversible reactions explain completely the findings of Needham, Meyerhof, and others that the utilization of the labile phosphate in adenosinetriphosphate determines the extent of oxidation of phosphotriose.

Lipmann's observations of the formation of acetylphosphate in the bacterial pyruvate oxidation furnished another important example of carbonyl oxidation coupled with phosphate uptake.

Carbonyl oxidation is not the only type of oxidation that is coupled with phosphorylation. Oxidations of fumaric acid or even of succinic acid are coupled with phosphorylations, but the mechanism of these reactions has not been clarified.

It has been mentioned that the extent of triose oxidation was automatically regulated by the removal of labile phosphate in adenosine- 
triphosphate and it is therefore of interest to discuss briefly the problems relating to adenylpyrophosphate utilization. The utilization of adenylpyrophosphate takes place by easily reversed reactions or by irreversible degradation. The phosphorylation of carboxylate or amidine ions represents easily reversed dephosphorylations of adenylpyrophosphate. The phosphorylations of the hydroxy groups of monohexoses or glycerol and the mineralization of phosphate represent the irreversible degradations of adenylpyrophosphate. These irreversible strongly exergonic reactions are probably essential in the energy transformations.

The phosphorylation of glucose by adenylpyrophosphate has been observed by Euler and Adler in brewer's yeast, and by Meyerhof in baker's yeast and in extracts of animal tissue. Strangely enough, the enzyme called hexokinase does not occur in muscle extracts. This may be related to the fact that the concentration of free glucose inside the muscle cell is very low. Perhaps phosphorylation of glucose in the skeletal muscles takes place in the so-called cell membrane and only there. I think that there is every reason to look for the hexokinase enzyme in the water-insoluble residue. Recently Colowick and I found that phosphorylation of glucose by adenylpyrophosphate when adenosinediphosphate is the phosphate donor needed, besides hexokinase, another protein which occurred only in skeletal muscle and was therefore called myokinase. Myokinase is active in amounts smaller than one microgram per milliliter and is extremely resistant to acid treatment. I have found recently that myokinase is also necessary for the dephosphorylation of adenosinediphosphate in muscle tissue. The experiments were carried out with suspensions of myosin as described by Engelhardt and Ljubimova.

The exact function of myokinase is not known. Dr. Johnson has suggested that myokinase catalyzes a transfer of phosphate from one molecule of adenosinediphosphate to another molecule, thus forming from two moles of adenosinediphosphate one mole of adenosinetriphosphate and one mole of adenosinemonophosphate. One might also consider the formation of a dinucleotide between adenosinetriphosphate and adenylic acid of the type that has been isolated by Kiessling and Meyerhof. Myokinase might function not only as a catalyst but also as a phosphate transfer system. The inhibition of myokinase by adenylic acid may also indicate a reversed reaction of myokinase with adenosinediphosphate.

Adenylpyrophosphate phosphorylates glucose or fructose to 
hexose-6-phosphate. Sutherland, Colowick, and Cori have shown recently that this ester can be converted to hexose-1-phosphate and thus to starch or glycogen.

The phosphorylation of hexosemonophosphate to hexosediphosphate has been observed in several tissues and also in muscle extracts. This reaction is probably strongly exergonic, like the hexokinase reaction.

Meyerhof and Lohmann have shown that the dephosphorylation of adenylpyrophosphate to adenosinedi- or monophosphate and orthophosphate is one of the strongest exergonic reactions in biological systems. The enzyme is present in large amounts in muscle extracts but the myosin fraction contains also large amounts of this enzyme, and Engelhardt and Ljubimova have found that even highly purified myosin contains adenosinetriphosphatase.

The utilization of adenylpyrophosphate by these irreversible reactions probably determines the extent of numerous important oxidations. The great question is how the irreversible utilization of adenylpyrophosphate is regulated. The existence of adenylpyrophosphate in resting muscles with very low metabolism clearly shows that the enzymes which catalyze the breakdown of pyrophosphates must be in a more or less inactivated state and are fully activated only under certain conditions. The state of structural proteins such as myosin might determine the extent of the activation of the enzymes which catalyze the degradations of adenylpyrophosphate. Discharged structural proteins might even be enabled to use the large amount of energy liberated by pyrophosphate degradations for recharging by participating in the transfer of phosphate.

We do not yet know how irreversible enzymatic reactions are regulated or how they are directed in order to transform chemical energy into mechanical energy. It has become clear, however, that the irreversible dephosphorylations of adenylpyrophosphate represent some of the most important degradations in biological systems.

\section{Otto Meyerhof, University of Pennsylvania:}

May I discuss three of the points that have been proposed by Dr. Kalckar:

1. Because of the specific structure of the living cell, the metabolism can be regulated differently from the metabolic processes in extracts or other preparations of dead cells. At least one such difference can easily be explained by the insufficient stability of the extracted enzyme. I refer to the accumulation of hexosediphosphate in yeast preparations in contrast to the balance of formation and 
decomposition of this ester in living yeast. Indeed the occurrence of the same phosphorylated intermediaries in living cells as in extracts is clearly demonstrated by the experiments of Miss Macfarlane, Werkman, Dische, and others. But the adenylpyrophosphatase, which is responsible for the fermentation of hexosediphosphate in the absence of a stoichiometric amount of phosphate acceptors, is weakened even by drying the yeast, more by incubation and extraction of the dried yeast, and it can be completely destroyed by precipitation with acetone.

On the other hand, we may assume that in the living cell excess phosphorylation of sugar occurs in connection with growth. The starting point of these syntheses may be some other ester instead of hexosediphosphate; nevertheless the autocatalytical increase of this ester in a fermenting yeast extract can be taken as a good model for material growth brought about by the energy of fermentation; indeed, such an extract, containing sugar, will not start fermentation unless it is "inoculated" by a trace of hexosediphosphate, which then "grows" at the expense of sugar.

2. At least some of the experiments of different authors quoted by Dr. Lipmann seem not too reliable in regard to abnormally high oxidation quotients. I refer especially to cases with $\mathrm{Qo}_{2}$ values of 0 to 1 . Under such conditions the manometric method is not accurate enough, and a completely stationary state during the time of the experiment is not assured.

Furthermore, it seems to me, the distribution of oxidized to reduced cozymase in toto cannot be used as the basis for deciding how far the hydrogen transfer by means of cozymase is affected by respiration. This transfer occurs by means of the bound cozymase of specific enzymes, and the oxidative state of such a compound may well be altered by the oxygen transfer from oxidative catalysts without an appreciable change in the overall distribution of oxidized to reduced cozymase.

3. The interesting finding of Dr. Kalckar that the action of hexokinase of yeast is supplemented by a heat-stable enzyme of muscle, "myokinase," by which adenosinediphosphate transfers its labile phosphate group to glucose, points to a difference, already well known, between the enzymes in muscle and yeast preparations. While the former (extract, acetone powder, etc.) retains the ability to use adenylic acid as the phosphorylating coenzyme even after long dialysis and "ageing," in yeast preparations the reaction step,

(2) P-acceptor + adenosinediphosphate $\leftrightarrows \mathrm{P}$-acceptor - phosphate + adenylic acid, 
is easily destroyed, and only the first reaction step of transphosphorylation,

(1) P-acceptor + adenosinetriphosphate $\leftrightarrows \mathrm{P}$-acceptor - phosphate + adenosinediphosphate

is still occurring. A more detailed study of the different enzyme proteins in these phosphorylating reactions along the lines suggested by Colowick and Kalckar seems highly desirable.

\section{J. Johnson, University of Wisconsin:}

The present status of our knowledge on biological phosphorylations raises the question whether energy of phosphorylation is the sole form in which energy from food oxidation (or fermentation) is made available for metabolic reactions. In other words, is phosphorylation the only device employed by the cell to utilize oxidative energy?

The only answer that can be made at present is that phosphorylation is the only mechanism we know of by which a part of the energy derivable from the burning of food material can be made available for endergonic life processes. There appears to be no $a$ priori reason to suspect the existence of another mechanism. In fact, the simplicity of a single-mechanism hypothesis seems very attractive. On the other hand, we have no reason to doubt the existence of other "energy-fixing" reactions. The question is entirely open.

There is, however, at least one fragment of evidence that definitely favors the single-mechanism hypothesis. Yeast growing anaerobically in a simple medium must derive all the energy for its growth reactions from the conversion of glucose into ethyl alcohol and carbon dioxide. Unless our present views of the mechanism of this conversion are erroneous, no energy-fixing mechanism other than phosphorylation exists in alcoholic fermentation. Therefore the only form in which energy from the fermentation process is available to the organism is energy of phosphorylation. It follows, of course, that the yeast cell is capable of utilizing, directly or indirectly, energy of phosphorylation for all its metabolic energy requirements. Hence any other energy-fixing reactions which might be postulated to occur during aerobic sugar breakdown are at least not essential for yeast growth. 


\section{Fritz Lipmann, Massachusetts General Hospital:}

On several occasions during this symposium acetylphosphate has been mentioned as an intermediate in carbohydrate breakdown. Since I have not yet published a complete account of my experiments, I should like to take this opportunity to summarize the evidence so far accumulated for the formation of acetylphosphate as an intermediate in pyruvic acid oxidation. Early in my study of the oxidation of pyruvic acid in lactic acid bacteria it was observed that inorganic phosphate was an integral part of the pyruvic acid oxidation system (1). In partial explanation of its necessity it was shown that with oxidation of pyruvic acid, phosphate could be transferred to adenylic acid to form adenosinepolyphosphate (2). In other words, pyruvic acid oxidation generated energy-rich phosphate bonds (terminology of ref. 3). In analogy to Negelein and Brömel's phosphoglycerylphosphate (4), acetylphosphate was then suspected to be the phosphorylated intermediate between pyruvate and adenosine polyphosphate. In confirmation it was found that synthetical acetylphosphate enzymatically transferred phosphate to adenylic acid with the formation of adenosinetriphosphate (5). This observation led to a reinvestigation of the phosphate turnover in pyruvic acid oxidation. In the bacterial metabolism pyruvate is fortunately not oxidized farther than the acetate stage. Under favorable conditions, therefore, one could hope to demonstrate the accumulation of a phosphorylated precursor of acetic acid.

Earlier attempts had always failed to disclose any disappearance of inorganic phosphate during the oxidation of pyruvate. Now that acetylphosphate was suspected as intermediate, its stability was studied, and it was found that its formation would have been overlooked because in the course of all the known procedures of phosphate assay it would have been decomposed to inorganic phosphate. Therefore the problem arose of finding a method for the determination of inorganic phosphate in the presence of a compound of the stability of acetylphosphate, since this compound does not withstand the alkalinity of the magnesia mixture tolerated by creatinephosphate, nor the acidity of the molybdate reagent. It appeared possible, however, to completely precipitate inorganic phosphate as calcium phosphate in dilute alcohol at a $\mathrm{pH}$ of 8 , where acetylphosphate is stable. The use of this more delicate precipitation procedure showed indeed that large amounts of inorganic phosphate disappeared during pyruvate oxidation with or without fluoride (6). Phosphorylation, oxygen consumption, and pyruvate disappearance 
occurred in definite proportions and in good agreement with the equation:

$$
\begin{gathered}
\mathrm{CH}_{3} \cdot \mathrm{CO} \cdot \mathrm{COOH}+\mathrm{HO} \cdot \mathrm{PO}_{3} \mathrm{H}_{2}+\mathrm{O}=\mathrm{CH}_{3} \cdot \mathrm{CO} \cdot \\
\mathrm{OPO}_{3} \mathrm{H}_{2}+\mathrm{CO}_{2}+\mathrm{H}_{2} \mathrm{O}
\end{gathered}
$$

Recently the formation of a labile phosphate compound could be demonstrated directly by the use of the method with which creatinephosphate was originally discovered both by Fiske and Subbarow and by the Eggletons. This method consists in reading the development of the blue color against a standard of inorganic phosphate, the increase in color with time being proportional to the breakdown of the labile phosphate compound. The half-decomposition time of our phosphorylation product (and of acetylphosphate) is about one minute at room temperature. This rapid decomposition of the natural as well as the synthetic product is due not merely to the acidity of the solution but largely to a catalytic effect of molybdate. Recently silver fractions containing the labile phosphate have been obtained from trichloroacetic acid extracts. Although, as should be emphasized, pure preparations of the phosphorylation product have not yet been obtained, "the reported results may be taken as fair evidence that the intermediate formed during pyruvic acid oxidation is in fact acetylphosphate.

\section{REFERENCES}

1. Lipmann, F., Enzymologia, 4, 65 (1937).

2. Lipmann, F., Nature, 143, 281 (1939).

Оснод, S., J. Biol. Chem., 138, 751 (1941).

3. Lipmann, F., Advances in Enzymology, 1, 99 (1941).

4. Negelein, E., and Brömel, H., Biochem. Z., 300, 225 (1939).

5. Lipmann, F., Nature, 144, 381 (1939).

6. Lipmann, F., J. Biol. Chem., 134, 463 (1940).

- Since submitting this paper we have succeeded in isolating silver acetylphosphate from the impure silver fractions. 


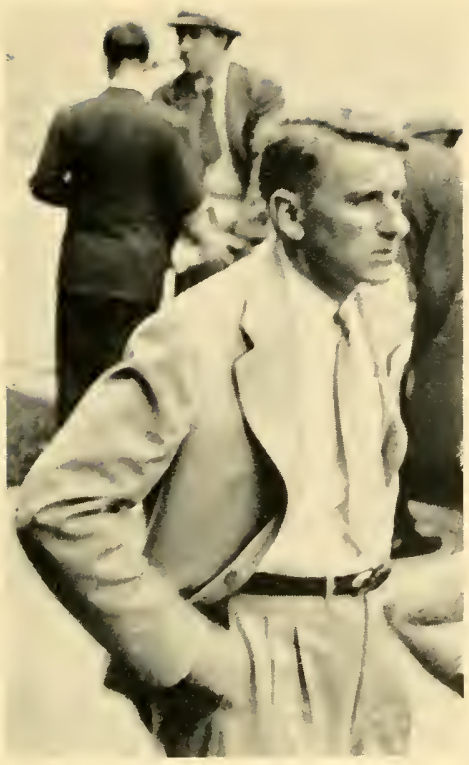

Schlenk

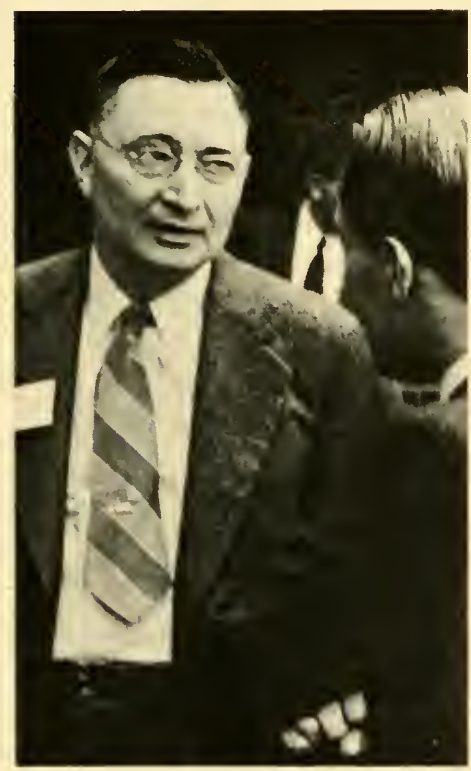

WeRkMaN

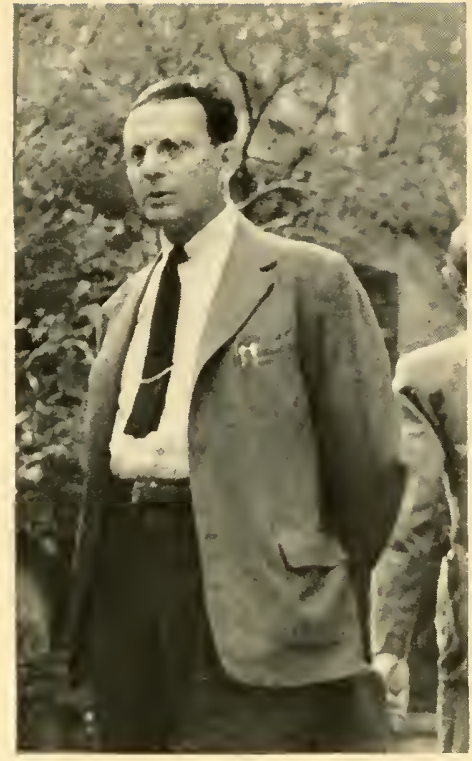

Stern

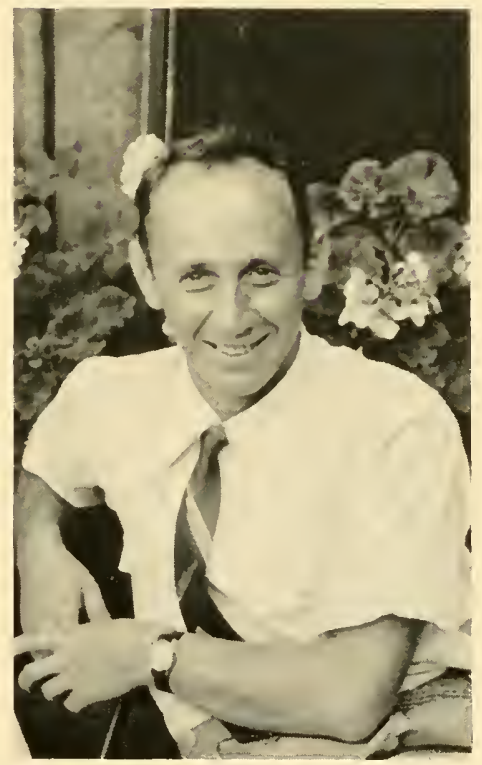

HAAS

CLOSE-UPS OF FOUR PARTICIPANTS 



\title{
Metabolic Cycles and Decarboxylation
}

\author{
E. A. EVANS, JR. \\ University of Chicago ${ }^{*}$
}

TF I WERE to adhere rigidly to a discussion of the topic assigned 1 me I should be compelled to mention almost every aspect of our present knowledge of intermediary metabolism. I am claiming, therefore, the traditional privilege of discussing those matters that seem of particular interest and importance.

From what we know of the chemical constitution of most cells, it is to be expected that those interactions of cell constituents by which the organism obtains the energy necessary for continued existence should exhibit certain characteristics of continuity and recurrence, as does the cell itself. The utilization of foodstuffs by the cell frequently involves a cyclic chain of chemical transformations in which certain cell constituents, usually present in small and apparently constant amounts, facilitate the transformation of larger quantities of other metabolites in reactions releasing energy or leading to the formation of the actual protoplasmic fabric of the cell itself. The synthesis of urea, the transformation of glycogen into lactic acid, and, in a broader sense, the transport of the respiratory gases by the blood are familiar and typical examples of the cyclic mechanisms by which the organism maintains the balance necessary for its existence in the midst of the dynamic processes by which it functions.

In the past few years such a cyclic series of reactions has been proposed for the mechanism of oxidation of carbohydrate in various tissues-more specifically for the oxidation of pyruvic acid in voluntary musculature. This scheme, the so-called citric acid cycle, was proposed by Krebs $(1,2,3,4)$. In its original form the theory was concerned with the oxidation of pyruvic acid by minced pigeon breast muscle, and although the generalizations of the theory have been extended in part to other tissues and species $(5,6)$, the most convincing and complete data are those derived from suspensions of muscle tissue.

- The original work reported in this paper was aided in part by grants from the John and Mary R. Markle Foundation and from the Dr. Wallace C. and Clara A. Abbott Memorial Fund of the University of Chicago. 
A great variety of chemical transformations involving pyruvic acid has been demonstrated in different species of living cells, isolated enzyme systems, etc. (representative reactions are listed in Table 1 which is not, however, complete). In some cases several of these diverse ways of treating pyruvic acid can be demonstrated to occur in the same cell. In such circumstances there is considerable advantage in a working hypothesis in which reactions involving the same compound are regarded as components of an integrated system. In a tissue such as muscle, where specialization of function might be expected to reflect a similar specialization of metabolism, an hypothesis of this type should be especially fruitful. The citric acid cycle represents, then, an attempt to summarize the available information with respect to the metabolism of pyruvic acid in pigeon breast muscle. Such a summary is of value only so long as it does not conflict with experimental observations and to the extent that it adequately represents the experimental foundation on which it rests. At the moment there are no observations that can be regarded as invalidating the theory, although objections to one or another feature of the scheme have been made. At the risk of adding little that is new to what you already know, I should like to discuss several aspects of the citric acid theory, particularly in regard to its experimental basis. The cycle itself is shown diagrammatically in Figure 1.

The essential experimental support for the theory can be summarized in the following equations:

In the presence of malonate:

(1) Fumarate + pyruvate $+2 \mathrm{O}_{2} \rightarrow$ succinate $+3 \mathrm{CO}_{2}+\mathrm{H}_{2} \mathrm{O}$

(2) Malate + pyruvate $+2 \mathrm{O}_{2} \rightarrow$ succinate $+3 \mathrm{CO}_{2}+2 \mathrm{H}_{2} \mathrm{O}$

(3) Oxalacetate + pyruvate $+1 \frac{1 \%}{2} \mathrm{O}_{2} \rightarrow$ succinate $+3 \mathrm{CO}_{2}+\mathrm{H}_{2} \mathrm{O}$

(4) Citrate $+\mathrm{O}_{2} \rightarrow$ succinate $+2 \mathrm{CO}_{2}+\mathrm{H}_{2} \mathrm{O}$

(5) $\alpha$-Ketoglutarate $+1 / 2 \mathrm{O}_{2} \rightarrow$ succinate $+\mathrm{CO}_{2}+\mathrm{H}_{2} \mathrm{O}$

In the absence of malonate:

Succinate $+\frac{12}{12} \mathrm{O}_{2} \rightarrow$ fumarate $+\mathrm{H}_{2} \mathrm{O}$

Pyruvic acid $+2 \frac{1 / 2}{12} \mathrm{O}_{2} \rightarrow 3 \mathrm{CO}_{2}+\mathrm{H}_{2} \mathrm{O}$ (Equations $1+6$ )

In nitrogen:

2 Oxalacetate + pyruvate $\rightarrow$ citrate $+\mathrm{CO}_{2}+$ malate

Oxalacetate + citrate $\rightarrow \alpha$-ketoglutarate $+\mathrm{CO}_{2}+$ malate.

The demonstration, in suspensions of muscle tissue, of the stoichiometric relationships expressed in these equations constitutes the experimental proof on which the citric acid cycle is based. This demonstration has two aspects-one concerned with the qualitative presence of these reactions, i.e., with the existence of the necessary 
Table 1.-Reactions of pyruvic acid (P.A.)

P.A. $\rightarrow$ acetaldehyde $+\mathrm{CO}_{2}$

P.A. $+\frac{1}{2} \mathrm{O}_{2} \rightarrow$ acetic acid $+\mathrm{CO}_{2}$

2P.A. $+\mathrm{H}_{2} \mathrm{O} \rightarrow$ lactic acid + acetic acid $+\mathrm{CO}_{2}$

P.A. $+2 \mathrm{H} \rightarrow$ lactic acid

P.A. + oxalacetic acid $\rightarrow$ citric acid

2P.A. $\rightarrow$ acetic acid + formic acid

P.A. + glutamic acid $\rightarrow$ alanine $+\alpha$-ketoglutaric acid

QP.A. $\rightarrow$ acetoacetic acid

2 P.A. $\rightarrow \beta$-hydroxybutyric acid

P.A. $\rightarrow$ alanine

P.A. + acetate $\rightarrow$ acetopyruvate $\rightarrow$ acetoacetate

P.A. $+\mathrm{CO}_{2} \rightarrow \alpha$-ketoglutarate

2P.A. $\rightarrow$ acetylmethylcarbinol yeast

brain, gonococcus

gonococcus, brain, liver

muscle, tumor

muscle, liver, kidney

streptococcus

muscle

liver

muscle

muscle

liver

liver

muscle

enzymic channels through which these reactions may flow, and the other with their quantitative capacity to perform the task imposed upon them by their postulated role in the cycle. To the extent that a suspension of minced pigeon breast muscle retains the chemical reactions involved in its respiration in the intact state, the reactions summarized in equation 7 may be regarded as most closely approxi-

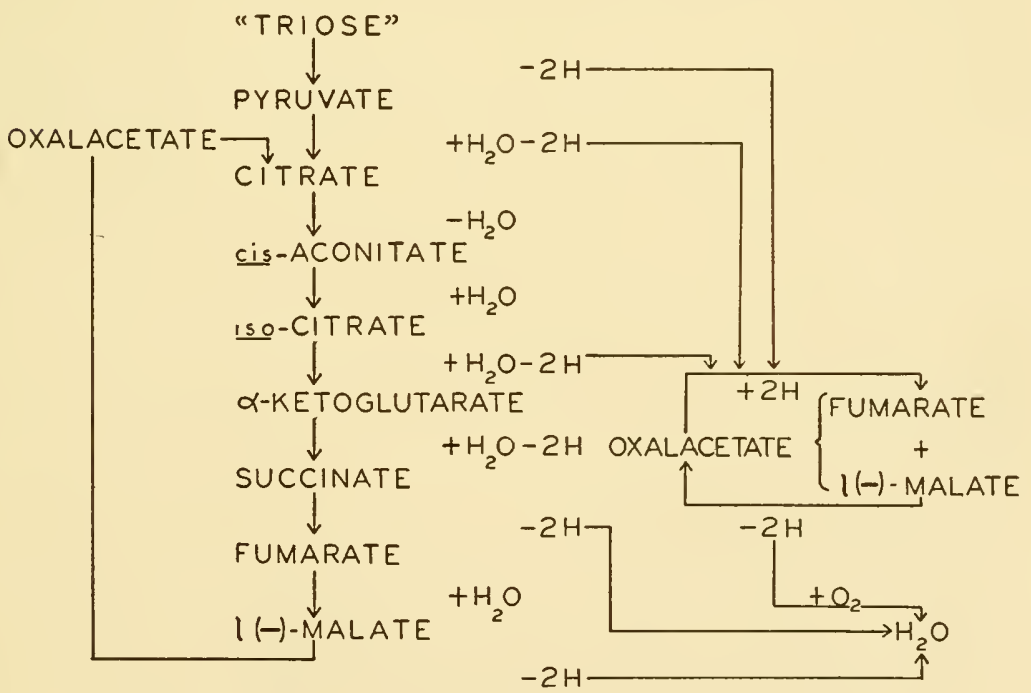

Figure 1.-Scheme of the oxidative breakdown of carbohydrate in pigeon breast muscle 
mating the physiological state, since they occur when pyruvate is added to the tissue in the presence of an adequate supply of oxygen and in a medium approximately physiological in ionic concentration and $\mathrm{pH}$. In these circumstances the stoichiometric relationships of equation 7 are very closely realized, and it seems certain that the total oxygen uptake of the tissue is utilized for the oxidation of pyruvic acid completely to carbon dioxide and water. Pyruvic acid will also disappear in the absence of oxygen. The quantity concerned is about one-tenth that removed aerobically, and although lactic acid, acetic acid, carbon dioxide, succinic acid, and betahydroxybutyric acid have been recognized as products of this anaerobic reaction, the details are still obscure.

Equations 1 to 5 deal with reactions carried out in the presence of malonic acid. Equation 1 is the most important reaction of the theory, since it demonstrates unequivocally an oxidative formation of succinic acid from fumaric acid. In the presence of proper concentrations of malonic acid the oxidation of pyruvate by pigeon breast muscle can be almost entirely inhibited. When fumarate is added, however, pyruvate is oxidized and in accordance with equation 1 we find, per mole of fumarate added, one mole of pyruvate and 2 moles of oxygen consumed, and 1 mole of succinate and 3 moles of carbon dioxide formed. A direct reduction of fumarate to succinate under these circumstances is inhibited by the malonic acid present, as can be shown by anaerobic experiments; that is, we find much more succinate formed from fumarate in the malonatepoisoned muscle in oxygen than in nitrogen. Since it is impossible to explain the conversion of fumarate to succinate in the presence of malonate by anaerobic reduction, a second mechanism must exist which is oxidative and unaffected by malonate and which results in the transformation of fumarate into succinate. It should be emphasized that we find in this transformation the stoichiometric relationships of equation 1 . The citric acid cycle postulates that this oxidative transformation involves the intermediate formation of citric acid. Regardless of the nature of the intermediates, however, any explanation of the oxidation of pyruvic acid by muscle must account for reaction 1, i.e., what might be termed the Krebs reaction.

The demonstration of the stoichiometric relationships of equation 1 depends upon the recognition of essential experimental conditions. In view of the fundamental nature of this reaction it may be permissible to discuss a few matters of experimental detail.

The working plan of the experiments summarized in equations 1 
to 5 involves interruption of the cycle at the succinate-fumarate stage by poisoning with malonic acid. Implicit here is the assumption that malonate acts specifically on this particular reaction, i.e., the oxidation of succinate to fumarate. The inhibition of succinic dehydrogenase by malonic acid in isolated enzyme preparations has been recognized and demonstrated by various investigators $(7,8)$. The inhibition is competitive in nature; that is, it depends not upon the absolute concentration of malonate but on the relative quantities of succinate and malonate. Since the basis of this competitive inhibition is the resemblance in chemical structure between succinic and malonic acids, it follows that malonate will probably inhibit the enzymic transformation of any substrate bearing some chemical resemblance to its own structure. However, the available data suggest that the effect of the poison is most pronounced with the succinic dehydrogenase. Expressed quantitatively, with 1:10 suspensions of pigeon breast muscle in calcium-free phosphate saline, 0.001 M malonate inhibits pyruvate utilization about 20 per cent. Higher concentrations of malonate, around $0.025 \mathrm{M}$, inhibit to more than 90 per cent.

As equation 1 indicates, fumaric acid will abolish the inhibitory effect of malonate in muscle tissue with the simultaneous utilization of one mole of pyruvate and 2 moles of oxygen to give 1 mole of succinate and 3 moles of carbon dioxide. The extent of this fumarate effect, however, depends upon the relative concentrations of malonate and fumarate. Krebs, in a long series of experiments (2), has shown very clearly that the demonstration of the stoichiometric relationships of equation 1 depends upon the presence of an adequate concentration of malonic acid. When the malonate concentration is too low, as, for example, with $0.001 \mathrm{M}$ malonate in the presence of $0.0025 \mathrm{M}$ fumarate, the gradual conversion of fumaric acid to succinic acid will so increase the succinic acid concentration as compared with that of the malonate that succinic acid will be oxidized to fumaric acid at a rate sufficient to provide for the continuation of the cycle at the full rate. On the other hand, with $0.025 \mathrm{M}$ malonate the stoichiometric relationships of equation 1 are realized. It is obvious, then, that the relationships expressed in equations 1 to 5 can be demonstrated only in the presence of adequate concentrations of malonic acid. When this is done, the experimental data are in fairly close approximation to the expected values.

In similar experiments with pig heart muscle, Smyth (5) has observed an occasional failure with citric acid to obtain the quantities 
involved in reaction 4 . He offers several possible explanations for these occasional failures, although the matter must still be considered unsettled.

In terms of the postulated cycle, the possible participation of intermediate steps such as those listed in equations 1 to 5 can also be critically examined from the standpoint of the minimum rates at which they occur. This has already been discussed in considerable detail elsewhere (9). If we assume that the citric acid cycle represents the entire respiratory process involved in the oxidation of pyruvic acid in pigeon breast muscle, then the total oxygen uptake of the tissue represents a total of five consecutive reactions of the cycle. Under circumstances in which individual reactions of the cycle are isolated-for example, when alpha-ketoglutarate is added to the malonate-poisoned tissue-the rate of oxygen uptake for the conversion of alpha-ketoglutarate to succinate cannot be less than one-fifth that for pyruvate oxidation. If the reaction velocity is below this minimum requirement, the step can be excluded as an intermediate in the overall reaction. On the other hand, if the isolated reaction proceeds with a velocity greater than the minimum rate, it may still be considered as an intermediate, since in the intact system the concentration of the particular enzyme concerned may exceed the quantity of the substrate normally available. When the reactions summarized in equations 1 to 5 are examined from this viewpoint, we find in each case that the rate of the isolated step proceeds with the necessary velocity consonant with its composing part of the cycle.

The existence of what I have termed the Krebs reaction is generally accepted: most critical comment on the citric acid hypothesis centers around the postulated intermediate formation of citric acid. It has been argued that failure of this substance to accumulate in large amounts during pyruvate oxidation in various tissues is contrary to its postulated intermediate role. However, the accumulation of an intermediate must represent a balance between its synthesis and its removal, and the accumulation of large amounts of citric acid would be much more difficult to reconcile with the premise that it is an active intermediate substance than would the fact that it accumulates to only a limited extent. In addition, Krebs has demonstrated an anaerobic reaction between citrate and oxalacetate to yield alpha-ketoglutarate and malate (equation 9). Although there is reason to question the validity of such anaerobic experiments as the basis for assigning the role of hydrogen carriers to the oxalacetate- 
malate system in the aerobic cycle, this reaction may also explain why citrate does not accumulate to any extent. On the other hand, the only reason we have for including citric acid in the cycle is the rapidity of its formation from oxalacetate and pyruvate as indicated in equation 8 and the fact that its oxidative breakdown will yield alpha-ketoglutarate and succinate at the required rate. It has been suggested, first by Breusch (10) and later by others, that citrate formation represents a side reaction in pyruvate metabolism-a means for disposing of excessive amounts of the dicarboxylic acids. There is, however, no experimental support for this concept at the present time; the simpler hypothesis, in which citric acid is regarded as an intermediate in the cycle, is probably to be preferred as a basis for further experiments. It should be emphasized that succinate is formed in malonate-poisoned muscle at the proper rate only by those compounds listed as intermediates, although a great number of other possible substances have been examined for their ability to do this. It seems improbable, therefore, that the quantitative formation of succinate from citrate in the malonate-poisoned muscle is without significance for the role of citrate in muscle metabolism; Smyth's failure to obtain consistent results with citrate in pig heart muscle may be due to our ignorance of the essential conditions for citrate oxidation, as he suggests.

With other tissues the evidence for similar mechanisms of pyruvate oxidation is much less complete. It has been shown in pigeon liver, for example, that the enzyme systems necessary for the reactions of the citric acid cycle are present (6). Whether or not they play any considerable role in its metabolic function remains a question for further study. We do have evidence that alphaketoglutaric acid formation can occur in this tissue independently of the reactions of the citric acid cycle $(9,11,12)$.

Demonstration of the synthesis of alpha-ketoglutaric acid from pyruvic acid in pigeon liver has been followed by efforts to ascertain the mechanism involved. The reaction proceeds in the presence of malonic acid and without the addition of any of the four-carbon dicarboxylic acids, presumably by a direct utilization of the threecarbon compound. The scheme of Toeniessen and Brinkmann (13) for succinate synthesis from pyruvate in muscle, involving the intermediate formation of diketo-adipic acid, could be disregarded, since pigeon liver is incapable of metabolizing formic acid at a rate necessary for this mechanism to operate (6).

Since pigeon liver can form citric acid from oxalacetic acid and 
can also oxidize citric acid to alpha-ketoglutaric acid, the reaction of Wood and Werkman involving the carboxylation of pyruvic acid to yield oxalacetate (14) offers an attractive hypothesis for alpha-ketoglutarate synthesis, i.e., oxalacetate + pyruvate $\rightarrow$ citrate $\rightarrow$ alpha-ketoglutarate. Slotin and I carried out experiments to test this hypothesis, using carbon dioxide containing radioactive $\mathrm{C}^{11}$ and found that the succinic acid formed on the addition of pyruvate to malonate-poisoned pigeon liver, presumably through the intermediate formation of alpha-ketoglutaric acid, was devoid of radioactivity. However, when we extended these experiments to the isolation of alpha-ketoglutaric acid as the dinitrophenylhydrazone, we could clearly observe the utilization of carbon dioxide in the formation of radioactive alpha-ketoglutarate (15). As the earlier succinate experiments suggested, and as was confirmed later by direct examination of the isolated keto acid, the radioactivity is confined entirely to the carboxyl group of this compound.

The fact that carbon dioxide is used in the synthesis of alphaketoglutarate from pyruvate in pigeon liver and that the assimilated carbon dioxide is present entirely in the carboxyl group alpha to the carbonyl oxygen, is now firmly established in view of the identical results of Wood, Werkman, Hemingway, and Nier (12), using the stable $\mathrm{C}^{13}$, and of Slotin and me, with the short-lived radioactive $\mathrm{C}^{11}(11,15)$. These data cannot be reconciled with the intermediate formation of citric acid, since any symmetrical intermediate molecule of this type would yield alpha-ketoglutarate with radioactivity at both carboxyls. Therefore the original conception of alpha-ketoglutarate synthesis occurring by way of pyruvate and carbon dioxide condensation to oxalacetate, and the subsequent reaction of this dicarboxylic acid with another mole of pyruvate to yield citric acid, must be abandoned. Support for such a view is also derived from experiments in which non-radioactive citrate was added to liver suspension in radioactive bicarbonate medium during the synthesis of alpha-ketoglutaric acid. Table 2 lists experiments showing that the addition of $25 \mathrm{mg}$. of sodium citrate affects neither the yield of alpha-ketoglutaric acid nor the ratio of activity per mg. carbon of the alpha-ketoglutarate to that of the medium. If citrate is an intermediate in the formation of alphaketoglutaric acid from pyruvate and carbon dioxide, the addition of non-radioactive citrate to the synthesizing tissue should yield alpha-ketoglutaric acid in which the radioactivity had been considerably diluted. Failure to demonstrate such an effect may be 
considered additional evidence for the nonparticipation of citric acid in the synthesis of alpha-ketoglutaric acid from pyruvate and carbon dioxide.

Table 2.-The effect of citrate on the radioactivity of synthesized alpha-ketoglutaric acid ${ }^{*}$

\begin{tabular}{cccc}
\hline Experiment & $\begin{array}{c}\text { Sodium } \\
\text { citrate added }\end{array}$ & $\begin{array}{c}\alpha \text {-Ketoglutarate } \\
\text { synthesized }\end{array}$ & $\begin{array}{c}\text { Activity of } \\
\alpha \text {-ketoglutarate }\end{array}$ \\
\hline \multirow{2}{*}{1} & mg. & mg. & per mg. C \\
2 & 0 & 31.4 & 0.148 \\
& 25 & 28.8 & 0.113 \\
& 0 & 36.9 & 0.116 \\
25 & 39.8 & 0.116
\end{tabular}

* Experimental conditions were similar to those in the experiments reported in Table 3 . The sodium citrate was added simultaneously with the pyruvic acid.

Wood and Werkman and their collaborators (12) have suggested that isocitric acid rather than citrate serves as an intermediate, such a scheme retaining in skeleton form the original suggestion that alpha-ketoglutarate was produced by way of the citric acid cycle. In view of the demonstrated equilibrium, in most tissues, between citrate and isocitrate (16), additional evidence is required to establish this hypothesis. The exclusion of citric acid as an intermediate in alpha-ketoglutarate synthesis might well lead to an examination of whether oxalacetate itself is formed as the primary product of carbon dioxide assimilation in pigeon liver. The great chemical reactivity and instability of this compound make it improbable that it should accumulate to any appreciable extent under our experimental conditions. Though we have studied a wide variety of conditions, we have failed to obtain any evidence that the compound was present during the course of alpha-ketoglutarate synthesis; or, to express the matter more precisely in experimental terms, we have failed to observe the accumulation of any substance that would release radioactive carbon dioxide on treatment with aniline citrate. Certainly it would be desirable to have more convincing evidence that oxalacetic acid is the primary product of carbon dioxide assimilation in pigeon liver.

There seems little doubt that the utilization of carbon dioxide.by pigeon liver is a process of considerable magnitude. Table 3 gives data from a series of typical experiments which indicate that as much as 5 per cent of the original activity of the added inorganic 
carbonate appears in the synthesized alpha-ketoglutaric acid. Unfortunately these data are difficult to interpret in terms of any precise mechanism of alpha-ketoglutarate formation. Since all the

Table 3.-Synthesis of alpha-ketoglutarate in radioactive bicarbonate medium*

\begin{tabular}{|c|c|c|c|c|c|c|c|}
\hline \multirow[t]{2}{*}{$\operatorname{Exp}$} & \multirow{2}{*}{$\begin{array}{l}\alpha \text {-Keto- } \\
\text { glutarate } \\
\text { synthe- } \\
\text { sized }\end{array}$} & $\begin{array}{r}\text { Activ } \\
\alpha \text {-ketog }\end{array}$ & $\begin{array}{l}\text { ty } \dagger \text { of } \\
\text { lutarate }\end{array}$ & \multirow{2}{*}{$\begin{array}{c}\text { Total } \\
\text { activity } \\
\text { of } \\
\text { medium }\end{array}$} & \multirow{2}{*}{$\begin{array}{l}\text { Terminal } \\
\text { activity } \\
\text { of medium } \\
\text { per mg. } \\
\text { inorganic } \\
\text { carbon }\end{array}$} & \multirow{2}{*}{$\begin{array}{c}\text { Ratio } \\
\begin{array}{c}\alpha \text {-ketoglutarate } \\
\text { activity }\end{array} \\
\begin{array}{c}\text { original activ- } \\
\text { ity of medium }\end{array}\end{array}$} & \multirow{2}{*}{$\begin{array}{c}\text { Ratio } \\
\text { medium activ- } \\
\text { ity per mg. C } \\
\alpha \text {-ketoglutarate } \\
\text { per mg. C }\end{array}$} \\
\hline & & Total & $\begin{array}{c}\text { per } \\
\text { mg. C }\end{array}$ & & & & \\
\hline & $\mathrm{mg}$. & & & & & & \\
\hline 1 & 31.4 & 0.471 & 0.036 & 8.74 & 0.078 & 0.054 & 2.0 \\
\hline 2 & 27.5 & 4.09 & 0.362 & 67.0 & 1.13 & 0.061 & 3.1 \\
\hline 3 & 55.0 & 3.85 & $\begin{array}{l}0.171 \\
0.161 \ddagger \\
0.178\end{array}$ & 79.8 & 1.17 & 0.048 & 6.9 \\
\hline
\end{tabular}

* $7.6 \mathrm{gm}$. minced pigeon liver in $50 \mathrm{ml}$. calcium-free bicarbonate saline, pH. 7.4, containing radioactive $\mathrm{C}^{11} ; 1.7 \mathrm{ml} .0 .1 \mathrm{M}$ malonate; $10 \mathrm{ml} .0 .02 \mathrm{M}$ pyruvate; malonate added directly to tissue; pyruvate added after 10 minutes; temperature, $40^{\circ} \mathrm{C}$.; gas phase, 5 per cent carbon dioxide; experimental period, 40 minutes.

$\dagger$ Activities are expressed in divisions per second (Lauritsen electroscope); and are corrected for decay so that the values are comparable.

$\ddagger$ Activities after successive recrystallizations of alpha-ketoglutarate dinitrophenylhydrazone.

radioactivity is lost on oxidation of alpha-ketoglutaric acid to succinic acid, we can definitely conclude that not more than one carbon atom of the five of alpha-ketoglutarate is derived from the medium. From the quantity of radioactivity present in the alpha-ketoglutarate we can calculate (the details of these very approximate calculations are given elsewhere [11]) that approximately one carbon atom in ten of the alpha-ketoglutarate is derived from the inorganic carbonate of the medium. Since this figure involves very approximate calculations, and is of value only in suggesting the order of magnitude of the reaction, we are inclined to believe, as a working hypothesis, that one mole of carbon dioxide from the medium is assimilated per mole of alpha-ketoglutarate synthesized.

The synthesis of alpha-ketoglutarate is by no means the limit of carbon dioxide assimilation. Under our experimental conditions we find that alpha-ketoglutaric acid represents only about 25 per cent of the radioactivity which has been assimilated, that is, activity which can no longer be released as carbon dioxide on the addition 
of strong acid. Part of this non- $\alpha$-ketoglutarate radioactivity can be released as carbon dioxide on treatment with ninhydrin at $100^{\circ} \mathrm{C}$., and with chloramine-T (Table 4). This suggests that part of the assimilated carbon dioxide has been converted into an amino acid. Beyond this, however, we know nothing of the chemical nature of the compounds concerned.

Table 4.-Effect of ninhydrin and chloramine $\mathrm{T}$ on non- $\alpha$ ketoglutarate radioactivity*

\begin{tabular}{lcccc}
\hline \hline Experiment & $\begin{array}{c}\alpha \text {-Keto- } \\
\text { glutarate } \\
\text { synthesized }\end{array}$ & $\begin{array}{c}\text { Total } \\
\text { activity } \\
\text { of } \alpha \text {-keto- } \\
\text { glutarate }\end{array}$ & $\begin{array}{c}\text { activity } \\
\text { of medium } \\
\text { after } \mathrm{CO}_{2} \\
\text { removal }\end{array}$ & $\begin{array}{c}\text { Activity of } \\
\mathrm{CO}_{2} \text { released } \\
\text { by ninhydrin by chloramine-T }\end{array} \begin{array}{c}\text { Activity of } \\
\mathrm{CO}_{2} \text { releascd }\end{array}$ \\
\hline
\end{tabular}

\begin{tabular}{|c|c|c|c|c|c|}
\hline 1 & $\begin{array}{c}\text { mg. } \\
35.0\end{array}$ & 5.18 & 31.8 & 7.8 & 一 \\
\hline 2 & 39.0 & 6.2 & 31.2 & - & 10.39 \\
\hline 3 & 53.0 & 1.2 & 4.2 & 一 & 0.78 \\
\hline 4 & 31.6 & 0.22 & 0.929 & 0.209 & - \\
\hline
\end{tabular}

* Experimental conditions are similar to those described in Table 3. The experiments with chloramine- $T$ and with ninhydrin were carried out on aliquots of the metaphosphoric deproteinized reaction mixture under the conditions described, respectively, by P. P. Cohen in Biochem. J., 33, 551 (1939) and by D. D. Van Slyke and R. T. Dillon in Comp. rend. du Lab. Carlsberg, Ser. Chim., 22, 480 (1937).

In all experiments involving the use of radioactive isotopes or tracer elements it is a matter of primary concern whether the appearance of the tagged atom in a product represents a true metabolic reaction or has been introduced by a nonspecific exchange reaction. Oxalacetic acid, for example, breaks down to pyruvic acid and carbon dioxide in tissues, and it might be argued that any reaction involving this compound would yield a radioactive end product if the reaction were carried out in the presence of radioactive carbon dioxide. Such a possibility is difficult to control. However, the very large amounts of carbon dioxide assimilated argue against this explanation of the presence of radioactivity in the alpha-ketoglutaric acid. Also suggestive are experiments in which the possibility of an exchange between the carboxyl group of oxalacetic acid and carbon dioxide of the medium has been examined. In muscle, alpha-ketoglutaric acid is synthesized by the condensation of oxalacetate with pyruvate to form citrate and the subsequent oxidation of this compound to alpha-ketoglutarate; i.e., in terms of the citric acid cycle this is what occurs. Now, a formation of oxal- 
acetic acid from pyruvate and carbon dioxide in pigeon breast muscle does not occur, inasmuch as malonate will completely inhibit pyruvate utilization in the absence of added oxalacetic or other dicarboxylic acids. Under these circumstances the synthesis of muscle alpha-ketoglutarate in a radioactive bicarbonate medium would give rise to radioactive alpha-ketoglutarate only if a process of exchange between intermediates in the reaction (such as oxalacetate) and the carbonate of the medium took place. If the quantity of radioactivity found in muscle alpha-ketoglutarate is similar in magnitude to that found in the alpha-ketoglutarate synthesized by liver, it would eliminate the necessity for proposing a stoichiometric utilization of carbon dioxide in the process. When this reaction is studied in radioactive bicarbonate medium, the alpha-ketoglutarate formed is devoid of radioactivity. There is apparently no appreciable interchange between the carbon dioxide of the medium and the carboxyl group of oxalacetic acid or other intermediates. A similar negative result was obtained in studies of the equilibrium between pyruvic acid and carbon dioxide in the presence of carboxylase in which radioactive carbon dioxide and pressures as high as 300 atmospheres were used. Under these circumstances no interchange of carbon dioxide and the carboxyl of the pyruvate could be demonstrated. In view of these data, it seems most probable that the assimilation of carbon dioxide by minced liver is a true metabolic reaction, although the mechanism is still obscure.

The most promising line of attack of these problems lies probably in the attempt to isolate or simplify the enzyme systems involved. Recently attention has been directed again $(17,18)$ to the enzymic formation of acetylmethylcarbinol from pyruvate as a possible step in pyruvate oxidation. This reaction has two features of interest: first, it represents an anaerobic, i.e., non-oxidative, decarboxylation of pyruvate; secondly, it poses again the question of acetaldehyde as an intermediate. It is still too early to determine the relative importance of this reaction in pyruvate metabolism of muscle and other tissues. If it should prove to be of significance, the resulting clarity will undoubtedly be sufficient compensation for the revision that will have to be made in our current beliefs.

\section{REFERENCES}

1. Krebs, H. A., and Johnson, W. A., Enzymologia, 4, 148 (1937).

2. Krebs, H. A., and Eggleston, L. V., Biochem. J., 34, 442 (1940).

3. Krebs, H. A., Biochem. J., 34, 460 (1940).

4. Krebs, H. A., Biochem. J., 34, 775 (1940). 
5. Sмyтн, D. H., Biochem. J., 34, 1046 (1940).

6. Evans, E. A., Jr., Biochem. J., 34, 829 (1940).

7. Thunberg, T., Skand. Arch. Physiol., 24, 23 (1910).

8. Quastel, J. H., and Wooldridge, W. R., Biochem. J., 22, 689 (1928).

9. Evans, E. A., JR., Bull. Johns Hopkins Hosp., 69, 225 (1941).

10. Breusch, F. L., Biochem. J., 33, 1757 (1939).

11. Evans, E. A., Jr., and Slotin, L., J. Biol. Chem., 141, 439 (1941).

12. Wood, H. G., Werkman, C. H., Hemingway, A., and Nier, A. O., J. Biol. Chem. 139, 483 (1941).

13. Toeniessen, E., and Brinkmann, E., Z. physiol. Chem., 187, 137 (1930).

14. Wood, H. G., and Werkian, C. H., J. Bact., 30, 332 (1935); Biochem. J., 30,48 (1936); 32, 1262 (1938); 34, 129 (1940).

Wood, H. G., Werkitan, C. H., Hemingway, A., and Nier, A. O., J. Biol. Chem., 135, 789 (1940); 139, 365 (1941); 139, 377 (1941).

15. Evans, E. A., Jr., and Slotin, L., J. Biol. Chem., 136, 301 (1940).

16. Johnson, W. A., Biochem. J., 33, 1046 (1939).

17. Tankó, B., Munk, L., and Abonyı, I., Z. physiol. Chem., 264, 91 (1940).

18. Green, D. E., Westerfeld, W. W., Vennesland, B., and Knox, W. E., J. Biol. Chem., 140, 683 (1941). 


\title{
Transamination
}

\author{
PHILIP P. COHEN \\ University of Wisconsin
}

$T^{N}$ VIEW OF THE FACT that Braunstein (1) has recently reviewed in 1 some detail the results of experiments on transamination in his laboratory, no attempt will be made here to present tabular details of these studies. Unfortunately most of the publications issued since the review by Braunstein have not been available to the writer except in Chemical Abstracts, to which reference is made in the bibliography.

Many of the papers published in this field, being of a preliminary sort, are of uncertain value. Most investigators seem to be interested merely in demonstrating that transamination does or does not occur in a given tissue. Unfortunately the analytical methods usually employed permit little more than a qualitative demonstration of the reaction. Thus, in spite of a widespread interest in transamination, few reliable quantitative data are available that can be used for evaluating the role of this reaction in intermediary metabolism.

\section{Types of Transamination}

Transamination is a reaction between an alpha-amino and an alpha-keto acid resulting in the transfer of the amino group from the former to the latter. When the reaction is enzymatically catalyzed, the end products formed are an alpha-amino and an alphaketo acid, the former corresponding in structure to the original alpha-keto acid, and the latter to the original alpha-amino acid (Reaction 1).

$$
\begin{aligned}
& \mathrm{R}_{1} \cdot \mathrm{CH}\left(\mathrm{NH}_{2}\right) \cdot \mathrm{COOH}+\mathrm{R}_{2} \cdot \mathrm{CO} \cdot \mathrm{COOH} \rightleftarrows \mathrm{R}_{1} \cdot \mathrm{CO} \cdot \mathrm{COOH} \\
& +\mathrm{R}_{2} \cdot \mathrm{CH}\left(\mathrm{NH}_{2}\right) \cdot \mathrm{COOH}
\end{aligned}
$$

When carried out at boiling temperatures, the end products of transamination are usually an aldehyde corresponding to the original alpha-amino acid, an alpha-amino acid corresponding to the original alpha-keto acid, and carbon dioxide (Reaction 2).

$$
\begin{gathered}
\mathrm{R}_{1} \cdot \mathrm{CH}\left(\mathrm{NH}_{2}\right) \cdot \mathrm{COOH}+\mathrm{R}_{2} \cdot \mathrm{CO} \cdot \mathrm{COOH} \rightarrow \mathrm{R}_{1} \cdot \mathrm{CHO} \\
+\mathrm{R}_{2} \mathrm{CHNH} \mathrm{CHOH}_{2} \cdot \mathrm{COOH}+\mathrm{CO}_{2}
\end{gathered}
$$

Reaction 2 has been studied in some detail by Herbst, who has recently reviewed this subject (2). 
The present review will deal chiefly with the system represented by Reaction 1, and the use of the term "transamination" will be limited to this reversible, enzyme-catalyzed system.

\section{Discovery of Transamination Reaction}

Transamination as a biological reaction was first recognized by Needham (3), who found that glutamic and aspartic acids disappeared anaerobically when added to pigeon breast muscle without a decrease in amino nitrogen. She could find no increase in urea or ammonia nitrogen, but did observe an increase of succinic acid. She suggested that "possibly a combination of the amino group with some reactive carbohydrate residue takes place; then when splitting and oxidation occur the amino group is retained in the form of a new amino acid."

Unrecognized evidence for transamination was published from Szent-Györgyi's laboratory in 1936 and 1937. It was observed that the rate at which oxalacetic acid disappeared was greatly increased when glutamic acid was added to pigeon breast muscle (4) and to certain enzyme preparations from the same tissue (5).

Credit for the discovery of transamination goes to Braunstein and Kritzmann (6), who carried out the first detailed investigation of this reaction. They succeeded in showing that the reaction, termed "Umaminierung" by them, took place in pigeon breast muscle. The system represented in Reaction 3 was studied, and they were able to show its reversibility by isolation and chemical identification of the alanine and glutamic acid formed.

(3) $l(+)$-glutamic acid + pyruvic acid $\rightleftarrows \alpha$-ketoglutaric acid $+l(+)$-alanine.

\section{Substrates Active in Transamination}

Investigations by Braunstein and Kritzmann (7) led them to conclude that the enzymatic transfer of amino groups in pigeon breast muscle takes place between any alpha-amino acid (with the possible exception of glycine) and the dicarboxylic acids, alpha-ketoglutaric and oxalacetic, as well as between the dicarboxylic amino acids, glutamic and aspartic, and various alpha-keto acids. No amino group transfer was observed in the following systems: (1) between monocarboxylic alpha-amino acids and monocarboxylic alpha-keto acids; (2) from amines and peptides; or (3) from alpha-amino dicarboxylic acids to ketones, hydroxy-ketones, or aldehydes. The analytical method employed in this study for measuring the formation or dis- 
appearance of glutamic acid was an adaptation of the Foreman (8) method, which has recently been shown by Zorn (9) to be unreliable for studying transamination.

In contrast to certain of the above findings Cohen (10) found that of twenty-one different alpha-amino acids studied in pigeon breast muscle $l(-)$-aspartic acid and $l(+)$-alanine were the most active in forming glutamic acid in the presence of alpha-ketoglutaric acid. Alpha-aminobutyric acid and $l(+)$-valine were slightly active, but none of the remaining amino acids was appreciably active. Of a series of alpha-keto acids studied, oxalacetic and pyruvic acids were the most active in causing the anaerobic disappearance of glutamic acid. Alpha-ketobutyric and mesoxalic acids were slightly active, but no activity was observed with alpha-ketovaleric, alpha-ketocaproic, acetoacetic, and laevulic acids. Moreover, no transamination was observed between alpha-ketoglutaric acid and a variety of amino compounds other than alpha-amino acids. The formation and disappearance of glutamic acid were determined by the method of Cohen (11). The specificity and accuracy of this method has recently been confirmed $(12,13)$.

$d$-Amino Acids.-Amino acids of the $d$ series are not active in transamination $(10,14,15,16)$. Activity with $d$-amino acids has been reported by Braunstein (1), Braunstein and Azarkh (17), and Euler et al. (18).

Peptides.-The role of peptides as transamination substrates is of considerable interest. As noted above, Braunstein and Kritzmann (7) observed no activity with various peptides. In the case of transaminase preparations Cohen (14) was unable to demonstrate any appreciable transamination between glutathione and oxalacetic acid. On the other hand Ågren (19) reported that in minced cattle diaphragm muscle transamination takes place between alphaketoglutaric acid and the peptides glycylaminobenzoic acid and valylglycine. Valylglycine was found to be as active as alanine (about 30 per cent transamination), while glycylaminobenzoic acid was less active. Agren employed the same method as Braunstein and Kritzmann for measuring transamination. In view of the latter workers' observation that glycine interfered with this determination (1) by being carried down in the dicarboxylic acid amino nitrogen fraction, it is possible that the results obtained by Agren with glycine dipeptides are due to this fact. No control experiments of glycine dipeptides plus tissue are reported.

"Primary" and "Secondary" Substrates.-According to Braunstein 
the substrates available for transamination can be considered as consisting of two types:

1. "Primary" or "active" substrates. These have a high affinity for the enzyme and react with mono- or dibasic acids after adsorption. Primary substrates are the dibasic alpha-amino or alpha-keto acids, and include compounds such as cysteic acid.

2. "Secondary" or "passive" substrates. This group includes all the alpha-amino and alpha-keto monobasic acids which have no direct affinity for the enzyme and serve only as "reaction partners" for the primary substrates.

This idea of Braunstein can be extended to mean that only the dibasic alpha-amino or -keto acids are activated by the enzyme. This would suggest that the dibasic alpha-amino and -keto acids act as prosthetic groups, which in the presence of the enzyme react with the secondary substrates. That is, the secondary substrates have an affinity for the enzyme only when the "primary" substrates have become activated. This concept is not unreasonable, but it appears unnecessary to classify the substrates as active or passive. From Braunstein's point of view this is essential, since he has to account for the activity of the large number of monobasic alpha-amino and -keto acids which he reports to be active in transamination. From the writer's point of view this concept is unnecessary, since his experimental data lead to the conclusion that transamination is essentially a limited reaction and concerned chiefly with the dibasic alphaamino and -keto acids. The activity of other compounds can be explained on the basis of different affinities for the enzyme. Thus with large amounts of tissue and long incubation periods other amino acids show a small amount of activity (20).

"Catalytic" Transamination.-Braunstein (1) and Braunstein and Kritzmann (21) have reported that the addition of small amounts of a dibasic alpha-amino or alpha-keto acid (as little as M/16000) causes transamination to take place in pigeon breast muscle brei between lysine and pyruvic acid. The latter system alone is inactive. With glutamic acid, the reaction is pictured as proceeding in the following manner:

(3) glutamic acid + pyruvic acid $\rightarrow \alpha$-ketoglutaric acid + alanine

(4) $\alpha$-ketoglutaric acid + lysine $\rightarrow$ glutamic acid +2 -keto-6-aminocaproic acid The net effect of this would be:

(5) lysine + pyruvic acid $\rightarrow$ 2-keto-6-aminocaproic acid + alanine

The effectiveness of so low a concentration as $\mathrm{M} / 16000$ is remark- 
able in view of the fact that, as Braunstein himself points out, pigeon breast muscle brei has a much higher content of dibasic alphaamino and -keto acids normally present (11). Yet Braunstein reports that reaction 5 will not take place without the addition of small amounts of catalyst. The writer $(10,14)$ was unable to demonstrate a similar catalytic effect with either pigeon breast muscle or purified transaminase by using the system:

(6) $l(-)$-aspartic acid + pyruvic acid $\rightleftarrows$ oxalacetic acid $+l(+)$-alanine.

Braunstein (1) and Bychkov (22) have reported that cysteic acid and phosphoserine are active in transamination in pigeon breast muscle. Using purified transaminase, the writer confirmed the activity of cysteic acid, but phosphoserine was found to be inactive. Of interest, however, is the report by Braunstein (1) that neither of these two compounds is active with purified enzymes (glutamic and aspartic aminopherases).

\section{Preparation and Properties of Transaminating Enzymes}

Kritzmann $(23,24,25)$ has described in some detail the preparation and properties of purified transaminating enzymes from pigeon breast and pig heart muscles. According to her, two distinct systems exist, one of which is concerned with glutamic acid (and alphaketoglutaric acid) and the other with aspartic acid (and oxalacetic acid). The former enzyme is called glutamic aminopherase and the latter aspartic aminopherase. Both enzymes are reported to require co-factors, present in muscle kochsaft, whose chemical constitutions are still unknown but which are similar if not identical for the two systems. Aspartic aminopherase is thought to be a more labile system, since it is claimed that muscle suspensions lose their transaminating activity on dilution more rapidly with aspartic acid than with glutamic acid.

The following are some of the properties of the aminopherases found by Kritzmann (25): 1. Purification by adsorption, salting out, or dialysis results in inactivation. 2. Reactivation follows on addition of boiled muscle extracts or ultrafiltrates. 3. To be effective, the glutamic-aspartic aminopherases must contain a thermostable, low molecular weight activator or coenzyme. 4. Denaturation by acetone, ethyl alcohol, or methyl alcohol leads to an irreversible inactivation. 5 . Heating at $80^{\circ} \mathrm{C}$. for five minutes causes a 50 per cent decrease in activity. 6. $\mathrm{pH}$ activity range is 5.5-8.5, with an optimum 
at 7.4. 7. Glutamic aminopherase is best obtained from pig heart muscle, aspartic aminopherase from coarsely ground pea seedlings. If the latter are finely ground, both aspartic and glutamic aminopherases are obtained.

Transaminating enzyme preparations from pig heart and pigeon breast muscle have recently been studied by Cohen $(14,26)$. It was found that the activity of these preparations was greatest with the systems, $l(+)$-glutamic acid plus oxalacetic acid, and alpha-ketoglutaric acid plus $l(-)$-aspartic acid. That is, the enzyme was most active in catalyzing a reaction in which both glutamic and aspartic acids (and the corresponding alpha-keto acids) were substrates. The addition of pigeon breast muscle kochsaft was without influence on the rates of reactions 3,6 or 7 .

(7) $l(+)$-glutamic acid + oxalacetic acid $\rightleftarrows \alpha$-ketoglutaric acid $+l(-)$-aspartic acid

Reaction 7 was catalyzed at a rapid rate, the $Q_{T}$ values being of the order of 1600. "The position of equilibrium for this system was far to the right, with an equilibrium constant of about 3 . Reaction 3 was catalyzed at a much slower rate by transaminase, the $\mathrm{Q}_{\mathrm{T}}$ values being of the order of 300 . The equilibrium constant was about 1 . Reaction 6 was not catalyzed by transaminase.

The following are some properties of transaminase: 1 . The enzyme is best prepared from pig heart muscle and pigeon breast muscle. 2. Transaminase can be dried by rapid lyophilization at low temperatures. Such preparations remain active for as long as six weeks at room temperature (63). 3. Purification by salting out or dialysis results in inactivation. However, solutions of the enzyme can be further purified by adsorption on calcium phosphate (63). 4. Transaminase has an optimum activity at $40^{\circ} \mathrm{C}$. and at $\mathrm{pH} 7.5$. The Michaelis constant with the substrates glutamic and oxalacetic acids is 0.0138 M. 5. Muscle kochsaft, diphosphopyridine nucleotide, thiamin, and cocarboxylase are without influence.

v. Euler et al. (27) have stated that the transaminating enzyme does not require coenzymes or apparently any other cofactor dissociable at a neutral reaction.

Substrate Specificity.-Experiments with purified enzyme preparations (transaminase) showed essentially the same substrate specificity as with pigeon breast muscle (14). Thus with alpha-ketoglutaric

${ }^{\circ} \mathrm{Q}_{\mathrm{T}}=\mathrm{Q}_{\text {transamination }}=$ microliters substrate transaminated per mg. dry weight per hour. 
acid only $l(-)$-aspartic acid, $l(+)$-alanine, and $l(-)$-cysteic acid were active, while with oxalacetic acid only $l(+)$-glutamic and $l(-)$-cysteic acids reacted. A few experiments have been reported by Braunstein ( 1 ) in which glutamic acid formation from alphaketoglutaric acid and different amino acids was measured with both pigeon breast muscle and purified enzyme preparations. Of interest are the findings that while $l(+)$-alanine shows a slightly smaller activity with the pigeon breast muscle, $l(+)$-valine, $l(-)$-leucine, and $l(+)$-isoleucine react to only about one-third the extent with purified enzyme. These differences, no doubt, reflect the inadequacy of the analytical method employed in these investigations.

From the studies with purified transaminase $(12,14)$ it appeared that the chief substrates for this enzyme are those represented in reaction 7 . Aside from pyruvic acid and $l(+)$-alanine, no monobasic alpha-amino or alpha-keto acids were found to be active. However, dibasic alpha-keto and alpha-amino acids other than those shown in reaction 7 are active. Thus $l(-)$-cysteic acid will react with both oxalacetic and alpha-ketoglutaric acids. Glutathione does not react with oxalacetic acid.

\section{Mechanism of Transamination}

The mechanism by which the intermolecular transfer of the amino group takes place is not known. Following Herbst's (2) idea of the mechanism of non-enzymatic transamination, Braunstein and Kritzmann (6) have postulated the formation of an intermediate Schiff's base, and picture the reaction as follows:

SCHEME I
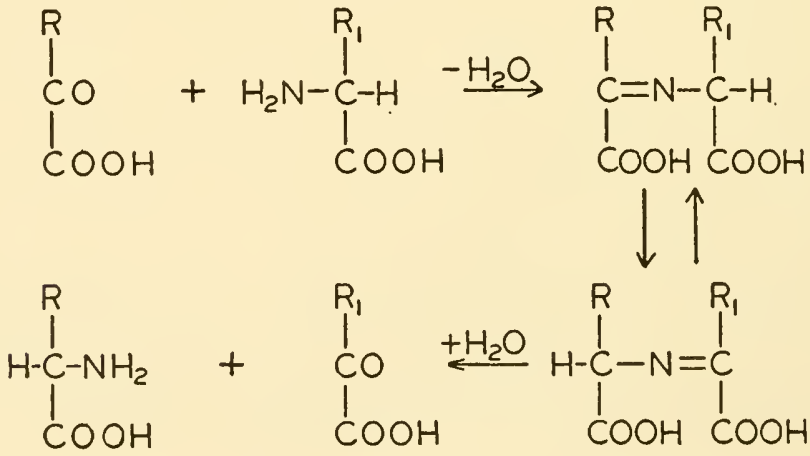
The inability of purified transaminase preparations to dehydrogenate glutamic or aspartic acids in the presence of suitable hydrogen acceptors, and the absence of free ammonia during the course of the reaction suggest that the amino group is transferred through an intermediate complex of the Schiff's base type.

Karrer et al. (28) have investigated the metabolism of octopin, following the suggestion of Knoop and Martius (29) that octopin represents a naturally occurring intermediate compound of transamination. Karrer et al. found that fresh liver brei (which they state contains an $l$-amino acid dehydrogenase) was capable of dehydrogenating octopin. $d$-Amino acid dehydrogenase preparations were not active, indicating that the alanine was of the $l$-form. Since the biological synthesis of octopin involves an intermediate reduction of a Schiff's base to an $\alpha, \alpha^{\prime}$-imino dicarboxylic acid, the authors conclude that transamination may involve intermediate reduction of the Schiff's base with subsequent dehydrogenation. The reaction is pictured as follows:

SCHEME II<smiles>[R]C(=[C+]CO)C(=O)O</smiles><smiles>[R]C(=NC([R])C(=O)O)C(=O)O</smiles><smiles>[R]C(NC([R])C(=O)O)C(=O)O</smiles><smiles>[R]C(=O)C(=O)O</smiles><smiles>[R]C(=NC([R])C(=O)O)C(=O)O</smiles>

It has the advantage of providing a mechanism for the explanation of the double bond shift between the nitrogen and the two alpha carbon atoms.

As previously pointed out, the evidence for the existence of a 
cofactor for transaminase is still inconclusive. Should an oxidizable and reducible cofactor prove to be involved, its function might be that of acting as the hydrogen acceptor and donator in the scheme of Karrer et al. (Scheme II). However, as has been said, none of the known cofactors has any influence on transaminase activity, notwithstanding the fact that the method of its preparation is such as to remove practically all the known cofactors with the possible exception of flavinadenine dinucleotide. Of interest in this connection is the writer's unpublished observation that the activity of transaminase preparations, fractionated by various methods for purposes of purification, is associated with those fractions showing a green fluorescence, similar to that obtained with flavoproteins.

\section{Transamination in Different Tissues}

As has been said, much of the available data on transamination is of a qualitative nature. Not only must it be demonstrated that transamination occurs in a given tissue, but the rate of the reaction in terms of unit weight of that tissue must be known. Thus a significant amount of transamination may be shown to take place in certain cases with large amounts of tissue and long incubation periods, but calculation of the rates in terms of $Q_{T}$ would reveal a value so low as to cast doubt on the significance of this reaction in the metabolism of that tissue. It is thus essential to have accurate data on the rate of transamination in different tissues before assigning to it a role in intermediary metabolism. Unfortunately very few such data are available.

Animal Tissues.-Transamination in different animal tissues was first studied by Kritzmann (30). Using the system glutamic acid plus pyruvic acid, she reported transaminase activity in liver, kidney, skeletal muscle, heart muscle, and brain, but none in smooth muscle (chicken gizzard), lung, erythrocytes, and yeast. There was questionable activity in the case of malignant tissue. Values of $\mathrm{Q}_{\mathrm{T}}$ calculated from these data are of the order of 1.5-2.0 for the more active tissues. Similar studies were carried out on a variety of tissues by Cohen (31).

A quantitative study of the rate of transamination in different rat tissues was recently carried out by Cohen and Hekhuis (15). As can be seen from Table 1, the rates, expressed in terms of $Q_{T}$, are very high in most tissues with the substrates glutamic acid plus oxalacetic acid. The $Q_{T}$ values are higher than the succinoxidase 
$Q$ values for the same tissues (32) and in most instances exceed the cytochrome oxidase $Q$ values $(32,33)$.

The $Q_{\mathrm{T}}$ values with the systems glutamic acid plus pyruvic acid, and aspartic acid plus pyruvic acid, are comparatively low in all tissues studied except liver. The higher rates of transamination in liver are probably due to the fact that pyruvic acid is converted into oxalacetic and alpha-ketoglutaric acids in this tissue $(15,34$, $35,36)$.

Table 1.-Values of $\mathrm{Q}_{\mathrm{T}}$ in different rat tissues

\begin{tabular}{lcccc}
\hline \hline Tissue & & $\begin{array}{c}\text { Glutamic acid } \\
\text { Oxalacetic acid }\end{array}$ & $\begin{array}{c}\text { Glutamic acid } \\
\text { Pyruvic acid }\end{array}$ & $\begin{array}{c}\text { Aspartic acid } \\
\text { Pyruvic acid }\end{array}$ \\
\hline Heart muscle . . . . . . & 425 & 7 & 7 \\
Skeletal muscle . . . . . & 316 & 13 & 1 \\
Brain. . . . . . . . . . . & 260 & 2 & 8 \\
Liver . . . . . . . . . . & 245 & 46 & 10 \\
Kidney . . . . . . . . . & 245 & 3 & 3 \\
\hline
\end{tabular}

It is apparent from the data in Table 1 that transamination is chiefly concerned with the substrates glutamic acid plus oxalacetic acid. The metabolic importance of this will be discussed later.

Breusch (37) reported some experiments on transamination in different tissues with the system glutamic acid plus oxalacetic acid. The following $\mathrm{Q}_{\mathrm{T}}$ values for various minced cat tissues were calculated from his data: muscle, 19.7; liver, 14.1; kidney, 22.5; lung, 22.5; embryonic muscle, 14.1; brain, 19.7; and washed erythrocytes, 2.8. These values are all much lower than those reported for cat tissues by Cohen and Hekhuis (16). Breusch claims that lung tissue is an excellent source of transaminase, since it is free of many dehydrogenase systems. Cohen and Hekhuis, on the contrary (15), found lung to have a low transaminase activity. The method of oxalacetic acid determination used by Breusch (4) for measuring transamination is far from satisfactory and probably accounts for the results obtained.

Karayagina (38) found that $l(-)$-aspartic acid reacts with pyruvic acid to form alanine in skeletal and cardiac muscles, in liver, kidney, and brain, but not in testes.

Malignant and Embryonic Tissues.-Euler, Günther, and Forsman (39) and Braunstein and Azarkh (17) reported low transamination values for tumors. The former workers used a qualitative ana- 
lytical method for measuring the disappearance of oxalacetic acid. The latter workers studied chiefly the reactions, glutamic acid plus pyruvic acid, and alpha-ketoglutaric acid plus different amino acids.

Quantitative studies of the rate of transamination in different tumors and embryonic tissue have been carried out by Cohen and Hekhuis (16). In their study a series of mouse tumors and cat embryonic tissues were shown to have low rates of transamination as compared with normal adult tissue. Further, the writer has shown (63) that the transaminase activity of liver from rats fed dimethylaminoazobenzene decreases progressively to a low value in the liver tumors arising in these animals. It thus appears that rapid growth, or increased protein synthesis, is associated with a low transaminase activity.

Plant Tissues.-Euler et al. (27) reported transaminase activity in higher plants, but no data were published in support of this statement. Virtanen and Laine (41) state that transamination between oxalacetic acid and alanine takes place in crushed pea plants. Transaminase activity in extracts prepared from pea, lupine, and pumpkin seedlings has been reported by Kritzmann (24). Cedrangolo and Carandante (42) studied transamination in leguminous and graminaceous plants. Dialyzed extracts of seeds and sprouts were used and were prepared by the same method as that employed by Adler and Sreenivasaya (43) for the preparation of formicodehydrogenase. The systems $l(-)$-aspartic acid plus pyruvic acid, $l(-)$-aspartic acid plus alpha-ketoglutaric acid, and alpha-ketoglutaric acid plus $l(+)$-alanine were investigated. Experimental data are not presented in this paper, but rather the relative velocities of the above reactions in graminacae seeds and sprouts as compared with leguminous seeds and sprouts, which are assigned an arbitrary value of 100 . The graminacae extracts are reported to be 1.5 to 2.5 times as active as those from legumes. According to these authors, the lack of aspartic acid utilization by graminacae, reported by Virtanen, cannot be due to the lack of a transaminating enzyme. Of interest is the finding of these workers that dialyzed plant extracts are active. As previously mentioned, Kritzmann (25) reported that dialysis inactivates transaminating enzymes from plant sources.

Experiments with Chlorella (63) by the author failed to show any evidence of transaminase activity with the systems alphaketoglutaric acid plus $l(+)$-alanine, and alpha-ketoglutaric acid plus $l(-)$-aspartic acid. 
Wyss (44) observed formation of considerable amounts of aspartic acid when oxalacetic acid was added to crushed pea nodules. The amount of aspartic acid found was somewhat greater when alanine was also added. Whether the aspartic acid formation is due to transamination or to some other reaction is not certain.

Yeast and Bacteria.-Adler, Günther, and Everett (45) reported transaminase activity in yeast extracts. Adler et al. (46) state that Bacterium coli (Escherichia coli) suspensions form oxalacetic acid from alpha-ketoglutaric acid and aspartic acid, and that lactic acid bacilli are capable of transamination, though at a slower rate than Esch. coli. No experimental data are given in either of the above papers.

Experiments by the writer (63) showed that Lebedev juice prepared from brewer's yeast was active in catalyzing the reaction alpha-ketoglutaric acid plus $l(-)$-aspartic acid, but not the reaction, alpha-ketoglutaric acid plus $l(+)$-alanine. Baker's yeast showed no activity with either system. Experiments with Esch. coli suspensions (63) demonstrated that no transamination occurred between $l(+)$ glutamic acid and pyruvic acid, but did take place between $l(+)$-glutamic acid and oxalacetic acid. $Q_{T}$ for the latter reaction was 17.2 .

Transamination in vivo.-Kritzmann (40) found that intravenous, intramuscular, or intraperitoneal injection of glutamic acid into rabbits, pigeons, and white mice causes the rapid appearance of alanine in the blood and the tissues. The pyruvic acid necessary for this reaction is endogenous. The injection of alpha-ketoglutaric acid plus alanine resulted in the formation of glutamic acid. No transamination occurs in the blood itself. The failure of blood cells to catalyze transamination has also been observed by the writer (63).

\section{Influence of Various Substances on Transamination}

Inhibitors.-None of the well-known inhibitors has any marked effect on transamination with the exception of cyanide, which in high concentrations $(0.05 \mathrm{M})$ causes up to 80 per cent inhibition. This is in all probability due to the formation of cyanohydrin. However, at $0.001 \mathrm{M}$ concentration cyanide still causes an inhibition of about 30 per cent, indicating that an effect on the enzyme system may occur. Malonate, pyrophosphate, sodium fluoride, iodoacetate, bromoacetate, arsenious oxide, and octyl alcohol have little or no influence on transamination in pigeon breast muscle (10).

With transaminase no inhibition of transamination between glu- 
tamic acid and oxalacetic acid was noted in the presence of malonate, succinate, pyrophosphate, and citrate (14).

In contrast to the above findings with di- and tribasic acids Braunstein (1) reported that small concentrations of dibasic acids competitively inhibit "catalytic" transamination. The latter refers to the catalytic effect of small concentrations of a dibasic alphaamino or alpha-keto acid on transamination between a monobasic alpha-amino acid and a monobasic alpha-keto acid. The author's attempts to corroborate this finding of Braunstein's have not been successful $(10,14)$.

Vysshepan (47) found that the activity of glutamic aminopherase was inhibited by the following reagents (molar concentrations): quinone (0.01); potassium cyanide (0.01); glutathione (0.002-0.004); cations of calcium, barium, and strontium (0.02); mercury and silver (0.0001). Reagents which are relatively harmless are narcotics, sodium fluoride, monoiodo- and monobromo-acetic acids, arsenite, arsenate and selenite; the anions, chloride, bromide, iodide, acetate, nitrate, carbonate, sulfate (0.01); ascorbic acid (0.01), hydrogen sulfide, cysteine, ferrous ion, semicarbazide, phenylhydrazine and hydroxylamine. Sober and Cohen (64) observed no inhibition of transaminase by glutathione $(0.005 \mathrm{M}$.).

It is apparent that thus far no specific inhibitor is available for transaminase. The discovery of such a compound would aid greatly in elucidating the physiological role of transamination.

Hormones.-Transamination with purified transaminase and the system glutamic acid plus oxalacetic acid is uninfluenced by high concentration of the following: insulin (crystalline and zinc compounds), desoxycorticosterone, cortical extract, anterior pituitary extract, estradiol, androsterone, testosterone, and stilbestrol (63).

Carcinogens.-No effect on transaminase activity was observed with methylcholanthrene and dimethylaminoazobenzene (63).

Vitamins.-Thiamin and cocarboxylase are without influence on transaminase activity (63). However, a decrease in activity was observed in minced breast muscle from $B_{1}$ deficient pigeons with the system glutamic acid plus pyruvic acid (63). Similar findings have recently been published by Kritzmann (48). Barron (49), on the other hand, found no decrease in transamination in liver from $B_{1}$ deficient rats. These results are not necessarily contradictory, since Barron and his coworkers employed somewhat different experimental conditions (personal communication). Investigations are at 
present under way by the writer to determine the influence of other vitamin deficiencies on this reaction.

\section{Role of Transamination in Intermediary Metabolism}

The exact role which transamination plays in intermediary metabolism is still not clear. The substrates of this reaction are highly reactive and participate in many different rapid metabolic processes. Obviously if transamination is to play a significant role in cellular metabolism it must be shown to proceed at a rate rapid enough to be quantitatively significant. From Table 1 it is apparent that truly rapid rates are seen only with the system glutamic acid plus oxalacetic acid. The rapid rates at which oxalacetic, alphaketoglutaric, and pyruvic acids participate in non-transaminating reactions make it highly doubtful whether reactions 3 and 6 ever proceed fast enough to participate in the metabolism of these compounds. This may not apply to liver and pigeon breast muscle.

Among the possible metabolic reactions which transamination may influence are those of protein and amino acid synthesis and degradation, glycolysis, and hydrogen transport.

\section{PROTEIN AND AMINO ACID SYNTHESIS AND DEGRADATION}

Animal Tissues.-An attractive theory of amino acid synthesis and degradation in plant and animal tissues has been proposed by Braunstein (1). According to this theory amino acids are synthesized or degraded by the transamination reaction in conjunction with the glutamic dehydrogenase system of Euler et al. (27) and Dewan (50). The latter system serves the two functions of synthesizing glutamic acid for transamination with alpha-keto acids to yield new amino acids, and of oxidizing the glutamic acid formed from alphaketoglutaric acid and different amino acids. Both enzyme systems are present in most tissues, although in varying amounts. Thus transaminase is higher in muscle than in liver, whereas glutamic dehydrogenase is higher in liver than in muscle.

Braunstein and Bychkov (51) have reported production of ammonia from $l(+)$-alanine when the latter is incubated with alphaketoglutaric acid, glutamic aminopherase, glutamic dehydrogenase, pyocyanine, and cozymase. About 12.5 per cent of the theoretical yield was realized after three hours' incubation. These workers pointed out that the above system is a cell-free model of $l$-amino acid dehydrogenase, which to date has not been demonstrated to 
represent a single enzyme system, as is the case with $d$-amino acid dehydrogenase.

The mechanism of deamination of $l$-amino acids in liver and kidney can be explained on the basis of the above theory. However, the failure of the other tissues to oxidize amino acids other than glutamic acid to any appreciable extent is difficult to explain, since, as was said above, the two necessary enzyme systems are present in most tissues. Inasmuch as the theory rests in large measure on Braunstein's claim that all amino acids are active in transamination, unequivocal proof for this would seem desirable. As previously pointed out, experiments by the writer have shown transamination to be a limited reaction rather than a general one. Further, the author has found that homogenized liver and kidney, fortified with cozymase and methylene blue, failed to show any appreciable yields of ammonia from various combinations of alpha-ketoglutaric acid and $l$-amino acids (63).

A possible metabolic relationship between transamination and protein synthesis in animal tissues has been pointed out by Linderstrom-Lang (52). On the basis of the plausible assumption that the synthesis of protein occurs by a metabolic mechanism other than the reversal of proteolysis, he postulates the following scheme:

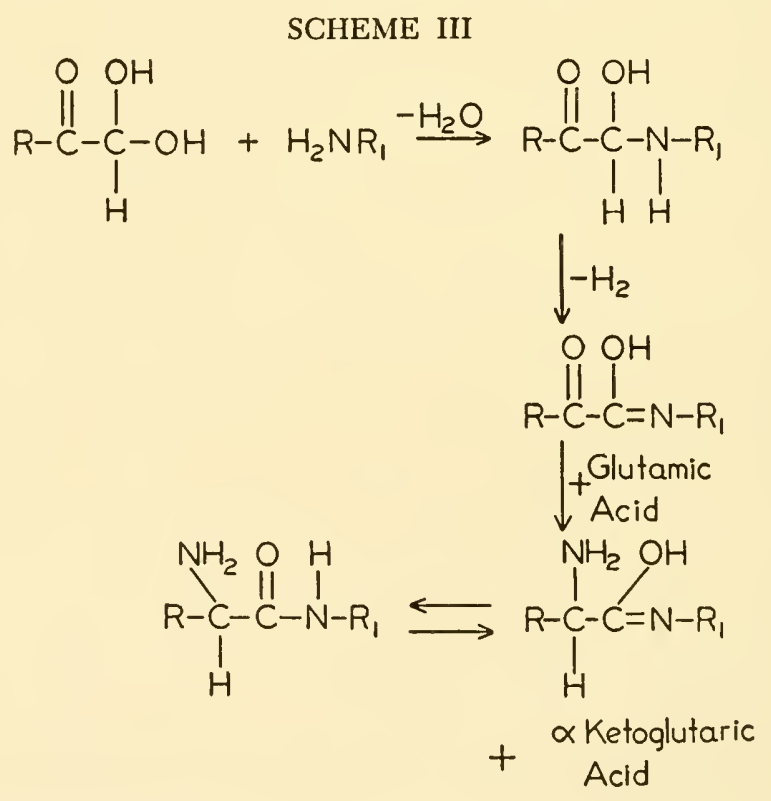


As can be seen from this scheme, a keto-aldehyde reacts with an amino acid to form a ketonic Schiff's base. The latter then reacts with glutamic acid via the transamination reaction to yield a peptide. Ågren (19) has recently published unconvincing evidence in support of this.

On the basis of the above reaction it would be expected that in tissues where rapid protein synthesis was taking place, e.g., embryonic and tumor tissue, the transaminase activity would be higher than in normal adult tissues. Actually the reverse has been found to be the case (16). Thus it was observed that in tumors and embryonic tissue the transaminase activity was low as compared with normal adult tissues. This apparent inverse relationship between protein synthesis and transamination suggests that the latter reaction may serve as a controlling mechanism in protein synthesis.

Plant Tissues.-The possible role of transamination in plant protein synthesis is suggested from the following scheme of Virtanen and Laine (41) for leguminous plants:

SCHEME IV

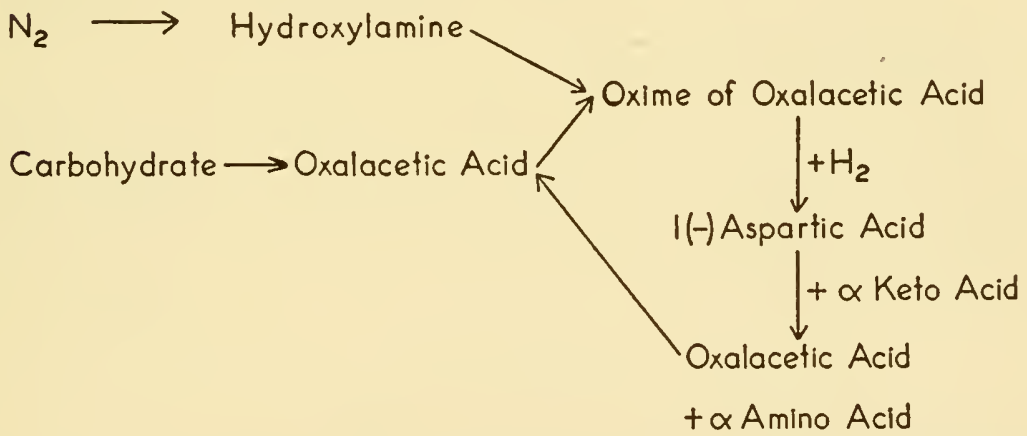

Experimental evidence for the above scheme has been reported by Virtanen and Laine $(41,53)$ and has been critically examined by Wilson (44). However, careful quantitative studies on transamination in plant tissues have not as yet been carried out. Until this is done the role of transamination in plant tissues will continue to remain obscure.

\section{OTHER REACTIONS}

Transamination and Glycolysis.-Krebs (54) and Weil-Malherbe (55) observed that glycolysis in retina and brain tissue was in- 
hibited by glutamic acid. This effect has been studied in more detail recently by Grodzensky (56), who was able to show that anaerobic glycolysis in pigeon breast muscle was also inhibited (20-50 per cent) by glutamic acid. Further, he was able to demonstrate that the inhibition was due to the conversion of pyruvic acid to alanine by transamination of the former with glutamic acid.

The influence of glutamic acid on glycolysis in tumors has been investigated by the author (63). Inhibitions of the order of 10-15 per cent were observed. These results are in keeping with the finding that tumors have a low transaminase content (16).

Transamination and Hydrogen Transport.-Transamination is not only a very rapid reaction but it is also concerned chiefly with those substances that are known to play key roles in intermediary metabolism. Thus oxalacetic, alpha-ketoglutaric, glutamic, and aspartic acids all catalytically influence respiration $(3,4,57,58,59)$. Further, glutamic acid has been found to function as a hydrogen carrier not only because its dehydrogenase can act with both di- and triphosphopyridine nucleotides $(27,60)$ and so can couple with other diand triphosphopyridine nucleotide-catalyzed systems $(60,61)$, but also because of its role in a dismutation reaction involving alphaketoglutaric acid and ammonia (62).

It would thus appear that the chief role of transamination may be that of rapidly interconverting certain of the respiratory mediators. That the transamination reaction is fast enough to compete successfully with other metabolic reactions involving the same substrates has been previously indicated.

\section{REFERENCES}

1. Braunstein, A. E., Enzymologia, 7, 25 (1939).

2. Herbst, R. M., Symposia on Quantitative Biology, 6, 32 (1938).

3. Needhalr, D. M., Biochem. J., 24, 208 (1930).

4. Annau, E., Banga, I., Blazso, A., Bruckner, V., Laki, K., Straub, F. B., and Szent-Györgyi, A., Z. physiol. Chem., 244, 105 (1936).

5. BANGA, I., and Szent-Györgyi, A., Z. physiol. Chem., 245, 118 (1937).

6. Braunstein, A. E., and Kritzmann, M. G., Enzymologia, 2, 129 (1937).

7. Braunstein, A. E., and Kritzmann, M. G., Biochimia, U.S.S.R., 3, 603 (1938).

8. Foreman, F. W., Biochem. J., 8, 463 (1914).

9. Zorn, K., Z. physiol. Chem., 266, 239 (1940).

10. Cohen, P. P., Biochem. J., 33, 1478 ( 1939 ).

11. Cohen, P. P., Biochem. J., 33, 551 (1940).

12. Macara, T. J. R., and Plimmer, R. H. A., Biochem. J., 34, 1431 ( 1940 ).

13. Woodward, G. E., Reinhart, F. E., and Dohan, J. S., J. Biol. Chem., 138, 677 (1941).

14. Colien, P. P., J. Biol. Chem., 136, 565 (1940). 
15. Cohen, P. P., and Hekruis, G. L., J. Biol. Chem., 140, 711 (1941).

16. Cohen, P. P., and Hekruis, G. L., Cancer Research, 1, 620 (1941).

17. Braunstein, A. E., and Azarkh, R. M., Nature, 144, 669 (1939).

18. v. Euler, H., Hellström, H., Günther, G., Elliott, L., and Elliott, S., Z. physiol. Chem., 259, 201 (1939).

19. ÄGren, G., Acta Physiol. Scand., 1, 233 (1940).

20. Cohen, P. P., Proc. Am. Soc. Biol. Chem., J. Biol. Chem., 133, xx (1940).

21. Braunstein, A. E., and Kritzmann, M. G., Biochimia, U.S.S.R., 4, 168 (1939). Chem. Abstr. 34, 1694 (1940).

22. Bychkov, S. M., Biochimia, U.S.S.R., 4, 189 (1939). Chem. Abstr., 34, 1694 (1940).

23. Kritzmann, M. G., Biochimia, U.S.S.R., 3, 603 (1938).

24. KritzmanN, M. G., Nature, 143, 603 (1939).

25. Kritzmann, M. G., Biochimia, U.S.S.R., 4, 667 (1939). Chem. Abstr., 34, $5865(1940)$.

26. Cohen, P. P., J. Biol. Chem., 136, 585 (1940).

27. v. Euler, H., Adler, E., Günther, G., and Das, N. B., Z. physiol. Chem., 254, 61 (1938).

28. Karrer, P., Koenig, H., and Legler, R., Helv. Chim. Acta., 24, 127 (1940).

29. Knoop, F., and Martius, C., Z. physiol. Chem., 254, I ( 1938 ).

30. Krutzmann, M. G., Enzymologia, 5, 44 ( 1938 ).

31. Cohen, P. P., Am. J. Physiol., 126, 467 (1939).

32. Elliott, K. A. C., and Grieg, M. E., Biochem. J., 32, 1407 (1938).

33. Schulze, M. O., J. Biol. Chem., 129, 727 (1939).

34. Evans, E. A., Jr., and Slotin, L., J. Biol. Chem., 136, 301 (1940).

35. Krebs, H. A., and Eggleston, L. V., Biochem. J., 34, 1383 (1940).

36. Wood, H. G., Werkman, C. H., Hemingway, A., and Nier, A. O., J. Biol. Chem., 139, 483 (1941).

37. Breusch, F. L., Biochem. J., 33, 1757 ( 1939 ).

38. Karayagina, M. K., Biochimia, U.S.S.R., 4, 168 (1939). Chem. Abstr., 34, 1694 (1940).

39. v. Euler, IH., Günther, G., and Forsinan, N., Z. f. Krebsforsch., 49, 46 (1939).

40. Kritzmann, M. G., Biochimia, U.S.S.R., 4, 184 (1939). Chem. Abstr., 34, 1694 (1940).

41. Virtanen, A. I., and Laine, T., Nature, 141, 748 (1938).

42. Cedrangolo, F., and Carandante, G., Boll. soc. ital, biol. sper., 15, 482 (1940).

43. Adler, E., and Sreenivasaya, M., Z. physiol. Chem., 249, 24 ( 1937 ).

44. Wyss, O., quoted by Perry W. Wilson in The Biochemistry of Symbiotic Nitrogen Fixation (University of Wisconsin Press, Madison, Wisconsin, $1940)$, p. 175.

45. Adler, E., Günther, G., and Everett, J. E., Z. physiol. Chem., 254, 27 (1938).

46. Adler, E., Hellström, V., Günther, G., and v. Euler, H., Z. physiol. Chem., 255, 14 (1938).

47. Vyssiepan, E. D., Biochimia, U.S.S.R., 5, 271 (1940). Chem. Abstr., 35, 4788 ( 1941$)$.

48. Kuitzmann, M. G., Biochimia, U.S.S.R., 5, 281 (1940). Chem. Abstr., $35,4788$ ( 1941$)$.

49. Barnon, E. S. G., Ann. Rev. Biochem., 10, 15 (1941).

50. Dewan, J. G., Biochem. J., 32, 1378 (1938).

51. Braunstein, A. E., and Bychkov, S. M., Nature, 144, 751 (1939). 
52. Lindenstr $\varnothing$ m-Lang, K., Ann. Rev. Biochem., 7, 37 (1939).

53. Virtanen, A. I., and Laine, T., Biochem. J., 33, 412 (1939).

54. Krebs, H. A., Biochem. J., 29, 1951 (1935).

55. Weil-Malherbe, H., Biochem. J., 32, 2257 (1938).

56. Grodzensky, D. E., Bull. biol. med. exptl. U.S.S.R., 9, 116 (1940). Chem. Abstr., 35, 2535 (1941).

57. Krebs, H. A., Biochem. J., 34, 775 (1940).

58. Krebs, H. A., and Eggleston, L. V., Biochem. J., 34, 442 (1940).

59. Baumann, C. A., and Stare, F. J., J. Biol. Chem., 133, 183 ( 1940 ).

60. Adler, E., v. Euler, H., Günther, G., and Plass, M., Biochem. J., 33, 1028 (1939).

61. Dewan, J. G., Biochem. J., 33, 549 (1939).

62. Krebs, H. A., and Cohen, P. P., Biochem. J., 33, 1895 (1939).

63. Cohen, P. P., unpublished studies.

64. Sober, E. K., and Cohen, P. P., unpublished studies. 



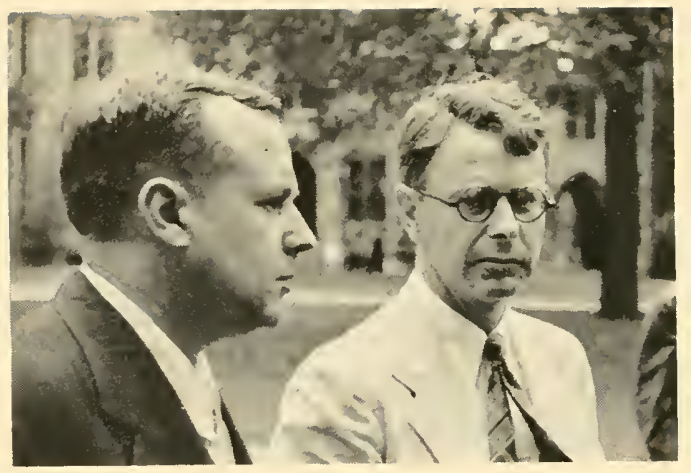

Burk and Elliott

continue the tumor discussion.

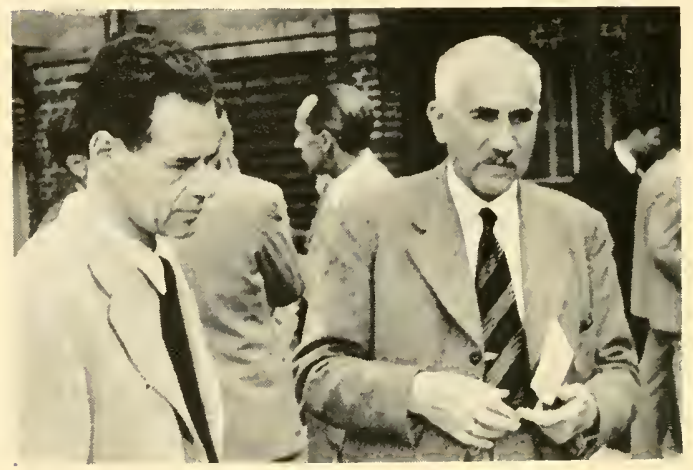

LIPMANN AND MEYERHOF

Why is the Pasteur effect?

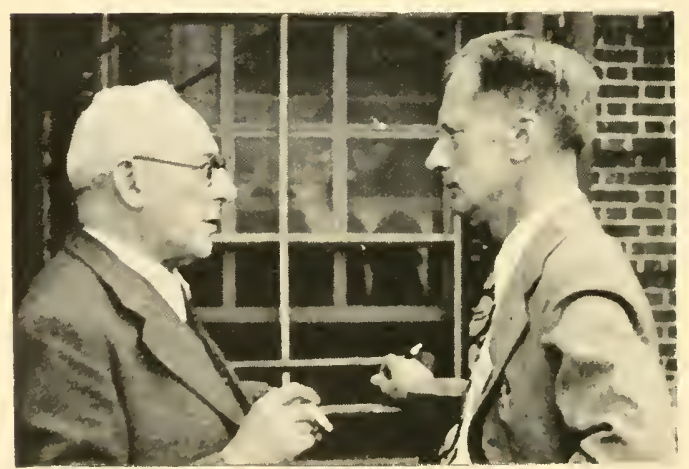

Neuberg axil Cori

Who is consincing whom?

DISCUSSIONS NOT ON THE AGENDA 


\title{
Discussion on Tumor Respiration
}

\author{
C. A. BAUMANN, University of Wisconsin, Chairman
}

\section{CHARACTERISTICS OF TUMOR RESPIRATION}

\author{
K. A. C. ELLIOTT
}

Institute of the Pennsylvania Hospital, Philadelphia

Having been out of the cancer research field for some time, I am not in a position to discuss many recent developments. But it may be useful to start this discussion with a résumé of the generalizations and theories concerning the metabolism of cancer tissue that have been proposed from time to time. My remarks may appear somewhat pessimistic, for it does not seem to me that any definite and peculiar characteristic of tumor metabolism has been proved. However, later speakers who are familiar with more modern work will perhaps strike a more optimistic note.

Warburg's First Theory.-As is well known, Warburg and his school discovered that, whereas slices of a number of normal tissues produce lactic acid from glucose or glycogen rapidly in the absence of oxygen (anaerobic glycolysis), cancer tissue slices show an unusual ability to continue production of lactic acid from glucose in the presence of oxygen (aerobic glycolysis). In his early studies Warburg happened to study tumors which showed very low oxygen uptake rates. He therefore concluded that in cancer tissue the respiratory mechanism was impaired and that glycolysis took its place as a means for producing energy. Later, however, Warburg observed, and emphasized, the fact that the oxygen uptake rate of most tumor tissues under good conditions is not usually lower than that of many normal tissues.

Warburg's Second Theory.-Warburg concluded that since the respiration rate of cancer tissue might be normal while rapid aerobic glycolysis continued, there must be something wrong with the type of respiration in the tumors. Some sort of damage to the respiratory mechanism must have occurred in the production of tumor cells which caused a loss of efficiency of respiration in suppressing glycolysis. High anaerobic glycolysis appeared to be a general property of growing or multiplying tissues, since it was found in embryo tissue and testis, but in these normal tissues glycolysis was largely abolished when respiration occurred, that is, in the presence of oxygen. In 
the preface to the English edition of The Metabolism of Tumors (1), which should be consulted for details of Warburg's work on tumors, Warburg stated that "interference with the respiration in growing cells is, from the standpoint of the physiology of metabolism, the cause of tumors. If the respiration of a growing cell is disturbed, as a rule the cell dies. If it does not die, a tumor cell results. This is no theory, but a comprehensive summary of all the measurements at present available."

At present, however, the idea that respiration itself rather than the presence of oxygen inhibits glycolysis is frequently questioned. High anaerobic glycolysis is not a property of growing tissue alone, since it is found, for instance, in adult brain and in glycogen-rich livers. More important, the high aerobic glycolysis is not found in tumors alone. Warburg himself showed that mammalian retina glycolyzes very rapidly, aerobically as well as anaerobically, though he considered that some sort of damage occurred to this delicate tissue in preparing it for in vitro work. György and co-workers (2) and Dickens and Weil-Malherbe (3) found high aerobic glycolysis in kidney medulla, and the latter authors (4) have recently found the same for jejunal mucous membrane. Testis and several other non-cancerous tissues are now known to show a moderately high aerobic glycolysis, and my co-workers and I have found that a fairly high rate of aerobic glycolysis occurs during the first few minutes of an experiment with various other normal tissues. Murphy and Hawkins (5), on the other hand, reported little or no aerobic glycolysis with some spontaneous tumors occurring in mice. Concerning the normal tissues which show continuous aerobic glycolysis, that is, an "anaerobic type of metabolism" by which energy is produced by the anaerobic method even in the presence of oxygen, Dickens (3) considered that "the cause of an anaerobic type of metabolism is in all such cases merely a disparity between blood supply, i.e., oxygen supply, and energy requirements in vivo." Consideration of aerobic glycolysis as opposed to anaerobic glycolysis does not enter into the following theory of Dickens.

Dickens' Theory.-Dickens and Simer (6) arranged the normal tissues into three groups. One group, which included kidney cortex and liver, showed little anaerobic glycolysis and respired with a rather low respiratory quotient and so apparently metabolized little carbohydrate. Another group, including brain, retina, chorion, and embryo tissue, showed high anaerobic glycolysis and a respiratory quotient of unity in glucose-containing medium. These tissues appar- 
ently respired at the expense of carbohydrate, and in the absence of oxygen the carbohydrate metabolism led to lactate accumulation. A third group, including spleen, testis, and submaxillary gland, formed an intermediate group. But cancer tissue seemed to constitute a separate class, having a high glycolysis and a low respiratory quotient. It was concluded that in cancer tissue the mechanism for starting carbohydrate metabolism is present, even over-developed, but the mechanism for oxidizing the split products of carbohydrate is lacking.

However, Dr. Baker and I (7) found specimens of tumors which respired with respiratory quotient values ranging up to unity and pointed out that other workers, including Dickens himself, had found similar high values. On the other hand, we found values of somewhat less than unity for retina and brain. We concluded therefore, that Dickens' generalization was not valid." Dickens himself, with Weil-Malherbe (4), has now found that normal jejunal mucosa, a rapidly respiring tissue, has a low respiratory quotient with high aerobic and anaerobic glycolysis. A similar type of metabolism was found with synovial membrane by Bywaters (8). Dickens has therefore abandoned his generalization, since these are normal tissues showing the behavior that was believed to be specific for tumors.

It thus appears that no characteristic of metabolism is truly specific for cancer tissue. Nevertheless, most cancer tissues show only a moderately high respiration rate, a moderately low respiratory quotient, and a definitely high sustained aerobic and anaerobic glycolysis, whereas few normal tissues show all these characteristics together. Orr and Stickland (9) very recently found that tumors occurring in the livers of rats fed butter yellow possess the power, as do most cancer tissues, to form lactate from glucose, anaerobically and aerobically, and differ distinctly in this respect from noncancerous liver tissue. Dr. Dean Burk [see below, page 242] has

* Dickens at first defended his theory by attacking our value for the respiratory quotient of brain slices, while ignoring the rest and ridiculing "a complicated theory of salt effects" which did not appear in our paper. Our values for the respiratory quotient of brain slices were supposed to disagree with others in the literature, but careful study showed that objections could be raised to all the results cited, except perhaps Dickens' own. Varying results could be due to the fact that early aerobic glycolysis by brain liberates carbon dioxide from the medium, and this must be carefully controlled if incorrectly high or low respiratory quotient values are to be avoided. Observing this precaution, we ourselves later obtained slightly higher values (18). Our figure for retina has been confirmed by Dixon (19). 
expressed the view that, even as the histology of cancer is sufficiently characteristic to enable a pathologist to recognize the tissue as cancer usually, but not always, so also the experienced student of tissue metabolism could almost always state correctly what is or is not cancer tissue from results of metabolic measurements on tissue slices.

Impaired Respiration Mechanisms.-Warburg's idea that the respiration of cancer tissue is in some manner deranged may still be true, and the idea has inspired a number of studies of individual respiration mechanisms in cancer tissue. Dr. Benoy, Dr. Baker, and I (10) found the succinic oxidase system inactive in slices of certain tumors, and Dr. Greig and I (11) showed that most tumor suspensions tested were low in succinic dehydrogenase and the cytochromecytochrome oxidase systems. I (12) found that tumor breis added to liver or other tissue breis (except heart) rapidly destroyed the succinic oxidase system of the liver or other tissues. Stotz (13) and Potter and DuBois (14) found low cytochrome $c$ content in a number of cancer tissues. Banga (15) found that certain tumors are scarcely able to reduce added oxalacetate; this and the lack of succinic dehydrogenase would indicate impaired catalysis by the mechanism postulated by Szent-Györgyi.

However, Dr. Greig and I found some tumors with a fair amount of succinic dehydrogenase and some normal tissues with little. Some normal tissues, especially pancreas, and also commercial trypsin, would inhibit liver succinoxidase; the inhibition might be a purely in vitro effect and auto-digestion might partly account for low succinoxidase values found for tumor tissues. Potter and DuBois found at least one normal tissue (lung) with as low a cytochrome $c$ content as tumor tissue, and Breusch (16) found that the rate of oxalacetate reduction was negligible also with some normal tissues, namely spleen, lung, placenta, and peripheral nerves.

Dr. Baker and I (17) found that the effects of a number of dyes on the metabolism of tumor tissue were different from their effects on any of the normal tissues tested. Nothing further has come of these observations.

Altogether the various results suggest that cancer tissue tends to differ from normal tissues in its respiratory mechanisms, but no very well-defined difference has yet been disclosed.

\section{REFERENCES}

1. Warburg, O., The Metabolism of Tumors, translated by F. Dickens (Richard Smith, Inc., New York, 1930).

2. György, P., Keller, W., and Brehne, T., Biochem. Z., 200, 356 (1928). 
3. Dickens, F., and Weil-Malmerbe, H., Biochem. J., 30, 659 (1936).

4. Dickens, F., and Weil-Malherbe, H., Biochem. J., 35, 7 (1941).

5. Murphy, J. B., and Hawkins, J. A., J. Gen. Physiol., 8, 115 ( 1925 ).

6. Dickens, F., and Simer, F., Biochem. J., 24, 1301 (1930); 25, 985 (1931).

7. Elliott, K. A. C., and Baker, Z., Biochem. J., 29, 2433 (1935).

8. Bywaters, E. G. L., J. Path. Bact., 44, 247 ( 1937 ).

9. Orr, J. W., and Stickland, L. H., Biochem. J., 35, 479 ( 1941 ).

10. Elliott, K. A. C., Benoy, M. P., and Baker, Z., Biochem. J., 29, 1937 (1935).

11. Eliıott, K. A. C., and Greig, M. E., Biochem. J., 32, 1407 (1938).

12. Elliott, K. A. C., Biochem. J., 34, 1134 (1940).

13. Sтотz, E., J. Biol. Chem., 131, 555 (1939).

14. Potter, V. R., and DuBors, K. P., J. Biol. Chem., 140, cii (1941).

15. Banga, I., Z. physiol. Chem., 244, 130 (1936).

16. Breusch, F. L., Biochem. J., 33, 1757 (1939).

17. Elliotr, K. A. C., and Baker, Z., Biochem. J., 29, 2396 (1935).

18. Elliott, K. A. C., Greig, M. E., and Benoy, M. P., Biochem. J., 31, 1003 (1937).

19. Drxon, M., Biochem. J., 31, 924 ( 1937 ).

\title{
PHOSPHORYLATION THEORIES AND TUMOR METABOLISM
}

\author{
VAN R. POTTER \\ McArdle Memorial Laboratory, University of Wisconsin
}

I should like to submit briefly for your consideration a working hypothesis concerning the metabolism of tumor tissue. This hypothesis is grounded in the Embden-Meyerhof scheme of carbohydrate breakdown, the Warburg descriptions of tumor metabolism and the concept of phosphate energy transfer recently put forth by Johnson. It is difficult if not impossible actually to prove such theories, and hence one must be satisfied with data that do not prove but are merely compatible with the given concept. Only after a great mass of circumstantial evidence has accumulated can we begin to have confidence in the theory. During the accumulation of these data the hypothesis is necessarily modified in the light of incompatible data. It is such incompatible data which probably will soon be brought to bear upon the hypothesis I am about to present.

According to this hypothesis, tumor tissue uses its adenosine triphosphate (ATP) reservoir for but two main purposes, growth and glucose phosphorylation, in contrast with most other tissues, which in addition have function and thus do work and presumably split ATP in doing it. Since the growth stimulus is ever present in tumor tissue, inorganic phosphate is released in large enough quantities to permit rapid glycolysis (in the sense of carbohydrate cleavage), 
yet the growth does not deplete the ATP to such an extent that glucose cannot be phosphorylated and hence glycolyzed. In other tissues glycolysis is slowed down either by a depletion of inorganic phosphate, as in resting tissue, or by a depletion of ATP, as in dying tissue. (Liver and kidney under anaerobic conditions deplete ATP by tending to maintain function and are hence unable to phosphorylate glucose; therefore glycolysis stops and death occurs.) In the tumor tissue the glycolytic rate is so rapid in relation to the oxidative mechanisms that lactate accumulates. The oxygen uptake is limited by the amount of the oxidative enzymes present, but since there is an excess of substrates, the $\mathrm{Q}_{0_{2}}$ is higher than might be expected on the basis of the $\mathrm{Q}_{\mathrm{O}_{2}}$ of normal tissue, in which the oxidative enzymes are present in excess and the $\mathrm{Q}_{\mathrm{O}_{2}}$ is limited by the amount of substrate furnished by glycolysis. In the tumor tissue the growth process outpaces the synthesis of the oxidative enzymes, and the latter become diluted as compared with their concentration in other active tissues. From this it would follow that growth may not require as high a level of oxidative enzymes as does function.

Our experimental results are being reported elsewhere. At this point it may be said that one component of the oxidative mechanism, namely, cytochrome $c$, appears to have been established as definitely lower in the various types of tumor tissue than in normal tissues. Assays on the succinoxidase system are at present being carried out. Preliminary experiments with rapidly growing liver support the idea that growth outpaces the synthesis of the oxidative enzymes temporarily in this tissue. 


\section{ON THE SPECIFICITY OF GLYCOLYSIS IN MALIGNANT}

\section{LIVER TUMORS AS COMPARED WITH HOMOLOGOUS}

\section{ADULT OR GROWING LIVER TISSUES ${ }^{*}$}

\section{DEAN BURK \\ National Cancer Institute, National Institute of Health, U. S. Public Health Service, and Cornell University Medical College}

In this discussion I wish to focus attention upon one particular aspect of tumor and growth metabolism that is simple but farreaching in implication. It is a problem more of comparative biochemistry than of intermediate metabolism proper, and concerns the origin of tumor metabolism. The question I wish to pose, and hope to succeed in answering here, is whether the large glycolysis of tumors is necessarily an expression and requirement of their extensive and usually rapid growth. It has been widely held, since the middle period of Warburg's tumor work (1925), that growing tissues in general have a high anaerobic (and sometimes aerobic) glycolytic activity. This very active metabolism has in turn been attributed to extra and special metabolic requirements of the growth process. If it could be found that certain growing tissues do not exhibit marked glycolysis, then it might well be said that the glycolysis of tumors is not necessarily a consequence merely of extensive growth, but that it has a more specific and characteristic significance for tumor metabolism than has been recognized or acknowledged.

Before presenting data bearing directly on the foregoing question, a related aspect of the problem of the origin of tumor glycolysis, and of suitable criteria for ascertaining significant differences between normal and tumor metabolism, should be discussed by way of background (cf. also ref. 17). In recent years Berenblum, Chain, and Heatley (1) have made the claim that "valid comparisons can only be made between any particular tumor and the normal tissue from which it is derived" (1a, p. 370). This emphatic assertion, which I believe it is very desirable to contravert at this early stage in its possible development, is surely dogmatic and arbitrary to say the least, for there are many valuable comparisons to be drawn between tumor materials and adult tissues widely separated from them embryologically, as well as between tumors and tissues as closely homologous as possible. I for one would not undertake to say which type of comparison would, in fact, be the more profitable in the long run, let alone advocate the exclusion of either one. Certainly

* For much valuable help in the preparation of this manuscript I am greatly indebted to Miss Juliet M. Spangler, Senior Cancer Aide. 
both types of comparison, involving non-homologous as well as homologous contrasts and similarities, must be made.

In furtherance of their position, Berenblum, Chain, and Heatley advance the view that "the tumors which have hitherto been found to have a glycolyzing type of metabolism associated with a low R.Q. possess these properties in virtue of their origin from normal tissues which also possessed these metabolic characters" (1d, p. 138). In experimental support of this view they reported, following Crabtree's earlier measurements showing that whole skin undergoes little alteration of metabolism when it becomes papillomatous, that normal skin epithelium and Shope papilloma of the domestic rabbit also possess essentially the same quantitative metabolism in regard to aerobic and anaerobic glycolysis, respiration and respiratory quotient. Unfortunately these data, although interesting enough in themselves, have no great bearing on the really pertinent problem as to the difference (or similarity) between a definitely malignant tumor and a closely homologous normal tissue; indeed the supporting experiments are themselves somewhat unsatisfactory, being difficult to analyze because of the unorthodox technical method employed and the fact that $Q$ values were based on nucleic acidphosphorus content instead of on dry weight. Certainly there are advantages in the use of the nucleic acid-phosphorus criterion, but it is unfortunate that the dry weight values were not at least reported so that the reader could make $Q$ value comparisons by the standard methods and check, in particular, the bare and doubtful statement $(\mathrm{lb}, \mathrm{le})$ that the normal skin epithelium and Shope papilloma metabolic values were "very similar to those for many skin carcinomas quoted in the literature." (By certain inferences, the anaerobic glycolysis of the skin epithelium and papilloma studied would appear to have been at most $Q^{N_{2}}{ }_{A}=1$ to 3, or quite low for the usual malignant tumor.) The two criticisms of the unorthodox (however correct) procedures employed are admittedly minor as compared with the fact that the Shope papilloma, as such, is not malignant, nor was it so described.

Table 1, now presented for discussion, provides, in regard to primary rat liver tumors, not merely one but several types of homologous tissue, including adult normal liver and two types of growing liver, regenerating and embryonic. These materials will provide, I believe, as pertinent cases as are yet available for the comparison of a malignant tumor with an homologous, in fact identical, tissue of origin (liver). 
The data in Table 1 are the result of the collaboration of many investigators, as indicated in the footnotes, and have been or are being detailed elsewhere under respective authorships. They are brought together here for the purpose of a broad and unified interpretation and discussion at this meeting.

\section{Adult Liver}

The azo dye tumors reported upon in the table offer an excellent opportunity to determine not only whether their metabolism is different from the tissue of origin but also, if it is, to ascertain at what stage or stages of tumor development the altered metabolism appears. The hepatomas, adenocarcinomas, metastases therefrom, and necrotic material thereof, obtained from rats fed butter yellow, and also the mouse tumor transplant derived originally from $o$-aminoazotoluene feeding, all show the high anaerobic glycolysis and low or intermediate respiratory quotient characteristic of malignant tumors, and a considerably increased aerobic glycolysis as compared with either normal rat or mouse liver. The respiration is not changed significantly. There is a definite but relatively small anaerobic lactic acid formation in "pretcancerous," cirrhotic liver as compared with normal liver; there is likewise a slight but quite definite increase in "normal" lobes adjacent to tumor-bearing lobes and in livers of rats protected against tumor formation and extensive liver damage by butter yellow feeding. The aerobic glycolysis increases rather abruptly, essentially at the onset of gross tumor formation, and certainly more abruptly than in the case of the anaerobic glycolysis, where there is a small, but perfectly definite, several-fold increase in the pre-cancerous liver as compared with the normal. The results on tumor in Table 1 in good part confirm and extend the well-known results of Nakatani, Nakano, and Ohara (10), who obtained, in fact, relatively more pre-cancerous change in anaerobic glycolysis, though likewise none in the aerobic. For comparison with absolute and not relative $Q$ values, it is necessary to reduce the Japanese values by four-to five-tenths to put them on an initial dry weight basis corresponding to those in Table 1.

Orr and Stickland (13, and previous preliminary communications) reported, contrary to the results of Nakatani et al. and our own, that they did not observe any change in the glycolytic metabolism of liver tissue in the pre-cancerous stage of butter yellow treatment. For the basis of their comparisons, however, they reported that their normal livers yielded anaerobic glycolysis $Q$ values of 2-16 
(presumably 1-8-12, if corrected to initial dry weights). These results for normal liver, with the possible exception of some very early work of Rosenthal (14), are practically unique in the literature of liver metabolism. In our own experiments, Nakatani $e t$ al., and (so far as I know) essentially all others, anaerobic glycolysis $Q$ values of more than 2 (initial dry weight basis) have never been consistently reported. In our experience with many hundreds of normal rat livers under a great variety of dietary conditions, $Q^{\mathrm{N} 2}{ }_{\mathrm{A}}$ values of 1 or considerably less were regularly obtained in rats weighing over 50 grams (using the ordinary manometric methods, and with varying periods of oxygenation between the killing of the rat and the establishment of anaerobiosis). Without attempting to account at this

\section{Table 1.-A comparison of the metabolism of various rat liver tumors with various homologous liver tissues (normal, embryonic, aged, cirrhotic, regenerating)}

\begin{tabular}{|c|c|c|c|c|c|c|c|}
\hline Tissue & $\begin{array}{c}\text { Rat } \\
\text { Weight } \\
\text { (grams) }\end{array}$ & $\mathrm{Q}^{\mathrm{O}_{2} \mathrm{~A}}$ & $\mathrm{QO}_{2}$ & R.Q. & $\mathbf{Q}^{\mathrm{N}_{2}} \mathrm{~A}$ & M.O. & $\mathbf{U}$ \\
\hline Average Normal Liver (2-24 mos.) & $50-350$ & 1.5 & 6.0 & 0.70 & 1.0 & -0.3 & -11.0 \\
\hline $\begin{array}{l}\text { "Butter Yellow" Liver } \dagger \\
\text { "Normal," yeast protected } \\
\text { "Normal" lobe, adjacent to tu- } \\
\text { mor lobe } \\
\text { Cirrhotic } \\
\text { Necrotic tumor } \\
\text { (Mouse transplant } \ddagger \text { ) } \\
\text { Metastases (to omentum and } \\
\text { mesentery) } \\
\text { Adenocarcinoma-hepatoma } \\
\text { Hepatoma }\end{array}$ & $75-250$ & $\begin{array}{l}1.6 \\
1.3 \\
1.5 \\
3.9 \\
2.1 \\
\\
2.5 \\
3.3 \\
6.0\end{array}$ & $\begin{array}{l}4.9 \\
7.7 \\
6.5 \\
4.2 \\
4.7 \\
5.0 \\
7.3 \\
6.4\end{array}$ & $\begin{array}{l}0.98 \\
0.82 \\
0.82 \\
0.77 \\
0.66 \\
\\
0.83 \\
0.84 \\
0.87\end{array}$ & $\begin{array}{r}2.1 \\
\\
2.3 \\
3.1 \\
6.0 \\
7.7 \\
\\
8.7 \\
10.0 \\
12.1\end{array}$ & $\begin{array}{l}0.3 \\
\\
0.4 \\
0.7 \\
1.5 \\
1.2 \\
\\
3.7 \\
2.8 \\
2.9\end{array}$ & $\begin{array}{l}-7.7 \\
-13.1 \\
-9.9 \\
-2.4 \\
-1.7 \\
-1.3 \\
-4.6 \\
-0.7\end{array}$ \\
\hline
\end{tabular}

* $\mathrm{Q}$ values based on initial dry weights (original data of Tamiya (16) based on final dry weights and here factored by 50 per cent to reduce to approximate initial dry weight; cf. refs 5 and $8 \mathrm{c}$ ). $\mathrm{QO}_{2}, \mathrm{Q}^{\mathrm{N}_{2}} \mathrm{a}$ and $\mathrm{Q}^{\mathrm{O}_{2}} \mathrm{~A}=\mathrm{mm} \cdot{ }^{3}$ oxygen consumption, anaerobic and aerobic acid production $/ \mathrm{mg}$. initial dry weight of tissue per hr.; R.Q.= respiratory quotient $=\mathrm{QO}_{2} / \mathrm{Q}_{\mathrm{CO}_{2}} ;$ M.O.Q. = Meyerhof oxidation quotient $=3\left(\mathrm{Q}^{\mathrm{N}_{\mathrm{A}}}-\mathrm{Q}^{\mathrm{O}_{2}} \mathrm{~A}\right) / \mathrm{Q}_{2}$; $\mathrm{U}=$ fermentation excess $=\mathrm{Q}^{\mathrm{N}_{2}} \mathrm{~A}-2 \mathrm{Q}_{2}$.

† Sample of average data taken with O. K. Behrens and K. Sugiura (cf. ref. 4 et seq.). Up to 30 specimens of each tissue type examined metabolically. Rats fed 0.06 per cent $p$-dimethylaminoazobenzene (butter yellow) for 150-200 days on brown rice-carrot diet. "Yeast-protected" with 5-15 per cent added dried brewer's yeast.

$\$ 33 \mathrm{~d}$ generation, subcutaneously transplanted, o-aminoazotoluene-induced liver carcinoma 1 in dba strain. Mouse obtained from Dr. H. B. Andervont through Dr P. M. West; liver of same animal: $\mathrm{Q}^{\mathrm{N}_{2}} \mathrm{~A}=0.8 ; \mathrm{Q}^{\mathrm{O}_{2}} \mathrm{~A}=1.0$. 
Table 1.-continued

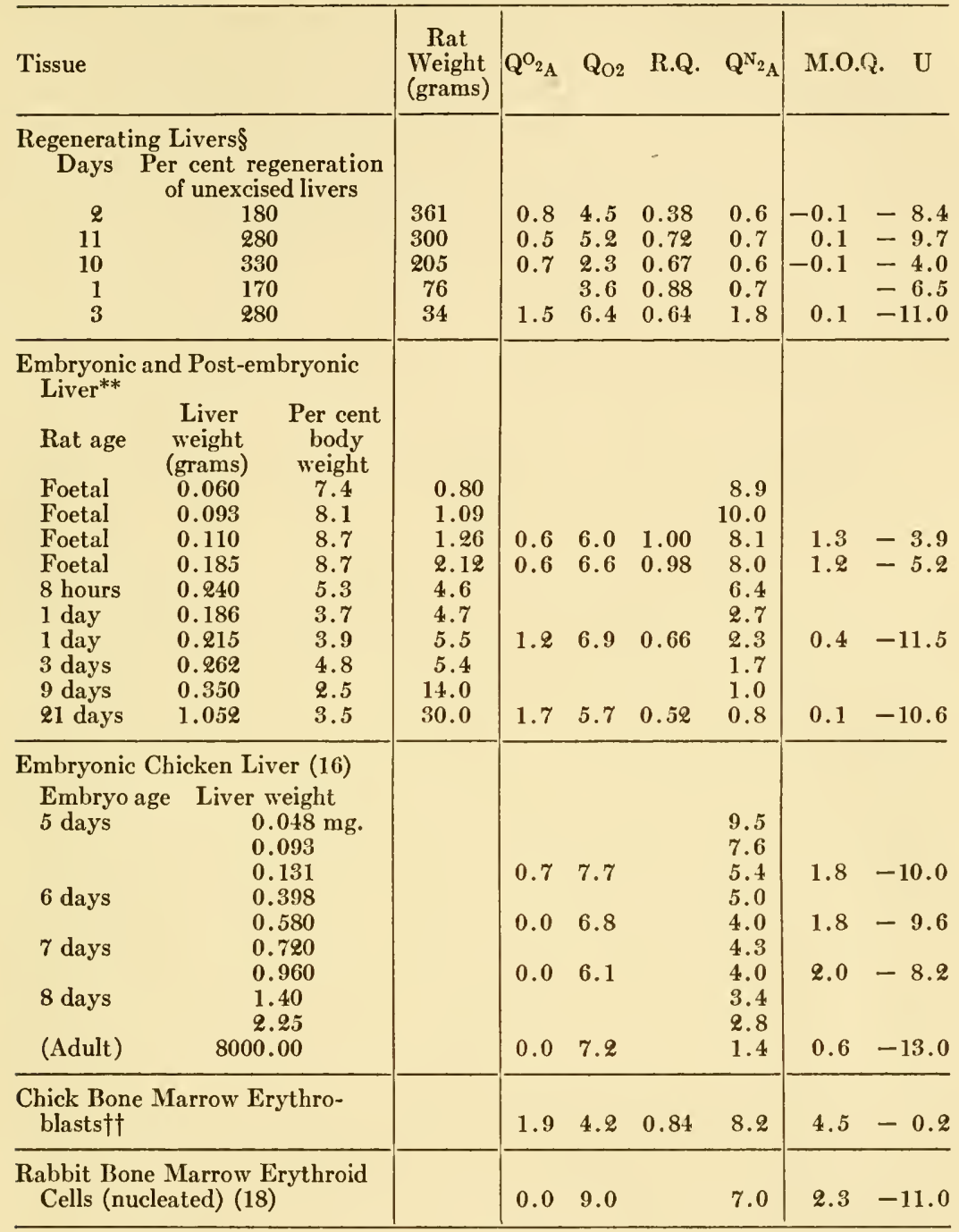

\$ Sample of data taken with J. Blanchard, C. Povolny, J. Norris, and J. Saxton (12). Rats 10-600 days old (15-400 grams body wt.); regeneration 1-11 days after original 65 per cent hepatectomy (left and median lobes extirpated).

** Data taken with J. Norris $(11,12)$.

†† Average of data taken with H. Sprince, E. A. Kabat, and J. Furth on chickens treated with acetylphenylhydrazine to produce hyperplastic (not leukotic) bone marrow, with some leukogenic cells but mainly erythroblasts. To be published. 
time for the exceptional results of Orr and Stickland with normal livers, it can very definitely be stated on the basis of our results and those of Nakatani et al., that there is a one or more fold increase in the anaerobic glycolytic metabolism (and likewise glucolytic, for those interested in this distinction) of liver tissue in the pre-cancerous stage of butter yellow treatment. That this increase, unless due to a rather inconceivable difference in rat strains, "must have been due to chance," as proposed by Orr and Stickland (13, p. 486), is out of the question, and we prefer the alternate view that the very unusual magnitude and spread of normal liver values obtained by Orr and Stickland have served to confuse rather than to clarify the results they observed with the pre-cancerous livers. A second and regular point of difference in the pre-cancerous livers observed by us was that the initial rate of glycolysis was better maintained over a period of several hours, whereas in the normal livers the $\mathrm{Q}^{\mathrm{N} 2}{ }_{\mathrm{A}}$ values dropped to zero or a few tenths in the course of an hour or two, and this relative effect was even more striking when the $Q$ values were based on chemically measured lactic acid rather than on manometric acid production. It is conceivable, in the absence of information to the contrary, that the Orr and Stickland determinations on normal liver do not refer to measurements over sustained periods of time (hours), and that in some way the high normal values reported by them involve incidental aspects of initial or preparatory phases of technique, in some measure connected, to be sure, with the glycogen content of livers, as they demonstrated; I hesitate to suggest explicitly the trite explanation of extensive damage, but evidently some factor is operating to give them profoundly atypical (this is not to say incorrect) values for normal liver that certainly make comparisons with other kinds of liver material difficult.

Regardless of the foregoing discrepancy of result in regard to pre-cancerous livers, all investigators agree that lactic acid formation from glucose by malignant tumors induced by butter yellow is strikingly different from that of normal liver and definitely or considerably increased over any form of pre-cancerous liver, and that the same is true in less marked degree with respect to aerobic lactic acid formation. In general, the anaerobic and aerobic lactic acid productions by these hepatomas are, on an absolute basis, intermediate between those of most rat, human, and chicken malignant tumors, on the one hand, studied by Warburg and many others afterward, and, on the other hand, those of certain mouse tumors studied originally by Murphy and Hawkins, and by Crabtree and 
Cramer and others later. The fermentation excess, $U$, is in fact not positive but zero or slightly negative, and the Meyerhof oxidation quotient is nearer 3 than 6 .

In regard to the low or intermediate respiratory quotient of the azo dye tumors of Table 1, I might comment, in view of the frank discussion and expression of personal opinion desired here, that in the recent discussions in Nature on the metabolism of tumors by Dickens (7), Boyland (2), Berenblum, Chain, and Heatley (1b), and Dickens and Weil-Malherbe (8b), I agree in general with the comments of Dickens and disagree with the other commentators where they take exception, for in my judgment they fail to introduce the proper quantitative perspective. However, I do not feel that Dickens has been correct, during the past decade, in his view that "cancer tissue has a respiratory quotient indicating that the oxidation of carbohydrate is abnormal" (7, p. 512). I prefer to regard the low or intermediate respiratory quotient exhibited by the majority of malignant tumors as being unchanged from the similar low or intermediate respiratory quotient values of the great majority of normal adult tissues, those, in fact, cited by him over a decade ago. So why refer to them as "abnormal"? Why not consider them as simply unaltered? It is the glycolytic capacity, not the respiratory quotient, of tumors which by and large has changed or is "abnormal" or different from normal adult tissue; it is in most growing normal tissues that the respiratory quotient has tended to rise to or attain unity, and the oxidation of carbohydrate to become relatively more pronounced, and also the capacity for glycolysis (mainly anaerobic). General confusion on these matters has led some, including Dr. Elliott, in his intentionally pessimistic comments this morning, to suggest, with reference to the very recent paper of Dickens and Weil-Malherbe (8c) that the high glycolysis and low respiratory quotient found by them for jejunum mucosa put the metabolism of this tissue into the class of malignant tumor metabolism; but the very high absolute $Q$ value for respiration (about equal to the high anaerobic glycolysis $Q$ value) and the absence of a Pasteur effect make this designation, in my opinion, quite impossible. Likewise, if not one or two but a sufficient number of metabolic criteria (absolute and relative) are considered, it is impossible to agree with the recent tendency (1a, and possibly 7) to regard cartilage (data, 6, 8a) and synovial membrane (data, 6b) as, like the alleged skin epithelium (1), providing rather good examples of normal tissues with "malignant tumor metabolism," even after appropriate correction for inert ma- 
terials in these tissues and, I must add, also in the tumors taken for comparison! I know of no normal tissue whose metabolism, fully regarded, need as yet be confused with that of malignant tumors." Further background for the foregoing interpretation of quite recent data is detailed elsewhere $(3,4,5)$.

\section{Growing Liver}

One type of homologous tissue has been presented, but it might still be argued that the normal liver, although homologous, was not a growing tissue and not as comparable with liver tumor as might

* I may reiterate a statement I have already made on many occasions, namely, that I believe that the metabolic diagnosis of malignant tumor as compared with normal tissue may be correlated with pathologic diagnosis in well over 95 per cent of tested cases (and, I venture to say, as yet untested cases), upon due consideration of the absolute as well as the relative magnitudes of, first and foremost, anaerobic glycolysis $(8-20 \pm)$ and of respiratory quotient $(0.75-0.9 \pm)$; secondly, respiration $(2-10 \pm)$, and aerobic glycolysis $(0-15 \pm)$; and thirdly the derived quotients, absolute Pasteur effect $(8-15 \pm)$, Meyerhof oxidation quotient (M.O.Q.) ( $3-6 \pm$ ), fermentation excess (U) ( -5 to + $25 \pm$ ) etc., ( $Q$ values based on initial dry weights); and fourthly quite possibly the new criterion developed by Salter et al. (15) in regard to separation of certain tumors from their homologues on the basis of differential oxidation of glucose and succinate. Non-tumor tissues can be excluded from malignant tumor tissue designation by one or more of these metabolic criteria; thus, to consider previously debated cases: for the kidney medulla, too high an R.Q.; cartilage, synovial membrane, and (presumably) skin epithelium, too low an anaerobic glycolysis $\left(\mathrm{Q}^{\mathrm{N}^{2}}{ }_{\mathrm{A}}\right)$ or respiration $\left(\mathrm{Q}_{\circ 2}\right)$ even with reasonable correction for intercellular substance and inert components; retina and jejunal mucosa, too high a respiration and in the latter case also M.O.Q. $=0$ (no Pasteur effect).

The recent discussion, pro or con ( $1 \mathrm{c}, 1 \mathrm{~d}, 7,2,1 \mathrm{~b}, 8 \mathrm{~b}$ ), and empliasis laid, on aerobic glycolysis would in my opinion be much better transferred to anaerobic glycolysis, which without exception, to my knowledge, is always considerable in malignant tumors. From my point of view aerobic glycolysis is almost invariably merely an expression (consequence) of how much anaerobic glycolysis goes on in relation to how much oxygen consumption is occurring in the particular tissue under examination $(3 b, 4)$. In malignant tumors for example, it can be said that the anaerobic glycolysis values are so high relative to the respiration that the latter is unable to inhibit completely the glycolysis under aerobic conditions, even with extensive operation of the Pasteur effect (M.O.Q. $=3-6$ ) (5); the aerobic glycolysis thus resulting is a quantity dependent upon two rather independent functions, oxygen consumption and anaerobic glycolysis. I might add, parenthetically, that most of the aerobic glycolysis values reported as zero in the literature (including the often quoted mouse data of Murphy and Hawkins) are in fact definitely positive due to a methodological error of not correcting the calculations for the fact that the R.Q. is ordinarily definitely less than unity, and hence the aerobic glycolysis greater than otherwise calculated. Unfortunately I cannot go here more deeply into details of elaboration needed to treat adequately the subjects discussed in this footnote and the two sentences that gave rise to it, but shall do so when the butter yellow tumor data summarized in Table 1 are described at length. 
be desired. Two more types of homologous tissue are presented in Table 1 by the data on regenerating liver and embryonic liver, both of which tissues may at certain stages attain growth rates even greater than that of liver tumor. The data on regenerating liver are very striking in that they show, as compared with normal adult liver, no appreciable alteration in any of the metabolic values studied. In the case of the very young rats, the regenerating $Q^{N 2}{ }_{A}$ value is slightly increased, but mainly as a matter of neonatal age rather than of regeneration. Regenerating liver is indeed a remarkable case, demonstrating that tissue growth may take place without appreciable glycolysis, and at the expense, even, of unchanged oxygen consumption; for the growth increase in liver tissue (on a water-free basis) may attain 50 to 100 per cent per day during the most active phases of regeneration at about two to three days after partial hepatectomy, when mitotic figures are most numerous, several being visible on a high power field, or even more than would be found with a butter yellow liver tumor. Orr and Stickland (13) reported that the glycolysis of regenerating liver was not appreciably different from that of the normal livers they examined, but in these data the issue is again confused and rendered indefinite by their exceedingly high and variable normal liver values.

A third type of homologous tissue is the rapidly growing embryonic liver. Tamiya (16) showed over a decade ago that there is a marked rise in the anaerobic glycolysis of chicken embryo livers the younger the embryo and the smaller the liver (at least back to a very early stage or microscopic size). The recent experiments of J. Langdon Norris $(11,12)$ give essentially the same results for embryonic rat livers as for the embryonic chicken livers. Contrary, however, to opinion held since the work of Tamiya (16) and Hawkins (9) that embryonic liver in particular and growing tissues in general show considerable anaerobic glycolysis, the histopathologic sections taken by Norris (illustrated elsewhere, 11, 12) show that the smaller the embryonic livers and the greater the anaerobic glycolysis, the greater and in parallel manner is the extent of haematopoesis, which in the extreme may amount to an estimated 70 to 80 per cent of the liver, involving mainly erythropoietic cells (with some megakaryocytes and myelogenic cells). The metabolism of these nucleated erythropoietic cells has not yet been measured directly, but two comparable types of nucleated erythroid cells are available for comparison, namely, the chick bone marrow erythroblasts produced by acetylphenylhydrazine injections and the normal rabbit bone mar- 
row erythroid cells. In both these cases the anaerobic glycolysis $Q$ values are of the same order of magnitude (7-10) as that of the embryonic livers containing a high percentage of red cells, and the other metabolic values are likewise comparable. Direct measurement of the metabolism of the red cells in the embryonic livers offers considerable difficulty experimentally; moreover, when they are obtained, the measurements might still be somewhat uncertain because of possible secondary effects of the technical methods employed to separate them from the liver. But it is felt that in the light of the two quite comparable cases offered and a considerable background of knowledge regarding the metabolism of blood cells generally, there is no reason to doubt that the rather high glycolytic metabolism of embryonic livers is due to the erythropoietic element and not to the true liver cells. In other words, neither the embryonic liver per se, nor the regenerating liver, nor adult normal liver, nor in fact any healthy liver, growing or otherwise, possesses a noteworthy glycolysis, in contrast with the various malignant hepatomas, where a large glycolytic capacity obtains. The case of Berenblum, Chain, and Heatley (1b) that "when a tumour is compared with the tissue from which it is derived, there are no metabolic characteristic differences or peculiarities between the carbohydrate metabolism of the two" is clearly not valid; nor, for lack of evidence, is the more general contention quoted earlier that tumors glycolyze by virtue of their origin from normal tissues which also possess this metabolic character* (1d, p. 138).

The question whether the increased formation of lactic acid in the hepatic tumors-or in tumors generally-is necessarily an expression or requirement of the growth involved, as is commonly believed, is thus answered in the negative by the experiments briefly described in Table 1. It may be concluded, more generally, that growth does not necessarily require glycolysis, and may on occasion be maintained at the highest levels on an essentially aerobic non-fermentative metabolism.

If it should be asked why malignant tumors possess glycolysis if not because of growth, I would venture the opinion that the glycolysis is better correlated with the more primitive organization or lesser differentiation involved.

Most of the foregoing discussion has been concerned with com-

- I do agree with Berenblum et al.- - and not with Dickens, as already indicated-that the medium-low respiratory quotient of tumors may well be derived from the tissues of origin, in the sense that it remains by and large unchanged in tumors. 
parative biochemistry, not intermediate metabolism. The studies made in recent years by Elliott and by Potter, and especially this year by Salter and collaborators (15), on the deficient (cytochromesuccinate) oxidation systems in many tumors as compared with tissues of varying degrees of homologousness, will undoubtedly lead the way in indicating by what mechanisms, if any, the respiration systems of tumors permit high glycolysis to occur. But that is a story for the future. The paper of Mr. Kensler now to follow, based on his study with Dr. C. P. Rhoads (9a) on the action of butter yellow intermediates (free radicals) in correlating metabolism and carcinogenic action, will present an exceedingly promising pioneer work that opens up an entirely new approach to the connection between tumor metabolism and tumor genesis, an approach that will, I believe, command the admiration of us all.

\section{REFERENCES}

1. Berenblum, I., Chain, E., and Heatley, N. G., (a) Amer. J. Cancer, 38, 367 (1940); (b) Nature, 145, 778 (1940); (c) Ann. Rep. Brit. Emp. Cancer Campaign, 16, 215 (1939); (d) ibid., 17, 135 (1940); (e) Abstr. 3d Inter. Cancer Congress, Atlantic City, p. 127 ( 1939 ).

2. Boyland, E., Nature, 145, 512 (1940).

3. Burk, Dean, (a) Occas. Publ. Amer. Assoc. Adv. Sci., No. 4, 121 (1937); (b) Cold Spring Harbor Symposia on Quantitative Biology, 7, 420 ( 1939).

4. Burk, Dean, Behrens, O. K., and Sugiura, K., Cancer Res., 1, 733 (1941) et seq.

5. Burk, Dean, Sprince, H., Spangler, J. M., Kabat, A. E., Furth, J., and Claude, A., J. National Cancer Institute, 2, 201 ( 1941).

6. Bywaters, E. G. L., (1) Nature, 138, 30 (1936); (b) J. Path. Bact., 44, 247 ( 1937 ).

7. Dickens, F., Nature, 145, 512 (1940).

8. Dickens, F., and Weil-Malmerbe, H., (a) Nature, 138, 125 (1936); (b) ibid., 145, 779 (1940); and (c) Biochem. J., 35, 7 (1941).

9. Hawkins, J. A., J. Gen. Physiol., 9, 771 ( 1926 ).

9a. Kensler, C. J., Dexter, S. O., and Rhoads, C. P., Cancer Res. 2, 1 (1942).

10. Nakatani, M., Nakano, K., and Ohara, Y., Gann, 32, 240 ( 1938 ).

11. NorRis, J. LANGDON, "Metabolism of rat livers, with particular reference to embryonic liver and malignancy," Polk Prize Paper, Cornell University Medical College, Sept., 1941.

12. Nornis, J. L., Blanchard, J., and Povolny, C., "Regeneration of rat liver at different ages and metabolism of embryonic neonatal and regenerating rat liver," Amer. J. Path., Opie Dedication Number (in press).

13. OrR, J. W., and Stickland, L. H., Biochem. J., 35, 479 ( 1941 ).

14. Rosenthal, O., Biochem. Z., 207, 263 (1929).

15. Salter, W. T., Craig, F. N., and Bassett, A. M., Cancer Res., 1, 751 (1941); cf. 1,869 (1941).

16. Tamiya, C., Biochem. Z., 189, 175 (1927).

17. Voegtlin, Carl, Physiol. Rev., 17, 92 (1937).

18. Warren, C., Amer. J. Physiol., 131, 176 (1940). 


\section{THE EFFECTS OF CERTAIN DIAMINES ON ENZYME SYS- TEMS, CORRELATED WITH THE CARCINOGENICITY OF THE PARENT AZO DYES}

\section{J. KENSLER}

Memorial Hospital, New York

In 1935 Hashimoto (1) reported the isolation of acetyl-2-methyl- $p$ phenylenediamine from the urine of rats fed the carcinogen, $o$ aminoazotoluene. Another azo carcinogen, dimethylaminoazobenzene (butter yellow), the metabolism of which has been studied by Stevenson, Dobriner, and Rhoads (2), has also been found to be split in vivo at the azo linkage. The urine of animals fed butter yellow contained aminophenol and $p$-phenylenediamine. The free and acetylated forms of both compounds were found. No dimethyl- $p$ phenylenediamine was isolated, but it may be assumed until evidence to the contrary is presented that it is a precursor of the excreted $p$-phenylenediamine. Orthoamidoazotoluene and dimethylaminoazobenzene are the only carcinogenic azo compounds whose metabolic breakdown has been studied.

A previous report (3) from this laboratory presented evidence that the concentration of diphosphopyridine nucleotide (Coenzyme I) in the livers of rats fed dimethylaminoazobenzene is 60 per cent less than in the livers of rats fed the same basal diet without the carcinogen. This fact suggested that the administered dimethylaminoazobenzene, or some metabolic breakdown product of it, might depress in vitro the activity of a fermenting system from yeast in which diphosphopyridine nucleotide is the limiting factor. Experiment proved the validity of this suggestion. The methods and the results obtained are presented in detail in a report which will appear shortly (4).

In brief, it was found that whereas the original carcinogen, butter yellow, did not inhibit fermentation at all, the isolated derivative of butter yellow, $p$-phenylenediamine, was strongly inhibitory, and dimethyl- $p$-phenylenediamine, the supposed precursor of the simpler compound, had an even more powerful toxic effect.

In view of the results obtained with butter yellow and its breakdown products, it seemed desirable to test on the same fermenting system the effect of chemically related substances. Dr. Leonor Michaelis, who had previously prepared a large number of methyl derivatives of $p$-phenylenediamine as a part of his general study of two-step oxidations, generously provided samples of these compounds for use in this investigation. Their inhibitory effects on the 
rate of fermentation in the yeast system were measured. In Table 1 are presented the results of tests of only those substances of which the carcinogenic potency of the primary azo compound for rat livers has been tested. However, in further tests of the inhibitory effect on the yeast system of seventeen of the methyl derivatives of $p$-phenylenediamine, as well as of those listed, the inhibition was found to correlate closely with the stability of the semiquinone intermediary oxidation product as reported by Michaelis, Schubert, and Granick (5).

In the experiments with the yeast system it was noted, furthermore, that those compounds which were toxic and which formed stable free radicals (semiquinones) were oxidized readily by the yeast apozymase used. The substances which were shown by Michaelis and his associates not to form stable free radicals were not so oxidized. This suggested, therefore, that the stable free radical or some further oxidation product of it was responsible for the inhibition observed.

Among the possible end products of the oxidation that might be responsible for the inhibition are quinone, methylamine, dimethylamine, and formaldehyde. Of these compounds only quinone was found to have significant toxicity. This compound, however, was less than half as toxic as the diamine in equivalent concentrations. Hydrogen peroxide, which can be formed under certain conditions of oxidation, was also proved to be non-toxic. The acetylated $p$-phenylenediamine, which is quite stable and is a metabolite of dimethylaminoazobenzene, was found to be non-toxic.

This inhibition of the diphosphopyridine nucleotide system by the $p$-aromatic diamines made it desirable to test another yeast enzyme system. Similar results were obtained when a carboxylasecocarboxylase system was employed, except for one difference. The addition of reducing agents such as cysteine, glutathione, and ascorbic acid reduced markedly the toxicity of the $p$-aromatic diamines for the diphosphopyridine nucleotide system. In the carboxylasecocarboxylase system, on the other hand, to which the same substances are inhibitory and where there is no complicating catalytic oxidation of the inhibiting agent, the addition of the reducing substances prevents any inhibition at all.

In experiments with both the diphosphopyridine nucleotide system and the cocarboxylase system, where the inhibition by the toxic compounds was large in the presence of low coenzyme concentrations, it was found that the addition of large amounts of coenzyme 


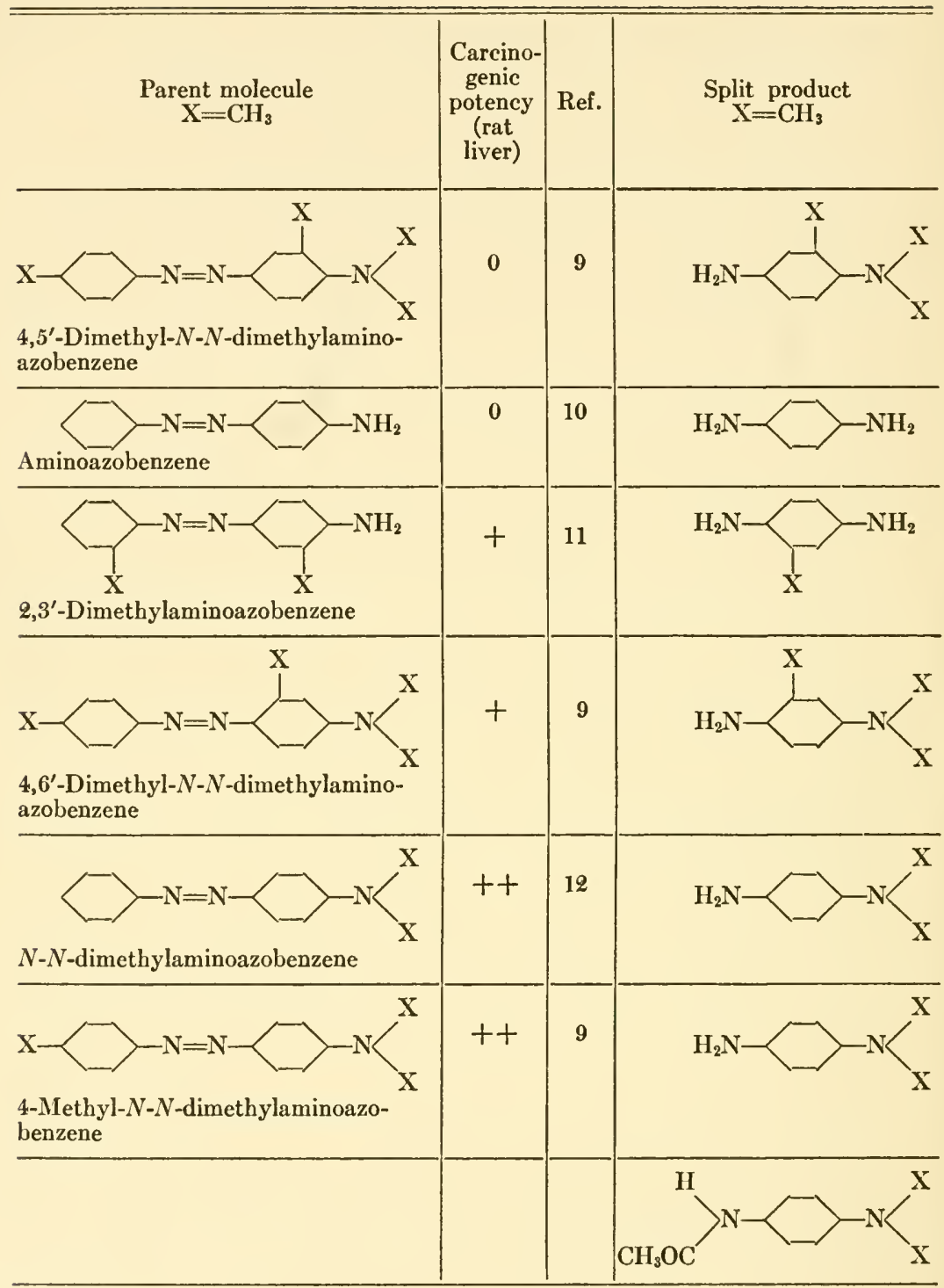

* The table on these facing pages presents the results of tests showing the apparent correlation between the carcinogenic properties of the parent azo compounds and the toxic properties of the $p$-aromatic diamine split product of these molecules. 


\begin{tabular}{|c|c|c|c|c|c|}
\hline \multirow{2}{*}{$\begin{array}{l}\text { Stability } \\
\text { of } \\
\text { free } \\
\text { radical } \\
\text { of split } \\
\text { product }\end{array}$} & \multirow{2}{*}{$\begin{array}{l}\text { Oxidation by } \\
\text { cytochrome- } \\
\text { oxidase } \\
\text { system* } \\
\text { cmm. } \mathrm{O}_{2} \text { per } \\
10 \text { minutes }\end{array}$} & \multicolumn{4}{|c|}{ Percentage Inhibition of Enzyme Systems } \\
\hline & & $\begin{array}{l}\text { diphospho- } \\
\text { pyridine } \\
\text { nucleotide } †\end{array}$ & $\begin{array}{c}\text { cocarboxy- } \\
\text { lase } f\end{array}$ & respiration $\ddagger$ & $\begin{array}{l}\text { oxygen } \\
\text { consump- } \\
\text { tion** }\end{array}$ \\
\hline 5 minutes & 2 & 0 & 9 & 10 & 0 \\
\hline 4-8 hours & 160 & 38 & 51 & 16 & 0 \\
\hline 4-8 hours & 164 & 65 & 67 & 39 & 21 \\
\hline 2 days & 176 & 83 & 75 & 45 & 32 \\
\hline 7 days & 165 & 92 & 81 & 41 & 41 \\
\hline 7 days & 165 & 92 & 81 & 41 & 41 \\
\hline $\begin{array}{c}\text { very } \\
\text { unstable }\end{array}$ & 0 & 2 & 5 & 20 & 4 \\
\hline
\end{tabular}

* M/50 concentration used. No inhibition of the cytochrome-oxidase system with M/50 $p$-phenylenediamine results with any of the split products at concentrations of $5 \times 10^{-4} \mathrm{M}$.

$\dagger$ System: washed yeast; compounds at a concentration of $5 \times 10^{-4} \mathrm{M}$.

$\mp$ System: rat liver slices, 5-hour experiment; compounds at a concentration of $1 \times 10^{-3} \mathrm{M}$.

** System: rat liver brei, 2-hour experiment; compounds at a concentration of $5 \times 10^{-4} \mathrm{M}$. 
(500-1000 micrograms) prevented the inhibition. But when large amounts of coenzyme were added, the inhibition was prevented only in the system in which it was the limiting factor; that is, the addition of large amounts of diphosphopyridine nucleotide maintained activity only in the yeast-fermenting system and did not reduce the toxicity of the diamino compounds in the cocarboxylase system; and, conversely, the addition of large amounts of cocarboxylase maintained activity only in the carboxylase system. These experiments indicate that the inhibition in both systems is a competitive one and further suggest that the action of the diamino compounds is on the protein enzyme component rather than on the coenzyme.

Rat liver cell (slice) and rat liver suspension (brei) oxidations are also inhibited by the $p$-aromatic diamines. The toxicity gradient of these compounds to surviving liver tissue is similar to that observed in the yeast systems. To detect the protection of these rat liver systems by the addition of reducing agents is not practicable, for it was observed that the presence of these diamino compounds in the crude liver enzyme systems catalyzed the oxidation of the reducing agents, cysteine and ascorbic acid. Those diamino compounds that are most toxic act as stronger catalysts of the oxidation of the reducing agents than do the less toxic compounds in the presence of a liver suspension. It was important to establish the fact that the $p$-aromatic diamines are not simply non-specific enzyme poisons. The $d$-amino acid oxidase, tyrosinase, cytochrome oxidase, and acid and alkaline phosphatase enzymes were not inhibited by dimethyl$p$-phenylenediamine in equivalent concentrations $\left(5 \times 10^{-4}\right.$ molar $)$.

In these experiments (see Table 1) the gradations of the toxicity of the diamino split products of methyl derivatives of aminoazobenzene parallel the carcinogenic potency of the parent molecules. But inasmuch as the list of compounds of this series which have been tested for carcinogenic power is small, further animal experiments with other related compounds are needed to determine whether this apparent correlation is a true one. Furthermore, the evidence that the production of $p$-aromatic diamino split products from the parent azo molecule in the liver of the rat is concerned in the resulting production of hepatic cancer is entirely indirect.

Although the mechanism of the inhibition of the protein enzymes in these several systems by the $p$-aromatic diamine is obscure, it appears that the formation of an oxidation product or products of the reduced compounds is essential to secure this effect. The indirect 
evidence available suggests, in addition, that the inhibition may be produced by oxidation of, or combination with, sulfhydryl groups on the protein enzyme. For example, in the diphosphopyridine nucleotide system iodoacetate and alloxan, which are known to react with sulfhydryl groups, also inhibit fermentation. The alloxan inhibition is, like that caused by the $p$-aromatic diamines, a competitive one. Vassel (6) has found that dimethyl-p-phenylenediamine will condense with cysteine in strongly acid solutions in the presence of $\mathrm{Fe}^{+++}$and $\mathrm{ZnCl}_{2}$ to yield a colored (blue) product. In several experiments done in our laboratory we found that in the presence of a liver suspension (brei) an orange product is formed when both dimethyl- $p$-phenylenediamine and cysteine are added. This product is not formed on the addition of either alone.

The work of White (7) has shown that organic sulfur (cystine, methionine) can counteract the growth-inhibiting properties of both the azo and hydrocarbon carcinogens and supports the view that the carcinogen may combine with the sulfhydryl groups of proteins. Further evidence is provided by the studies of L. F. Fieser and his collaborators (8). These concern experiments with the hydrocarbon carcinogens in which an entirely different approach was followed. The results suggest that the action of the hydrocarbon carcinogens may be on an S-S link of a protein and serve to emphasize the need for further information on the mode of action of the $p$-aromatic diamines in catalytically active systems.

\section{REFERENCES}

1. Hashimoto, Y., Gann, 29, 306 ( 1935 ).

2. Stevenson, E. S., Dobriner, K., and Rhoads, C. P., in press.

3. Kensler, C. J., Sugiura, K., and Rhoads, C. P., Science, 91, 623 (1940).

4. Kensler, C. J., and Rhoads, C. P., Journal of Cancer Research, 2, 1 (1942).

5. Michaelis, L., Schubert, M. P., and Granick, S., J. Amer. Chem. Soc., 61, 1981 (1939).

6. Vassel, B., Jour. Biol. Chem., 140, 323 (1941).

7. WhrTe, J., J. Natl. Cancer Inst., 1, 337 (1940).

8. FIESER, L. F., Production of Cancer by Folynuclear Hydrocarbons (University of Pennsylvania Press).

9. NAGAO, N., Gann, 35, 20 (194I).

10. Sasaki, R., and Yosida, T., Virchow's Archiv., 295, 175 ( 1935 ).

11. Yosida, T., Virchow's Archiv., 283, 29 (1932).

12. Kinosits, R., Trans. Soc. Path. Jap., 27, 665 (1937). 


\title{
Discussion on Bacterial Respiration
}

\author{
W. H. PETERSON, University of Wisconsin, Chairman
}

\section{CRITERIA FOR EXPERIMENTS WITH ISOTOPES}

\section{H. G. Wood, Iowa State College:}

The criteria that are to be used in considering the reliability of isotopic work will vary considerably with the type of investigation. For example, in studies with carbon of atomic weight 13 in which one wishes to determine qualitatively whether or not carbon dioxide is fixed in a biological reaction, the criteria are relatively simple. The reaction is simply conducted in an atmosphere containing carbon dioxide in which the content of $\mathrm{C}^{13}$ has been increased artificially above that of carbon found in nature. Carbon in nature contains about 1.1 per cent $\mathrm{C}^{13}$. The carbon dioxide used for a tracer usually contains from 5 to 15 per cent $\mathrm{C}^{13}$, the concentration depending on the method used to obtain the heavy isotope. To determine whether or not carbon dioxide is incorporated into an organic compound, it is necessary only to free the reaction mixture of carbon dioxide, convert the organic compounds to carbon dioxide, and determine its content of $\mathrm{C}^{13}$ in a mass spectrometer. If the $\mathrm{C}^{13}$ content of the reaction mixture is significantly above 1.1 per cent (the natural complement of $\mathrm{C}^{13}$ ), carbon dioxide has been incorporated into organic compounds. This is obviously the case, since there is no other source of carbon than carbon dioxide with a content of $\mathrm{C}^{13}$ above the normal.

The question is, what reliable conclusion can be drawn from such an experiment? Clearly, the only conclusion that can be made is that carbon dioxide is fixed, but no idea is given as to the reaction involved or the compounds concerned. In the early isotopic work such experiments were given undue significance. For example, this type of experiment was conducted by Ruben and Kamen with pigeon liver, Escherichia coli, and a number of other heterotrophic systems and it was found that a small amount of radioactive carbon dioxide was fixed. These experiments have been cited as examples of assimilation of carbon dioxide by heterotrophic organisms. There is no general agreement on a definition of assimilation, but it is certain that to 
many the term implies construction of cell material, enzymes, and perhaps organic compounds involving a carbon chain. Clearly this simple demonstration of carbon dioxide fixation is not a reliable criterion of assimilation so defined. Obviously the carbon dioxide could have been fixed in the simple one-carbon compound urea or formic acid, a fixation far different from that which the term "assimilation of carbon dioxide" suggests to most people.

Assuming that one has completed a simple demonstration of fixation of carbon dioxide in a biological process, what additional criteria must be met then to determine the mechanism of fixation? It is here that the real problems of isotopic work are met, and the problems are by no means simple. Isotopes are a very valuable tool to the investigator, but even isotopes involve many uncertainties. Frankly, the field of isotopic investigation is not fully enough developed to permit a clear understanding of all the criteria that must be met. Therefore, in this short presentation only a few of the possible sources of error will be presented as a starting point for discussion.

In any investigation, whether it involves isotopes or not, the general experimental procedure must be reliable if results are to be valid. In fact, assuming that one is cooperating with a competent physicist, the actual isotopic analysis and separation of isotopes will be the minor problem; the general experimental procedure will offer the real problems. A specific experiment may be cited as an example of a case in which the error resulted from the general procedure and not from any isotopic considerations. In the propionic acid fermentation, propionic acid is formed in which carbon dioxide-carbon is incorporated. To obtain information on the possible mechanism of the reactions concerned in the fixation of carbon dioxide in propionic acid, it was necessary to determine the location of the fixed carbon in the propionic acid molecule. Thus the problem was to select a reliable chemical reaction for the degradation of the molecule. In doing this Carson and his co-workers degraded radioactive propionic acid obtained from the propionic acid fermentation by alkaline permanganate oxidation and obtained oxalic acid and carbon dioxide:

\section{$\mathrm{CH}_{3} \mathrm{CH}_{2} \mathrm{COOH} \rightarrow \mathrm{COOH} \cdot \mathrm{COOH}+\mathrm{CO}_{2}$}

From 70 to 75 per cent of the radioactive carbon was found in the oxalate fraction and 25 per cent in the carbonate. Since the workers believed that the carbonate arose from the carboxyl carbon and the oxalate from the alpha and beta carbons of propionic acid, it ap- 
peared that the carbon dioxide was equally distributed among the three carbons in the chain. In other words, it seeemed that the propionic acid might be synthesized entirely from carbon dioxide, which would be a most remarkable accomplishment for a typically heterotrophic propionic acid organism. It was soon proved, however, that the reaction was not a reliable method of decarboxylating propionic acid. This was done by checking the reaction with synthetic propionic acid, which contained $\mathrm{C}^{13}$ in the carboxyl group. Further, by use of a reliable degradation reaction, all the fixed carbon in the biologically formed acid was shown to be in the carboxyl group. Carson and his co-workers obtained the same results when they rechecked their previous procedures.

With this example we may pass on to consideration of the case, in which it will be assumed the criteria described above have been met, i.e., (1) a reliable determination of $\mathrm{C}^{13}$ has shown there is fixation of carbon dioxide; (2) the experimental procedure has been a good one for demonstrating the desired results; and (3) the compound or compounds containing the fixed carbon have been isolated and degraded by reliable chemical reactions; thus the location of the fixed carbon in the respective compounds is known.

It is true that only by the use of isotopic carbon could such information be obtained, but even then there may be much uncertainty respecting the mechanism of fixation of carbon dioxide. For example, in bacterial glucose fermentations by Staphylococcus, Streptococcus, and Proteus, it has been shown by Slade et al. that the carbon dioxide is fixed in the carboxyl groups of the lactate and succinate; with Aerobacter and Clostridium welchii, there is fixation in the carboxyl group of the acetic acid as well. With pigeon liver on pyruvate, there is fixation in the carboxyl groups of malate, fumarate, succinate, lactate, and alpha-ketoglutarate. Many of these fixations are believed to occur initially by three- and one-carbon addition through the following reaction:

$$
\mathrm{CO}_{2}+\mathrm{CH}_{3} \cdot \mathrm{CO} \cdot \mathrm{COOH}=\mathrm{COOH} \cdot \mathrm{CH}_{2} \cdot \mathrm{CO} \cdot \mathrm{COOH}
$$

Particularly it is believed that this reaction is instrumental in the formation of four-carbon dicarboxylic acids. The mechanism of the fixation in lactate and acetate is still largely unknown. But what criteria can be used in determining the reliability of the suggested fixation by three- and one-carbon addition? At present there is no completely reliable criteria; but this represents our interpretation of the present known experimental facts.

Thus far the synthesis of oxalacetate from pyruvic acid and carbon 
dioxide has not been accomplished, but Krampitz in our laboratories has done the next best thing. With a preparation of Micrococcus, which decarboxylates oxalacetate to pyruvate, he has shown the catalysis of the exchange of $\mathrm{C}^{13}$-carbon dioxide with the carboxyl group of oxalacetate, i.e., incubation of oxalacetate with $\mathrm{C}^{13}$-carbon dioxide and the enzyme gave oxalacetate containing $\mathrm{C}^{13}$ in the carboxyl group. During this exchange the oxalacetate is apparently broken down to a three-carbon compound and again resynthesized, permitting the entrance of $\mathrm{C}^{13}$-carbon dioxide. It is suggested that the three-carbon compound involved in the fixation reaction is not pyruvic acid, as such, but a derivative of this compound that is formed during the decarboxylation of oxalacetate. This is the first direct evidence, i.e., evidence with oxalacetate as such, that has been obtained as proof of the reaction.

Finally, I should like to comment on one further consideration. Assuming that we know the mechanism of the reactions concerned in fixation of carbon dioxide, we are still faced with one very important question, namely, is the fixation reaction an essential step in the dissimilation or is it the result of an exchange reaction, for example? Time does not permit full development of this subject. Furthermore, since the problem has not yet been given much consideration in most investigations, satisfactory criteria have not been devised for determining whether a reaction is essential or not. An example may serve to illustrate the point. In the Krebs cycle, as Dr. Evans pointed out in his lecture, the pyruvate is believed to be oxidized after union with oxalacetate through a cyclic conversion. The oxalacetate, it is believed, arises from the fixation reaction through union of pyruvate and carbon dioxide. The fixation reaction would then be an essential reaction in the oxidation of pyruvate, since it would supply the necessary oxalacetate. It is possible, however, that the oxalacetate cannot be formed by this reaction but is formed by some other reaction of pyruvate. The oxalacetate thus formed might react with carbon dioxide by an exchange reaction as studied by Krampitz. In this case one would arrive at oxalacetate containing heavy carbon just as he would if the oxalacetate was formed by union of pyruvate and carbon dioxide. Isotopic analysis would not serve to differentiate the mechanisms. It is evident that a false importance may be ascribed to carbon dioxide fixation reactions, for fixed carbon dioxide may result from a non-essential exchange reaction. Frankly, I do not believe this to be the case, but I can cite only indirect criteria to support my opinion, such as the fact that carbon dioxide is necessary for growth of bacteria and in the reduction of methylene blue by 
dehydrogenase. If carbon dioxide was concerned only in a non-essential exchange, it would not be required.

\section{R. H. Burris, Columbia University:}

The criteria that must be met in the use of isotopes as tracers have been well covered by Dr. Wood in the preceding discussion, so we may consider certain other problems connected with isotopic tracers. The question is frequently heard, "Which tracers are more suitable for biological problems, radioactive or stable isotopes?" There is no blanket answer, for the suitability of a given tracer depends not only upon the properties and availability of the given element, but also upon the type of experiment in which the tracer is to be employed.

In qualitative studies the radioactive isotopes of reasonably long half-life possess many advantages; they can usually be detected in greater dilution and with greater facility than can the stable isotopes. In addition, they may often be traced directly in vivo without laborious fractionation of the organism. For example, shortly after the ingestion of a radioactive sodium salt radioactivity may be detected in any part of the body if it is brought into proximity to a Geiger counter chamber. Radioactive substances with sufficiently intense radiations will reveal their distribution by forming radiographs on photographic plates. In this manner the pattern of radioactive substances in bones has been demonstrated following the feeding of isotopes.

Radioactive isotopes have been prepared in much greater variety than have the stable isotopes. More than two hundred radioactive isotopes have been produced. Many, of course, are of no biological significance; others have not been prepared in suitably high concentrations for tracer studies; and unfortunately some of the elements of greatest biological interest form isotopes of very short halflife. The following radioactive elements have been used in biological investigations:

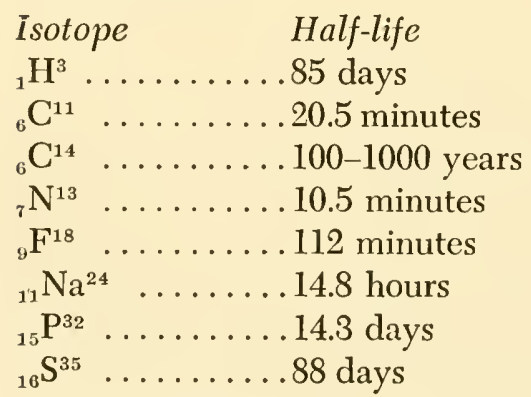

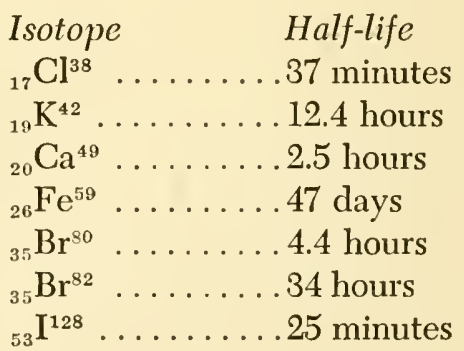


The chief feature in favor of the stable isotopes is, of course, their stability. The extreme speed necessary in handling radioactive isotopes of short half-life, such as $\mathrm{C}^{11}$ and $\mathrm{N}^{13}$, has led to some unfortunate errors, as has been pointed out by Dr. Wood. With the stable isotopes time is not a factor, and compounds can be separated and analyzed at leisure. When isotopes become available for general distribution, the stable forms may be stocked without decomposition.

The objection that radiations from radioactive tracers may injure tissues is scarcely a serious one, since in most cases there is a wide margin between the level of radioactivity necessary for measurement and the level that will be injurious. With the stable tracers the question of radiation does not arise. Only in the case of deuterium, with its marked differences in properties from its analogue, have injury effects been noted when high concentrations of a stable isotope were present.

Although the stable isotopes have not been prepared in as great variety as the radioactive isotopes, the elements of chief interest to the biologist have been concentrated. $\mathrm{H}^{2}$ (deuterium), $\mathrm{C}^{13}, \mathrm{~N}^{15}, \mathrm{O}^{18}$, and $S^{34}$ have been concentrated by Urey. Deuterium is an item of commerce, and $\mathrm{N}^{15}$ and $\mathrm{C}^{13}$ may be on the market soon.

For quantitative experimentation the stable isotopes are far superior to the radioactive isotopes. Rittenberg has found that in the analysis of $\mathrm{N}^{15}$ with the mass spectrometer he can expect a precision of \pm .003 atom per cent $\mathrm{N}^{15}$. To obtain comparable precision in measuring a radioactive substance with a Geiger counter it would be necessary to count in the order of a million impulses. If a Geiger counter capable of handling a thousand impulses a minute were available, and the sample under examination were sufficiently concentrated to give this output of charged particles, sixteen and twothirds hours would be required to register a million counts. It is obvious that radioactive nitrogen with a half-life of 10.5 minutes or radioactive carbon with a half-life of 20.5 minutes could not be measured with precision. Nor is short half-life the only factor that interferes with the quantitative measurement of radioactive isotopes; many of the radioactive isotopes with a long half-life $\left(\mathrm{C}^{14}, \mathrm{~S}^{35}, \mathrm{Fe}^{59}\right.$, etc.) emit such soft radiations that measurement is extremely difficult, and background counts constitute a considerable percentage of the total counts. Radioactive phosphorus is an ideal tracer with a long half-life and an intense radiation, and in this case precise quantitative measurements can be obtained. But with the elements of chief interest to the biologist-carbon, hydrogen, and nitrogen-the 
stable isotopes are much more favored for quantitative studies than the radioactive elements.

The application of isotopes to problems involving respiratory enzymes has not been extensive. The metabolism of lactic acid and the assimilation of carbon dioxide have been traced with stable and radioactive carbon isotopes. The use of radioactive $\mathrm{P}^{32}$ in the study of the role of phosphorus in respiration has attracted a number of investigators. In the near future, undoubtedly, isotopes will be employed in many other studies of this nature.

\section{MECHANISMS FOR THE COMPLETE OXIDATION OF CARBOHYDRATES BY AEROBIC BACTERIA}

\section{H. Werkman, Iowa State College:}

This topic is a broad one that has not been exhaustively investigated because it offers serious technical difficulties. Bacteria seem to possess a distinct and quite troublesome cell wall whose behavior as regards changes in permeability is far from clear. Realizing the danger of drawing conclusions from the use of unnatural systems, we have attempted, by correlating the results obtained with juices and whole cells, to circumvent the pitfall. But to do so would apparently require an uncanny ability, since neither the work with juices nor the cell suspension could be accepted as portraying processes that occur in the living, reproducing organism. In the face of this difficulty of preparing juices or cell suspensions that behave naturally, the task of elucidating the natural processes in bacterial respiration is laden with danger. Our present purpose is to encourage a free discussion of the possibilities in bacterial respiration and to outline a working hypothesis.

There is abundant evidence that phosphorus plays an important, if not essential, role in bacterial respiration. Early workers, including Virtanen, have shown the ability of bacteria to form phosphorylated esters. Wiggert in our laboratory showed an uptake of phosphorus by living bacteria. That the principles of the Embden-Meyerhof scheme of glucolysis operate in bacterial metabolism was first given substantial support in 1936, when the characteristic intermediate of that scheme, phosphoglyceric acid, was isolated from representative types of bacteria, both aerobic as well as anaerobic (Werkman et al., Stone and Werkman). Utter in our laboratory has shown the occurrence of the aldolase reaction by means of bacterial juices. In this reaction hexosediphosphate is converted into phosphoglyceraldehyde and dihydroxyacetone phosphate. Furthermore, he has estab- 
lished the dissimilation of phosphoglyceric to pyruvic acid through phosphopyruvic.

Pyruvic acid has been shown to be a general intermediate in the dissimilation of glucose by bacteria. In the case of anoxybiontic metabolism the pyruvic acid is converted into a variety of products; in the case of oxybiontic (aerobic) metabolism, however, it appears that pyruvic acid initiates the changes of terminal respiration. These are the changes of the whole aerobic dissimilation, and provide for the oxidation of pyruvic acid to carbon dioxide and water. With respect to bacteria there is evidence that the final processes of respiration involve the cytochrome-cytochrome oxidase system. Both cytochrome and its oxidase have been shown to be present in many aerobic bacteria, and in no case known has cytochrome failed in an aerobic species. There is no doubt that bacteria possess a cytochrome mechanism, although many questions respecting the details of its operation remain to be answered. We have found, for instance, an acetone-resistant cytochrome oxidase not reported in animal tissues, and bacterial cytochrome may have a lower potential than that in animal tissue.

Between the terminal stage of glycolysis and the initial stage of the cytochrome mechanism there is a portion in the spectrum of respiration that has received only passing investigation so far as bacteria are concerned. This may be referred to as the four-carbon dicarboxylic acid portion. The Szent-Györgyi and Krebs schools have pioneered in this work as it relates to animal tissue, and our lead is taken from their work.

It is this four-carbon acid portion which we wish to discuss first. Mr. Krampitz in our laboratory has been wrestling with these secrets of nature. A few experiments and results will be cited here for purposes of discussion. It is hardly necessary to mention that our remarks are preliminary.

Shortly after Szent-Györgyi formulated his theory of the role of the four-carbon acid in cellular physiology, an attempt was made to apply it to bacteria. Before any catalytic effect of the fourcarbon acids can be shown, the preparation of enzymes must be made deficient in four-carbon acids by washing bacteria free from these acids. Using Micrococcus lysodeikticus to make our preparations, we did obtain stimulation with fumaric acid varying from 35 to 120 per cent as measured by oxygen uptake. Such stimulation was not, however, obtained consistently, and therefore we attempted to increase the cell permeability by acetone treatment, hoping to re- 
move the four-carbon acids more effectively. But this preparation did not function in all respects like normal cells, failing to oxidize glucose and succinate among others, although it did oxidize the fourcarbon acids (fumaric and malic) and lactate and pyruvate to acetic acid. Since succinate was not oxidized, we believed that the cytochrome system might have been injured, inasmuch as the hydrogen, according to Szent-Györgyi, is transmitted through succinate to the cytochrome system and thence to oxygen. When methylene blue or cresyl blue was added to the system as carriers of hydrogen to replace the cytochrome system, no oxidation of succinate occurred. Injury to flavoprotein action appeared ruled out because of the rapid turnover of the substrates that were oxidized, i.e., fumarate, malate, etc. Since acetone is known to destroy cytochrome oxidase, it was difficult to understand the rapid attack on fumarate, malate, lactate, and pyruvate. It was shown spectroscopically that the preparation contained a cytochrome oxidase resistant to the acetone treatment. These results were enough to indicate that investigations of bacterial respiration were to prove interesting as well as a bit troublesome.

One of our principal objectives has been to integrate the heterotrophic assimilation of carbon dioxide and respiration. We have therefore run a number of experiments to gain a better insight into the mechanism of bacterial respiration. The evidence so far accumulated seems to indicate that the Szent-Györgyi cycle does not function in bacterial respiration. Glucose, for instance, is not attacked by Micrococcus lysodeikticus anaerobically by cell suspensions or the acetone preparation. If the Szent-Györgyi system were operating, malic acid present would be oxidized anaerobically, since fumarase would provide fumaric acid as a hydrogen acceptor for the system. This does not occur.

Thus far the evidence favors the occurrence in principle of the Krebs citric acid cycle in bacteria. As the Krebs cycle is presented, every alpha-keto acid with the single exception of pyruvic acid is oxidatively decarboxylated. This point should be further investigated to determine whether pyruvic acid is not also oxidatively decarboxylated, not necessarily to acetic acid, but to some two-carbon compound which is able to condense with oxalacetic acid to initiate the citric acid cycle. Acetic acid is commonly found as an end-product when juices or perhaps injured cells are employed. Maintenance of an adequate supply of oxalacetic acid is a requirement of the Krebs scheme, and this is assured by regeneration in the cycle and by the utilization of carbon dioxide through the Wood and Werk- 
man reaction. We have found some evidence for the formation of citric acid in the presence of acetic acid by Micrococcus lysodeikticus.

On the other hand, many bacteria do not appear to metabolize citric acid; this is difficult to reconcile with the Krebs cycle as proposed, although it is probable that it is not citric acid as such which is the intermediate.

At present we are at work on $\mathrm{C}^{13}$ acetic acid as a tracer. Certain experimental evidence with the four-carbon acids has at times suggested that we are dealing with phosphorylated compounds, probably of a very labile nature. Thus an acetone preparation is made magnesium- and cocarboxylase-deficient by alkaline phosphate washing of the cells as determined by testing on pyruvic acid. The deficient preparation does not decarboxylate oxalacetic acid; however, the addition of magnesium ions completely restores the activity. The reaction yields carbon dioxide and pyruvic acid. When malic acid replaces oxalacetic acid, the deficient preparation under the same conditions does not oxidize malic acid to carbon dioxide and pyruvic acid, but only to oxalacetate, which accumulates and does not inhibit the bacterial malic dehydrogenase as it does tissue dehydrogenase. The complete preparation (deficient plus magnesium ions or the unwashed acetone preparation) oxidizes malate to carbon dioxide and pyruvic acid with traces of oxalacetic acid. Thus with laboratory oxalacetic acid the reaction goes to carbon dioxide and pyruvate, whereas with "physiological" oxalacetic acid (from malate) the reaction appears to maintain an equilibrium.

Is the "physiological" oxalacetic acid different from that prepared in the laboratory, possibly a phosphorylated compound? We investigated the problem and at one time thought that it was. Definite stimulation by phosphate has been demonstrated for fumaric or malic acid. As yet we have not shown a phosphate uptake, or isolated an organic phosphate; however, we may be dealing with a labile carbonyl phosphate in the sense of Lipmann. It is suggested that the carbonyl group is bound in the physiological oxalacetate formed from malic acid, since traces of oxalacetate are known to inhibit malic oxidation in tissue. If malate is oxidized in an atmosphere of $\mathrm{C}^{13} \mathrm{O}_{2}$ and $\mathrm{O}_{2}$, the oxalacetic acid contains $\mathrm{C}^{13}$ in the carboxyl group adjacent to the methylene group. No chemical exchange takes place. The enzymatic decarboxylation of oxalacetic acid in the presence of heavy carbon dioxide also yields heavy carbon oxalacetic acid. This is a form of carbon dioxide utilization. 


\section{E. S. Guzman Barron, University of Chicago:}

Until a few years ago scme investigators in the field of oxidationreductions tended to devo'te their efforts exclusively to animal, plant, or bacterial oxidations. Many papers published recently showing the variety of oxidation mechanisms, even in the oxidations involving simply an electron transfer, have demonstrated the necessity of integrating the facts obtained with these different kinds of living beings. For such comparative studies, work with bacteria has been fruitful not only because it is possible to obtain suspensions or extracts with which quantitative studies can be performed but also because in a single species, say hemolytic streptococci, a variety of oxidation mechanisms may be found in different strains.

If we take the component of oxidation enzyme systems closest to molecular oxygen, the iron porphyrins, we may divide bacteria into two groups: cytochrome-containing bacteria (including most of the so-called aerobic bacteria) and cytochrome-lacking bacteria (the socalled anaerobic bacteria). Species of the two groups may produce idlentical oxidations. They may oxidize, for example, lactate or glycerol. The rxidation of lactate and glycerol by cytochrome-containing bacteria (Staphylococcus) is completely inhibited by cyanide, whereas the same oxidations by cytochrome-lacking bacteria are cyanide-insensitive. Obviously iron porphyrins take part in the oxidation of lactate and glycerol by cytochrome-containing bacteria, whereas in cytochrome-lacking bacteria the oxidation proceeds through different channels (flavin nucleotides).

In the field of phosphorylative oxidations the laboratory of Werkman has demonstrated that there exist in bacteria the different phosphorylations observed in the breakdown of carbohydrate by muscle or yeast extracts. In our laboratory it has been found that the oxidation of glycerol by hemolytic streptococci does not take place in the absence of phosphates. This does not mean that phosphorylation is essential for glucose oxidation; it is known that the breakdown of carbohydrate by molds proceeds without phosphorylation.

It is assumed that in animal tissues carbohydrate metabolism starts with the fermentation process ending in lactate, whereas in yeast it ends in the formation of alcohol. In bacteria the fermentation process may end in the formation of either lactate or of alcohol or in the formation of both end products, as Friedemann has shown. Fermentation may be absent altogether, as in glucose-non-fermenting bac- 
teria. These bacteria either oxidize glucose directly without previous phosphorylation (Pseudomonas aeruginosa) or oxidize directly phosphorylated hexose (hexose monophosphate and diphosphate).

The same variety of mechanisms is found in the oxidation of pyruvate $\left(\mathrm{CH}_{3} \mathrm{COCOOH}+\frac{1 / 2}{12} \mathrm{O}_{2}=\mathrm{CH}_{3} \mathrm{COOH}+\mathrm{CO}_{2}\right)$ : it requires an iron porphyrin catalyst in gonococci; it proceeds without iron porphyrin in Bacterium Delbriickii, as Lipmann has shown. d-Amino acid oxidase, isolated by Warburg and Christian, is an alloxazin dinucleotide protein, the oxidation of alanine to pyruvate being cyanide-insensitive; this oxidation when performed by cytochromecontaining bacteria requires iron porphyrin as a component of the enzyme system because the oxidation is completely inhibited by cyanide.

These examples are presented as proof of the existence of multiple mechanisms of oxidation. A comprehensive study of biologic oxidation-reduction demands, therefore, a continuous and simultaneous attention to the oxidation mechanisms throughout living cells.

P. W. Wrison, University of Wisconsin:

A discussion of oxidations by aerobic bacteria should certainly include reference to the fact that one of the most actively respiring tissues known belongs to this group of organisms. I refer, of course, to the extremely high rate of respiration possessed by certain cultures of Azotobacter, the free-living nitrogen-fixing bacteria. One of our distinguished guests and participants, Dr. Otto Meyerhof, first called attention to this several years ago when he reported $\mathrm{Q}_{\mathrm{O}_{2}}$ values of 500 to 8600 for Azotobacter chroococcum on glucose at $28^{\circ} \mathrm{C}$. $(1,2)$. The extremely high values were obtained with very young cultures so diluted that the total dry weight involved was less than 10 micrograms. Its estimation may have been subject to some error, but it is probable that young cultures of this organism have a $\mathrm{Q}_{\mathrm{O}_{2}}$ value of at least 5000 .

Since Meyerhof and his collaborators made these experiments, important advances have been made toward developing cultural conditions that are optimum for growth and nitrogen fixation by Azotobacter. Rates of fixation are consistently obtained in experiments today which are several times greater than those reported several years ago. For example, Azotobacter vinelandii can fix as much as $20-30 \mathrm{mg}$. of nitrogen in $24-36$ hours instead of $4-5 \mathrm{mg}$. in one or two weeks which was the characteristic result of most of the 
earlier studies (3). It is of interest to examine such cultures for respiratory activity. My associate, Dr. R. H. Burris, has determined the $\mathrm{Q}_{\mathrm{O}_{2}}$ values of a 12-hour culture of Azotobacter agilis on a number of substrates; his findings were as follows: endogenous, 28; glucose, 29; lactate, 129; arabinose, 29; acetate, 1109; ethyl alcohol, 1240. When a 48-hour culture was used, these values were greatly reduced.

More recently Mr. Joe Wilson in our laboratory has made similar observations with Azotobacter vinelandii. In most of these studies a 24-hour culture was diluted, and the oxygen uptake measured immediately for a period of 60 minutes. The substrate was sucrose, the temperature $30^{\circ} \mathrm{C}$., the $\mathrm{pH}$ 7.0. Air was used as the gas phàse so that opportunity for growth existed, but no detectable fixation of nitrogen occurred during this short experimental period. To avoid the error attached to estimation of dry weight, he calculated the rate of respiration on the basis of cell nitrogen (4). Since these cells of Azotobacter contain about 10 per cent nitrogen, such $\mathrm{Q}_{\mathrm{O}_{2}}(\mathrm{~N})$ values are on the average about 10 times as great as the $\mathrm{Q}_{\mathrm{o}_{2}}$ based on dry weight. Under these conditions the values of the $\mathrm{Q}_{\mathrm{o}_{2}}(\mathrm{~N})$ ranged from 25,000 to 30,000 .

These data emphasize the extremely high rate of respiration of different species of Azotobacter and suggest that this organism may well provide an excellent source for the preparation and isolation of different enzyme systems concerned with the transfer of hydrogen from substrate to molecular oxygen. With this in mind we are investigating methods for growing Azotobacter on a scale considerably greater than any previously attempted. In a pilot plant designed for yeast production we have succeeded in producing several pounds of moist azotobacter cells during a growth period of 24 to 30 hours.

\section{REFERENCES}

1. Meyerhof, O., and Burk, D., Z. physik. Chem., 139A, 117 (1928).

2. Meyerhof, O., and Schulz, W., Biochem. Z., 250, 35 (1930).

3. Wilson, J. B., and Wilson, P. W., Jour. Bact., 42, 141 (1941).

4. Burrus, R. H., and Wilson, P. W., Proc. Soc. Exp. Biol. Med., 45, 721 (1940).

\section{REACTIONS IN CELL-FREE ENZYME SYSTEMS COMPARED WITH THOSE IN THE INTACT CELL}

\section{F. F. Nord, Fordham University:}

In interpreting the results of investigations of bacterial metabolism a few principles should be mentioned which, in addition to the very recent use of tracers, appear to have been applied in approaching 
the problems with which we are confronted in extracts and in living cells:

1. In extracts a disturbance of the ratio of the various components and an effect upon the total enzyme system occurs automatically, which may cause an accumulation and even a stabilization of transient products.

2. By selective poisoning of parts of the enzyme system numerous facts have been established.

3. In living cell processes, because of the introduction of reagents not akin to the whole system, a supposed or possible intermediary product is removed and thereby excluded from the reaction sequence. For example:

1. Amino acid oxidase is capable of deaminating $l$ - or $d$-amino acids in tissue slices. In the case of injured or denatured tissues, however, natural amino acids are no longer deaminated.

2. The extent to which the carrier enzymes are dispersed may change under the influence of various factors. Moreover, in the cells disperse particles of the various protoplasmic substances actually possess widely differing $\mathrm{pH}$ values.

3. Even Dr. and Mrs. Cori declare, in accordance with the aforementioned fact, that the conditions for glycogen synthesis are much more favorable in the intact cell than in tissue extracts, where, they state, they have obtained starch. ${ }^{*}$

4. Experiments show that in the case of Corynebacterium diphtheriae, strains gravis and mitis, conditions are comparable.

5. There is no stoichiometrical relationship between the carbon dioxide cvolved and the actual decrease in inorganic phosphorus (in the living cell).

6. When carbon dioxide evolution was compared with energy liberated as heat by living yeast cells and by Lebedew extract, it was noted that the heat of reaction in the course of fermentation changed continually, indicating that fermentation with living cells does not proceed according to a fixed scheme.

The thermochemical course of fermentation with juices shows, in contrast, that at least two different conversions occur: (1) fermentation of free sugar in the presence of free phosphate (inhibited by phloridzin) and (2) the subsequent fermentation of the residual substrate in the absence of free phosphate (not inhibited by phloridzin).

* Compare G. J. Goepfert, Brewers Digest, 16, No. 6 (1941). 


\section{P. W. WILson, University of Wisconsin:}

It is only recently that most investigators of bacterial metabolism have had the opportunity to choose between cell extracts and intact cells. Because of their small size, bacteria as a group have resisted attempts to destroy their cellular integrity. In the past, if studies on bacterial enzyme systems were to be made, the investigator was in the position of Kipling's thief who took the hot stove because there was nothing else that season-he had to use intact cells. Although this limitation has undoubtedly complicated the investigations and the interpretation of the results, it has not been insurmountable. By use of the so-called "resting cell" technique, great strides have been made toward an understanding of the biochemistry of bacteria. With respect to individual enzyme systems the greatest success has attended studies in which isolation of the reaction is made possible through choice of substrate rather than enzyme system, for example, hydrogenase and hydrogenlyase. When less specific substrates are employed, great care must be exercised that the interpretation is not oversimplified by applying information gained in what appears to be an analogous study made with the more purified enzyme preparations.

With certain bacterial enzyme systems the investigator is denied even the use of non-proliferating cells. For example, studies on nitrogen fixation by Azotobacter must usually be made with growing cultures: on the symbiotic system with a very complex association of bacteria and host plant. Despite these technical handicaps, the development of certain methods $(1,2)$ during the last decade has enabled investigators to secure what Burk has recently described as "the most intimate information that we possess on the mechanism of fixation, and in particular on the nature of the first crucial step involved" (3).

In recent years special techniques have been developed which allow cell-free extracts containing a variety of enzymes to be prepared from bacterial species. While it is gratifying that this first step toward isolation of individual enzymes has been made, the immediate practical value of the achievement has not been great. In most cases the net result of the separation has been the verification of a portion of the knowledge previously obtained with the resting cells. Recently we have prepared a cell-free Azotobacter "juice" which contains among other enzymes very powerful preparations of hydrogenase and oxalacetic decarboxylase. Our satisfaction over this ac- 
complishment is somewhat lessened by our recognition that we can do little with the extract that we have not already done with intact resting and acetone-treated cells. Nevertheless, we continue studies with it in the hope that a clue to the mechanism of nitrogen fixation may be furnished by the extract that has been successfully hidden in the intact organism. Meanwhile, however, the physical-chemical studies on the intact growing bacteria will not be neglected, since they have already proved their value.

\section{REFERENCES}

1. Burk, D., Ergebnisse d. Enzymforschung, 3, 23 (1934).

2. Wrison, P. W., The Biochemistry of Symbiotic Nitrogen Fixation (University of Wisconsin Press, 1940).

3. Burk, D., and Burrus, R. H., Ann. Rev. Biochem., 10, 587 (1941). 


\section{Discussion on Animal Tissue Respiration}

\section{A. ELVEHJEM, University of Wisconsin, Chairman}

\section{FACTORS AFFECTING THE PREPARATION OF TISSUE FOR METABOLIC STUDIES \\ EPHRAIM SHORR \\ Cornell Medical College, New York}

My comments will be restricted to certain difficulties encountered in the preparation of tissue for in vitro studies of metabolism. They touch on the various methods of preparing tissue, such as the slice and mince method and on the influence of certain chemical changes that are inevitable during the handling of the tissue prior to the experimental run.

The Slice Method.-Most workers use the limiting formula of Warburg without testing the permissible thickness of the specific tissue with which they are working. The general tendency is to get a slice as thin as possible. Histological studies, as well as comparative studies of the rate of respiration, show that this is not altogether wise. The superficial layers can be shown to undergo degeneration to variable depth. The thinner the slice the larger is the proportion of damaged tissue. This is particularly important for tissues such as cardiac muscle, where a whole large cell unit at the surface must inevitably undergo degeneration. Thicker slices can be shown to have a higher rate of respiration than very thin ones. The maximum thickness which is permissible is therefore better. Not infrequently thicknesses which exceed the formula behave very well. This points to the possibility that there may be mechanisms for maintaining oxygen pressure other than the gradient set up by the tension in the solution-perhaps the iron-carrying compounds of the tissue, which serve as a storehouse. The slice method is of course best adapted to parenchymatous organs, least well to muscle. Unfortunately cardiac muscle does not lend itself to dissection as does skeletal. However, the individual muscle cells are much shorter than skeletal muscle cells, hence the degeneration occurring at the surface is not extensive enough to do much harm. As regards the brain, there seems to be little evidence that the more convenient method of chopping the tissue with a razor is less good than slicing. 
The Muscle Strip Technique.-In most experiments reported in the literature where skeletal muscle has been employed, it has either been minced or chopped with scissors if larger animals have been used, or the diaphragm of a small animal, such as the rat, has been employed intact. The chopping or mincing is extremely destructive, sets up abnormal chemical processes in the presence of oxygen, such as aerobic glycolysis, and permits brief survival. The rat diaphragm, while excellent for many purposes, is small, permits of few concomitant chemical measurements, and has only a limited usefulness because of the fact that many metabolic conditions, such as diabetes, cannot be brought about in this animal. The dog is a much better experimental animal in these respects. The neck muscles of the dog are ideal for obtaining, by careful dissection, long muscle strips for in vitro studies. Individual fibers can be teased out intact. These maintain their histological integrity for long periods. They respond to electrical stimulation for hours unchanged, and give reproducible results as far as work and heat production. Furthermore, enough material can be obtained to allow for extensive chemical balances. It is, I am sure, the method to be used when this type of tissue is employed with in vitro studies.

Minced Tissue.-Here the choice lies between using a Latapie mincer or a homogenizing apparatus described by Potter. Histological examination of the tissue obtained by these two methods shows that with the homogenizer the tissue is completely and uniformly disintegrated, whereas with the Latapie the destruction is not complete and the tissue is a mixture of disintegrated and intact cells. The amount of destruction differs with the organ, and these differences are paralleled by differences in the rate of respiration. For example, skeletal muscle because of its long fiber is completely destroyed by both methods and the rate of respiration is the same. With the parenchymatous organs, such as the liver and kidney, a definite difference in respiration results from the two methods, the respiration of the homogenized tissue being lower than that of the Latapie. The same is true of cardiac muscle. The higher respiration may be due to the presence of the intact cells in the tissue put through the Latapie. Obviously tissues minced by these two methods are not comparable with respect to respiration, and other differences may exist. This discrepancy should be borne in mind whenever the two methods yield different results. It would certainly seem desirable to decide on one or the other. My preference would be homog- 
enization because of its uniformity. With this latter method, overheating of the solution during the stirring should be rigorously avoided. With minced tissue from the Latapie, two methods of obtaining aliquots are commonly employed. The minced tissue may be weighed out as such or stirred up in a cool solution and pipetted out. My preference is for the latter method, which is a simple one if a wide-mouth pipette is used. It is also much more rapid and yields somewhat more uniform respiration in duplicates and triplicates. With the other method it seems difficult to keep all the tissue under the same conditions of cooling, a desideratum when things are happening as rapidly as they do in such a tissue. Furthermore, I have no confidence in single experiments and so would urge that triplicate determinations be carried out.

Complicating Factors Arising between Removal of the Tissue and the Experimental Run.-During this inevitable unphysiological period in which the tissue is anaerobic, many breakdown processes occur. These lead to the accumulation of a number of metabolites which can influence the results, especially during the early part of the experiment. Whatever the speed of preparation, these changes cannot be avoided. Those I have had to deal with have been the accumulation of lactic acid, and striking changes in the hexosemonophosphates, adenosinetriphosphate, and phosphocreatine. The accumulation of lactic acid influences markedly the initial rate of respiration, and may suppress the effect of added lactate. In short experiments it may lead to erroneous conclusions respecting the rates of respiration of individual tissues. The literature contains not a few instances of such misconceptions. It can be dealt with either by reducing the lactate content prior to the experiment, by aerating the tissue in a Ringer solution long enough to bring the content to the normal value, or by allowing the excess lactic acid to be dealt with in the micro respiration vessels for an hour or so before the experimental run. The former appears to be much less time-consuming and just as effective. Oxygen is bubbled vigorously through the solution containing the tissue. This is kept at room temperature. As regards changes in the organic phosphate compounds during this interval of preparation, it is very important to restore the normal relationships existing prior to the experiment; otherwise the recovery process may be taking place during part of the experimental period, confusing the results of concomitant chemical balance experiments. This is particularly important for muscle tissue, and it is necessary to determine, for each tissue, how long an equilibration is required to 
permit complete recovery. Undoubtedly other accumulations and disturbances occur which we have not detected. This is worthy of investigation for any specific system under study.

The Escape Phenomenon.-Where in vitro experiments are prolonged, another type of phenomenon must be borne in mind. We have shown that diabetic tissue on prolonged survival in vitro gradually regains its ability to oxidize carbohydrate. A complete restoration takes place in four hours at $41^{\circ} \mathrm{C}$., in ten hours at $37.5^{\circ} \mathrm{C}$. With cardiac muscle a definite elevation in the respiratory quotient of normal tissue is found as early as the second hour. This change has been attributed to the release of the tissue from certain influences carried over from the intact animal. The change can be checked by the use of other than inorganic phosphate buffers. Among these is beta-glycerophosphate. In prolonged experiments with any tissue this phenomenon should be borne in mind, since, it may be associated, as in this instance, with an entirely different type of metabolism toward the end of the experiment than at the start.

Miscellaneous.-In obtaining respiratory quotients it has been our experience that for vessels of any given size more reliable results are obtained when the respiratory exchange is large. When the total oxygen consumption and carbon dioxide production is small, the results are likely to be unreliable, and generally the respiratory quotient is erroneously high. In vessels of 20 to 24 cc. capacity, if the oxygen consumption in the period of observation is less than $150 \mathrm{cmm}$., we are likely to obtain incorrect respiratory quotients. It is recommended that for each size and type of vessel used, studies be made to determine the amount of oxygen consumption that yields a reliable respiratory quotient.

\title{
COMPARISON OF SLICES AND HOMOGENIZED SUSPEN- SIONS OF BRAIN TISSUE
}

\author{
K. A. C. ELLIOTT \\ Institute of the Pennsylvania Hospital, Philadelphia
}

Warburg introduced the technique of using slices of tissue for metabolic studies, and the method has been used by many other workers. Slices of many tissues can be prepared without disrupting the majority of the cells; gases and substrates can diffuse in and out of thin slices rapidly enough not to limit the rates of metabolic processes. With various mashed and ground preparations, respiration has been found to occur less rapidly, and it is commonly believed that a closer approach to physiological conditions is obtained with 
slices than with tissue breis. While slices have been used in attempts to determine what a tissue actually does, breis have been useful in discovering essential substances and the interrelation of reactions. The disintegration of tissue permits various essential materials to be more readily diluted by the suspending medium or destroyed by enzymes, so that their concentration falls below the optimal and their addition produces striking effects often not found with slices.

The preparation of slices from some tissues, especially brain, is slow and delicate work, sampling is inaccurate, and one cannot be sure that the individual slices do not vary in activity. Dr. Libet and I (1) have recently studied the respiration of brain suspensions and we find that two types of suspension can be obtained. One type behaves as disintegrated tissue; the other type, when prepared under proper conditions, behaves very similarly to slices, shows a comparable respiration rate, and is a suitable and very convenient preparation for the study of brain metabolism.

The first type of suspension is obtained when brain tissue is homogenized by the apparatus of Potter and Elvehjem (2) in hypotonic medium, dilute phosphate buffer solution. Such suspensions respire at a low rate which may be increased up to 65 per cent by adding salt or sugar after homogenization. With such preparations it is therefore necessary that the osmotic pressure be equal in the control and experimental flasks when the effect of added substances is tested. These hypotonic suspensions have largely lost the power to utilize glucose. They show considerable effects when tissue extract or substances like fumarate are added. The rate of respiration per unit weight of tissue increases with increasing tissue concentration.

The second type of suspension is obtained when the medium in which the brain is homogenized contains sufficient salt, sucrose, or glucose to make the osmotic pressure equal to that of serum. The respiration rate of such suspensions is up to 400 per cent greater than the rate of tissue homogenized in hypotonic medium. (Isotonic urea behaves like hypotonic solution.) Suspensions of whole brain (containing a large amount of white matter, which respires only slowly), prepared in $0.13 \mathrm{M}$ sodium chloride- $0.017 \mathrm{M}$ phosphate buffer solution, respire on the average at 71 per cent of the rate of an equal tissue weight of slices of pure gray matter in the same medium. Such suspensions are much less affected by the addition of tissue extract, fumarate, etc., than are hypotonic suspensions, and their respiration rate per unit weight is practically independent of the tissue concentration. Isotonic suspensions respire with the same res- 
piratory quotient as slices, and are similarly affected by additions of various substrates and by variations in the ionic content of the medium. An exception to this rule is that the respiration of slices, unlike that of suspensions, is less well maintained in isotonic sucrose than in isotonic saline solution. When isotonic suspensions are used, air may be used in reaction flasks instead of oxygen. This is an advantage, since it has been found that, while the respiration rates in the presence of air and in the presence of oxygen are identical for 90 minutes, the rate in the presence of oxygen falls off much more rapidly thereafter. A similar slowly appearing toxic effect of oxygen can also be demonstrated with slices, but with slices the presence of oxygen is necessary, since otherwise the initial respiration rate is limited by inadequate diffusion of oxygen into the tissue.

After homogenizing fresh brain in isotonic medium, the rate of respiration falls off very rapidly at first, thereafter less rapidly. Slices and isotonic suspensions prepared from slices do not show the initial very rapid decrease in rate. This suggests that a specially labile part of the respiratory activity can be observed with suspensions of fresh tissue, but is lost during the slow process of preparing for experiments on slices.

Salts induce specific as well as osmotic effects. Isotonic solutions of sodium chloride, nitrate, sulfate, and especially phosphate increase the initial respiration rate appreciably when added to suspensions in isotonic sucrose. Bicarbonate in physiological concentration has no special effect. Calcium and magnesium ions have inhibitory effects on the respiration but cause better maintenance of rate. Potassium in high concentrations is also inhibitory. The initial inhibitory effect of magnesium in the concentration found in serum is fairly small, and this ion is known to take part in reactions of carbohydrate metabolism. For studies of brain respiration, tissue homogenized in isotonic sodium chloride medium containing magnesium is therefore recommended.

However, respiratory activities measured on suspensions or slices in any given medium cannot be considered to represent the true physiological activity of brain tissue in vivo until more is known of the effective concentrations of ions in the cells' immediate environment in vivo and until more is known of the differences that may exist between brain in its normal physiologically active condition and the tissue which has been subjected to the abnormal injuries and stimuli of in vitro work.

The marked difference between tissue homogenized in isotonic 
and in hypotonic media occurs to some extent with certain tissues other than brain, but not with all tissues. Preliminary studies would have to be made on each tissue to which the method of isotonic suspensions is to be applied before it could be assumed that the behavior of suspensions would be comparable to that of slices of the particular tissue.

\section{REFERENCES}

1. Elliott, K. A. C., and Libet, B., in press.

2. Potter, V. R., and Elvehjem, C. A., J. Biol. Chem. 114, 495 (1936).

\section{THE HOMOGENIZED TISSUE TECHNIQUE, THE DILUTION EFFECT AND ION EFFECTS \\ VAN R. POTTER \\ McArdle Memorial Laboratory, University of Wisconsin}

We have developed the homogenized tissue technique for the study of isolated phases of metabolic activity. Since the enzymes that catalyze biological oxidations are in many cases extremely labile, we have used the device of homogenization to effect a physical isolation of a particular enzyme system by dilution. We believe that by so doing we can retain the original activity of the tissue and thus develop assay methods for specific enzymes, where separation by chemical treatment, such as fractional precipitation, could not result in 100 per cent yields and hence would be useless for assay purposes. Since certain components of various enzyme systems are readily soluble and are capable of diffusing away from each other or from solid phases, such as cytochrome oxidase, it is necessary to fortify the homogenate with these diffusible components. Whether it is necessary to add these accessory factors is determined by measuring the enzyme activity at various dilutions. If the measured effect is proportional to the amount of enzyme used, fortification is unnecessary. With regard to choice of buffer, it should be pointed out that since we are now dealing in terms of intracellular components, buffers based on extracellular fluids (such as serum) may not necessarily be optimum.

A system which may illustrate these points is the succinoxidase system. It is inhibited by chloride ions, hence these are omitted from the buffer medium. It requires at least three soluble components for maximal activity, namely, cytochrome $c$, calcium ions, and aluminum ions. The dehydrogenase and the cytochrome oxidase appear to be associated with solid particles of protoplasm. When the dissociable 
factors are supplied, the activity of the system is just as great as that of intact tissue.

More complex are the coenzyme systems in which two additional factors are soluble, namely, the dehydrogenase and the coenzyme. However, by means of fortification with the appropriate coenzyme and inhibition of the coenzyme nucleotidase it should be quite feasible to study these complicated systems in tissue homogenates.

\section{THE STIMULATORY EFFECT OF CALCIUM UPON THE SUCCINOXIDASE ACTIVITY OF RAT TISSUES}

\section{A. E. AXELROD}

University of Wisconsin

Variations in the ionic composition of the medium in which the surviving tissue respires are known to exert profound effects upon the extent of the respiration. Our attention was drawn to these ionic effects by the observation that calcium (as calcium chloride) stimulates markedly the succinoxidase activity of minced rat liver. This phenomenon was investigated further in the succinoxidase system of tissue homogenates prepared according to Potter and Elvehjem. The following results were obtained. In the absence of added cytochrome $c$ the succinoxidase activity of minced liver was increased 43 to 80 per cent by the addition of 20 micrograms of calcium. With homogenized liver ( $40 \mathrm{mg}$. per flask) the addition of 20 micrograms of calcium resulted in increases of 93 and 48 per cent in the absence and presence, respectively, of added cytochrome $c\left(3 \times 10^{-8}\right.$ mole per flask). The succinoxidase activity of homogenized kidney cortex (20 mg. per flask) was stimulated 40 per cent in the presence of added calcium. Added cytochrome $c$ did not affect the magnitude of the stimulatory effect of calcium in this tissue. The most pronounced effect of calcium was observed in the case of homogenized heart tissue ( $20 \mathrm{mg}$. per flask), in which the addition of 20 micrograms of calcium in the presence of $3 \times 10^{-8}$ mole of cytochrome $c$ caused an increase of 200 per cent in the succinoxidase activity. Under our experimental conditions the addition of 20 micrograms of calcium always yielded the maximum stimulatory effect. In many cases the addition of smaller amounts of calcium (as little as 1 or 2 micrograms) resulted in a marked acceleration of succinoxidase activity. In only a few isolated cases was a calcium effect observed in brain and skeletal muscle.

The application of the homogenized tissue technique to the study 
of isolated enzyme systems has been discussed by Dr. Potter, who pointed aut that all precautions must be taken to assure complete restoration of the diffusable factors necessary for the maximal activity of the enzyme system under consideration. The stimulatory effect of calcium upon the succinoxidase system in tissue homogenates necessitates the addition of calcium to such a system if the maximal activity is to be attained. A dissociable complex involving calcium is indicated. The addition of aluminum ions has been shown by Dr. Potter to overcome an effect resulting from further dilution of the tissue. It thus becomes apparent that when either minced liver preparations or tissue homogenates are employed as the source of the succinoxidase system, it is necessary to add calcium and, under certain conditions, aluminum in order to eliminate these ions as possible limiting factors which may affect the validity of the succinoxidase assay.

\section{TISSUE METABOLISM IN VITRO AND IN VIVO}

FREDERICK BERNHEIM School of Medicine, Duke University

The work of Battelli and Stern in the early part of the century may be said to have begun the work on tissue metabolism in vitro which has culminated in the isolation of a number of dehydrogenases. Some of the enzymes which they showed to be present in tissue suspensions have since been shown to be of importance in the economy of the animal. In particular, all subsequent work on the physiology and pharmacology of alcohol has shown that its fate in the body can be accounted for by the activity of the alcohol oxidase of liver which they discovered. Some time later Warburg studied the effect of cyanide on isolated tissues, and from this work came the discovery of cytochrome oxidase and the cytochromes. Again, work on the pharmacology of cyanide has shown that its action on the animal can be explained on the basis of its inhibition of the cytochrome oxidase. In these two early groups of experiments the correlation between in vitro and in vivo results is good. With more recent work similar correlations have either not been made or have not been satisfactory.

The justification for working with broken cell suspensions or cell extracts is that it is only by this means that intracellular enzymes can be studied. The results thus obtained can indicate only that the cell or cell catalyst has certain potentialities under the given set of conditions. Under no circumstances are such results in themselves evi- 
dence for the normal activity of the cell. Attempts to make the conditions more "normal" by adding physiological salt solutions to tissue suspensions is essentially paradoxical, for it is known that the ionic environment inside the cell differs markedly from that outside, and thus, for the study of intracellular enzymes, physiological salt solutions are unphysiological. If better results are obtained by the addition of such solutions to broken cell suspensions, it may simply mean that the salts are acting on the residual permeabilities of partially damaged cells rather than on the enzymes directly.

The physiological significance of results obtained in vitro must be obtained by physiological means, i.e., by experiments on the whole animal. The classical method for studying intermediary metabolism in which substance $x$ is fed and substance $y$ is isolated in the urine does not ordinarily give enough detailed information about the fate of the substance. Correlation with the data obtained with isolated tissue suspensions is therefore often impossible. With the increasing use of labeled atoms animal experiments are yielding more precise results and thus offer promise of better correlation between in vitro and in vivo results. The story of sarcosine illustrates this point. When it is fed to a rabbit with benzoic acid, an increased amount of hippuric acid is excreted. This shows that sarcosine in some way gives rise to glycine. When sarcosine with labeled nitrogen is fed, the hippuric acid excreted contains the labeled nitrogen. This shows that sarcosine must be demethylated in the body but not deaminated. When sarcosine is added to liver suspensions an extra oxygen uptake occurs and glycine is formed. The sarcosine undergoes an oxidative demethylation. In this series of experiments the in vitro results serve to elucidate the mechanism of a reaction known to occur in the intact animal. Only in this way is the physiological significance of results with tissue experiments established.

Workers in tissue respiration tend to look for reactions of general significance. Results obtained with yeast or hashed pigeon breast muscle are applied with great facility to mammalian tissue. The omnipresence of the cytochrome oxidase and succinoxidase encourages this attitude, but does not justify it. Animals that differ so profoundly, physiologically, pharmacologically, and in all other ways, undoubtedly show differences in their respiratory mechanisms. Perhaps in the future more information will be obtained if these differences between animals are emphasized rather than minimized in an attempt to provide universal mechanisms that may have only superficial similarity. 


\section{PATHWAYS OF CARBOHYDRATE METABOLISM}

\section{E. S. GUZMAN BARRON \\ University of Chicago}

Whether the metabolism of carbohydrates by animal tissues is always accomplished according to Embden-Meyerhof's and Cori's schemes of phosphorylations and oxidation-reductions has not yet been established. In fact, if the normal pathway is obstructed, the breakdown of carbohydrates might proceed through other pathways. Direct oxidation of glucose, of hexosemonophosphate, and of phosphoglycerate might be the accessory pathways, although none of these has yet been shown to occur in animal tissues. The oxidation of a single substance may proceed via different enzyme systems; thus in sea urchin eggs (with no succinodehydrogenase and no cytochromes) the metabolism of carbohydrates undoubtedly proceeds through different pathways than in sperm (with succinodehydrogenase and cytochromes). The interesting findings of Korr (oxidations not inhibited by azide in resting cells and inhibited by azide when the cells are in active work) can be presented as examples in favor of this opinion.

The existence of these multiple pathways makes possible the orientation of reactions which occur continually in living cells, the metabolism of pyruvate being the clearest example. This is illustrated in the scheme shown on page 279 in which only the pertinent steps are reproduced.

Pyruvate is an extremely reactive substance, and seventeen different pathways of its metabolism are known to exist in living cells. In animal tissues, in the absence of oxygen, part of the pyruvate formed during the breakdown of carbohydrate is reduced by dihydrodiphosphopyridine nucleotide $\left(\mathrm{Py}\left(\mathrm{PO}_{4}\right)_{2} \mathrm{H}_{2}\right)$ to lactate, a reaction the extent of which represents the degree of anoxia; part of it may be reduced to alanine (Warburg and Christian's $d$-amino acid oxidase) or may be used for transaminations or dismutations. In the presence of oxygen, pyruvate activated by diphosphothiamineprotein may be oxidized to acetylphosphate; may, through condensation reactions, be responsible for the synthesis of alpha-ketoglutarate, cisaconitate, acetoacetate, acetylmethylcarbinol, or carbohydrate. It might, as postulated by Wood and Werkman, combine with carbon dioxide to give oxalacetate, an important reaction still eluding direct demonstration, which is the base of many hypotheses for the breakdown and synthesis of carbohydrate. Oxalacetate formed in this way may be reduced by $\mathrm{Py}\left(\mathrm{PO}_{4}\right)_{2} \mathrm{H}_{2}$ to malate, thus 
starting the oxidative pathway through Szent-Györgyi's cycle, or it may produce phosphopyruvic acid and thus start the synthesis of carbohydrate. These manifold reactions of pyruvate, all of them present in different degrees in animal tissues, show that the orientation of reactions during the metabolism of carbohydrates is extremely complex, and certainly different from tissue to tissue.

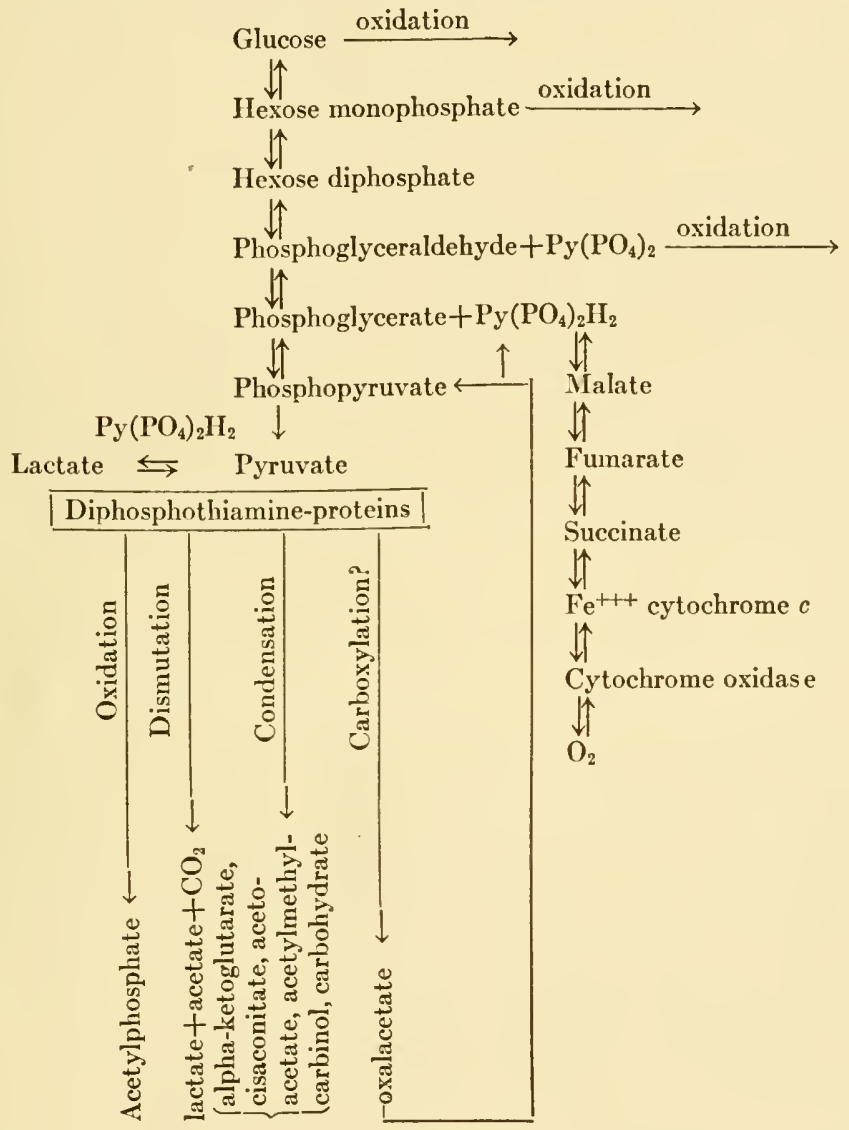




\section{THE CITRIC ACID CYCLE IN TISSUE METABOLISM}

FREDRICK J. STARE

Washington University School of Medicine, St. Louis

This is an appropriate time to mention the reasons why some of us do not believe in the importance, or even the presence, of citric acid as a component of the metabolic cycle generally termed the "citric acid cycle." The citric acid cycle is based on carefully established experimental facts. Some of these were mentioned this morning by Dr. Evans. We question, however, the application of these facts, particularly the presence of citrate, to a cycle of importance in respiring muscle.

It was only with high concentrations of pyruvate that Krebs was able to demonstrate increases in citrate, and these increases were of a small order, varying from 1 to 15 per cent. In tissue and body fluids, pyruvate occurs in a far lower concentration.

It was only with high concentrations of pyruvate, citrate, and malonate that an increase in succinate was detected. I know of no evidence, but I believe that a high concentration of glutamate, and other related compounds not included in the citric acid cycle, would also yield increases in succinate in the presence of a high malonate concentration.

The citric acid cycle assumes that malonate completely prevents the anaerobic reduction of oxalacetate to succinate, but there is no adequate proof for this assumption.

Citrate is definitely a weaker substance, as compared with the other members of the cycle, in increasing or prolonging the oxygen consumption of respiring muscle or in effecting pyruvate removal by respiring muscle. In fact, citrate frequently inhibits such effects.

Malonate in a concentration of $0.005 \mathrm{M}$ which is 5 to 6 times less than the concentration used by Krebs, inhibits oxygen uptake and pyruvate utilization on an average of 70 to 75 per cent (pigeon breast muscle). It completely and always inhibits any catalytic effect that citric acid may show. Yet any other member of the cycle when added in an equal concentration of $0.005 \mathrm{M}$ will completely overcome the malonate inhibition of oxygen uptake and of pyruvate removal. If the latter two depend in any way on a mechanism involving citric acid, the malonate should stop it because citric acid activity is always inhibited by malonate.

Recently, in Dr. Barron's laboratory, Lipton, Goldinger, and I have studied pyruvate and citrate utilization in respiring pigeon muscle tissue. According to the citric acid cycle, each molecule of 
pyruvate is converted to citrate in the course of its oxidation. The cycle implies that either citrate should be oxidized as rapidly as pyruvate, or that if citrate is not oxidized as rapidly as pyruvate, but still is a stage in the removal of pyruvate, it should accumulate in quantities sufficient to account for the difference in the rates of utilization of the two compounds. We found that pyruvate is oxidized at a far greater rate than citrate, and that citrate does not accumulate.

Our experimental observations favor a cycle involving a conversion of pyruvic acid to alpha-ketoglutaric acid, without citrate as an intermediary, followed by the Szent-Györgyi series of conversions of the dicarboxylic acids to oxalacetate. The occasional catalysis of respiration observed when citrate is added to muscle is probably not due to citrate itself but rather to alpha-ketoglutarate and the four-carbon acids which may be formed from it. Citrate may serve as a "stockroom" for the essential catalysts, exerting an effect on respiration only when they are low. Its synthesis from pyruvate may represent an unusual side reaction which under normal conditions is of little significance. However, under specific experimental conditions in vitro, such as large amounts of pyruvate, the rate of their reaction may be accelerated. A similar condition, with a high level of pyruvate, appears to exist in vivo. Thus Sober, Lipton, and Elvehjem found that in the recovery from acute thiamine deficiency large amounts of citrate are excreted.

In concluding these remarks, may I emphasize that these criticisms of the citric acid cycle are directed against a citric acid cycle which contains citric acid; they do not apply to a citric acid cycle which contains no citric acid. Proponents of the citric acid cycle quite properly spend most of their energy proving that some sort of a cycle exists rather than attempting to answer the question whether citric acid is, or is not, a member of it. 






